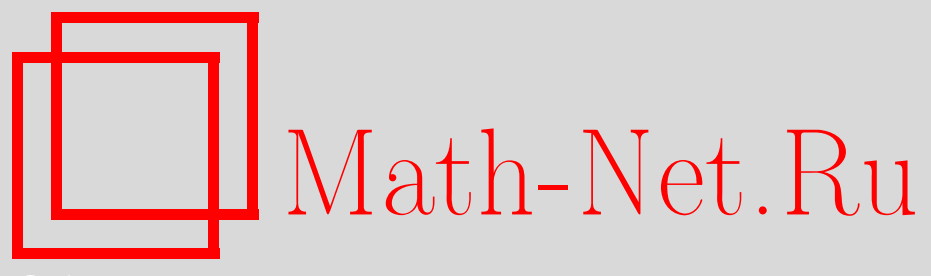

В. М. Бухштабер, Н. Ю. Ероховец, Многогранники, числа Фибоначчи, алгебры Хопфа и квазисимметрические функции, УМН, 2011, том 66, выпуск 2, 67-162

DOI: https://doi.org/10.4213/rm9421

Использование Общероссийского математического портала Math-Net.Ru подразумевает, что вы прочитали и согласны с пользовательским соглашением http://www.mathnet.ru/rus/agreement

Параметры загрузки:

IP : 18.234 .197 .8

26 апреля 2023 г., 18:24:25

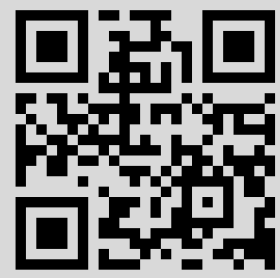




\title{
Многогранники, числа Фибоначчи, алгебры Хопфа и квазисимметрические функции
}

\author{
В. М. Бухштабер, Н. Ю. Ероховец
}

Обзор посвящен классической проблеме флаговых чисел выпуклых многогранников. Излагаются результаты, полученные на основе связи теории выпуклых многогранников с рядом современных направлений исследований.

Библиография: 62 названия.

Ключевые слова: флаговые числа, флаговые полиномы, алгебра Лейбница-Хопфа, слова Линдона, соотношения Дена-Соммервилля, универсальный $G$-полином, $\boldsymbol{c d}$-индекс.

\section{СОДЕРЖАНИЕ}

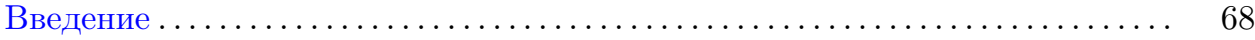

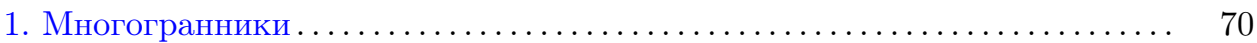

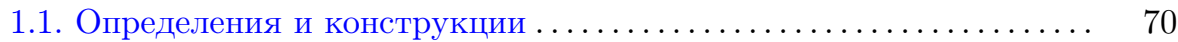

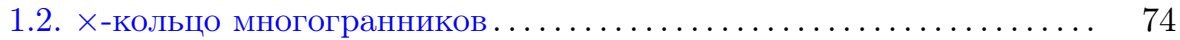

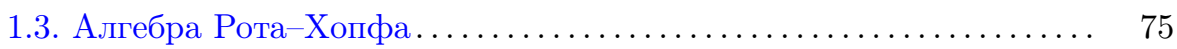

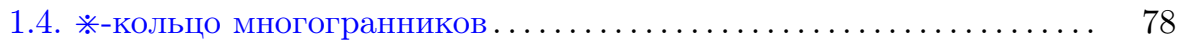

1.5. Операторы на кольцах многогранников ................. 80

1.6. Флаговые векторы . . . . . . . . . . . . . . . . . . . . 88

2. Квазисимметрические функции и алгебры Хопфа................ 93

2.1. Определения и конструкции ........................ 94

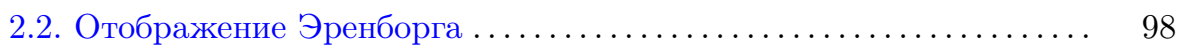

2.3. Алгебры Лейбница-Хопфа............................. 98

2.4. Алгебры Ли-Хопфа.................................. 102

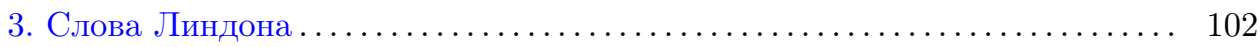

4. Топологическая реализация алгебр Хопфа...................... 106

5. Структура алгебры операторов граней $\mathcal{D} \ldots \ldots \ldots \ldots \ldots \ldots \ldots \ldots \ldots \ldots$

Работа выполнена при поддержке РФФИ (гранты 10-01-92102-ЯФ-а, 11-01-00694-а) и гранта Президента РФ МД-2253.2011.1.

(C) В. М. Бухштабер, Н. Ю. Ероховец, 2011 


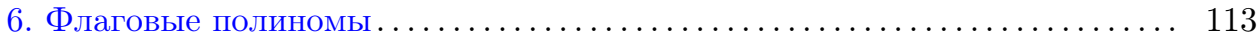

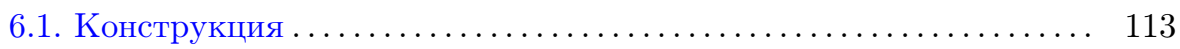

6.2. Кольца флаговых векторов . . . . . . . . . . . . . . . . . . . . . . 114

6.3. Характеризация флагового полинома $\mathcal{F}_{\mathcal{P}} \ldots \ldots \ldots \ldots \ldots \ldots \ldots \ldots$

6.4. Операторы бипирамиды и конуса....................... 122

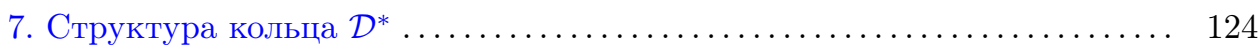

7.1. Основная теорема.............................. 124

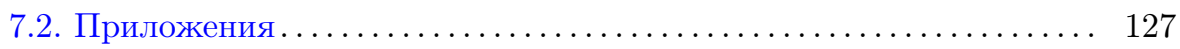

8. Мультипликативная структура колец флаговых векторов ........... 130

8.1. Основная теорема ........................... 130

8.2. Мультипликативные образующие ..................... 132

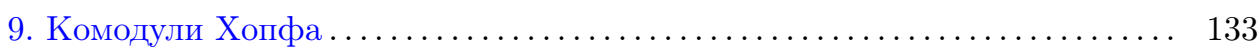

9.1. Модули и комодули Хопфа............................. 134

9.2. Каноническая структура на $×$-кольце многогранников . . . . . . 135

9.3. Комодульные структуры и гомоморфизмы ............. 136

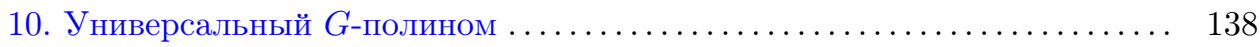

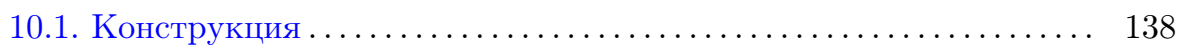

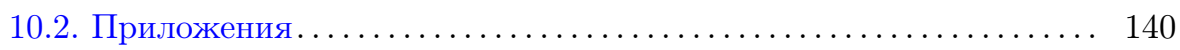

10.3. $g$ - и $h$-полиномы выпуклых многогранников ............... 142

10.4. Комментарии................................... 144

11. Гомоморфизмы колец выпуклых многогранников .............. 145

12. Проблема флаговых векторов . . . . . . . . . . . . . . . . . . . 146

Приложение А. Полиномиальность колец многогранников ............. 146

Приложение В. Кольцо флаговых чисел........................ 147

Приложение С. Алгебра Стембриджа ......................... 149

Приложение D. Операторы Пьери ........................... 149

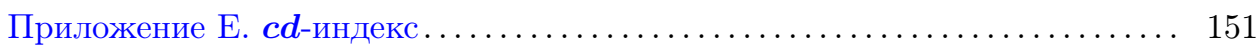

Е.1. Определения и конструкции ....................... 151

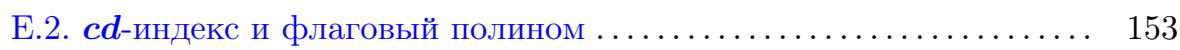

Е.3. Геометрический смысл $\boldsymbol{c d}$-индекса . . ................. 154

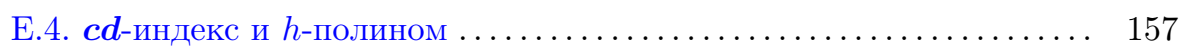

Е.5. Комментарии .................................. 159

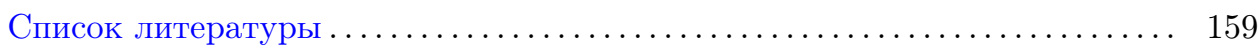

\section{Введение}

Многогранники - центральный объект выпуклой геометрии. В последнее время в этой классической области исследований используются результаты алгебраической геометрии и топологии, коммутативной и гомологической алгебры. Имеются замечательные результаты, лежащие на пересечении теории многогранников, теории комплексных многообразий, эквивариантной топологии и теории особенностей (см. [1]). Примеры взаимодействия теорий многогранников и дифференциальных уравнений можно найти в [2]. 
Настоящий обзор посвящен проблеме флаговых чисел выпуклых многогранников. Рассматривается градуированная группа, порожденная классами комбинаторной эквивалентности выпуклых многогранников вместе с умножениями, задаваемыми прямым произведением и джойном выпуклых многогранников. Таким образом, вводятся $\times$ - и $*$-кольца многогранников $\mathcal{P}$ и $\mathcal{R} \mathcal{P}$. Показано, что $*$-кольцо $\mathcal{R} \mathcal{P}$ имеет каноническую структуру градуированной алгебры Хопфа (алгебра Хопфа решеток частично упорядоченных по вложению граней многогранников). Более того, $\times$-кольцо $\mathcal{P}$ является комодулем Хопфа над $\mathcal{R} \mathcal{P}$. Описаны результаты применения теорий алгебр Хопфа, дифференциальных градуированных алгебр и квазисимметрических функций к построению комбинаторных инвариантов многогранников. В качестве следствия мы получаем инварианты, различающие многогранники с одинаковыми флаговыми числами.

В теории многогранников важную роль играют соотношения Дена-Соммервилля для векторов граней простых многогранников и Байер-Биллера для флаговых векторов выпуклых многогранников. Используя вектор граней, мы строим $f$-полином многогранника и показываем, что условия Дена-Соммервилля эквивалентны функциональным уравнениям, выделяющим образ кольща nростых многогранников в кольце $\mathbb{Z}[\alpha, t]$. Обобщением этой конструкции является гомоморфизм $\mathcal{F}_{\mathcal{P}}: \mathcal{P} \rightarrow \mathcal{Q} s y m[\alpha]$, где $\mathcal{Q} s y m$ - алгебра Хопфа квазисимметрических функций, изоморфная кольцу полиномов над $\mathbb{Z}$. Мы описываем функциональные уравнения, выделяющие образ кольца $\mathcal{P}$ в $\mathcal{Q} \operatorname{sym}[\alpha]$ в виде аддитивного прямого слагаемого. Гомоморфизм $\mathcal{F}_{\mathcal{P}}$ записывается в терминах флаговых чисел, и мы показываем, что наши уравнения эквивалентны соотношениям Байер-Биллера на эти числа.

Мы вводим правые представления алгебры $\mathbb{Z}\langle\boldsymbol{c}, \boldsymbol{d}\rangle$ на кольцах $\mathcal{P}$ и $\mathcal{Q} \operatorname{sym}[\alpha]$ такие, что гомоморфизм $\mathcal{F}_{\mathcal{P}}$ является эквивариантным и его образ совпадает с орбитой $1 \cdot \mathbb{Z}\langle\boldsymbol{c}, \boldsymbol{d}\rangle$. Оказывается, что для выпуклого многогранника $P$ разложение квазисимметрической функции $\mathcal{F}_{\mathcal{P}}(P)$ по базису из $\boldsymbol{c d}$-слов совпадает с известным $\boldsymbol{c d}$-индексом. Более того, мы получаем разложение $\boldsymbol{c d}$-базиса по базису квазисимметрических мономов. Важным результатом является то, что все коэффициенты в указанных выражениях неотрицательны.

Согласно теореме Байер-Биллера, размерность линейного пространства над полем рациональных чисел $\mathbb{Q}$, натянутого на флаговые векторы выпуклых $n$-мерных многогранников, равна $n$-му числу Фибоначчи. Используя этот результат, мы показываем, что образ кольца $\mathcal{P} \otimes \mathbb{Q}$ в $\mathcal{Q} \operatorname{sym}[\alpha] \otimes \mathbb{Q}$ является кольцом полиномов. Таким образом, мы получаем разложение производящей функции чисел Фибоначчи в бесконечное произведение и, в качестве следствия, формулу для числа мультипликативных образующих этого кольца полиномов. Теория алгебр Хопфа дает условия, выделяющие его мультипликативные образующие, а теория квазисимметрических функций позволяет описать эти образующие в терминах специальных слов Линдона. Мы даем конструкцию универсального $G$-полинома, соответствующего деформации умножения в градуированном кольце, и показываем, что фундаментальные понятия теории выпуклых многогранников (торические $g$ - и $h$-полиномы) являются частными случаями общеалгебраических результатов.

В заключительной части обзора мы приводим замечательную геометрическую конструкцию К. Ли, следствием которой являются выражения $\boldsymbol{c d}$-индек- 
са и $h$-полинома многогранника в виде суммы по вершинам. Слагаемые в этой сумме рекуррентно описываются при помощи функции высоты многогранника.

Ссылки на работы, содержащие ключевые результаты, о которых идет речь во введении, даны в основном тексте. В целом обзор опирается на работы авторов [2]-[5].

\section{1. Многогранники}

1.1. Определения и конструкции. В этом пункте собраны необходимые определения и конструкции из теории многогранников. Подробности можно найти в книгах [6]-[9].

Существует два алгоритмически различных способа задать выпуклый многогранник в линейном пространстве. Далее мы будем рассматривать многогранники только в евклидовом пространстве $\mathbb{R}^{n}, n<\infty$.

ОПРЕДЕЛЕНИЕ 1.1. $\mathcal{V}$-многогранником называется выпуклая оболочка конечного набора точек в $\mathbb{R}^{n}$.

ОПРЕДЕЛЕНИЕ 1.2. $\mathcal{H}$-полиэдром называется пересечение конечного набора замкнутых полупространств в $\mathbb{R}^{n}$.

$\mathcal{H}$-многогранником называется ограниченный $\mathcal{H}$-полиэдр.

Согласно классической теореме (см., например, [7; теорема 1.1]), эти два определения описывают один и тот же объект.

Размерность выпуклого многогранника определяется как размерность его аффинной оболочки. Если специально не оговорено, то без ограничения общности мы будем предполагать, что $n$-мерный выпуклый многогранник $P^{n}$ лежит в пространстве $\mathbb{R}^{n}$, нулевая точка которого является внутренней для этого многогранника. В этом случае существуют такие векторы $\boldsymbol{a}_{1}, \ldots, \boldsymbol{a}_{m}$, что

$$
P^{n}=\left\{\boldsymbol{x} \in \mathbb{R}^{n}:\left\langle\boldsymbol{a}_{i}, \boldsymbol{x}\right\rangle+1 \geqslant 0, i=1, \ldots, m\right\}
$$

- многогранник с гипергранями $F_{i}=P^{n} \cap\left\{\boldsymbol{x} \in \mathbb{R}^{n}:\left\langle\boldsymbol{a}_{i}, \boldsymbol{x}\right\rangle+1=0\right\}, i=1, \ldots, m$.

Каждому многограннику $P$ соответствует решетка граней $L(P)$ с частичным порядком, задаваемым вложением граней. Эта решетка имеет минимальный элемент (пустое множество) и максимальный элемент (сам многогранник).

Два многогранника $P$ и $Q$ называются комбинаторно эквивалентными, если существует взаимно однозначное соответствие между $L(P)$ и $L(Q)$, coxpaняющее отношение включения. Комбинаторным многогранником называется класс комбинаторной эквивалентности выпуклых многогранников. Во многих случаях для описания свойств комбинаторных многогранников удобно использовать их геометрических представителей вида (1). Ниже мы приводим конструкции для геометрических многогранников, но нетрудно видеть, что эти конструкции корректно определены на классах комбинаторной эквивалентности.

Понятие многогранника общего положения с точки зрения определений 1.1 и 1.2 приводит к двум специальным классам многогранников.

ОПРЕДЕЛЕНИЕ $1.3\left(\mathcal{V}\right.$-многогранник общего положения). Многогранник $P^{n}$ называется симплициальным, если каждая его гипергрань является симплексом (т. е. содержит ровно $n$-вершин). 
ОПРЕДЕлЕНИЕ 1.4 ( $\mathcal{H}$-многогранник общего положения). Многогранник $P^{n}$ называется простым, если каждая его вершина содержится ровно в $n$ гипергранях.

Нам потребуется также следующее понятие.

ОПРЕДЕЛЕНИЕ 1.5 (двойственный многогранник). Для $n$-мерного выпуклого многогранника $P^{n}$, представленного в форме (1), определен многогранник

$$
\left(P^{n}\right)^{*}=\left\{\boldsymbol{y} \in \mathbb{R}^{n}:\langle\boldsymbol{y}, \boldsymbol{x}\rangle+1 \geqslant 0 \forall \boldsymbol{x} \in P^{n}\right\},
$$

который называется двойственным или полярным.

Используя то, что начало координат является внутренней точкой многогранника $P^{n}$, представленного в форме (1), можно показать (см., например, [7]), что

$$
\left(P^{n}\right)^{*}=\operatorname{conv}\left\{\boldsymbol{a}_{i}, i=1, \ldots, m\right\} \quad \text { и } \quad\left(P^{*}\right)^{*}=P .
$$

Существует взаимно однозначное соответствие $F \leftrightarrow F^{\diamond}$ между $i$-мерными гранями $n$-мерного многогранника $P$ и $(n-i-1)$-мерными гранями многогранника $P^{*}$ такое, что

$$
F \subset G \quad \Leftrightarrow \quad G^{\diamond} \subset F^{\diamond}
$$

Для любого простого многогранника $P$ двойственный многогранник $P^{*}$ является симплициальным, и наоборот.

ОПРЕДЕЛЕНИЕ 1.6 (гранная фигура). Пусть $F$ - грань многогранника $P$. Гранной фигурой $P / F$ называется многогранник

$$
P / F=\left(F^{\diamond}\right)^{*} .
$$

Легко видеть, что размерность многогранника $P / F$ равна $(\operatorname{dim} P-\operatorname{dim} F-1)$, а его решетка граней имеет вид

$$
L(P / F)=\{G \in L(P): F \subseteq G \subseteq P\},
$$

т. е. $L(P / F)$ является отрезком $[F, P]$ в решетке $L(P)$.

ПримеР 1.7. Пусть $P^{n}$ - простой многогранник. Тогда для любой собственной $k$-мерной грани $F \subset P$ гранная фигура $P / F$ является $(n-k-1)$-мерным симплексом.

ОПРЕДЕЛЕНИЕ 1.8 (прямое произведение). Для выпуклых многогранников $P^{n_{1}} \subset \mathbb{R}^{n_{1}}$ и $Q^{n_{2}} \subset \mathbb{R}^{n_{2}}$ определен выпуклый многогранник

$$
P^{n_{1}} \times Q^{n_{2}}=\left\{(\boldsymbol{x}, \boldsymbol{y}) \subset \mathbb{R}^{n_{1}} \times \mathbb{R}^{n_{2}}: \boldsymbol{x} \in P^{n_{1}}, \boldsymbol{y} \in Q^{n_{2}}\right\} .
$$

Эта операция называется прямым произведением выпуклых многогранников. Она является коммутативной и ассоциативной.

Многогранник $P^{n_{1}} \times Q^{n_{2}}$ имеет размерность $n_{1}+n_{2}$, а его грани являются прямыми произведениями граней многогранников $P^{n_{1}}$ и $Q^{n_{2}}$. В частности, он имеет $m_{1}+m_{2}$ гиперграней, где $m_{1}$ и $m_{2}$ - числа гиперграней многогранников $P^{n_{1}}$ и $Q^{n_{2}}$ соответственно. 
ОПРЕДЕЛЕНИЕ 1.9 (скрещенное произведение). Для выпуклых многогранников

$$
\begin{aligned}
& P^{n_{1}}=\left\{\boldsymbol{x} \in \mathbb{R}^{n_{1}}:\left\langle\boldsymbol{a}_{i}, \boldsymbol{x}\right\rangle+1 \geqslant 0, i=1, \ldots, m_{1}\right\}, \\
& Q^{n_{2}}=\left\{\boldsymbol{y} \in \mathbb{R}^{n_{2}}:\left\langle\boldsymbol{b}_{j}, \boldsymbol{y}\right\rangle+1 \geqslant 0, j=1, \ldots, m_{2}\right\}
\end{aligned}
$$

определен многогранник

$$
P^{n_{1}} \circ Q^{n_{2}}=\operatorname{conv}\left(P^{n_{1}} \times\{\mathbf{0}\} \cup\{\mathbf{0}\} \times Q^{n_{2}}\right) \subset \mathbb{R}^{n_{1}} \times \mathbb{R}^{n_{2}} .
$$

Эта операция называется скрещенным произведением выпуклых многогранников. Она является коммутативной и ассоциативной. Многогранник $P^{n_{1}} \circ Q^{n_{2}}$ имеет размерность $n_{1}+n_{2}$.

УТВЕРЖДЕНИЕ 1.10. Прямое и скрещенное произведения многогранников связаны формулой

$$
\left(P^{n_{1}} \times Q^{n_{2}}\right)^{*}=\left(P^{n_{1}}\right)^{*} \circ\left(Q^{n_{2}}\right)^{*} .
$$

ДокАЗАтельство. Рассмотрим представление многогранника $P^{n_{1}} \times Q^{n_{2}}$ в форме (1):

$$
\begin{aligned}
& P^{n_{1}} \times Q^{n_{2}}=\left\{(\boldsymbol{x}, \boldsymbol{y}) \in \mathbb{R}^{n_{1}} \times \mathbb{R}^{n_{2}}:\left\langle\boldsymbol{a}_{i}, \boldsymbol{x}\right\rangle+1 \geqslant 0, i=1, \ldots, m_{1},\right. \\
& \left.\left\langle\boldsymbol{b}_{j}, \boldsymbol{y}\right\rangle+1 \geqslant 0, j=1, \ldots, m_{2}\right\} \text {. }
\end{aligned}
$$

Согласно формуле (2), мы получаем:

$$
\begin{aligned}
\left(P^{n_{1}} \times Q^{n_{2}}\right)^{*} & =\operatorname{conv}\left\{\left(\boldsymbol{a}_{i}, \mathbf{0}\right),\left(\mathbf{0}, \boldsymbol{b}_{j}\right), i=1, \ldots, m_{1}, j=1, \ldots, m_{2}\right\} \\
& =\left(P^{n_{1}}\right)^{*} \circ\left(Q^{n_{2}}\right)^{*},
\end{aligned}
$$

что и доказывает утверждение.

Прямое произведение двух простых многогранников является простым многогранником. Следовательно, для любых двух симплициальных многогранников $P$ и $Q$ скрещенное произведение $P \circ Q$ является симплициальным многогранником.

Стандартным $n$-мерным кубом $I^{n}$ называется многогранник $I^{n}=\left\{\boldsymbol{x} \in \mathbb{R}^{n}\right.$ : $\left.0 \leqslant x_{i} \leqslant 1, i=1, \ldots, n\right\}$. Положим $I=I^{1}$.

ПРимеР 1.11 (бипирамида). Бипирамидой (или надстройкой) многогранника $P$ называется многогранник $B P=I \circ P$.

ПримеР 1.12 (призма). Призмой (или иилиндром) многогранника $P$ называется многогранник $I \times P$. Согласно формуле $(3)$, мы получаем $(B P)^{*}=I \times P^{*}$.

Правильным симплексом $\Delta^{n-1} \subset \mathbb{R}^{n}$ называется выпуклая оболочка стандартных базисных векторов в $\mathbb{R}^{n}$.

ОПРЕДЕЛЕНИЕ 1.13 (джойн). Пусть $P^{n_{1}} \subset \mathbb{R}^{n_{1}+1}$ и $Q^{n_{2}} \subset \mathbb{R}^{n_{2}+1}-$ два многогранника, лежащих в правильных симплексах $\Delta^{n_{1}}$ и $\Delta^{n_{2}}$. Многогранник

$$
P^{n_{1}} * Q^{n_{2}}=\operatorname{conv}\left(P^{n_{1}} \times\{\mathbf{0}\} \cup\{\mathbf{0}\} \times Q^{n_{2}}\right) \subset \mathbb{R}^{n_{1}+1} \times \mathbb{R}^{n_{2}+1}
$$

называется джойном многогранников $P^{n_{1}}$ и $Q^{n_{2}}$. Многогранник $P^{n_{1}} * Q^{n_{2}}$ имеет размерность $\left(n_{1}+n_{2}+1\right)$ и лежит в правильном симплексе $\Delta^{n_{1}+n_{2}+1}$.

Из конструкции видно, что операция джойна является коммутативной и ассоциативной. 
ПримеР 1.14 (конус). Конусом (или пирамидой) многогранника $P$ называется многогранник $C P=\mathrm{pt} * P$, где $\mathrm{pt}-$ точка.

Отметим, что любая вершина $v$ многогранника $P$ задает вложение конуса $C Q=\mathrm{pt} * Q$ в многогранник $P * Q$, при котором точка pt переходит в вершину $v$.

Термины “призма", “пирамида" и "бипирамида" используются в комбинаторике многогранников. Они соответствуют терминам "цилиндр", "конус" и "надстройка" в комбинаторной топологии.

В определении операции джойна мы рассматривали многогранники, лежащие в правильных симплексах. Однако аффинный тип многогранника $P * Q$ не зависит от расположения многогранников $P$ и $Q$ в правильных симплексах. Он зависит только от аффинных типов многогранников $P$ и $Q$.

Многогранник $P * Q$ может быть определен и в инвариантных терминах как выпуклая оболочка многогранников $P$ и $Q$, при условии, что они расположены в некотором пространстве $\mathbb{R}^{N}$ таким образом, что их аффинные оболочки aff $P$ и aff $Q$ скрещиваются по $\{0\}$ в смысле линейной алгебры.

Пустое множество $\varnothing$ удобно рассматривать как $(-1)$-мерный многогранник. В этом случае в дополнение к формуле (4) можно написать:

$$
\varnothing * P=P=P * \varnothing \quad \text { и } \varnothing * \varnothing=\varnothing .
$$

В тех случаях, когда рассматриваются операции джойна и скрещенного произведения, удобно считать пустое множество гранью многогранника, в то же время при операции прямого произведения естественно рассматривать только непустые грани.

УТВЕРЖДЕНИЕ 1.15. Грани многогранника $P^{n_{1}} * Q^{n_{2}}$ в конструкиии (4) c точностъю до аффинной эквивалентности имеют вид $F * G$, где $F, G$ грани многогранников $P$ и $Q$ соответственно.

Внутри многогранника $P * Q$ лежат многогранники $P$ и $Q$, которые удобно отождествлять с $P * \varnothing$ и $\varnothing * Q$.

СледСтвиЕ 1.16. Решетка граней $L(P * Q)$ является прямым произведением решеток $L(P)$ и $L(Q)$. Таким образом, операчия "джойн" корректно определена на комбинаторных многогранниках.

ПримеР 1.17. Для симплексов $\Delta^{k}$ и $\Delta^{l}$ мы имеем: $\Delta^{k} * \Delta^{l}=\Delta^{k+l+1}$.

УТВЕРЖДЕНИЕ 1.18. Грани многогранника $P^{n_{1}} \circ Q^{n_{2}}$ с точностъю до аффинной эквивалентности имеют вид $P \circ Q$ или $F * G$, где $F \neq P, G \neq Q$ грани многогранников $P$ и $Q$ соответственно.

УТВЕРЖДЕНИЕ 1.19. Пусть $P$ и - комбинаторные многогранники. Тогда

$$
(P * Q)^{*}=P^{*} * Q^{*} \text {. }
$$

ДоказАТЕЛЬСтво. Грани многогранника $(P * Q)^{*}$ имеют вид $(F * G)^{\diamond}$, причем $(F * G)^{\diamond} \subseteq\left(F^{\prime} * G^{\prime}\right)^{\diamond}$ тогда и только тогда, когда $F^{\prime} * G^{\prime} \subseteq F * G$, т. е. $F^{\prime} \subseteq F, G^{\prime} \subseteq G$, что равносильно условию $F^{\diamond} \subseteq\left(F^{\prime}\right)^{\diamond}, G^{\diamond} \subseteq\left(G^{\prime}\right)^{\diamond}$. Поэтому соответствие $(F * G)^{\diamond} \leftrightarrow F^{\diamond} * G^{\diamond}$ задает комбинаторную эквивалентность многогранников $(P * Q)^{*}$ и $P^{*} * Q^{*}$. 
СлеДСтвИЕ 1.20. Пусть $P$ - комбинаторный многогранник. Тогда $(C P)^{*}=$ $C P^{*}$, m.е. операщия взятия конуса и операщия перехода $\kappa$ двойственному многограннику коммутируют.

\section{2. ×-кольцо многогранников.}

ОПРЕдЕлЕниЕ 1.21. Обозначим через $\mathcal{P}^{2 n}$ свободную абелеву группу, порожденную всеми $n$-мерными комбинаторными многогранниками. В частности, $\mathcal{P}^{0}=\mathbb{Z}, \mathcal{P}^{2}=\mathbb{Z}$ с образующими pt - точка и $I$ - интервал соответственно. Для $n>1$ имеет место разложение в прямую сумму

$$
\mathcal{P}^{2 n}=\sum_{m \geqslant n+1} \mathcal{P}^{2 n, 2(m-n)},
$$

где группа $\mathcal{P}^{2 n, 2(m-n)}$ порождена $n$-мерными многогранниками с $m$ гипергранями. Нетрудно показать, что $\operatorname{rk} \mathcal{P}^{2 n, 2(m-n)}<\infty$ для любых $m$ и $n, n<m$. Прямое произведение многогранников $P \times Q$ задает в прямой сумме

$$
\mathcal{P}=\sum_{n \geqslant 0} \mathcal{P}^{2 n}=\mathcal{P}^{0} \oplus \mathcal{P}^{2} \oplus \sum_{m \geqslant 3} \sum_{n=2}^{m-1} \mathcal{P}^{2 n, 2(m-n)}
$$

структуру биградуированного коммутативного ассоциативного кольца, которое мы будем называть $\times$-кольцом многогранников. Роль единицы в этом кольце играет точка $P^{0}=\mathrm{pt}$.

Прямое произведение $P \times Q$ простых многогранников $P$ и $Q$ является простым многогранником, поэтому группа $\mathcal{P}_{\mathrm{s}}$, порожденная всеми простыми многогранниками, является подкольцом в $\mathcal{P}$.

ОПРЕДЕЛЕНИЕ 1.22. Комбинаторный многогранник $P^{n}, n>0$, называется неразложимым относительно прямого произведения, если он не может быть представлен как прямое произведение $P_{1} \times P_{2}$ двух многогранников положительной размерности.

УТВЕРЖДЕНИЕ 1.23. Колъцо $\mathcal{P}$ является кольцом полиномов от неразложимых относительно прямого произведения многогранников.

ДокАзАТЕЛЬСтво. См. приложение А (утверждение А.1).

Многогранник $P=P_{1} \times P_{2}$ является простым тогда и только тогда, когда оба многогранника $P_{1}$ и $P_{2}$ простые.

СЛЕДСТВИЕ 1.24. Кольцо $\mathcal{P}_{\mathrm{s}}$ является подкольцом полиномов от неразложимых простых многогранников.

Так как $\left(P^{*}\right)^{*}=P$, то соответствие $P \rightarrow P^{*}$ задает инволюцию кольца $\mathcal{P}$.

Для любого изоморфизма градуированных абелевых групп $A: \mathcal{P} \rightarrow \mathcal{P}$ определено умножение $P \times{ }_{A} Q=A^{-1}(A P \times A Q)$ такое, что отображение $A$ является изоморфизмом градуированных колец $\left(\mathcal{P}, \times_{A}\right) \rightarrow(\mathcal{P}, \times)$.

Инволюция $*: \mathcal{P} \rightarrow \mathcal{P}$ определяет умножение $P \times_{*} Q$. Используя формулу (2), мы получаем $P \times_{*} Q=P \circ Q$. 
ОПРЕДЕЛЕниЕ 1.25. Биградуировка кольца $\mathcal{P}$ задает кольцевой гомоморфизм

$$
\xi_{\alpha, \beta}: \mathcal{P} \rightarrow \mathbb{Z}[\alpha, \beta], \quad \xi_{\alpha, \beta}\left(P^{n}\right)=\alpha^{n} \beta^{m},
$$

где $m=m(P)$ - число гиперграней многогранника $P^{n}$ и $\operatorname{deg} \alpha=\operatorname{deg} \beta=2$.

ПРимеР 1.26. Далее важную роль будет играть кольцевой гомоморфизм $\xi_{\alpha}=\xi_{\alpha, 1}: \mathcal{P} \rightarrow \mathbb{Z}[\alpha]$. При $\alpha=0$, получаем аугментацию кольца $\mathcal{P}$

$$
\xi(P)= \begin{cases}1, & \text { если } P=\mathrm{pt}, \\ 0 & \text { в противном случае. }\end{cases}
$$

ОПРЕДЕЛЕНИЕ 1.27 [2]. $f$-полиномом выпуклого многогранника $P^{n}$ называется полином

$$
f(\alpha, t)\left(P^{n}\right)=\alpha^{n}+f_{n-1,1} \alpha^{n-1} t+\cdots+f_{1, n-1} \alpha t^{n-1}+f_{0, n} t^{n},
$$

где $f_{i, n-i}$ - число $i$-мерных граней многогранника $P^{n}$.

УТВЕРЖДЕНИЕ 1.28. $f$-полином выпуклых многогранников определяет кольцевой гомоморфизм $f: \mathcal{P} \rightarrow \mathbb{Z}[\alpha, t]$.

ДокАзАТЕЛьство. $f$-полином можно переписать в виде

$$
f(\alpha, t)\left(P^{n}\right)=\sum_{F \subseteq P} \alpha^{\operatorname{dim} F} t^{n-\operatorname{dim} F},
$$

где $F$ пробегает непустые грани многогранника $P$.

Так как грани прямого произведения $P^{n_{1}} \times Q^{n_{2}}$ являются прямыми произведениями граней многогранников $P^{n_{1}}$ и $Q^{n_{2}}$, то

$$
\begin{aligned}
f(\alpha, t)\left(P^{n_{1}} \times Q^{n_{2}}\right) & =\sum_{F \times G \subseteq P \times Q} \alpha^{\operatorname{dim} F+\operatorname{dim} G} t^{n_{1}+n_{2}-\operatorname{dim} F-\operatorname{dim} G} \\
& =\sum_{F \subseteq P, G \subseteq Q}\left(\alpha^{\operatorname{dim} F} t^{n_{1}-\operatorname{dim} F}\right)\left(\alpha^{\operatorname{dim} G} t^{n_{2}-\operatorname{dim} G}\right) \\
& =\left(\sum_{F \subseteq P} \alpha^{\operatorname{dim} F} t^{n_{1}-\operatorname{dim} F}\right)\left(\sum_{G \subseteq Q} \alpha^{\operatorname{dim} G} t^{n_{2}-\operatorname{dim} G}\right) \\
& =f(\alpha, t)\left(P^{n_{1}}\right) \cdot f(\alpha, t)\left(Q^{n_{2}}\right) .
\end{aligned}
$$

\section{3. Алгебра Рота-Хопфа.}

ОПРЕДЕЛЕНИЕ 1.29. Пусть $R_{1}$ и $R_{2}$ - кольца. Обозначим через $\tau_{R_{1}, R_{2}}$ кольцевой гомоморфизм $R_{1} \otimes R_{2} \rightarrow R_{2} \otimes R_{1}$, переставляющий тензорные сомножители: $\tau_{R_{1}, R_{2}}(x \otimes y)=y \otimes x$.

Пусть $A=\sum A^{2 n}, n \geqslant 0,-$ связное градуированное кольцо, где $A^{2 n}-$ группа элементов градуировки $2 n$. Введем кольцо $\mathcal{L}(A)=\sum \mathcal{L}_{-2 k}(A), k \in \mathbb{Z}$, линейных операторов $A \rightarrow A$, умножение в котором задается композицией операторов, а группу $\mathcal{L}_{-2 k}(A)$ составляют операторы, которые переводят группу $A^{2 n}$ в группу $A^{2(n-k)}$ для каждого $n$. Имеем id $=1 \in \mathcal{L}_{0}(A)$. 
ОПРЕДЕЛЕНИЕ 1.30. Пусть $T$ - конечное частично упорядоченное множество с минимальным элементом $\hat{0}$ и максимальным элементом $\hat{1}$.

Говорят, что элемент $y \in T$ накрывает элемент $x \in T$, если $x<y$ и в множестве $T$ нет такого элемента $z$, что $x<z<y$.

Частично упорядоченное множество $T$ называется градуированным, если существует функция ранга $\rho: T \rightarrow \mathbb{Z}$ такая, что $\rho(\hat{0})=0$ и $\rho(y)=\rho(x)+1$, если $y$ накрывает $x$. Легко видеть, что если частично упорядоченное множество $T$ является градуированным, то функция $\rho$ определена однозначно.

Для двух элементов $x, y \in T, x \leqslant y$, положим $\rho(x, y)=\rho(y)-\rho(x)$. Положим также $\rho(T)=\rho(\hat{1})$ и $\operatorname{deg} T=2 \rho(T)$.

Для элементов $x, y \in T$ таких, что $x \leqslant y$, определена функиия Мёбиуса $\mu(x, y)$, задаваемая рекуррентной формулой

$$
\mu(x, y)= \begin{cases}-\sum_{x \leqslant z<y} \mu(x, z), & \text { если } x<y, \\ 1, & \text { если } x=y .\end{cases}
$$

Функция Мёбиуса однозначно определена условием: для любых $x, y \in T, x \leqslant y$, имеем $\sum_{x \leqslant z \leqslant y} \mu(x, z)=1$, если $x=y$, и $\sum_{x \leqslant z \leqslant y} \mu(x, z)=0$ в противном случае.

Частично упорядоченное множество $T$ называется эйлеровым, если функция Мёбиуса имеет вид $\mu(x, y)=(-1)^{\rho(x, y)}$.

УтВЕРЖДЕНИЕ 1.31. Пусть $P$ - многогранник. Тогда его решетка граней $L(P)$ является эйлеровым частично упорядоченным множеством.

ДоказАтельство. Пусть $F, G \in L(P), F \subset G$, - две грани многогранника $P$. Тогда из формулы Эйлера-Пуанкаре для гранной фигуры $G / F$ имеем:

$$
\sum_{F \subset F^{\prime} \subset G}(-1)^{\operatorname{dim} F^{\prime}-\operatorname{dim} F-1}=1+(-1)^{\operatorname{dim} G-\operatorname{dim} F},
$$

откуда следует, что функция $(-1)^{\operatorname{dim} G-\operatorname{dim} F}$ удовлетворяет рекуррентному соотношению для функции Мёбиуса.

Многие из результатов этого обзора верны для случая эйлеровых частично упорядоченных множеств, но мы ограничимся рассмотрением выпуклых многогранников.

Частично упорядоченные множества $T^{\prime}$ и $T^{\prime \prime}$ называются изоморфными, если существует взаимно однозначное соответствие $T^{\prime} \rightarrow T^{\prime \prime}$, сохраняющее порядок.

Обозначим через $\mathcal{R}$ градуированную свободную абелеву группу, порожденную классами изоморфизма конечных градуированных частично упорядоченных множеств $T, \operatorname{deg} T=2 \rho(T)$.

УтВеРЖДЕНИЕ 1.32 [10], [11]. Группа $\mathcal{R}$ имеет структуру связной градуированной алгебры Хопфа:

- умножение $\mu: \mathcal{R} \otimes \mathcal{R} \rightarrow \mathcal{R}$ задается прямым (декартовым) произведением $T^{\prime} \times T^{\prime \prime}$ частично упорядоченных множеств $T^{\prime}$ u $T^{\prime \prime}$, в котором порядок определяется условием

$$
(x, y) \leqslant_{T^{\prime} \times T^{\prime \prime}}(u, v) \text { тогда и толъко тогда, когда } x \leqslant_{T^{\prime}} u \text { u } y \leqslant_{T^{\prime \prime}} v ;
$$


- единица $: \mathbb{Z} \rightarrow \mathcal{R}$ переводит единичу 1 кольи, $\mathbb{Z}$ в частично упорядоченное множество, состоящее из одного элемента $\hat{0}=\hat{1}$;

- коумножение $\Delta: \mathcal{R} \rightarrow \mathcal{R} \otimes \mathcal{R}$ задается формулой

$$
\Delta(T)=\sum_{\hat{0} \leqslant z \leqslant \hat{1}}[\hat{0}, z] \otimes[z, \hat{1}],
$$

где $[x, y]$ - частично упорядоченное подмножество $\{z \in T \mid x \leqslant z \leqslant y\}$;

- коединица $\varepsilon: \mathcal{R} \rightarrow \mathbb{Z}$ задается формулой

$$
\varepsilon(T)= \begin{cases}1, & \text { если } \hat{0}=\hat{1}, \\ 0 & \text { в противном случае }\end{cases}
$$

- антипод $\chi: \mathcal{R} \rightarrow \mathcal{R}$ действует по формуле

$$
\chi(T)=\sum_{k \geqslant 0} \sum_{C_{k}}(-1)^{k}\left[x_{0}, x_{1}\right] \cdot\left[x_{1}, x_{2}\right] \cdots\left[x_{k-1}, x_{k}\right]
$$

где $C_{k}=\left(\hat{0}=x_{0}<x_{1}<\cdots<x_{k}=\hat{1}\right)$.

ОПРедЕЛЕНИЕ 1.33. Алгебру Хопфа $(\mathcal{R}, \mu, \imath, \Delta, \varepsilon, \chi)$ мы будем называть $а л$ геброй Poma-Xonфа.

Алгебра Рота-Хопфа градуированных частично упорядоченных множеств появилась в [10]. Различные варианты этой конструкции исследовались в работах [11]-[14]. Обобщения этой алгебры можно найти в [15].

ПримеР 1.34. Простейшая булева алгебра $B_{1}=\{\hat{0}, \hat{1}\}$ является решеткой граней точки pt. В этом случае $\Delta\left(B_{1}\right)=1 \otimes B_{1}+B_{1} \otimes 1, \chi\left(B_{1}\right)=-B_{1}$.

В кольце $\mathcal{R}$ определена каноническая инволюция *. Образом частично упорядоченного множества $T$ при инволюции $*$ является градуированное частично упорядоченное множество $T^{*}$, состоящее из тех же элементов, но с обращенным порядком, т. е. $x \leqslant_{T} y$ тогда и только тогда, когда $x \geqslant_{T^{*}} y$. Тогда $\rho\left(T^{*}\right)=\rho(T)$ и $\Delta\left(T^{*}\right)=* \otimes *\left(\tau_{\mathcal{R}, \mathcal{R}} \Delta T\right)$, т. е. отображение $*$ является изоморфизмом алгебр

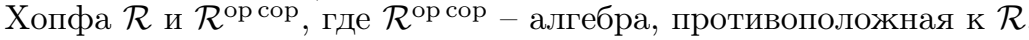

Введем операторы $\mathcal{R} \rightarrow \mathcal{R}$, которые нам потребуются в дальнейшем.

ОПРЕДЕЛЕНИЕ 1.35. Пусть $T \in \mathcal{R}^{2 n}$ - частично упорядоченное множество ранга $n, n \geqslant 1$. Введем частично упорядоченные множества $T_{1}=T \cup\left\{\hat{1}_{n+1}\right\}$, $T_{2}=\left(T \backslash\left\{\hat{1}_{n}\right\}\right) \cup\{x, y\} \cup\left\{\hat{1}_{n+1}\right\} \in \mathcal{R}^{2(n+1)}$, где $x, y$ - элементы ранга $n$, и определим линейные операторы $\mathscr{O}_{0}, \mathscr{O}_{1}: \mathcal{R} \rightarrow \mathcal{R}$ формулами $\mathscr{O}_{0} T=2 T_{1}-T_{2}$, $\mathscr{O}_{1} T=T_{2}-T_{1}$. Для $T=\{\hat{0}=\hat{1}\}$ положим $T_{1}=\{\hat{0}, \hat{1}\}, T_{2}=2\{\hat{0}, \hat{1}\}$ и $\mathscr{O}_{0} T=0$, $\mathscr{O}_{1} T=\{\hat{0}, \hat{1}\}$.

Введем операторы $d_{k} \in \mathcal{L}_{-2 k}(\mathcal{R}), k \geqslant 0$, по формулам

$$
d_{k} T=\sum_{x \in T}[\hat{0}, x], \quad \rho(x)=\rho(T)-k .
$$

Обозначим через $\mathcal{D}(\mathcal{R})=\sum \mathcal{D}_{-2 k}(\mathcal{R}), k \geqslant 0$, градуированное кольцо, порожденное операторами $d_{k}$. 
Из конструкции прямого произведения частично упорядоченных множеств легко получить следующую формулу:

$$
d_{k}\left(T^{\prime} T^{\prime \prime}\right)=\sum_{i+j=k}\left(d_{i} T^{\prime}\right)\left(d_{j} T^{\prime \prime}\right),
$$

в частности, оператор $d=d_{1}$ является дифференцированием.

\section{4. *-кольцо многогранников.}

ОПРЕДЕЛЕНИЕ 1.36. Джойн многогранников определяет на кольце $\mathcal{P}$ билинейную операцию степени +2 . Она ассоциативна и коммутативна, поэтому группа $(\mathcal{P}, *)$ является ассоциативным коммутативным кольцом без единицы.

Используя соглашение $\varnothing * P=P=P * \varnothing$, добавим в это кольцо формальную единицу степени -2 как элемент, отвечающий пустому множеству $\varnothing$.

Обозначим абелеву группу $\mathbb{Z} \oplus \mathcal{P}$ через $\mathcal{R} \mathcal{P}$. Тогда $(\mathcal{R} \mathcal{P}, *)$ - коммутативное ассоциативное кольцо с единицей.

Многогранник $P^{n_{1}} * Q^{n_{2}}$ размерности $n_{1}+n_{2}+1$ имеет $f_{0, n_{1}}\left(P^{n_{1}}\right)+f_{0, n_{2}}\left(Q^{n_{2}}\right)$ вершин и $m_{1}+m_{2}$ гиперграней.

СлеДСТВИЕ 1.37. Кольцо $\mathcal{R} \mathcal{P}$ является триградуированным с градуировками $2(n+1), 2(m-n-1), 2(k-n-1)$, где $n=\operatorname{dim} P, m-$ число гиперграней, a $k=f_{0, n}-$ число вершин. Оператор двойственности * переводит многогранник с градуировками $(2(n+1), 2(m-n-1), 2(k-n-1))$ в многогранник с градуировками $(2(n+1), 2(k-n-1), 2(m-n-1))$.

Замечание 1.38. Напомним, что в кольце $\mathcal{P}$ многогранник $P$ имеет две градуировки: $2 n$ и $2(m-n)$. Так как $f_{0, n_{1}+n_{2}}\left(P^{n_{1}} \times Q^{n_{2}}\right)=f_{0, n_{1}}\left(P^{n_{1}}\right) \cdot f_{0, n_{2}}\left(Q^{n_{2}}\right)$, то число вершин не дает третьей градуировки в случае $\times$-произведения.

ОПРЕДЕЛЕНИЕ 1.39. Комбинаторный многогранник $P \neq \varnothing$ называется неразложимым относительно джойна, если его нельзя представить в виде джойна $P_{1} * P_{2}$ двух многогранников $P_{1} \neq \varnothing, P_{2} \neq \varnothing$.

УТВЕРЖДЕНИЕ 1.40. Колъцо $\mathcal{R P}$ является кольцом полиномов от неразложимых относительно джойна многогранников.

ДокАзАтЕЛьство. См. приложение А (утверждение А.2).

ОПРЕДЕЛЕНИЕ 1.41. Комбинаторный многогранник $P$ называется самодвойственнъм, если $P^{*}=P$.

ОПРЕДЕЛЕНИЕ 1.42. Обозначим через $\mathcal{S P}$ абелеву подгруппу в группе $\mathcal{R} \mathcal{P}$, состоящую из всех самодвойственных элементов.

Согласно утверждению 1.19 , группа $\mathcal{S P}$ является подкольцом в кольце $\mathcal{R} \mathcal{P}$.

ПримеР 1.43. Кольцо $\mathcal{S P}$ содержит подкольцо, порожденное симплексами и многоугольниками $M_{m}^{2}$.

Рассмотрим соответствие, переводящее многогранник $P$ в решетку его граней $L(P)$. Оно определяет мономорфизм абелевых групп $\mathcal{R} \mathcal{P} \rightarrow \mathcal{R}$. При этом $L(P * Q)=L(P) \cdot L(Q)$, и единица $\varnothing$ кольца $\mathcal{R} \mathcal{P}$ переходит в одноэлементное множество $\{\hat{0}=\hat{1}\}$, являющееся единицей кольца $\mathcal{R}$. Поэтому отображение $L$ является гомоморфизмом колец. Кроме того, $L\left(P^{*}\right)=L(P)^{*}$. 
Пусть $P$ - многогранник в $\mathcal{R} \mathcal{P}$. Тогда

$$
\Delta(L(P))=\sum_{\varnothing \subseteq F \subseteq P}[\varnothing, F] \otimes[F, P]=\sum_{\varnothing \subseteq F \subseteq P} L(F) \otimes L(P / F)
$$

и

$$
\varepsilon(L(P))= \begin{cases}1, & \text { если } P=\varnothing, \\ 0 & \text { в противном случае. }\end{cases}
$$

Тем самым мы доказали следующий факт.

УтВЕРЖДЕНИЕ 1.44. Отображение L задает структуру алгебры Хопфа $(\mathcal{R P}, \mu, \imath, \Delta, \varepsilon, \chi)$, где

- умножение $\mu: \mathcal{R P} \otimes \mathcal{R P} \rightarrow \mathcal{R P}$ задается джсойнм многогранников;

- единица $\imath: \mathbb{Z} \rightarrow \mathcal{R} \mathcal{P}$ переводит единицу $1 \in \mathbb{Z}$ в $\varnothing$

- коумножение $\Delta: \mathcal{R P} \rightarrow \mathcal{R P} \otimes \mathcal{R P}$ задается формулой

$$
\Delta(P)=\sum_{\varnothing \subseteq F \subseteq P} F \otimes P / F
$$

- коединица $\varepsilon: \mathcal{R P} \rightarrow \mathbb{Z}$ задается формулой

$$
\varepsilon(P)= \begin{cases}1, & \text { если } P=\varnothing, \\ 0 & \text { в противном случае }\end{cases}
$$

- антипод $\chi: \mathcal{R P} \rightarrow \mathcal{R P}$ действует по формуле

$$
\chi(P)=\sum_{k \geqslant 0} \sum_{\varnothing=F_{0} \subset F_{1} \subset \cdots \subset F_{k}=P}(-1)^{k}\left(F_{1} / F_{0}\right) *\left(F_{2} / F_{1}\right) * \cdots *\left(F_{k} / F_{k-1}\right) .
$$

Мы имеем: $\Delta\left(P^{*}\right)=* \otimes *\left(\tau_{\mathcal{R} \mathcal{P}, \mathcal{R} \mathcal{P}} \Delta P\right)$, т. е. операция $*$ является изоморфизмом алгебр Хопфа $\mathcal{R} \mathcal{P}$ и $\mathcal{R} \mathcal{P}^{\text {op cop }}$.

ПримеР 1.45. Пусть $\mathcal{B}$ - абелева подгруппа в кольце $\mathcal{R} \mathcal{P}$, порожденная пустым множеством $\varnothing$ и всеми симплексами $\Delta^{n}, n \geqslant 0$. Решетка граней $L\left(\Delta^{n}\right)$ представляет собой булеву алгебру $B_{n}=\{\hat{0}, \hat{1}\}^{n}=B_{1}^{n}$. В силу того, что $\Delta^{k} * \Delta^{l}=\Delta^{k+l+1}$, группа $\mathcal{B}$ является подкольцом. Положим $x=\Delta^{0}=\mathrm{pt}$. Тогда $\Delta^{n}=x^{n+1}$. Кроме того, $\Delta x=1 \otimes x+x \otimes 1, \chi(x)=-x$, поэтому подкольцо $\mathcal{B}$ является подалгеброй Хопфа, изоморфной алгебре Хопфа многочленов $\mathbb{Z}[x]$.

ЗАмЕчАНИЕ 1.46. Как мы увидим позже, самодвойственный многогранник может иметь грани, которые не являются самодвойственными. Поэтому кольцо $\mathcal{S P}$ не является подалгеброй Хопфа в $\mathcal{R} \mathcal{P}$.

ОПРЕДЕлЕниЕ 1.47. Триградуировка кольца $\mathcal{R} \mathcal{P}$ задает мультипликативный гомоморфизм

$$
\varepsilon_{\alpha, \beta, \gamma}: \mathcal{R P} \rightarrow \mathbb{Z}[\alpha, \beta, \gamma], \quad \varepsilon_{\alpha, \beta, \gamma}(P)=\alpha^{n+1} \beta^{m} \gamma^{k},
$$

где $n$ - размерность многогранника $P, m=m(P)$ - число его гиперграней, $k=f_{0, n}(P)$ - число вершин и $\operatorname{deg} \alpha=\operatorname{deg} \beta=\operatorname{deg} \gamma=2$.

Далее важную роль будет играть мультипликативный гомоморфизм $\varepsilon_{\alpha}=$ $\varepsilon_{\alpha, 1,1}: \mathcal{R P} \rightarrow \mathbb{Z}[\alpha]$. Отметим, что $\varepsilon_{0}=\varepsilon_{0,1,1}=\varepsilon$. 
1.5. Операторы на кольцах многогранников. $\times$-кольцо $\mathcal{P}$ и $*$-кольцо $\mathcal{R} \mathcal{P}$ имеют ряд общих свойств, при описании которых мы будем эти кольца обозначать единым символом $\mathrm{R}$. Положим $\mathrm{R}^{[2 n]}=\mathrm{R}^{0} \oplus \mathrm{R}^{2} \oplus \cdots \oplus \mathrm{R}^{2 n}$.

ОПРЕДЕЛЕНИЕ 1.48. Оператором граней $d_{k}, k \geqslant 0$, на кольце $\mathrm{R}$ называется оператор, сопоставляющий многограннику $P$ сумму всех его граней коразмерности $k$ :

$$
d_{k} P^{n}=\sum_{F^{n-k} \subseteq P^{n}} F^{n-k} .
$$

В частности, $d_{k} \varnothing=0, k \geqslant 1$, а $d_{n+1} P^{n}=\varnothing$ в кольце $\mathcal{R} \mathcal{P}$ и $d_{n+1} P^{n}=0$ в кольце $\mathcal{P}$.

Имеем: $d_{k} \in \mathcal{L}_{-2 k}(\mathrm{R})$ и $d_{k} L(P)=L\left(d_{k} P\right)$ при вложении $L: \mathcal{R} \mathcal{P} \rightarrow \mathcal{R}$.

ПримеР 1.49. Положим $d=d_{1}$. Тогда $d \mathrm{pt}=\varnothing$ в $\mathcal{R} \mathcal{P}$ и $d \mathrm{pt}=0$ в $\mathcal{P}$.

ОПРЕДЕЛЕНИЕ 1.50. Градуированное кольцо $\mathcal{D}(\mathrm{R}) \subset \mathcal{L}(\mathrm{R})$, порожденное операторами граней $d_{k}, k \geqslant 0$, называется алгеброй операторов граней. Имеем: $\mathcal{D}=\sum_{k=0}^{\infty} \mathcal{D}_{-2 k}$. Для каждого $n \geqslant 0$ группа $\mathrm{R}^{[2 n]} \subset \mathrm{R}$ замкнута относительно действия алгебры $\mathcal{D}(\mathrm{R})$. Соответствующая алгебра операторов обозначается через $\mathcal{D}\left(\mathrm{R}^{[2 n]}\right)$. Введем также группу $\mathcal{D}\left(\mathrm{R}^{2 n}, \mathrm{R}^{2(n-k)}\right)$ операторов $\mathrm{R}^{2 n} \rightarrow \mathrm{R}^{2(n-k)}$, порожденную операторами из $\mathcal{D}(\mathrm{R})$.

УтвеРЖДЕНИЕ 1.51. Для любых двух многогранников $P$ и $Q$ верны формульь

$$
\begin{array}{ll}
d(P \times Q)=(d P) \times Q+P \times(d Q) & \text { в кольие } \mathcal{P}, \\
d(P * Q)=(d P) * Q+P *(d Q) & \text { в кольие } \mathcal{R} \mathcal{P} .
\end{array}
$$

Таким образом, кольиа $\mathcal{P}$ и $\mathcal{R} \mathcal{P}$ являются дифберенииальными.

ДокАЗАтельство. Каждая гипергрань многогранника $P \times Q$ имеет вид $F \times Q$ или $P \times G$, где $F$ - гипергрань многогранника $P$, а $G$ - гипергрань многогранника $Q$ (в этом случае мы считаем, что у точки рt гиперграней нет). Поэтому

$$
d(P \times Q)=\sum_{F \subset P} F \times Q+\sum_{G \subset Q} P \times G=(d P) \times Q+P \times(d Q) .
$$

Каждая гипергрань многогранника $P * Q$ имеет вид $F * Q$ или $P * G$, где $F-$ гипергрань многогранника $P$, а $G$ - гипергрань многогранника $Q$ (в этом случае мы считаем, что точка pt имеет единственную гипергрань $\varnothing$, а у пустого множества $\varnothing$ гиперграней нет). Поэтому

$$
d(P * Q)=\sum_{F \subset P} F * Q+\sum_{G \subset Q} P * G=(d P) * Q+P *(d Q) .
$$

Пример 1.52. Так как $x P=\mathrm{pt} * P=C P$ - конус над $P$, то в $\mathcal{R} \mathcal{P}$ мы имеем: $d(C P)=d(\mathrm{pt} * P)=(d \mathrm{pt}) * P+\mathrm{pt} *(d P)=\varnothing * P+C(d P)=P+C(d P)$.

СлеДСтвиЕ 1.53. Оператор умножения на $x=\mathrm{pt}$ определяет на кольие $\mathcal{R} \mathcal{P}$ оператор конуса $C \in \mathcal{L}(\mathcal{R} \mathcal{P})$ степени 2 такой, что

$$
[d, C]=\mathrm{id} \text {. }
$$


В кольце $\mathcal{P}$ формула (9) верна для всех многогранников, кроме точки pt, для которой $d(C \mathrm{pt})=d I=2 \mathrm{pt}$. Так как $\mathrm{pt}+C(d \mathrm{pt})=\mathrm{pt}$, то вместо формулы $(9)$ в кольце $\mathcal{P}$ имеет место формула $d(C P)=C(d P)+P+\xi(P) \cdot \mathrm{pt}$.

Кольцо $\mathcal{P}$ не наследует структуры алгебры Хопфа из вложения абелевых групп $\mathcal{P} \subset \mathcal{R} \mathcal{P}$, однако для него определены единица $\jmath: \mathbb{Z} \rightarrow \mathcal{P}, \jmath(1)=\mathrm{pt}$, а также аугментация $\xi: \mathcal{P} \rightarrow \mathbb{Z}, \xi(\mathrm{pt})=1$ и $\xi(P)=0$, если $P \neq$ pt. Композиция

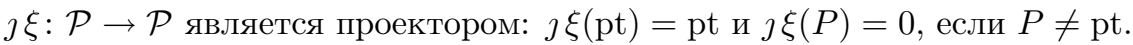

Таким образом, на кольце $\mathcal{P}$ выполнено соотношение:

$$
[d, C]=\mathrm{id}+\jmath \xi .
$$

ПримеР 1.54. Для симплексов $\Delta^{k}$ и $\Delta^{l}$ мы имеем: $d\left(\Delta^{k} * \Delta^{l}\right)=(k+1) \Delta^{k-1} * \Delta^{l}+(l+1) \Delta^{k} * \Delta^{l-1}=(k+l+2) \Delta^{k+l}=d \Delta^{k+l+1}$. Так как $\Delta^{k}=x^{k+1}$ и $d x^{k+1}=d \Delta^{k}=(k+1) \Delta^{k-1}=(k+1) x^{k}$, то дифференциальное кольцо $(\mathcal{R P}, d)$ содержит дифференциальное подкольцо $\left(\mathbb{Z}[x], \frac{d}{d x}\right)$.

Tеорема 1.55. (i) Пусть $P^{n} \in \mathcal{P}$ - многогранник. Соотношение

$$
f(\alpha, t)\left(d P^{n}\right)=\frac{\partial}{\partial t} f\left(P^{n}\right)(\alpha, t)
$$

выполнено тогда и только тогда, когда $P^{n}$ простой.

(ii) Пусть $\tilde{f}: \mathcal{P}_{\mathrm{s}} \rightarrow \mathbb{Z}[\alpha, t]$ - линейное отображение, удовлетворяющее условиям $\tilde{f}(d P)=\frac{\partial}{\partial t} \tilde{f}(P)$ и $\left.\tilde{f}\left(P^{n}\right)\right|_{t=0}=\alpha^{n}$ для любого простого многогранника $P^{n}$. Тогда $\tilde{f}(P)=f(\alpha, t)(P)$.

(iii) Для любого простого многогранника $P^{n}$ выполнено соотношение $f(\alpha, t)=f(-\alpha, \alpha+t)$.

ДокАЗАтЕЛЬСтво. (i) Пусть $P^{n}-$ простой многогранник. Тогда $f(\alpha, t)\left(d P^{n}\right)=\sum_{F^{n-1} \subset P} \sum_{G \subseteq F^{n-1}} \alpha^{\operatorname{dim} G} t^{n-1-\operatorname{dim} G}=\sum_{G \subset P} \sum_{F^{n-1} \supset G} \alpha^{\operatorname{dim} G} t^{n-1-\operatorname{dim} G}$. Каждая $k$-мерная грань простого многогранника лежит ровно в $n-k$ гипергранях. Поэтому $f(\alpha, t)\left(d P^{n}\right)=\sum_{G \subset P}(n-\operatorname{dim} G) \alpha^{\operatorname{dim} G} t^{n-1-\operatorname{dim} G}=\frac{\partial}{\partial t} f(\alpha, t)(P)$. Пусть теперь $f(\alpha, t)\left(d P^{n}\right)=\frac{\partial}{\partial t} f(\alpha, t)\left(P^{n}\right)$. Рассмотрим коэффициент при мономе $t^{n-1}$. Слева он равен $\sum_{F^{n-1}} f_{0, n-1}\left(F^{n-1}\right)$ - числу пар (вершина, гипергрань), а справа он равен $n f_{0, n}\left(P^{n}\right)$. Каждая вершина многогранника $P^{n}$ лежит не менее чем в $n$ гипергранях, поэтому $\sum_{F^{n-1}} f_{0, n-1}\left(F^{n-1}\right) \geqslant n f_{0, n}\left(P^{n}\right)$, причем равенство имеет место тогда и только тогда, когда $P^{n}$ простой.

(ii) Используем индукцию по размерности многогранника. Для $P^{0}=\mathrm{pt}$ имеем $\tilde{f}(\mathrm{pt})=1=f(\alpha, t)(\mathrm{pt})$. Предположим, что $\tilde{f}=f$ для всех многогранников размерности $k \leqslant n$. Тогда для любого $(n+1)$-мерного многогранника получаем: $\tilde{f}(\alpha, t)\left(d P^{n+1}\right)=f(\alpha, t)\left(d P^{n+1}\right)$, поэтому $\frac{\partial}{\partial t} \tilde{f}\left(P^{n+1}\right)=\frac{\partial}{\partial t} f\left(P^{n+1}\right)$. 
Таким образом, $\tilde{f}(\alpha, t)\left(P^{n+1}\right)=f(\alpha, t)\left(P^{n+1}\right)+c(\alpha)$. Полагая $t=0$, мы получаем $\alpha^{n+1}=\alpha^{n+1}+c(\alpha)$, поэтому $c(\alpha)=0$.

(iii) Снова используем индукцию по размерности многогранника. Мы имеем: $f(\alpha, t)(\mathrm{pt})=1=f(-\alpha, \alpha+t)(\mathrm{pt})$. Пусть соотношение верно для всех многогранников размерности $k \leqslant n$. Тогда для многогранника $P^{n+1}$ мы имеем: $f(\alpha, t)\left(d P^{n+1}\right)=f(-\alpha, \alpha+t)\left(d P^{n+1}\right)$. Таким образом, $\frac{\partial}{\partial t} f(\alpha, t)\left(P^{n+1}\right)=$ $\frac{\partial}{\partial t} f(-\alpha, \alpha+t)\left(P^{n+1}\right)$, поэтому $f(\alpha, t)\left(P^{n+1}\right)-f(-\alpha, \alpha+t)\left(P^{n+1}\right)=c(\alpha)$. Полагая $t=0$, получаем

$$
\alpha^{n+1}\left[1-\left((-1)^{n+1}+(-1)^{n} f_{n, 1}+\cdots+f_{0, n+1}\right)\right]=c(\alpha) .
$$

Согласно формуле Эйлера-Пуанкаре, мы имеем $f_{0, n+1}-f_{1, n}+\cdots+(-1)^{n} f_{n, 1}=$ $1+(-1)^{n}$, поэтому $c(\alpha)=0$.

Теорема доказана.

Таким образом, соотношение $f(\alpha, t)(d P)=\frac{\partial}{\partial t} f(\alpha, t)(P)$ позволяет доказывать утверждения о $f$-полиномах простых многогранников индукцией по размерности. В частности, доказать, что имеет место уравнение $f(\alpha, t)(P)=$ $f(-\alpha, \alpha+t)(P)$, равносильное соотношениям Дена-Соммервилля для чисел граней простых многогранников.

УТВЕРЖДЕНИЕ 1.56. Для любых двух многогранников $P, Q \in \mathcal{P}$ имеет место соотношение

$$
d(P \circ Q)=(d P) *(d Q) .
$$

Таким образом, отображение $d:(\mathcal{P}, \circ) \rightarrow \mathcal{R} \mathcal{P}, P \rightarrow d P$, является кольщевым гомоморфизмом.

ОПРедЕЛЕНИЕ 1.57. Каждому элементу $P \in \mathcal{P}$ соответствуют операторы $\times$-, о- и *-умножения: $L_{P}^{\times} Q=P \times Q, L_{P}^{\circ} Q=P \circ Q, L_{P}^{*} Q=P * Q$. Каждому элементу $P \in \mathcal{R P}$ соответствует оператор умножения $L_{P}^{*}: L_{P}^{*} Q=P * Q$.

УТВЕРЖДЕНИЕ 1.58. На кольце $\mathcal{P}$ выполнены соотношения

$$
\begin{aligned}
{\left[d, L_{P}^{\times}\right] } & =L_{d P}^{\times}, \\
d L_{P}^{\circ} & =L_{d P}^{*} d, \\
{\left[d, L_{P}^{*}\right] } & =L_{d P}^{*}+L_{P}^{\times} \jmath \xi,
\end{aligned}
$$

для $P=\mathrm{pt}$ имеем $L_{d P}^{*}=\mathrm{id}$.

На колъце $\mathcal{R} \mathcal{P}$ выполнено соотношение

$$
\left[d, L_{\stackrel{*}{*}}^{*}=L_{d P}^{*}\right.
$$

ОПРеДЕЛЕНИЕ 1.59. Соответствие $P \rightarrow B P$ определяет оператор бипирамидъ степени 2 на кольце $\mathrm{R}$. По определению $B \varnothing=0$.

На кольце $\mathcal{P}$ оператор конуса совпадает с $L_{\mathrm{pt}}^{*}$, а оператор бипирамиды совпадает с оператором $L_{I}^{\circ}$.

ЗАМЕЧАНИЕ 1.60. Легко видеть, что самодвойственный простой многогранник $P, \operatorname{dim} P>2$, комбинаторно эквивалентен симплексу. В то же время существует много примеров самодвойственных многогранников, не являющихся 
простыми. Такие примеры дает бесконечная серия $k$-угольных пирамид при $k \geqslant 4$.

Классическим примером является следующий правильный самодвойственный многогранник, гиперграни которого не являются самодвойственными.

ПримеР 1.61 (24-клетка). Пусть $Q$ - четырехмерный многогранник, задаваемый как выпуклая оболочка следующих 24 точек в $\mathbb{R}^{4}$ : концов 8 векторов $\pm \boldsymbol{e}_{i}$, $1 \leqslant i \leqslant 4$, и 16 точек вида $\left( \pm \frac{1}{2}, \pm \frac{1}{2}, \pm \frac{1}{2}, \pm \frac{1}{2}\right)$. Все гиперграни многогранника $Q$ являются октаэдрами.

Двойственный многогранник $Q^{*}$ может быть описан 24 неравенствами:

$$
\pm x_{i}+1 \geqslant 0 \text { для } 1 \leqslant i \leqslant 4 \quad \text { и } \quad \frac{1}{2}\left( \pm x_{1} \pm x_{2} \pm x_{3} \pm x_{4}\right)+1 \geqslant 0 .
$$

Каждое из этих неравенств обращается в равенство ровно в одной из указанных 24 точек, поэтому оно определяет опорную гиперплоскость, которая пересекается с многогранником $Q$ ровно в одной точке. Следовательно, многогранник $Q$ имеет ровно 24 вершины. Вершины многогранника $Q^{*}$ могут быть определены при помощи "процесса исключения переменных" в системе (16) (см. [7; раздел 1.2]). В результате мы получаем 24 точки вида $\pm \boldsymbol{e}_{i} \pm \boldsymbol{e}_{j}, 1 \leqslant i<j \leqslant 4$. Каждая опорная гиперплоскость, определяемая системой (16), содержит ровно 6 вершин многогранника $Q^{*}$, которые образуют октаэдр. Таким образом, оба многогранника $Q$ и $Q^{*}$ имеют 24 октаэдральные грани. На самом деле, оба эти многогранника дают пример правилъного четырехмерного многогранника, называемого 24-клеткой. Это единственный правильный самодвойственный многогранник размерности $>2$, отличный от симплекса. Подробности о 24-клетке и других правильных многогранниках можно найти в книге [16].

В этом примере $d Q=24 B I^{2}$, но $(d Q)^{*}=24 I^{3}$. Таким образом, кольцо $\mathcal{S P}$ не является дифберенциальным подкольцом в кольце $\mathcal{R} \mathcal{P}$. Однако существует другой способ определить дифференцирование на кольце $\mathcal{S P}$. Инволюция * является кольцевым гомоморфизмом, а оператор $d$ - дифференцированием на кольце $\mathcal{R} \mathcal{P}$, поэтому оператор $\delta=* d *$ также является дифференцированием на $\mathcal{R} \mathcal{P}$.

УТВЕРЖДЕНИЕ 1.62. Оператор $d+\delta$ является дифференцированием кольu, $\boldsymbol{S} \mathcal{S P}$.

ДоказАтельство. По определению $P=P^{*}$ для любого элемента $P \in \mathcal{S P}$, поэтому

$$
((d+\delta) P)^{*}=*(d+* d *) P=* d P+* * d P=* d * P+d P=(\delta+d) P .
$$

УтВеРЖДЕНИЕ 1.63. Для любых двух многогранников $P$ и $Q$ верны формуль

$$
\begin{aligned}
& d_{k}(P \times Q)=\sum_{i+j=k}\left(d_{i} P\right) \times\left(d_{j} Q\right) \quad \text { в кольце } \mathcal{P}, \\
& d_{k}(P * Q)=\sum_{i+j=k}\left(d_{i} P\right) *\left(d_{j} Q\right) \quad \text { в кольце } \mathcal{R} \mathcal{P} .
\end{aligned}
$$


ДокаЗАТЕЛЬСтво. Мы имеем:

$$
\begin{aligned}
d_{k}\left(P^{n} \times Q^{r}\right) & =\sum_{F^{n-i} \subseteq P^{n}, G^{r-j} \subseteq Q^{r}, i+j=k} F^{n-i} \times G^{r-j} \\
& =\sum_{i+j=k} \sum_{F^{n-i} \subseteq P^{n}} F^{n-i} \times\left(\sum_{G^{r-j} \subseteq Q^{r}} G^{r-j}\right) \\
& =\sum_{i+j=k}\left(\sum_{F^{n-i} \subseteq P^{n}} F^{n-i}\right) \times\left(\sum_{G^{r-j} \subseteq Q^{r}} G^{r-j}\right)=\sum_{i+j=k}\left(d_{i} P\right) \times\left(d_{j} Q\right) .
\end{aligned}
$$

Такое же рассуждение годится и для многогранника $P^{n} * Q^{r}$. Единственным различием является то, что джойн $P^{n} * Q^{r}$ имеет грани вида $\varnothing * G^{r-j}, i=n+1$, $i+j=k$, и $F^{n-i} * \varnothing, j=r+1, i+j=k$. Тогда $\varnothing=d^{n+1} P^{n}=d^{r+1} Q^{r}$.

СледСтвие 1.64. На кольце $\mathcal{P}$ имеем: $d_{k} L_{P}^{\times}=\sum_{i=0}^{k} L_{d_{i} P}^{\times} d_{k-i}$. На кольце $\mathcal{R} \mathcal{P}$ имеем: $d_{k} L_{P}^{*}=\sum_{i=0}^{k} L_{d_{i}}^{*} d_{k-i}$. Таким образом, любой оператор в кольце $\mathrm{RD}(\mathrm{R})$, порожденном операторами из кольца $\mathcal{D}(\mathrm{R})$ и операторами $\left\{L_{P}^{\mu}, P \in \mathrm{R}\right\}$, где $\mu$ - умножение в кольце R, может быть представлен в виде

$$
\sum_{P, \omega} L_{P}^{\mu} D_{\omega}, \quad P \in \mathrm{R}, \quad D_{\omega} \in \mathcal{D}(\mathrm{R})
$$

ОПРЕДЕЛЕНИЕ 1.65. Введем оператор $\Phi: \mathrm{R} \rightarrow \mathrm{R}[t], \Phi \in \mathcal{D}(\mathrm{R})[[t]]$, по формуле

$$
\Phi(t)=1+d t+d_{2} t^{2}+\cdots+d_{k} t^{k}+\cdots .
$$

УтВЕРЖДЕНИЕ 1.66. Отображение $\Phi(t): \mathrm{R} \rightarrow \mathrm{R}[t]$ является гомоморфизмом колеи.

ДокАЗАТЕЛьство. Пусть - - умножение в R. Пользуясь формулами (17) и (18), для любых двух многогранников $P^{n_{1}}$ и $Q^{n_{2}}$ получаем:

$$
\begin{aligned}
\Phi(t)\left(P^{n_{1}} \cdot Q^{n_{2}}\right) & =\sum_{k=0}^{\infty} d_{k}\left(P^{n_{1}} \cdot Q^{n_{2}}\right) t^{k}=\sum_{k=0}^{\infty}\left(\sum_{i+j=k}\left(d_{i} P^{n_{1}}\right) \cdot\left(d_{j} Q^{n_{2}}\right)\right) t^{k} \\
& =\sum_{i, j \geqslant 0}\left(d_{i} P^{n_{1}} t^{i}\right) \cdot\left(d_{j} Q^{n_{2}} t^{j}\right)=\left(\sum_{i=0}^{\infty} d_{i} P^{n_{1}} t^{i}\right) \cdot\left(\sum_{j=0}^{\infty} d_{j} Q^{n_{2}} t^{j}\right) \\
& =\left(\Phi(t) P^{n_{1}}\right) \cdot\left(\Phi(t) Q^{n_{2}}\right) .
\end{aligned}
$$

Наконец, $\Phi(t)$ pt $=$ pt в кольце $\mathcal{P}$ и $\Phi(t) \varnothing=\varnothing$ в кольце $\mathcal{R} \mathcal{P}$, поэтому отображение $\Phi(t)$ является гомоморфизмом колец $\mathrm{R} \rightarrow \mathrm{R}[t]$.

УтвеРЖДЕНИЕ 1.67. На колъце $\mathrm{R}$ верно соотношение $\Phi(-t) \Phi(t)=1$, m.е.

$$
d_{n}-d d_{n-1}+\cdots+(-1)^{n-1} d_{n-1} d+(-1)^{n} d_{n}=0, \quad n \geqslant 1 .
$$

ДоказАтельство. Для $\varnothing \in \mathcal{R} \mathcal{P}$ утверждение, очевидно, верно. Пусть pt $\in \mathcal{P}$, тогда

$$
\Phi(-t) \Phi(t) \mathrm{pt}=(1-d t+\cdots)(\mathrm{pt}+\varnothing t)=\mathrm{pt}-\varnothing t+\varnothing t=\mathrm{pt} .
$$


Имеем $\Phi(-t) \Phi(t)=\sum\left(\sum(-1)^{i} d_{i} d_{k-i}\right) t^{k}$. Пусть $n>0$ и $k>n+1$, тогда для многогранника $P^{n}$ коэффициент при $t^{k}$ ряда $\Phi(-t) \Phi(t) P^{n}$ равен нулю. Если $k=0$, то он равен $P^{n}$. Для $1 \leqslant k \leqslant n$ мы получаем:

$$
\begin{gathered}
\left(d d_{k-1}-d_{2} d_{k-2}+\cdots+(-1)^{k-2} d_{k-1} d\right) P^{n} \\
=\sum_{F^{n-k} \subset P^{n}}\left(\sum_{F^{n-k+1} \supset F^{n-k}} 1-\sum_{F^{n-k+2} \supset F^{n-k}} 1+\cdots\right. \\
\left.\quad+(-1)^{k-2} \sum_{F^{n-1} \supset F^{n-k}} 1\right) F^{n-k} \\
=\sum_{F^{n-k} \subset P^{n}}\left(f_{0, k-1}\left(P^{n} / F^{n-k}\right)-f_{1, k-2}\left(P^{n} / F^{n-k}\right)+\cdots\right. \\
\left.\quad+(-1)^{k-2} f_{k-2,1}\left(P^{n} / F^{n-k}\right)\right) F^{n-k} .
\end{gathered}
$$

Для $(k-1)$-мерного многогранника $P^{n} / F^{n-k}$ верна формула Эйлера-Пуанкаре $f_{0, k-1}\left(P^{n} / F^{n-k}\right)-f_{1, k-2}\left(P^{n} / F^{n-k}\right)+\cdots+(-1)^{k-2} f_{k-2,1}\left(P^{n} / F^{n-k}\right)=1+(-1)^{k}$. Тогда $\left(d d_{k-1}-d_{2} d_{k-2}+\cdots+(-1)^{k-2} d_{k-1} d\right) P^{n}=\left(1+(-1)^{k}\right) d_{k} P^{n}$, поэтому $\left(\sum(-1)^{i} d_{i} d_{k-i}\right) P^{n}=0$. Коэффициент при $t^{n+1}$ равен нулю в кольце $\mathcal{P}$ и $\left(1-f_{0, n}+f_{1, n-1}+\cdots+(-1)^{n} f_{n-1,1}+(-1)^{n+1}\right) \varnothing$ в кольце $\mathcal{R} \mathcal{P}$. Из формулы Эйлера-Пуанкаре следует, что последнее выражение тоже равно нулю.

УТВЕРЖДЕНИЕ 1.68. Имеют место формуль:

$$
\begin{array}{ll}
\xi_{-\alpha} \Phi(\alpha)=\xi_{\alpha} & \text { накольце } \mathcal{P}, \\
\varepsilon_{-\alpha} \Phi(\alpha)=\varepsilon_{0} & \text { на кольце } \mathcal{R} \mathcal{P} .
\end{array}
$$

ДокАЗАТЕЛЬСтво. Так как $\xi_{\alpha} d_{k} P^{n}=f_{n-k, k}\left(P^{n}\right) \alpha^{n-k}$, то

$$
\begin{aligned}
\xi_{-\alpha} \Phi(\alpha) P^{n} & =(-\alpha)^{n}+(-\alpha)^{n-1} f_{n-1,1} \alpha+\cdots+f_{0, n} \alpha^{n} \\
& =\left(\sum_{i=0}^{n}(-1)^{i} f_{i, n-i}\right) \alpha^{n}=\alpha^{n}=\xi_{\alpha} P^{n}
\end{aligned}
$$

в силу формулы Эйлера-Пуанкаре.

С другой стороны, на кольце $\mathcal{R} \mathcal{P}$ мы имеем: $\varepsilon_{\alpha} d_{k} P^{n}=f_{n-k, k}\left(P^{n}\right) \alpha^{n-k+1}$, поэтому

$$
\varepsilon_{-\alpha} \Phi(\alpha) P^{n}=(-\alpha)^{n+1}+(-\alpha)^{n} f_{n-1,1} \alpha+\cdots+(-\alpha) f_{0, n} \alpha^{n}+\alpha^{n+1} .
$$

Из формулы Эйлера-Пуанкаре следует, что это выражение равно нулю для всех многогранников, кроме $\varnothing$. Таким образом, $\varepsilon_{-\alpha} \Phi(\alpha)=\varepsilon_{0}$.

УТВЕРЖДЕНИЕ 1.69. Колъцо простых многогранников $\mathcal{P}_{\mathrm{s}}$ замкнуто относительно действия алгебры операторов $\mathcal{D}(\mathcal{P})$, которая на этом кольце действует как алгебра разделенных степеней, т.е. $\left.\Phi(t)\right|_{\mathcal{P}_{\mathrm{s}}}=\left.e^{d t}\right|_{\mathcal{P}_{\mathrm{s}}} u$, более подробно,

$$
\left.k ! d_{k}\right|_{\mathcal{P}_{\mathrm{s}}}=\left.\left.d^{k}\right|_{\mathcal{P}_{\mathrm{s}}} \quad u \quad d_{k} d_{l}\right|_{\mathcal{P}_{\mathrm{s}}}=\left.\left(\begin{array}{c}
k+l \\
k
\end{array}\right) d_{k+l}\right|_{\mathcal{P}_{\mathrm{s}}} .
$$


Доказательство. Пусть $P^{n}-$ простой многогранник. Тогда все его грани тоже являются простыми многогранниками. Для любого $k>0$ имеем

$$
d^{k} P^{n}=\sum_{F^{n-k} \subset P^{n}} F^{n-k}\left(\sum_{P^{n} \supset G^{n-1} \supset \cdots \supset G^{n-k+1} \supset F^{n-k}} 1\right),
$$

где $P^{n} \supset G^{n-1} \supset \cdots \subset G^{n-k+1} \supset F^{n-k}-$ всевозможные цепочки граней, содержащие грань $F^{n-k}$. Каждая гранная фигура $P / F^{n-k}$ простого многогранника является $(k-1)$-мерным симплексом, поэтому число таких цепочек равно $k !$. Таким образом, $d^{k} P^{n}=k ! d_{k} P^{n}$.

Заметим, что абелева подгруппа $\mathcal{P}_{\mathrm{s}} \subset \mathcal{R} \mathcal{P}$ не замкнута относительно действия алгебры $\mathcal{D}(\mathcal{R} \mathcal{P})$. Если добавить пустое множество $\varnothing$, то группа $\mathbb{Z} \varnothing \oplus \mathcal{P}_{\mathrm{s}}$ уже будет замкнута относительно этого действия, но формула (22) для нее, вообще говоря, не выполнена. Дело в том, что для простого многогранника $P^{n}$ мы имеем: $k ! d_{k} P^{n}=d^{k} P^{n}$ для $1 \leqslant k \leqslant n$; но $(n+1) ! d_{n+1} P^{n}=(n+1) ! \varnothing$, в то время как

$$
d^{n+1} P^{n}=d\left(d^{n} P^{n}\right)=d\left(n ! d_{n} P^{n}\right)=d n ! f_{0, n}\left(P^{n}\right) \mathrm{pt}=n ! f_{0, n}\left(P^{n}\right) \varnothing .
$$

Следовательно $k ! d_{k} P^{n}=d^{k} P^{n}$ для всех $k, 1 \leqslant k \leqslant n$ тогда и только тогда, когда многогранник $P^{n}$ является простым, при этом равенство верно для $k=n+1$ тогда и только тогда, когда он имеет $n+1$ вершину, т. е. является симплексом.

ПримеР 1.70. Подкольцо $\mathbb{Z}[x] \subset \mathcal{R} \mathcal{P}, x=\mathrm{pt}$, замкнуто относительно действия алгебры $\mathcal{D}(\mathcal{R} \mathcal{P})$, при этом $k ! d_{k}=d^{k}=\frac{d^{k}}{d x^{k}}$.

Рассмотрим еще одно подкольцо, на котором реализуется представление алгебры $\mathcal{D}(\mathcal{R} \mathcal{P})$. Пусть $x=$ pt и $y=M_{4}^{2}-M_{3}^{2}=I^{2}-x^{3}$. Переменные $x$ и $y$ алгебраически независимы, поэтому они порождают кольцо полиномов $\mathbb{Z}[x, y]$ и

$$
d=(d x) \frac{\partial}{\partial x}+(d y) \frac{\partial}{\partial y}=\frac{\partial}{\partial x}+x^{2} \frac{\partial}{\partial y} .
$$

Положим

$$
s(t)=s_{1} t+s_{2} t^{2}+s_{3} t^{3}+\cdots=\log \Phi(t)=\sum_{k=1}^{\infty} \frac{(-1)^{k-1}}{k}(\Phi(t)-1)^{k} \in \mathcal{D}(\mathcal{R P}) \otimes \mathbb{Q} .
$$

Например, $s_{1}=d, s_{2}=0, s_{3}=d_{3}-(1 / 6) d^{3}$. Из утверждения 1.67 непосредственно следует, что $s(-t)=-s(t)$ (т. е. $s_{2 k}=0$ для всех $k \geqslant 1$ ), а позже мы покажем, что $s(t)(P * Q)=(s(t) P) * Q+P *(s(t) Q)$, т. е. каждый оператор $s_{k}$ является дифференцированием кольца $\mathcal{R} \mathcal{P}$.

Тогда $\Phi(t)=\exp s(t)=\sum \frac{1}{k !} s(t)^{k}$ и $s(t)=(s(t) x) \frac{\partial}{\partial x}+(s(t) y) \frac{\partial}{\partial y}$. Так как каждый оператор $s_{k}$ имеет степень $-2 k$, то $s_{k} x=0$ при $k \geqslant 2$ и $s_{k} y=0$ при $k \geqslant 4$. В то же время $s_{1} x=1, s_{1} y=x^{2}, s_{2} y=0, s_{3} y=-1 / 3$, поэтому $s(t) x=t$ и $s(t) y=t\left(x^{2}-t^{2} / 3\right)$. Таким образом,

$$
s(t)=t\left(\frac{\partial}{\partial x}+\left(x^{2}-\frac{1}{3} t^{2}\right) \frac{\partial}{\partial y}\right) \quad \text { и } \quad \Phi(t)=\exp \left(t\left(\frac{\partial}{\partial x}+\left(x^{2}-\frac{1}{3} t^{2}\right) \frac{\partial}{\partial y}\right)\right) .
$$

В частности, $d_{3}=\frac{1}{6} d^{3}-\frac{1}{3} \frac{\partial}{\partial y}=\frac{1}{6}\left(\frac{\partial}{\partial x}+x^{2} \frac{\partial}{\partial y}\right)^{3}-\frac{1}{3} \frac{\partial}{\partial y}$. 
ОПредЕЛЕНиЕ 1.71. Введем операторы $\mathscr{A}, \mathscr{D}: \mathrm{R} \rightarrow \mathrm{R}$, задаваемые формулами: $\mathscr{A}=2 C-B$ и $\mathscr{D}=[B, C]=B C-C B$.

УтВЕРЖДЕНИЕ 1.72. (i) На колъце $\mathcal{P}$ выполнены соотношения:

1) $\left.\Phi(t) C=(C+t) \Phi(t)+t \jmath \xi_{t} ; 2\right) \Phi(t) B=(B-2 C-t)+(2 C+t) \Phi(t)+2 t \jmath \xi_{t}$.

(ii) На кольие $\mathcal{R} \mathcal{P}$ выполнены соотношения:

1) $\Phi(t) C=(C+t) \Phi(t) ; 2) \Phi(t) B=(B-2 C-t)+(2 C+t) \Phi(t)$.

(iii) На кольие $\mathrm{R}$ выполнены соотношения:

1) $\Phi(t) \mathscr{A}=\mathscr{A}+t+t \Phi(t)$; 2) $\Phi(t) \mathscr{D}=\mathscr{D}+\mathscr{A} t+t^{2}$.

ДокАЗАТЕЛЬСтво. (i) Для точки мы имеем: $\Phi(t) \mathrm{pt}=\mathrm{pt}$ и

$$
\begin{aligned}
\Phi(t) C \mathrm{pt} & =\Phi(t) I=I+(2 \mathrm{pt}) t=C \mathrm{pt}+(\mathrm{pt}) t+(\mathrm{pt}) t=(C+t) \Phi(t) \mathrm{pt}+t \jmath \xi_{t}(\mathrm{pt}), \\
\Phi(t) B \mathrm{pt} & =\Phi(t) I=I+(2 \mathrm{pt}) t=B \mathrm{pt}-(2 C+t) \mathrm{pt}+(2 C+t) \mathrm{pt}+2 t \jmath \xi_{t}(\mathrm{pt}) \\
& =B \mathrm{pt}-(2 C+t) \mathrm{pt}+(2 C+t) \Phi(t) \mathrm{pt}+2 t \jmath \xi_{t}(\mathrm{pt}) \\
& =\left[(B-2 C-t)+(2 C+t) \Phi(t)+2 t \jmath \xi_{t}\right] \mathrm{pt} .
\end{aligned}
$$

Пусть $P-n$-мерный многогранник, $n \geqslant 1$. Тогда

$$
\begin{aligned}
\Phi(t) C P= & C P+(C d P+P) t+\cdots+\left(C d_{n} P+d_{n-1} P\right) t^{n}+\left(1+d_{n} P\right) t^{n+1} \\
= & C \Phi(t) P+t \Phi(t) P+t \xi_{t}(P), \\
\Phi(t) B P= & B P+(2 C d P) t+\left(2 C d_{2} P+d P\right) t^{2}+\cdots+\left(2 C d_{n} P+d_{n-1} P\right) t^{n} \\
& \quad+\left(2+d_{n} P\right) t^{n+1}=(B-2 C-t) P+2 C \Phi(t) P+t \Phi(t) P+2 t \jmath \xi_{t}(P) .
\end{aligned}
$$

(ii) Оператор $C$ на кольце $\mathcal{R} \mathcal{P}$ задается умножением на $x=\mathrm{pt}$, отображение $\Phi(t): \mathcal{R} \mathcal{P} \rightarrow \mathcal{R} \mathcal{P}[t]$ - кольцевой гомоморфизм, поэтому

$$
\Phi(t) C P=(\Phi(t) x) \cdot(\Phi(t) P)=(x+2 t \varnothing) \cdot(\Phi(t) P)=(C+2 t) \Phi(t) P .
$$

Для $P=\varnothing$ имеем:

$$
\Phi(t) B \varnothing=0, \quad((B-2 C-t)+(2 C+t) \Phi(t)) \varnothing=-2 \mathrm{pt}-\varnothing t+2 \mathrm{pt}+\varnothing t=0 .
$$

Пусть $\operatorname{dim} P=n \geqslant 0$. Тогда

$$
\begin{aligned}
\Phi(t) B P=B P & +(2 C d P) t+\left(2 C d_{2} P+d P\right) t^{2}+\cdots+\left(2 C d_{n} P+d_{n-1} P\right) t^{n} \\
& +\left(2 C \varnothing+d_{n} P\right) t^{n+1}+\varnothing t^{n+2}=(B-2 C-t) P+2 C \Phi(t) P+t \Phi(t) P .
\end{aligned}
$$

(iii) Формула 1) непосредственно следует из формул первых двух частей утверждения. На кольце $\mathcal{P}$ имеем: $\jmath \xi_{t} B=t \jmath \xi_{t}=\jmath \xi_{t} C=\jmath \xi_{t} \mathscr{A}$, поэтому

$$
\begin{aligned}
\Phi(t) \mathscr{D} & =\Phi(t)(B C-C B)=\Phi(t)(C \mathscr{A}-\mathscr{A} C) \\
& =\left[(C+t) \Phi(t)+t \jmath \xi_{t}\right] \mathscr{A}-[\mathscr{A}+t+t \Phi(t)] C \\
& =(C+t)(\mathscr{A}+t+t \Phi(t))+t^{2} \jmath \xi_{t}-\left[\mathscr{A} C+C t+t(C+t) \Phi(t)+t^{2} \jmath \xi_{t}\right] \\
& =C \mathscr{A}-\mathscr{A} C+\mathscr{A} t+t^{2}=\mathscr{D}+\mathscr{A} t+t^{2} .
\end{aligned}
$$

На кольце $\mathcal{R} \mathcal{P}$ имеем:

$$
\begin{aligned}
\Phi(t) \mathscr{D} & =\Phi(t)(C \mathscr{A}-\mathscr{A} C)=[(C+t) \Phi(t)] \mathscr{A}-[\mathscr{A}+t+t \Phi(t)] C \\
& =(C+t)(\mathscr{A}+t+t \Phi(t))-[\mathscr{A} C+C t+t(C+t) \Phi(t)] \\
& =C \mathscr{A}-\mathscr{A} C+\mathscr{A} t+t^{2}=\mathscr{D}+\mathscr{A} t+t^{2} .
\end{aligned}
$$


СЛЕДСТВИЕ 1.73. На кольце $\mathrm{R}$ имеем:

$$
\begin{gathered}
d \mathscr{D}=\mathscr{A}, \quad d_{2} \mathscr{D}=1, \quad d_{k} \mathscr{D}=0, \quad k \geqslant 3 ; \\
d \mathscr{A}=2, \quad d_{k} \mathscr{A}=d_{k-1}, \quad k \geqslant 2 .
\end{gathered}
$$

1.6. Флаговые векторы. Введем на множестве $2^{[0, n-1]}$ всех подмножеств множества $[0, n-1]=\{0,1, \ldots, n-1\}$ полный порядок следующим условием:

Для двух множеств $S_{1}=\left\{a_{1}, \ldots, a_{k}\right\}, a_{1}<\cdots<a_{k}$, и $S_{2}=\left\{b_{1}, \ldots, b_{l}\right\}$, $b_{1}<\cdots<b_{l}$, мы имеем $S_{1}<S_{2}$ тогда и только тогда, когда слово $a_{k} a_{k-1} \ldots a_{1}$ меньше слова $b_{l} b_{l-1} \ldots b_{1}$ в лексикографическом порядке.

Например, $\varnothing<\{0\}<\{1\}<\{0,1\}<\{0,1,2\}<\{3\}<\{0,1,2,3\}$.

Для множества $S \subseteq[0, n-1]$ и числа $k \geqslant 0$ положим $S+k=\{s+k$ : $s \in S\} \subseteq[0, n+k-1]$.

ОПРЕДЕЛЕНИЕ 1.74. Пусть $P^{n}-n$-мерный многогранник и $S=\left\{a_{1}, \ldots, a_{k}\right\}$, $a_{1}<\cdots<a_{k},-$ подмножество в $[0, n-1]$. Число возрастающих цепочек граней

$$
F^{a_{1}} \subset F^{a_{2}} \subset \cdots \subset F^{a_{k}}, \quad \operatorname{dim} F^{a_{i}}=a_{i},
$$

называется флаговым числом и обозначается $f_{S}=f_{\left\{a_{1}, \ldots, a_{k}\right\}}$. Положим $f_{\varnothing}=1$. Например, число $f_{\{i\}}$ равно числу $f_{i, n-i}$ граней размерности $i$, которое мы уже определили ранее. Длину $k$ цепочки обозначим через $l(S)$. Тогда $0 \leqslant$ $l(S) \leqslant n$. Набор $\left\{f_{S}\right\}$ всех флаговых чисел многогранника называется его флаговым вектором.

Для множества $S=\left\{a_{1}, \ldots, a_{k}\right\} \subseteq[0, n-1]$ положим $a_{0}=-1, a_{k+1}=n$ и

$$
f_{\left\{-1, a_{1}, \ldots, a_{k}\right\}}=f_{\left\{-1, a_{1}, \ldots, a_{k}, n\right\}}=f_{\left\{a_{1}, \ldots, a_{k}, n\right\}}=f_{\left\{a_{1}, \ldots, a_{k}\right\}} .
$$

Это соглашение соответствует тому, что пустое множество $\varnothing$ является единственной гранью размерности -1 и содержится в любой другой грани многогранника $P^{n}$, а сам многогранник является единственной гранью размерности $n$ и содержит любую другую грань.

Для элемента $P=\sum \lambda_{i} P_{i}^{n} \in \mathcal{P}^{2 n}=\mathcal{R} \mathcal{P}^{2(n+1)}, n \geqslant 0$, определим флаговое число $f_{S}$ по формуле $f_{S}(P)=\sum \lambda_{i} f_{S}\left(P_{i}^{n}\right)$.

УТВЕРЖДЕНИЕ 1.75. Для любого многогранника $P^{n}, n \geqslant 0, u$ множества $S=\left\{a_{1}, \ldots, a_{k}\right\} \subseteq[0, n-1]$ ми имеем:

$f_{S}\left(P^{n}\right)=\xi_{1} d_{a_{2}-a_{1}} d_{a_{3}-a_{2}} \cdots d_{n-a_{k}} P^{n}=f_{\{-1\} \cup S}\left(P^{n}\right)=\varepsilon d_{a_{1}+1} d_{a_{2}-a_{1}} \cdots d_{n-a_{k}} P^{n}$.

УтВеРЖДЕНИЕ 1.76. Для многогранника $P$ размерности $n-2 \geqslant 0$ и множества $S=\left\{a_{1}, \ldots, a_{k}\right\} \subseteq[0, n-2]$ имеем:

$$
f_{S}(\mathscr{D} P)=\left\{\begin{array}{ll}
0, & n-2 \notin S, \\
f_{S \backslash\{n-2\}}(P), & n-2 \in S ;
\end{array} \quad f_{S}(\mathscr{A} P)= \begin{cases}f_{S}(P), & n-2 \notin S, \\
2 f_{S \backslash\{n-2\}}(P), & n-2 \in S .\end{cases}\right.
$$

ДокАЗАТЕЛЬство. Для $S=\varnothing$ утверждения верны. Пусть $k \geqslant 1$. Имеем: $f_{S}(\mathscr{D} P)=\xi_{1}\left(d_{a_{2}-a_{1}} \cdots d_{n-a_{k}} \mathscr{D} P\right)$ и $f_{S}(\mathscr{A} P)=\xi_{1}\left(d_{a_{2}-a_{1}} \cdots d_{n-1-a_{k}} \mathscr{A} P\right)$. Если $n-a_{k} \geqslant 3$, то $d_{n-a_{k}} \mathscr{D}=0$ и $d_{n-1-a_{k}} \mathscr{A}=d_{n-2-a_{k}}$, поэтому $f_{S}(\mathscr{D} P)=0$ 
и $f_{S}(\mathscr{A} P)=f_{S}(P)$. Если же $n-a_{k}=2$, то $d_{n-a_{k}} \mathscr{D}=1$ и $d_{n-1-a_{k}} \mathscr{A}=2$, поэтому

$$
\begin{aligned}
f_{S}(\mathscr{D} P) & =\xi_{1} d_{a_{2}-a_{1}} \cdots d_{a_{k}-a_{k-1}} d_{n-a_{k}} \mathscr{D} P \\
& =\xi_{1} d_{a_{2}-a_{1}} \cdots d_{n-2-a_{k-1}} P=f_{S \backslash\{n-2\}}(P), \\
f_{S}(\mathscr{A} P) & =\xi_{1} d_{a_{2}-a_{1}} \cdots d_{a_{k}-a_{k-1}} d_{n-1-a_{k}} \mathscr{A} P \\
& =2 \xi_{1} d_{a_{2}-a_{1}} \cdots d_{n-2-a_{k-1}} P=2 f_{S \backslash\{n-2\}}(P) .
\end{aligned}
$$

В работе [17] доказано, что флаговые векторы многогранников удовлетворяют обобщенным соотношениям Дена-Соммервилля (соотношениям БайерБиллера).

Tеорема 1.77 (см. [17; теорема 2.1]). Рассмотрим $n$-мерный многогранник $P^{n}$ и множество $S \subset\{0,1, \ldots, n-1\}$. Пусть $\{i, k\} \subseteq S \cup\{-1, n\}$, где $i<k-1 u S \cap\{i+1, \ldots, k-1\}=\varnothing$, тогда

$$
\sum_{j=i+1}^{k-1}(-1)^{j-i-1} f_{S \cup\{j\}}=\left(1-(-1)^{k-i-1}\right) f_{S} .
$$

ОПРЕДЕЛЕНИЕ 1.78. Пусть $\Psi^{n}, n \geqslant 2,-$ множество всех подмножеств $S \subseteq$ $\{0,1, \ldots, n-2\}$, которые не содержат двух последовательных чисел. Положим $\Psi^{1}=\Psi^{0}=\{\varnothing\}$.

Можно показать по индукции, что мощность множества $\Psi^{n}$ равна $n$-му числу Фибоначчи $c_{n}\left(c_{0}=1, c_{1}=1, c_{n+1}=c_{n}+c_{n-1}, n \geqslant 1\right)$.

ОПРЕДЕЛЕНИЕ 1.79. Пусть $\Omega^{n}, n \geqslant 1,-$ множество всех $n$-мерных выпуклых многогранников, которые получаются применением к пустому множеству $\varnothing$ слов из операторов $B$ и $C$, которые не содержат двух последовательных операторов $B$ и оканчиваются на $C^{2}$. Мы будем отождествлять многогранники из множества $\Omega^{n}$ со словами, которые им соответствуют. Определим на множестве слов из букв $B$ и $C$ лексикографический порядок, положив $C<B$.

Имеем: $\Omega^{1}=\left\{C^{2}\right\}, \Omega^{2}=\left\{C^{3}, B C^{2}\right\}$, при этом $C^{2}<C^{3}<B C^{2}$.

Каждое слово длины $n+1$ из множества $\Omega^{n}$ имеет вид либо $C Q, Q \in \Omega^{n-1}$, либо $B C Q, Q \in \Omega^{n-2}$, так что мощность множества $\Omega^{n}$ удовлетворяет уравнению для чисел Фибоначчи $\left|\Omega^{n}\right|=\left|\Omega^{n-1}\right|+\left|\Omega^{n-2}\right|$. Так как $\left|\Omega^{1}\right|=1,\left|\Omega^{2}\right|=2$, то $\left|\Omega^{n}\right|=c_{n}, n \geqslant 1$.

ЗАмечание 1.80. Мы видим, что $\left|\Omega^{n}\right|=\left|\Psi^{n}\right|=c_{n}$. Существует естественное взаимно однозначное соответствие между множествами $\Omega^{n}$ и $\Psi^{n}$, при котором слово $C^{n+1-a_{k}} B C^{a_{k}-a_{k-1}-1} B \cdots B C^{a_{1}-1}$ переходит в множество $\left\{a_{1}-3\right.$, $\left.\ldots, a_{k}-3\right\}$. Это соответствие согласовано с полными порядками на множествах $\Omega^{n}$ и $\Psi^{n}$.

TеOPEMA 1.81. Пустъ $n \geqslant 1$. Тогда:

1) для любого множества $S \subseteq\{0,1, \ldots, n-1\}$ существует нетривиальное линейное соотношение, выражающее флаговое число $f_{S}(P)$ через флаговые числа $f_{S^{\prime}}(P), S^{\prime} \in \Psi^{n}$, которое выполнено для всех многогранников размерности $n$ (см. [17; предложение 2.2]);

2) флаговые векторы $\left\{f_{S}(Q)\right\}_{Q \in \Omega^{n}}$ порождают $\left(c_{n}-1\right)$-мерное афбинное пространство (см. [17; предложение 2.3]); 
3) флаговые векторы $\left\{f_{S^{\prime}}\left(P^{n}\right)\right\}_{S^{\prime} \in \Psi^{n}}$ порождают $\left(c_{n}-1\right)$-мерное афббинное пространство, определяемое уравнениями (26) и условием $f_{\varnothing}=1$ (см. [17; теорема 2.6]).

Первая часть теоремы следует из соотношений Байер-Биллера (26). Действительно, пусть $S \subseteq[0, n-1], S \notin \Psi^{n}$. Тогда для некоторого $k, 1 \leqslant k \leqslant n$, пара $\{k-1, k\}$ принадлежит $S \cup\{n\}$. Пусть $\widehat{S}=S \backslash\{k-1\}$ и $i=\max \{j \in$ $\{-1\} \cup S, j<k-1\}$. Запишем соотношение Байер-Биллера:

$$
f_{S}=\sum_{j=i+1}^{k-2}(-1)^{k-j} f_{\widehat{S} \cup\{j\}}+\left(1+(-1)^{k-i}\right) f_{\widehat{S}} .
$$

Все множества в правой части строго меньше множества $S$. Повторяя такое преобразование, в конце концов мы получим выражение числа $f_{S}$ через числа $\left\{f_{S^{\prime}}, S^{\prime} \in \Psi^{n}\right\}$ как линейную комбинацию с целыми коэффициентами, которое верно для всех $n$-мерных многогранников.

ЗАмЕЧАНИЕ 1.82. Здесь мы привели доказательство, следуя работе [17]. Из него видно, что коэффициенты линейного соотношения можно выбрать целыми числами и что все множества $\left\{S^{\prime} \in \Psi^{n}\right\}$, встречающиеся в выражении числа $f_{S}, S \notin \Psi^{n}$, можно выбрать строго меньшими, чем множество $S$.

Что касается второй части теоремы, то нам понадобится следующее более сильное утверждение.

УтВЕРЖДЕНИЕ 1.83. Рассмотрим матрицу

$$
K^{n}=\left\{k_{Q, S}=f_{S}(Q)\right\} \in \operatorname{Mat}_{c_{n} \times c_{n}}(\mathbb{Z}),
$$

где $n \geqslant 1, Q \in \Omega^{n}$ и $S \in \Psi^{n}$. Упорядочим строки и столбцы по возрастанию слов $Q$ и множеств $S$. Тогда $\operatorname{det} K^{n}=1$.

ДокАЗАТЕЛЬСтво. Для $n=1$ матрица $K^{1}$ имеет вид $\left(f_{\varnothing}\left(C^{2}\right)\right)=(1)$, где $C^{2}=I$.

Для $n=2$ имеем: $K^{2}=\left(\begin{array}{cc}1 & f_{0}\left(C^{3}\right) \\ 1 & f_{0}\left(B C^{2}\right)\end{array}\right)=\left(\begin{array}{ll}1 & 3 \\ 1 & 4\end{array}\right), \operatorname{det} K^{2}=1$, где $C^{3}=\Delta^{2}$ и $B C^{2}=I^{2}$.

Докажем утверждение индукцией по $n$. Пусть предположение верно для всех матриц $K^{r}, r<n$. Запишем матрицу $K^{n}$ в виде $\left(\begin{array}{ll}K_{11} & K_{12} \\ K_{21} & K_{22}\end{array}\right)$, где блок $K_{11}$ отвечает словам вида $C Q, Q \in \Omega^{n-1}$, и множествам $S$, которые не содержат элемент $n-2$, а блок $K_{22}$ отвечает словам вида $B C Q, Q \in \Omega^{n-2}$, и множествам, содержащим элемент $n-2$.

Грани многогранника $C P$ имеют вид либо $F \subseteq P$, либо $C F, \varnothing \subseteq F \subseteq P$, поэтому каждая возрастающая цепочка граней многогранника $C P^{n-1}$, не содержащая граней соседних размерностей, имеет вид

$$
F^{l_{1}} \subset \cdots \subset F^{l_{i}} \subset C F^{l_{i+1}} \subset \cdots \subset C F^{l_{k}},
$$

где

$$
F^{l_{1}} \subset \cdots \subset F^{l_{i}} \subset F^{l_{i+1}} \subset \cdots \subset F^{l_{k}} \subseteq P^{n-1},
$$


следовательно, для любого множества $S=\left\{a_{1}, \ldots, a_{k}\right\} \in \Psi^{n}$ мы имеем:

$$
\begin{aligned}
& f_{\left\{a_{1}, \ldots, a_{k}\right\}}\left(C P^{n-1}\right)=f_{\left\{a_{1}-1, \ldots, a_{k}-1\right\}}\left(P^{n-1}\right)+\cdots \\
& \quad+f_{\left\{a_{1}, \ldots, a_{i}, a_{i+1}-1, \ldots, a_{k}-1\right\}}\left(P^{n-1}\right)+\cdots+f_{\left\{a_{1}, \ldots, a_{k}\right\}}\left(P^{n-1}\right),
\end{aligned}
$$

где, как и раньше, $f_{\left\{-1, a_{2}, \ldots, a_{k}\right\}}=f_{\left\{a_{2}, \ldots, a_{k}\right\}}$.

Пусть $a_{k}<n-2$. Множества вида $\left\{a_{1}, \ldots, a_{l}, a_{l+1}-1, \ldots, a_{k}-1\right\}$ могут уже не принадлежать семейству $\Psi^{n-1}$. Однако, согласно первой части теоремы и замечанию 1.82 ,

$$
f_{\left\{a_{1}, \ldots, a_{k}\right\}}\left(C P^{n-1}\right)=f_{\left\{a_{1}, \ldots, a_{k}\right\}}\left(P^{n-1}\right)+\sum_{\substack{\widehat{S} \in \Psi^{n-1} \\ \widehat{S}<\left\{a_{1}, \ldots, a_{k}\right\}}} \lambda_{\widehat{S}} f_{\widehat{S}}\left(P^{n-1}\right), \quad \lambda_{\widehat{S}} \in \mathbb{Z} .
$$

Поэтому матрицу $K_{11}$ можно представить в виде $K^{n-1} R$, где $R$ - верхняя унитреугольная матрица. В частности, $\operatorname{det} K_{11}=\operatorname{det} K^{n-1} \cdot \operatorname{det} R=1$ по предположению индукции.

Лемма 1.84. Пусть $P-(n-2)$-мерный многогранник и $S \in \Psi^{n}$. Тогда

$$
f_{S}(B C P)= \begin{cases}f_{S}(C B P), & \text { если } n-2 \notin S, \\ f_{S}(C B P)+f_{S \backslash\{n-2\}}(P), & \text { если } n-2 \in S .\end{cases}
$$

ДокАЗАТЕЛьство. Лемма является прямым следствием утверждения 1.76.

Теперь рассмотрим многогранник $B C Q$, отвечающий одной из нижних $c_{n-2}$ строк матрицы $K^{n}$. Если слово $Q$ начинается с символа $C$, то ему соответствует слово $C B Q$ и строка в верхней части матрицы. Вычтем эту строку из строки, которой отвечает $B C Q$. Согласно лемме 1.84 , мы получим

$$
k_{B C Q, S}^{\prime}= \begin{cases}0, & \text { если } n-2 \notin S ; \\ f_{S \backslash\{n-2\}}(Q), & \text { если } n-2 \in S .\end{cases}
$$

Если же слово $Q$ начинается с символа $B$, то ему не соответствует никакая строка в верхней части матрицы. Однако по предположению индукции $\operatorname{det} K^{n-1}=1$. Кроме того, все флаговые числа $(n-1)$-мерных многогранников выражаются с целыми коэффициентами через $c_{n-1}$ чисел $\left\{f_{S}, S \in \Psi^{n-1}\right\}$. Поэтому флаговый вектор любого $(n-1)$-мерного многогранника $P^{n-1}$ является линейной комбинацией с целыми коэффициентами флаговых векторов многогранников из множества $\Omega^{n-1}$. В частности, $f_{S}(B Q)=\sum_{Q^{\prime} \in \Omega^{n-1}} n_{Q^{\prime}} f_{S}\left(Q^{\prime}\right), S \subseteq[0, n-2]$. Используя формулу (28), мы получаем $f_{S}(C B Q)=\sum_{Q^{\prime} \in \Omega^{n-1}} n_{Q^{\prime}} f_{S}\left(C Q^{\prime}\right), S \in \Psi^{n}$. Вычитая соответствующую линейную комбинацию строк из верхней части матрицы, мы снова получаем формулу (30).

Таким образом, элементарными преобразованиями строк матрица $K^{n}$ может быть приведена к виду

$$
\left(\begin{array}{cc}
K^{n-1} R & K_{12} \\
0 & K^{n-2}
\end{array}\right)
$$

Используя предположение индукции, получаем $\operatorname{det} K^{n}=1$. 
ЗАмечАниЕ 1.85. Доказательство утверждения 1.83 можно получить следуя работе [17]. Разница в наших доказательствах заключается в том, что М. Байер и Л. Биллера переходят к новой матрице чисел граней многогранников $Q \in \Psi^{n}$, а мы работаем непосредственно с флаговыми числами, пользуясь формулой (28) и леммой 1.84. В [17] не отмечен важный для нас факт, что $\operatorname{det} K_{n}=1$.

СЛЕДСТВИЕ 1.86. Любой целочисленный вектор $\left\{g_{S}, S \subseteq[0, n-1]\right\}$, удовлетворяющий соотношениям (26), однозначно представляется в виде целочисленной линейной комбинации векторов $\left\{f_{S}(Q), S \subseteq[0, n-1]\right\}, Q \in \Psi^{n}$. $B$ частности, это верно для флагового вектора $n$-мерного многогранника.

ДокАзАтельство. Любая координата $g_{S}$ вектора $\left\{g_{S}, S \subseteq[0, n-1]\right\}$, удовлетворяющего соотношениям (26), выражается через числа $\left\{g_{S^{\prime}}, S^{\prime} \in \Psi^{n}\right\}$ по той же формуле, по которой флаговое число $f_{S}$ любого $n$-мерного многогранника $P^{n}$ выражается через флаговые числа $\left\{f_{S^{\prime}}, S^{\prime} \in \Psi^{n}\right\}$. Так как $\operatorname{det} K^{n}=1$, то любой вектор $g_{\Psi^{n}}=\left\{g_{S^{\prime}}, S^{\prime} \in \Psi^{n}\right\}$ единственным образом представляется в виде целочисленной линейной комбинации векторов $f_{\Psi^{n}}(Q), Q \in \Omega^{n}$. Следовательно, и вектор $\left\{g_{S}, S \subseteq[0, n-1]\right\}$ однозначно представляется в виде целочисленной линейной комбинации флаговых векторов многогранников $Q \in \Omega^{n}$ с теми же коэффициентами.

Следствие будет использовано при исследовании образа флаговых полиномов в кольце $\mathcal{Q} \operatorname{sym}[\alpha]$ (см. ниже).

О связи флагового вектора и $\boldsymbol{c d}$-индекса см. приложение Е.

ОПРЕДЕЛЕНИЕ 1.87. Будем говорить, что множество $T^{2 n}$ из $c_{n}$ элементов группы $\mathcal{P}^{2 n}=\mathcal{R} \mathcal{P}^{2(n+1)}, n \geqslant 0$, задает базис в пространстве флаговых векторов, если определитель матрицы $K_{T}^{n}=\left\{k_{P, S}=f_{S}(P)\right\} \in \operatorname{Mat}_{c_{n} \times c_{n}}(\mathbb{Z})$, где $P \in T^{2 n}$ и $S \in \Psi^{n}$, равен \pm 1 .

УтВЕРЖДЕНИЕ 1.88. Пусть множества $T^{2(n-1)}$ u $T^{2(n-2)}, n \geqslant 2$, задают базисы в пространствах флаговых векторов. Тогда множество $T^{2 n}=$ $\left(\mathscr{A} T^{2(n-1)}\right) \cup\left(\mathscr{D} T^{2(n-2)}\right)$ задает базис в пространстве флаговых векторов.

Доказательство. Упорядочим множества $S \in \Psi^{n}$ по возрастанию. Матрицу $K_{T}^{n}$ можно представить в виде $\left(\begin{array}{ll}K_{11} & K_{12} \\ K_{21} & K_{22}\end{array}\right)$, где блок $K_{11}$ отвечает элементам вида $\mathscr{A} P, P \in T^{2(n-1)}$, и множествам $S$, которые не содержат элемент $n-2$, а блок $K_{22}$ отвечает словам вида $\mathscr{D} P, P \in T^{2(n-2)}$, и множествам, содержащим элемент $n-2$. Согласно утверждению 1.76 , имеем: $K_{11}=K_{T}^{n-1}$, $K_{21}=0$ и $K_{22}=K_{T}^{n-2}$. Поэтому $\operatorname{det} K_{T}^{n}=\operatorname{det} K_{T}^{n-1} \cdot \operatorname{det} K_{T}^{n-2}= \pm 1$.

Обозначим через $T_{\mathscr{A} \mathscr{D}}^{2 n} \subset \mathcal{P}^{2 n}, n \geqslant 0$, множество, состоящее из элементов, получающихся из точки рt применением всевозможных слов степени $2 n$ из букв $\mathscr{A}$ и $\mathscr{D}$, где $\operatorname{deg} \mathscr{A}=2$ и $\operatorname{deg} \mathscr{D}=4$.

СлЕДСТВИЕ 1.89. Для любого $n \geqslant 0$ множество $T_{\mathscr{A} \mathscr{D}}^{2 n}$ задает базис в пространстве флаговых векторов. 
ПРИМЕР 1.90. Имеем:

$$
\begin{aligned}
& T_{\mathscr{A} \mathscr{D}}^{0}=\{\mathrm{pt}\}, \quad T_{\mathscr{A} \mathscr{D}}^{2}=\{\mathscr{A} \mathrm{pt}=I\}, \quad T_{\mathscr{A} \mathscr{D}}^{4}=\left\{\mathscr{A}^{2} \mathrm{pt}=2 \Delta^{2}-I^{2}, \mathscr{D} \mathrm{pt}=I^{2}-\Delta^{2}\right\}, \\
& T_{\mathscr{A} \mathscr{D}}^{6}=\left\{\mathscr{A}^{3} \mathrm{pt}=4 \Delta^{3}-2 C I^{2}-2 B \Delta^{2}+B I^{2},\right. \\
&\left.\mathscr{A} \mathscr{D} \mathrm{pt}=-2 \Delta^{3}+2 C I^{2}+B \Delta^{2}-B I^{2}, \mathscr{D} \mathscr{A} \mathrm{pt}=B \Delta^{2}-C I^{2}\right\} .
\end{aligned}
$$

ОПРедЕЛЕНИЕ 1.91. Пусть $\mathcal{P} \mathscr{A} \mathscr{D}$ - абелева подгруппа в $\mathcal{P}$, порожденная всеми элементами множеств $T_{\mathscr{A} \mathscr{D}}^{2 n}, n \geqslant 0$. Определим проекцию $\pi_{\mathscr{A} \mathscr{D}}: \mathcal{P} \rightarrow \mathcal{P}_{\mathscr{A} \mathscr{D}}$, которая сопоставляет элементу $P \in \mathcal{P}^{2 n}$ элемент $\pi_{\mathscr{A} \mathscr{D}}(P) \in \mathcal{P}_{\mathscr{A} \mathscr{D}}^{2 n}$, который имеет такой же флаговый вектор. Пусть $P \in \mathcal{P}^{2 n}$. Тогда

$$
\pi_{\mathscr{A} \mathscr{D}}(P)=\sum[W]_{P}(W \mathrm{pt})
$$

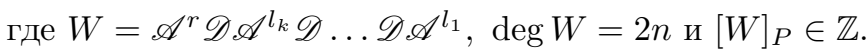

ОПРЕДЕЛЕНиЕ 1.92. Рассмотрим правое действие свободной ассоциативной алгебры $\mathcal{Q}=\mathbb{Z}\langle\boldsymbol{c}, \boldsymbol{d}\rangle, \operatorname{deg} \boldsymbol{c}=2, \operatorname{deg} \boldsymbol{d}=4$, на кольце $\mathcal{P}$, при котором $P \boldsymbol{c}=\mathscr{A} P$ и $P \boldsymbol{d}=\mathscr{D} P$.

Имеем: $1 \mathbb{Z}\langle\boldsymbol{c}, \boldsymbol{d}\rangle=\mathcal{P}_{\mathscr{A} \mathscr{D}}$, поэтому отображение $\mathcal{Q} \rightarrow \mathbb{Z}\langle\mathscr{A}, \mathscr{D}\rangle$ является антиизоморфизмом.

ОПРЕДЕЛЕНИЕ 1.93. Пусть $T$ - градуированное частично упорядоченное множество ранга $n+1$. Для множества $S=\left\{a_{1}, \ldots, a_{k}\right\} \subseteq\{0,1,2, \ldots, n-1\}$ определим флаговое число $f_{S}(T)$ как число возрастающих цепочек элементов $x_{1}<\cdots<x_{k}, \varrho\left(x_{i}\right)=a_{i}+1$.

Из конструкций множеств $T_{1}$ и $T_{2}$ (см. определение 1.35 ) легко следует следующий результат.

УТВЕРЖДЕНИЕ 1.94. Пусть $T \in \mathcal{R}^{2 n}$ - частично упорядоченное множество и $S \subseteq\{0,1, \ldots, n-1\}$. Тогда

$$
f_{S}\left(T_{1}\right)=\left\{\begin{array}{ll}
f_{S}(T), & n-1 \notin S, \\
f_{S \backslash\{n-1\}}(T), & n-1 \in S,
\end{array} \quad f_{S}\left(T_{2}\right)= \begin{cases}f_{S}(T), & n-1 \notin S, \\
2 f_{S \backslash\{n-1\}}(T), & n-1 \in S,\end{cases}\right.
$$

где для $n=0$ ми считаем, что $-1 \in S=\varnothing$.

СлЕДСТВие 1.95. Для операторов $\mathscr{O}_{0}$ и $\mathscr{O}_{1}$ имеем:

$$
f_{S}\left(\mathscr{O}_{0} T\right)=\left\{\begin{array}{ll}
f_{S}(T), & n-1 \notin S, \\
0, & n-1 \in S,
\end{array} \quad f_{S}\left(\mathscr{O}_{1} T\right)= \begin{cases}0, & n-1 \notin S, \\
f_{S \backslash\{n-1\}}(T), & n-1 \in S .\end{cases}\right.
$$

Для флаговых чисел многогранников из кольца $\mathcal{R} \mathcal{P}$ существует каноническая операция умножения. См. приложение В.

\section{2. Квазисимметрические функции и алгебры Хопфа}

Необходимые сведения из теории алгебр Хопфа можно найти в [18], [19]. В этом разделе мы следуем, преимущественно, обозначениям работы [20]. См. также [21] и [22].

Пусть $R$ - кольцо и $T$ - конечное или счетное множество. Через $R\langle T\rangle$ и $R[T]$ мы будем обозначать соответственно свободную ассоциативную алгебру и алгебру полиномов над $R$ от образующих $T$. 
2.1. Определения и конструкции. Теория квазисимметрических функций возникла и развилась за последние двадцать лет под влиянием проблем из различных направлений исследований. В настоящее время она широко известна, богата результатами и приложениями. См. [13], [14], [20], [21], [23]-[29].

ОПРЕДЕЛЕНИЕ 2.1. Упорядоченное множество $\omega=\left(j_{1}, \ldots, j_{k}\right), j_{i} \geqslant 1$, где $j_{1}+\cdots+j_{k}=n$, называется композицией числа $n$. Положим $|\omega|=n$ и $l(\omega)=k$.

Пустую композицию числа 0 мы будем обозначать символом (). Имеем $|()|=0, l(())=0$.

Определим на множестве композиций числа $n$ частичный порядок, задав накрывающие отношения $\left(j_{1}, \ldots, j_{i}+j_{i+1}, \ldots, j_{k}\right) \prec\left(j_{1}, \ldots, j_{i}, j_{i+1}, \ldots, j_{k}\right)$. В этом случае максимальной будет композиция $(1,1, \ldots, 1)$, а минимальной $-(n)$.

Для композиции $\omega=\left(j_{1}, \ldots, j_{k}\right)$ положим $\omega^{*}=\left(j_{k}, \ldots, j_{1}\right)$.

Пусть $n \geqslant 1$. Для множества $S=\left\{a_{1}, \ldots, a_{k}\right\} \subseteq\{0,1, \ldots, n-1\}$ обозначим через $\omega(S)$ композицию $\left(n-a_{k}, a_{k}-a_{k-1}, \ldots, a_{2}-a_{1}\right)$ числа $n-a_{1}$, а для композиции $\omega=\left(j_{1}, \ldots, j_{k}\right)$ положим $S(\omega)=\left\{n-|\omega|, n-|\omega|+j_{k}, \ldots, n-j_{1}\right\}$.

ОПРЕДЕЛЕНИЕ 2.2. Пусть $t_{1}, t_{2}, \ldots$ - конечное или счетное множество переменных степени $\operatorname{deg} t_{i}=2$. Квазисимметрическим мономом $M_{\omega}$ композиции $\omega=\left(j_{1}, \ldots, j_{k}\right)$ называется выражение $M_{\omega}=\sum_{l_{1}<\cdots<l_{k}} t_{l_{1}}^{j_{1}} \cdots t_{l_{k}}^{j_{k}}, M_{()}=1$.

Степень монома $M_{\omega}$ равна $2|\omega|=2\left(j_{1}+\cdots+j_{k}\right)$. Для любых двух мономов $M_{\omega}$ и $M_{\omega^{\prime}}$ их произведение в кольце полиномов $\mathbb{Z}\left[t_{1}, t_{2}, \ldots\right]$ равно

$$
M_{\omega^{\prime}} M_{\omega^{\prime \prime}}=\sum_{\omega}\left(\sum_{\Omega^{\prime}+\Omega^{\prime \prime}=\omega} 1\right) M_{\omega}
$$

где для композиций $\omega=\left(j_{1}, \ldots, j_{k}\right), \omega^{\prime}=\left(j_{1}^{\prime}, \ldots, j_{l^{\prime}}^{\prime}\right)$ и $\omega^{\prime \prime}=\left(j_{1}^{\prime \prime}, \ldots, j_{l^{\prime \prime}}^{\prime \prime}\right)$ через $\Omega^{\prime}$ и $\Omega^{\prime \prime}$ обозначены всевозможные строки длины $k$ такие, что

$$
\Omega^{\prime}=\left(0, \ldots, j_{1}^{\prime}, \ldots, 0, \ldots, j_{l^{\prime}}^{\prime}, \ldots, 0\right), \quad \Omega^{\prime \prime}=\left(0, \ldots, j_{1}^{\prime \prime}, \ldots, 0, \ldots, j_{l^{\prime \prime}}^{\prime \prime}, \ldots, 0\right) .
$$

Правило умножения композиций называется перекрывающимся тасовочным умножением. Например,

$$
M_{(1)} M_{(1)}=\left(\sum_{i} t_{i}\right)\left(\sum_{j} t_{j}\right)=\sum_{i} t_{i}^{2}+2 \sum_{i<j} t_{i} t_{j}=M_{(2)}+2 M_{(1,1)},
$$

что соответствует разложениям

$$
(2)=(1)+(1), \quad(1,1)=(1,0)+(0,1)=(0,1)+(1,0),
$$

и

$$
\begin{aligned}
M_{(1)} M_{(1,1)} & =\left(\sum_{i} t_{i}\right)\left(\sum_{j<k} t_{j} t_{k}\right)=\sum_{i<j} t_{i}^{2} t_{j}+\sum_{i<j} t_{i} t_{j}^{2}+3 \sum_{i<j<k} t_{i} t_{j} t_{k} \\
& =M_{(2,1)}+M_{(1,2)}+3 M_{(1,1,1)}
\end{aligned}
$$

что соответствует разложениям

$$
\begin{gathered}
(2,1)=(1,0)+(1,1), \quad(1,2)=(0,1)+(1,1), \\
(1,1,1)=(1,0,0)+(0,1,1)=(0,1,0)+(1,0,1)=(0,0,1)+(1,1,0) .
\end{gathered}
$$


Таким образом, конечные линейные комбинации квазисимметрических мономов образуют градуированное кольцо. Это кольцо называется кольцом квазисимметрических функций и обозначается

в случае $n$ переменных, либо

$$
\operatorname{Qsym}\left[t_{1}, \ldots, t_{r}\right]=\sum_{n \geqslant 0} \operatorname{Qsym}^{2 n}\left[t_{1}, \ldots, t_{r}\right]
$$

$$
\operatorname{Qsym}\left[t_{1}, t_{2}, \ldots\right]=\sum_{n \geqslant 0} \operatorname{Qsym}^{2 n}\left[t_{1}, t_{2}, \ldots\right] \quad \text { или } \quad \mathcal{Q} \text { sym }=\sum_{n \geqslant 0} \mathcal{Q} \operatorname{sym}^{2 n}
$$

в случае бесконечного числа переменных, где группы Qsym ${ }^{2 n}\left[t_{1}, \ldots, t_{r}\right]$ и $\mathcal{Q}_{s y m}{ }^{2 n}$ состоят из однородных квазисимметрических функций степени $2 n$.

ЗАмЕчАНИЕ 2.3. Мы будем часто пользоваться изоморфностью ограничения $\mathcal{Q}_{\text {sym }^{2 n}} \rightarrow \operatorname{Qsym}^{2 n}\left[t_{1}, \ldots, t_{r}\right]$ при $r \geqslant n$.

ОПРЕДЕЛЕНИЕ 2.4. Соответствие $M_{\omega} \rightarrow M_{\omega^{*}}$ задает кольцевой гомоморфизм $*: \mathcal{Q}$ sym $\rightarrow \mathcal{Q}$ sym, который является инволюцией.

УтвеРЖДЕНИЕ 2.5. Полином $g \in \mathbb{Z}\left[t_{1}, \ldots, t_{r}\right]$ принадлежит Qsym $\left[t_{1}, \ldots, t_{r}\right]$ тогда и толъко тогда, когда

$$
g\left(0, t_{1}, t_{2}, \ldots, t_{r-1}\right)=g\left(t_{1}, 0, t_{2}, \ldots, t_{r-1}\right)=\cdots=g\left(t_{1}, \ldots, t_{r-1}, 0\right) .
$$

Полином $g\left(t_{1}, t_{2}, \ldots\right)$ вида

$$
a+\sum_{k=1}^{\infty} \sum_{1 \leqslant l_{1}<\cdots<l_{k}} \sum_{j_{1}, \ldots, j_{k} \geqslant 1} a_{l_{1}, \ldots, l_{k}}^{j_{1}, \ldots, j_{k}} t_{l_{1}}^{j_{1}} \cdots t_{l_{k}}^{j_{k}}
$$

степени $2\left(j_{1}+\cdots+j_{k}\right) \leqslant N$ принадлежит $\mathcal{Q}$ sут тогда и только тогда, когда

$$
g\left(t_{1}, \ldots, t_{i-1}, 0, t_{i}, \ldots\right)=g\left(t_{1}, \ldots, t_{i-1}, t_{i}, \ldots\right) \quad \text { для всех } i \geqslant 1 .
$$

ДокАЗАТЕЛЬство. Для квазисимметрического монома $M_{\omega}$ мы имеем:

$$
M_{\omega}\left(t_{1}, \ldots, t_{i}, 0, t_{i+1}, \ldots, t_{r-1}\right)= \begin{cases}0, & l(\omega)=r \\ M_{\omega}\left(t_{1}, \ldots, t_{i}, t_{i+1}, \ldots, t_{r-1}\right), & l(\omega)<r\end{cases}
$$

в случае $r$ переменных, и $M_{\omega}\left(t_{1}, \ldots, t_{i-1}, 0, t_{i}, \ldots\right)=M_{\omega}\left(t_{1}, \ldots, t_{i-1}, t_{i}, \ldots\right)$ в случае бесконечного числа переменных, поэтому для квазисимметрических функций условия (31) и (33) выполнены.

С другой стороны, докажем, что выполнение условия (31) или (33) влечет равенство любых двух коэффициентов вида $a_{l_{1}, \ldots, l_{k}}^{j_{1}, \ldots, j_{k}}$ и $a_{l_{1}^{\prime}, \ldots, l_{k}^{\prime}}^{j_{1}, \ldots, j_{k}}$.

Сначала заметим, что из условия (33) следуют равенства

$$
g\left(0, t_{1}, t_{2}, t_{3}, \ldots\right)=g\left(t_{1}, 0, t_{2}, t_{3}, \ldots\right)=g\left(t_{1}, t_{2}, 0, t_{3} \cdots\right)=\cdots .
$$

Пусть $l_{i}>l_{i-1}+1$ или $i=1, l_{i}>1$. Рассмотрим соответствующие коэффициенты в уравнении $g\left(t_{1}, \ldots, t_{l_{i}-2}, 0, t_{l_{i}-1}, t_{l_{i}}, \ldots\right)=g\left(t_{1}, \ldots, t_{l_{i}-1}, 0, t_{l_{i}}, \ldots\right)$. Слева моном $t_{l_{1}}^{j_{1}} \cdots t_{l_{i-1}}^{j_{i-1}} t_{l_{i}-1}^{j_{i}} t_{l_{i+1}-1}^{j_{i+1}} \cdots t_{l_{k}-1}^{j_{k}}$ имеет коэффициент $a_{l_{1}, \ldots, l_{i-1}, l_{i}, l_{i+1}, \ldots, l_{k}}^{j_{1}, \ldots, j_{i-1}, j_{i}, j_{i+1}, \ldots, j_{k}}$, а справа $-a_{l_{1}, \ldots, l_{i-1}, l_{i}-1, l_{i+1}, \ldots, l_{k}}^{j_{1}, \ldots,,_{j-1}, j_{i}, j_{i+1}, \ldots, j_{k}}$, поэтому они равны. Следовательно, мы можем "передвинуть" индекс $l_{i}$ на 1 влево.

Теперь для любого коэффициента $a_{l_{1}, \ldots, l_{k}}^{j_{1}, \ldots, j_{k}}$ мы можем шаг за шагом передвинуть индекс $l_{1}$ в 1 , индекс $l_{2}-$ в $2, \ldots$, индекс $l_{k}-$ в $k$. Таким образом, $a_{l_{1}, \ldots, l_{k}}^{j_{1}, \ldots, j_{k}}=a_{1, \ldots, k}^{j_{1}, \ldots, j_{k}}$. 
Следовательно, полином $g$ является линейной комбинацией квазисимметрических мономов. Так как его степень ограничена, то число мономов конечно, поэтому $g$ является квазисимметрической функцией. Утверждение доказано.

В качестве следствия мы получаем другое доказательство того, что квазисимметрические функции образуют кольцо.

М. Хазевинкель в [20] доказал гипотезу Диттерса о том, что кольцо $\mathcal{Q} s y m$ является алгеброй полиномов над кольцом целых чисел.

Tак как $\operatorname{rk} \mathcal{Q} \operatorname{sym}^{2 n}=2^{n-1}$, то числа $\beta_{i}$ мультипликативных образующих степени $2 i$ могут быть найдены из рекуррентного соотношения

$$
\frac{1-t}{1-2 t}=\prod_{i=1}^{\infty} \frac{1}{\left(1-t^{i}\right)^{\beta_{i}}} .
$$

Определены гомоморфизмы абелевых групп:

$$
\begin{aligned}
& V_{r+1}: \operatorname{Qsym}\left[t_{1}, \ldots, t_{r}\right] \rightarrow \operatorname{Qsym}\left[t_{1}, \ldots, t_{r+1}\right], \\
& V_{r+1} M_{\omega}\left(t_{1}, \ldots, t_{r}\right)=M_{\omega}\left(t_{1}, \ldots, t_{r+1}\right) ; \\
& E_{r}: \operatorname{Qsym}\left[t_{1}, \ldots, t_{r+1}\right] \rightarrow \operatorname{Qsym}\left[t_{1}, \ldots, t_{r}\right], \quad t_{r+1} \rightarrow 0 .
\end{aligned}
$$

Отображение $V_{r+1}$ мультипликативно для мономов $M_{\omega_{1}}$ и $M_{\omega_{2}}$ таких, что $l\left(\omega_{1}\right)+l\left(\omega_{2}\right) \leqslant r$. Отображение $E_{r}$ переводит мономы $M_{\omega}, l(\omega)=r+1$, в 0 , в то время как для композиции $\omega, l(\omega)<r+1$, имеем $E_{r} M_{\omega}\left(t_{1}, \ldots, t_{r}, t_{r+1}\right)=$ $M_{\omega}\left(t_{1}, \ldots, t_{r}\right)$. Оно является кольцевым гомоморфизмом.

Легко видеть, что $E_{r} V_{r+1}$ - тождественное отображение.

Для $r>0$ определен оператор $\Pi_{\mathrm{Qsym}}: \mathbb{Z}\left[t_{1}, \ldots, t_{r}\right] \rightarrow \operatorname{Qsym}\left[t_{1}, \ldots, t_{r}\right] \otimes \mathbb{Q}:$

$$
\Pi_{\mathrm{Qsym}} t_{s_{1}}^{j_{1}} \cdots t_{s_{k}}^{j_{k}}=\frac{1}{\left(\begin{array}{c}
r \\
k
\end{array}\right)}\left(\sum_{l_{1}<\cdots<l_{k}} t_{l_{1}}^{j_{1}} \cdots t_{l_{k}}^{j_{k}}\right), \quad s_{1}<\cdots<s_{k},
$$

сопоставляющий моному среднее значение по всем мономам заданного типа.

Для квазисимметрического монома $M_{\omega}, \omega=\left(j_{1}, \ldots, j_{k}\right)$, мы имеем:

$$
\Pi_{\mathrm{Qsym}} M_{\omega}=\sum_{1 \leqslant l_{1}<\cdots<l_{k} \leqslant r} \frac{1}{\left(\begin{array}{c}
r \\
k
\end{array}\right)} M_{\omega}=\left(\begin{array}{l}
r \\
k
\end{array}\right) \frac{1}{\left(\begin{array}{l}
r \\
k
\end{array}\right)} M_{\omega}=M_{\omega},
$$

поэтому оператор $\Pi_{\mathrm{Qsym}}$ является проектором.

ЗАмЕчАНиЕ 2.6. Мы видим, что в теории симметрических и квазисимметрических функций важную роль играет такая характеристика, как число переменных в полиноме.

УтВЕРЖДЕНИЕ 2.7. Колъцо квазисимметрических функиий $\mathcal{Q} s y m$ имеет структуру алгебры Хопфа:

- умножением $\mu: \mathcal{Q}$ sym $\otimes \mathcal{Q}$ sym $\rightarrow \mathcal{Q}$ sym служит перекрывающееся тасовочное умножение;

- единица $\imath: \mathbb{Z} \rightarrow \mathcal{Q}$ sуm переводит единичу $1 \in \mathbb{Z}$ в моном $M_{(\text {) }}$;

- коумножение $\Delta: \mathcal{Q}$ sym $\rightarrow \mathcal{Q}$ sym $\otimes \mathcal{Q}$ sym задается формулой

$$
\Delta\left(M_{\left(j_{1}, \ldots, j_{k}\right)}\right)=\sum_{i=0}^{k} M_{\left(j_{1}, \ldots, j_{i}\right)} \otimes M_{\left(j_{i+1}, \ldots, j_{k}\right)} ;
$$


- коединица $\varepsilon: \mathcal{Q}$ sуm $\rightarrow \mathbb{Z}$ задается формулой

$$
\varepsilon\left(M_{\omega}\right)= \begin{cases}1, & \text { если } \omega=(), \\ 0 & \text { в противном случае; }\end{cases}
$$

- антипод $\chi: \mathcal{Q}$ sym $\rightarrow \mathcal{Q}$ sym действует по формуле (см. [13], [26])

$$
\chi\left(M_{\omega}\right)=(-1)^{l(\omega)} \sum_{\sigma \leqslant \omega} M_{\sigma^{*}},
$$

где суммирование ведется по всем композициям $\sigma, \sigma \leqslant \omega$, числа $|\omega|$.

Мы имеем $\Delta\left(M_{\omega}^{*}\right)=* \otimes *\left(\tau_{\mathcal{Q} s y m, \mathcal{Q} s y m} \Delta M_{\omega}\right)$, т. е. инволюция $*$ задает изоморфизм алгебр Хопфа $\mathcal{Q}$ sym и $\mathcal{Q} s y m{ }^{\text {op cop }}$.

ОПРЕДЕЛЕНИЕ 2.8. Определим линейные операторы $\mathscr{O}_{0}, \mathscr{O}_{1}: \mathcal{Q} s y m \rightarrow \mathcal{Q} s y m$ формулами

$$
\begin{array}{ll}
\mathscr{O}_{0} M_{\left(j_{1}, j_{2}, \ldots, j_{k-1}, j_{k}\right)}=M_{\left(j_{1}, j_{2}, \ldots, j_{k-1}, j_{k}+1\right)}, & \mathscr{O}_{0} M_{()}=0 ; \\
\mathscr{O}_{1} M_{\left(j_{1}, j_{2}, \ldots, j_{k-1}, j_{k}\right)}=M_{\left(j_{1}, j_{2}, \ldots, j_{k-1}, j_{k}, 1\right)}, & \mathscr{O}_{1} M_{()}=M_{(1)} .
\end{array}
$$

УТВЕРЖДЕНИЕ 2.9. $\mathcal{Q}$ sym $=\mathbb{Z}\left\langle\mathscr{O}_{0}, \mathscr{O}_{1}\right\rangle M_{()}$, причем имеет место изоморфизм абелевых групп $\mathbb{Z}\left\langle\mathscr{O}_{0}, \mathscr{O}_{1}\right\rangle \simeq \mathbb{Z}\left\langle\mathscr{O}_{0}, \mathscr{O}_{1}\right\rangle M_{(1)}$

ДоказАтельСтво. Имеем $M_{(1)}=\mathscr{O}_{1} M_{()}, M_{\left(j_{1}, \ldots, j_{k-1}, 1\right)}=\mathscr{O}_{1} M_{\left(j_{1}, \ldots, j_{k-1}\right)}$ и $M_{\left(j_{1}, \ldots, j_{k}\right)}=\mathscr{O}_{0} M_{\left(j_{1}, \ldots, j_{k}-1\right)}$, если $j_{k}>1$. Поэтому все квазисимметрические мономы принадлежат группе $\mathbb{Z}\left\langle\mathscr{O}_{0}, \mathscr{O}_{1}\right\rangle M_{()}$. При этом соответствие $W \rightarrow$ $W M_{(1)}$ задает изоморфизм абелевых групп $\mathbb{Z}\left\langle\mathscr{O}_{0}, \mathscr{O}_{1}\right\rangle \simeq \mathbb{Z}\left\langle\mathscr{O}_{0}, \mathscr{O}_{1}\right\rangle M_{(1)}$.

ОПРЕДЕЛЕНИЕ 2.10. Пусть $\psi: R_{1} \rightarrow R_{2}$ - гомоморфизм колец. Отображение $A: R_{1} \rightarrow R_{2}[\alpha]$ называется $\psi$ - $\alpha$-дифференцированием, если для любых $a, b \in R_{1}$

$$
A(a b)=A(a) \psi(b)+\psi(a) A(b)+\alpha A(a) A(b) .
$$

ПримеР 2.11. Следующие утверждения проверяются непосредственно.

(i) Отображение $A_{1}: \mathbb{Z}[x] \rightarrow \mathbb{Z}[x, \alpha]$, переводящее полином $f(x)$ в разностную производную $(f(x+\alpha)-f(x)) / \alpha$, является id- $\alpha$-дифференцированием.

(ii) Отображение $A_{2}: \mathbb{Z}[x, y] \rightarrow \mathbb{Z}[x, y], f(x, y) \rightarrow(f(x, y)-f(y, x)) /(x-y)$, является id- $\alpha$-дифференцированием, где $\alpha=x-y$. Заметим, что

$$
A_{2}(f)=\frac{f(y+\alpha, y)-f(y, y)}{\alpha}-\frac{f(y, y+\alpha)-f(y, y)}{\alpha} .
$$

(iii) Отображение $\delta: \mathcal{P} \rightarrow \mathbb{Z}[\alpha, t], \delta(\alpha, t)(P)=f(\alpha, t)(d P)-\frac{\partial}{\partial t} f(\alpha, t)(P)$, является $f$-0-дифференцированием.

Развитие и приложения теории $\psi$ - $\alpha$-дифференцирований можно найти в [30].

ОПредЕЛЕниЕ 2.12. Введем отображение $\partial: \mathcal{Q} s y m \rightarrow \mathcal{Q} s y m[\alpha]$ формулой $\partial M_{\left(j_{1}, \ldots, j_{k}\right)}=\alpha^{j_{1}-1} M_{\left(j_{2}, \ldots, j_{k}\right)}, \partial M_{()}=0$.

Следующий результат получается непосредственной проверкой.

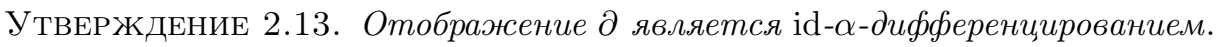




\section{2. Отображение Эренборга.}

ОПРЕДЕЛЕНИЕ 2.14. Пусть $T \in \mathcal{R}^{2 n}$ - частично упорядоченное множество. Определим квазисимметрическую функцию

$$
\begin{aligned}
& \mathrm{F}(T)=\sum_{\hat{0}=x_{0}<x_{1}<\cdots<x_{k+1}=\hat{1}} M_{\left(\rho\left(x_{0}, x_{1}\right), \rho\left(x_{1}, x_{2}\right), \ldots, \rho\left(x_{k}, x_{k+1}\right)\right)} \\
& =\sum_{\left\{a_{1}, \ldots, a_{k}\right\} \in 2^{[0, n-2]}} f_{\left\{a_{1}, \ldots, a_{k}\right\}} M_{\left(a_{1}+1, a_{2}-a_{1}, \ldots, n-1-a_{k}\right)},
\end{aligned}
$$

где первая сумма берется по всем возрастающим цепочкам элементов. Имеем: $\mathrm{F}(1)=1$ и $\mathrm{F}(\{\hat{0}, \hat{1}\})=M_{(1)}$. Соответствие $T \rightarrow F(T)$ задает отображение Эренборга $\mathrm{F}: \mathcal{R} \rightarrow \mathcal{Q}$ sym.

Отображение Эренборга является гомоморфизмом алгебр Хопфа, причем $\mathrm{F}\left(T^{*}\right)=\mathrm{F}(T)^{*}$ (см. [13]). Пусть $\mathscr{O}_{i}$ - операторы из определения 1.35 .

УтвеРЖДЕниЕ 2.15. Имеем: $\mathrm{F}\left(\mathscr{O}_{i} T\right)=\mathscr{O}_{i} \mathrm{~F}(T)$ для $i=1,2$ u $T \in \mathcal{R}$.

Доказательство. Пусть $T \in \mathcal{R}^{2 n}$. Имеем: $\mathrm{F}\left(\mathscr{O}_{0} 1\right)=\mathrm{F}(0)=0=\mathscr{O}_{0} M_{()}$, $\mathrm{F}\left(\mathscr{O}_{1} 1\right)=\mathrm{F}(\{\hat{0}, \hat{1}\})=M_{(1)}=\mathscr{O}_{1} M_{()}$. Пользуясь следствием 1.95 , для $n \geqslant 1$ получаем:

$$
\begin{aligned}
\mathrm{F}\left(\mathscr{O}_{0} T\right) & =\sum_{\left\{a_{1}, \ldots, a_{k}\right\} \in 2^{[0, n-1]}} f_{\left\{a_{1}, \ldots, a_{k}\right\}}\left(\mathscr{O}_{0} T\right) M_{\left(a_{1}+1, a_{2}-a_{1}, \ldots, n-a_{k}\right)} \\
= & \sum_{\left\{a_{1}, \ldots, a_{k}\right\} \in 2^{[0, n-2]}} f_{\left\{a_{1}, \ldots, a_{k}\right\}}(T) M_{\left(a_{1}+1, \ldots, n-a_{k}\right)} \\
= & \sum_{\left\{a_{1}, \ldots, a_{k}\right\} \in 2^{[0, n-2]}} f_{\left\{a_{1}, \ldots, a_{k}\right\}}(T) \mathscr{O}_{0} M_{\left(a_{1}+1, \ldots, n-1-a_{k}\right)}=\mathscr{O}_{0} \mathrm{~F}(T), \\
\mathrm{F}\left(\mathscr{O}_{1} T\right)= & \sum_{\left\{a_{1}, \ldots, a_{k}\right\} \in 2^{[0, n-1]}} f_{\left\{a_{1}, \ldots, a_{k}\right\}}\left(\mathscr{O}_{1} T\right) M_{\left(a_{1}+1, a_{2}-a_{1}, \ldots, n-a_{k}\right)} \\
= & \sum_{\left\{a_{1}, \ldots, a_{k-1}, n-1\right\} \in 2^{[0, n-1]}} f_{\left\{a_{1}, \ldots, a_{k-1}\right\}}(T) M_{\left(a_{1}+1, \ldots, n-1-a_{k-1}, 1\right)} \\
= & \sum_{\left\{a_{1}, \ldots, a_{k-1}\right\} \in 2^{[0, n-2]}} f_{\left\{a_{1}, \ldots, a_{k-1}\right\}}(T) \mathscr{O}_{1} M_{\left(a_{1}+1, \ldots, n-1-a_{k-1}\right)}=\mathscr{O}_{1} \mathrm{~F}(T) .
\end{aligned}
$$

СлеДСтвиЕ 2.16. 1) Отображение Эренборга $\mathrm{F}: \mathcal{R} \rightarrow \mathcal{Q}$ sут является эпиморфизмом.

2) Флаговые числа $f_{S}, S \subseteq[0, n-2]$, как функции на группе $\mathcal{R}^{2 n}$ линейно независимь над $\mathbb{Q}$.

Этот результат впервые был получен в работе [31].

2.3. Алгебры Лейбница-Хопфа. Пусть $R$ - коммутативное ассоциативное кольцо с единицей.

ОПРЕДЕЛЕНИЕ 2.17. Алгеброй Лейбница-Хопфа называется ассоциативная алгебра Хопфа $\mathcal{H}$ с фиксированной конечной или счетной последовательностью мультипликативных образующих $H_{i}, i=1,2, \ldots$, и коумножением

$$
\Delta H_{n}=\sum_{i+j=n} H_{i} \otimes H_{j}, \quad H_{0}=1 .
$$


Универсальной алгеброй Лейбница-Хопфа называется алгебра Лейбница-Хопфа $\mathcal{A}$ с последовательностью мультипликативных образующих $A_{i}, i=1,2, \ldots$, такая, что для любой алгебры Лейбница-Хопфа $\mathcal{H}$ соответствие $A_{i} \rightarrow H_{i}$ определяет гомоморфизм алгебр Хопфа $\mathcal{A} \rightarrow \mathcal{H}$.

Рассмотрим свободную ассоциативную алгебру $\mathcal{Z}=\mathbb{Z}\left\langle Z_{1}, Z_{2}, \ldots\right\rangle$ с фиксированной последовательностью образующих $Z_{i}, i \in \mathbb{N}$.

УтВеРжДЕНИЕ 2.18. Алгебра $\mathcal{Z}$ имеет структуру алгебры Лейбнииа-Хоп$\oint a(\mathcal{Z}, \mu, \imath, \Delta, \varepsilon, \chi)$, где

- умножение $\mu: \mathcal{Z} \otimes \mathcal{Z} \rightarrow \mathcal{Z}$ определяется умножением в свободной ассоциативной алгебре;

- единица $\imath: \mathbb{Z} \rightarrow \mathcal{Z}$ переводит единицу $1 \in \mathbb{Z}$ в единицу $Z_{0}=1 \in \mathcal{Z}$;

- коумножение $\Delta: \mathcal{Z} \rightarrow \mathcal{Z} \otimes \mathcal{Z}$ задается формулой

$$
\Delta Z_{n}=\sum_{i+j=n} Z_{i} \otimes Z_{j}
$$

- коединица задается формулой

$$
\varepsilon\left(Z_{n}\right)= \begin{cases}1, & \text { если } n=0, \\ 0 \quad \text { в противном случае }\end{cases}
$$

- антипод $\chi: \mathcal{Z} \rightarrow \mathcal{Z}$ действует по формуле

$$
\chi\left(Z_{n}\right)=\sum_{\omega:|\omega|=n}(-1)^{l(\omega)} Z_{\omega}, \quad n \geqslant 1,
$$

где $Z_{\omega}=Z_{j_{1}} \cdots Z_{j_{k}}$ для композиции $\omega=\left(j_{1}, \ldots, j_{k}\right)$. ЗАмечаниЕ 2.19. Положим $\Phi(t)=1+Z_{1} t+Z_{2} t^{2}+\cdots=\sum_{k=0}^{n} Z_{k} t^{k}$. Тогда
формула коумножения равносильна условию

Антипод $\chi: \mathcal{Z} \rightarrow \mathcal{Z}$ должен удовлетворять уравнению

$$
1 \star \chi=\mu(1 \otimes \chi) \Delta=\imath \varepsilon=\mu(\chi \otimes 1) \Delta=\chi \star 1 .
$$

Следовательно,

$$
\Phi(t) \cdot \chi(\Phi(t))=1=\chi(\Phi(t)) \cdot \Phi(t)
$$

и элементы $\left\{\chi\left(Z_{n}\right)\right\}$ удовлетворяют рекуррентным формулам

$$
\chi\left(Z_{1}\right)=-Z_{1} ; \quad \chi\left(Z_{n+1}\right)+\chi\left(Z_{n}\right) Z_{1}+\cdots+\chi\left(Z_{1}\right) Z_{n}+Z_{n+1}=0, \quad n \geqslant 1 .
$$

Пользуясь формулой (36), мы получаем:

$$
\begin{gathered}
\chi(\Phi(t))=\frac{1}{\Phi(t)}=\sum_{k=0}^{\infty}(-1)^{k}(\Phi(t)-1)^{k} ; \\
\chi\left(Z_{n}\right)=\sum_{k=1}^{n}(-1)^{k} \sum_{j_{1}+\cdots+j_{k}=n, j_{i}>0} Z_{j_{1}} \cdots Z_{j_{k}}, \quad n \geqslant 1 .
\end{gathered}
$$

УтВеРЖДЕНИЕ 2.20. Алгебра Хопфа $\mathcal{Z}$ является универсалъной алгеброй Лейбница-Xопфа. 
Положим $\operatorname{deg} Z_{i}=2 i$. Пусть $\mathcal{M}$ - градуированно двойственная к $\mathcal{Z}$ алгебра Хопфа. Имеет место следующий известный факт.

УтвеРЖДЕНИЕ 2.21. Алгебра Хопфа квазисимметрических функций $\mathcal{Q} s y m$ изоморфна алгебре Хопфа $\mathcal{M}$.

Действительно, для композиции $\omega=\left(j_{1}, \ldots, j_{k}\right)$ определим элемент $m_{\omega} \in \mathcal{M}$ формулой

$$
\left\langle m_{\omega}, Z_{\sigma}\right\rangle=\delta_{\omega, \sigma},
$$

где $Z_{\sigma}=Z_{a_{1}} \cdots Z_{a_{l}}$ для композиции $\sigma=\left(a_{1}, \ldots, a_{l}\right)$. Тогда элементы $m_{\omega}$ умножаются так же, как квазисимметрические мономы $M_{\omega}$.

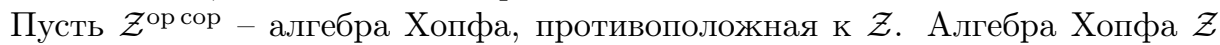

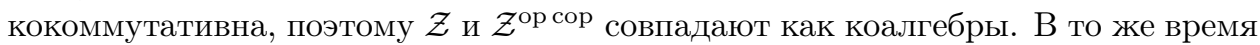

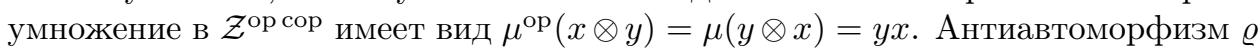
алгебры Хопфа $\mathcal{Z}$

$$
\varrho\left(Z_{a_{1}} \cdots Z_{a_{k}}\right)=Z_{a_{k}} \cdots Z_{a_{1}}
$$

обладает свойством $\Delta \varrho=(\varrho \otimes \varrho) \Delta$ и поэтому задает изоморфизм алгебр Хопфа $\mathcal{Z}$ и $\mathcal{Z}^{\text {ор сор }}$.

УТВеРЖДЕНИЕ 2.22. На колъце $\mathcal{Q}$ sym $=\mathcal{M}$ имеем $\varrho^{*}=*$.

Доказательство. Так как

$$
\left\langle\varrho^{*} M_{\omega}, Z_{\sigma}\right\rangle=\left\langle M_{\omega}, \varrho Z_{\sigma}\right\rangle=\left\langle M_{\omega}, Z_{\sigma^{*}}\right\rangle=\delta_{\omega, \sigma^{*}}=\delta_{\omega^{*}, \sigma}=\left\langle M_{\omega^{*}}, Z_{\sigma}\right\rangle,
$$

то $\varrho^{*} M_{\omega}=M_{\omega^{*}}=M_{\omega}^{*}$.

ОПРЕДЕЛЕНИЕ 2.23 [32]. (Левым) модулем Милнора $M$ (или модулем Xоn$\oint a)$ над алгеброй Хопфа $X$ называется алгебра с единицей $1 \in R$, которая также является (левым) модулем над $X$ таким, что $x(1)=\varepsilon(x) \cdot 1$ и

$$
x(u v)=\sum x_{n}^{\prime}(u) x_{n}^{\prime \prime}(v), \quad x \in X, \quad u, v \in M, \quad \Delta x=\sum x_{n}^{\prime} \otimes x_{n}^{\prime \prime} .
$$

Пусть $A$ - одно из колец $\mathcal{P}, \mathcal{R} \mathcal{P}$ или $\mathcal{R}$.

УтВеРЖДЕНИЕ 2.24. 1) Гомоморфизм $\mathscr{L}_{A}: \mathcal{Z} \rightarrow \mathcal{D}(A), Z_{k} \rightarrow d_{k}$, определяет на кольце $A$ структуру левого $\mathcal{Z}$-модуля Милнора.

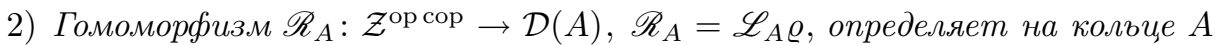
структуру правого $\mathcal{Z}$-модуля Милнора.

3) Отображение $\mathscr{L}_{\mathcal{R}}: \mathcal{Z} \rightarrow \mathcal{D}(\mathcal{R})$ является изоморфизмом колеи.

4) Коумножение $d_{k} \rightarrow \sum d_{i} \otimes d_{j}$ определяет на кольще $\mathcal{D}(\mathcal{R})$ структуру алгебры Лейбница-Хопфа. Кольщо $\mathcal{R}$ имеет каноническую структуру левого модуля Милнора над $\mathcal{D}(\mathcal{R})$.

ДокАзАтЕльство. Доказательство утверждений 1) и 2) следует из формулы $d_{k}\left(T^{\prime} T^{\prime \prime}\right)=\sum_{i+j=k}\left(d_{i} T^{\prime}\right)\left(d_{j} T^{\prime \prime}\right)$ (см. конец п. 1.3) и утверждения 1.63.

Чтобы доказать утверждение 3), покажем, что операторы $\left\{d_{k} \in \mathcal{D}(\mathcal{R})\right.$, $k \geqslant 1\}$ алгебраически независимы. Пусть $u\left(d_{1}, \ldots, d_{n}\right)=0 \in \mathcal{D}_{-2 n}(\mathcal{R})$, где $u=\sum u_{\omega} D_{\omega}$ - однородный некоммутативный полином. Тогда линейная функция $\mathcal{R}^{2 n} \rightarrow \mathbb{Z}$, переводящая $T$ в $\varepsilon\left(u\left(d_{1}, \ldots, d_{n}\right) T\right)$ и являющаяся линейной 
комбинацией различных флаговых чисел $f_{S}, S \subseteq[0, n-2]$, с коэффициентами $u_{\omega}$, тождественно равна нулю. Поэтому согласно следствию 2.16 все числа $u_{\omega}$ равны нулю.

Утверждение 4) непосредственно следует из утверждений 1) и 3).

Отметим, что моном $Z_{\omega}=Z_{j_{1}} \cdots Z_{j_{k}}$ при гомоморфизме $\mathscr{L}_{A}$ переходит в опеpaтор $D_{\omega}=d_{j_{1}} \cdots d_{j_{k}}$, а при гомоморфизме $\mathscr{R}_{A}-$ в оператор $D_{\omega^{*}}=d_{j_{k}} \cdots d_{j_{1}}$.

ОПРЕДЕЛЕНИЕ 2.25. Положим $\mathcal{U}=\mathcal{Z} / J_{\mathcal{U}}$, где двухсторонний хопфовский идеал $J_{\mathcal{U}}$ порожден элементами

$$
Z_{n}-Z_{1} Z_{n-1}+\cdots+(-1)^{n-1} Z_{n-1} Z_{1}+(-1)^{n} Z_{n}, \quad n \geqslant 2 .
$$

Все эти элементы являются коэффициентами ряда $\Phi(-t) \Phi(t)$, так что для алгебры Хопфа $\mathcal{U}$ имеет место формула $\Phi(-t) \Phi(t)=1$. Из соотношения (38) следует, что $\mathcal{U} \otimes \mathbb{Q} \simeq \mathbb{Q}\left\langle Z_{1}, Z_{3}, Z_{5}, \ldots\right\rangle$, и поэтому отображение $\mathcal{U} \stackrel{\operatorname{id} \otimes 1}{\longrightarrow} \mathcal{U} \otimes \mathbb{Q}$ является вложением (см. раздел 5$)$.

Обозначим через $U_{i}$ образ образующей $H_{i}$ при факторотображении $\mathcal{Z} \rightarrow \mathcal{U}$.

УтвеРЖДЕНИЕ 2.26. В алгебре Хопфа $\mathcal{U}$ имеет место формула

$$
\chi\left(U_{i}\right)=(-1)^{i} U_{i} .
$$

Алгебра Хопфа $\mathcal{U}$ является универсальной в категории алгебр Лейбница-Хопфа, удовлетворяющих условию (39).

ДокАЗАтЕльство. Сравнивая рекуррентную формулу для антипода (37) с формулой $(38)$ и пользуясь тем, что $\chi\left(U_{1}\right)=-U_{1}$, мы получаем, что $\chi\left(U_{i}\right)=$ $(-1)^{i} U_{i}$. Универсальность алгебры $\mathcal{U}$ следует из утверждения 2.20.

СлеДСТВИе 2.27. Отображения $\mathscr{L}_{\mathrm{R}}$ и $\mathscr{R}_{\mathrm{R}}$ определяют на колъце $\mathrm{R}$ структуры левого и правого модуля Милнора над алгеброй Хопфа $\mathcal{U}$.

Это следует из утверждений 2.24 и 1.67.

ЗАмечаниЕ 2.28. Так как $\varrho\left(J_{\mathcal{U}}\right)=J_{\mathcal{U}}$, то отображение $\varrho$ индуцирует корректно определенный антигомоморфизм $\varrho: \mathcal{U} \rightarrow \mathcal{U}$, который задает изоморфизм $\mathcal{U} \simeq \mathcal{U}^{\text {ор сор }}$.

Факторотображение $\mathcal{Z} \rightarrow \mathcal{Z} / J_{\mathcal{U}}$ является эпиморфизмом, поэтому двойственное ему отображение градуированных двойственных алгебр Хопфа $\mathcal{U}^{*} \rightarrow$ $\mathcal{Z}^{*}=\mathcal{Q} s y m$ является вложением. При этом образ алгебры Хопфа $\mathcal{U}^{*}$ в алгебре Хопфа $\mathcal{Q} s y m$ инвариантен относительно инволюции $\varrho^{*}=*$.

ОПРедЕЛЕНИЕ 2.29. Обозначим через $\mathcal{C}$ свободную коммутативную алгебру Лейбница-Хопфа от образующих $C_{i}$ степени $2 i$. Имеем $\mathcal{C}=\mathcal{Z} / J_{\mathcal{C}}$, где идеал $J_{\mathcal{C}}$ порожден элементами $Z_{i} Z_{j}-Z_{j} Z_{i}$.

УтвеРЖДЕНИЕ 2.30. Алгебра Хопфа $\mathcal{C}=\mathbb{Z}\left[C_{1}, C_{2}, \ldots\right]$ является универсальной в категории коммутативных алгебр Лейбница-Хопфа.

Алгебра Хопфа $\mathcal{C}$ является самодвойственной, причем градуированно двойственная к ней алгебра Хопфа $\mathcal{C}^{*}$ канонически изоморфна алгебре Хопфа симметрических функций $\mathbb{Z}\left[\sigma_{1}, \sigma_{2}, \ldots\right]=\operatorname{Sym}\left[t_{1}, t_{2}, \ldots\right] \subset \operatorname{Qsym}\left[t_{1}, t_{2}, \ldots\right]$, порожденной элементарными симметрическими функциями

$$
\sigma_{i}=M_{(1, \ldots, 1)}=\sum_{l_{1}<\cdots<l_{i}} t_{l_{1}} \cdots t_{l_{i}}
$$


Отображения $\mathcal{Z} \rightarrow \mathcal{C}$ и $\operatorname{Sym}\left[t_{1}, t_{2}, \ldots\right] \hookrightarrow \mathrm{Qsym}\left[t_{1}, t_{2}, \ldots\right]$ двойственны друг к другу, а изоморфизм $\mathcal{C} \simeq \mathcal{C}^{*}$ задается соответствием $C_{i} \rightarrow \sigma_{i}$.

\section{4. Алгебры Ли-Хопфа.}

ОПРЕДЕЛЕНИЕ 2.31. Алгеброй Ли-Хопфа называется алгебра Хопфа $\mathfrak{L}$ с фиксированной конечной или счетной последовательностью мультипликативных образующих $L_{i}, i=1,2, \ldots$, с коумножением

$$
\Delta L_{n}=1 \otimes L_{n}+L_{n} \otimes 1, \quad L_{0}=1 .
$$

Универсальной алгеброй Ли-Хопфа называется алгебра Ли-Хопфа $\mathcal{A}$ с набором мультипликативных образующих $A_{i}, i=1,2, \ldots$, такая, что для любой алгебры Ли-Хопфа $\mathfrak{L}$ соответствие $A_{i} \rightarrow L_{i}$ определяет гомоморфизм алгебр Хопфа $\mathcal{A} \rightarrow \mathfrak{L}$.

Рассмотрим свободную ассоциативную алгебру $\mathcal{W}=\mathbb{Z}\left\langle W_{1}, W_{2}, \ldots\right\rangle$ над кольцом целых чисел с фиксированной последовательностью образующих $W_{i}, i \in \mathbb{N}$.

УтВеРЖДЕНИЕ 2.32. Свободная ассоциативная алгебра $\mathcal{W}$ имеет структуру алгебры Ли-Хопфа $(\mathcal{W}, \mu, \imath, \Delta, \varepsilon, \chi)$, где

- умножение $\mu: \mathcal{W} \otimes \mathcal{W} \rightarrow \mathcal{W}$ определяется умножением в свободной ассоциативной алгебре;

- единица $: \mathbb{Z} \rightarrow \mathcal{W}$ переводит единичу $1 \in \mathbb{Z}$ в единичу $W_{0}=1 \in \mathcal{W}$

- коумножение $\Delta: \mathcal{W} \rightarrow \mathcal{W} \otimes \mathcal{W}$ задается формулой

$$
\Delta W_{n}=1 \otimes W_{n}+W_{n} \otimes 1 ;
$$

- коединица задается формулой $\varepsilon\left(W_{n}\right)= \begin{cases}1, & \text { если } n=0, \\ 0 & \text { в противном случае; }\end{cases}$

- антипод $\chi: \mathcal{W} \rightarrow \mathcal{W}$ действует по бормуле $\chi\left(W_{n}\right)=-W_{n}$.

УтвеРЖДЕНИЕ 2.33. Алгебра Хопфа $\mathcal{W}$ является универсалъной алгеброй Лu-Xonøa.

Положим $\operatorname{deg} W_{i}=2 i$ и обозначим через $\mathcal{N}$ градуированно двойственную к $\mathcal{W}$ алгебру Хопфа. Получаем так называемую тасовочную алгебру.

Соответствие

$$
1+Z_{1} t+Z_{2} t^{2}+\cdots=\exp \left(W_{1} t+W_{2} t^{2}+\cdots\right)
$$

определяет изоморфизм алгебр Хопфа $\mathcal{Z} \otimes \mathbb{Q} \simeq \mathcal{W} \otimes \mathbb{Q}$.

\section{3. Слова Линдона}

Известная теорема из теории алгебр Ли (см., например, [33]) утверждает, что алгебра $\mathcal{N} \otimes \mathbb{Q}$ является алгеброй полиномов от так называемых слов Линдона.

ОПРЕДЕЛЕНИЕ 3.1. Пусть $\left[a_{1}, \ldots, a_{n}\right] \in \mathbb{N}^{\infty}=\lim _{\longrightarrow} \mathbb{N}^{n}$, т. е. слово, состоящее из символов $a_{1}, \ldots, a_{n}, a_{i} \in \mathbb{N}$, где $\mathbb{N}$ - множество натуральных чисел. Считая пустое множество минимальным элементом, упорядочим такие слова лексикографически, т. е. $\left[a_{1}, \ldots, a_{n}\right]>\left[b_{1}, \ldots, b_{m}\right]$ тогда и только тогда, когда найдется такое $i$, что $a_{1}=b_{1}, \ldots, a_{i-1}=b_{i-1}, a_{i}>b_{i}, 1 \leqslant i \leqslant \min \{m, n\}$, либо $n>m$ и $a_{1}=b_{1}, \ldots, a_{m}=b_{m}$. 
Собственным концом слова $\left[a_{1}, \ldots, a_{n}\right]$ называется любое слово вида $\left[a_{i}, \ldots, a_{n}\right], \quad 1<i \leqslant n$. (Пустое слово и слово, состоящее из одного символа, не имеют собственных концов.)

Словом Линдона называется такое слово, которое меньше любого своего собственного конца. Например, слова $[1,1,2],[1,2,1,2,2],[1,3,1,5]$ является словами Линдона, а слова $[1,1,1,1],[1,2,1,2],[2,1]$ не являются. Положим $\mathrm{LYN}=\bigcup_{n \geqslant 0} \mathrm{LYN}^{2 n}$, где $\mathrm{LYN}^{2 n}-$ множество всех слов Линдона градуировки $2 n$.

Ясно, что понятие слов Линдона можно ввести для любого вполне упорядоченного конечного или бесконечного множества. Далее важную роль будут играть слова Линдона для множества $\{1,2\}$ и для множества odd всех нечетных натуральных чисел.

Следующий результат играет важную роль в приложениях слов Линдона.

Теорема 3.2 (факторизация Чена-Фокса-Линдона, см. [22], [34]). Каждое слово $w \in \mathbb{N}^{\infty}$ единственным образом разлагается в произведение невозрастающих слов Линдона, т.е. $w=u_{1} * \cdots * u_{k}, u_{i} \in \mathrm{LYN}, u_{1} \geqslant \cdots \geqslant u_{k}$.

Например, $[1,1,1,1]=[1] *[1] *[1] *[1],[1,2,1,2]=[1,2] *[1,2],[2,1]=[2] *[1]$.

Алгебра $\mathcal{N}$ аддитивно порождена словами из множества $\mathbb{N}^{\infty}$. При этом слово $w=\left[a_{1}, \ldots, a_{n}\right]$ соответствует линейному отображению $\mathcal{W} \rightarrow \mathbb{Z}$ такому, что $\left\langle\left[a_{1}, \ldots, a_{n}\right], W_{\omega}\right\rangle=\delta_{w, \omega}$, где $W_{\omega}=W_{b_{1}} \ldots W_{b_{l}}, \omega=\left(b_{1}, \ldots, b_{l}\right)$ и для слова $w=\left[a_{1}, \ldots, a_{n}\right]$

$$
\delta_{w, \omega}= \begin{cases}1, & \omega=\left(a_{1}, \ldots, a_{n}\right) ; \\ 0 & \text { в противном случае. }\end{cases}
$$

Умножение в алгебре $\mathcal{N}$ называется тасовочным умножением и имеет вид

$$
\left[a_{1}, \ldots, a_{n}\right] \times_{\mathrm{sh}}\left[a_{n+1}, \ldots, a_{m+n}\right]=\sum_{\sigma}\left[a_{\sigma(1)}, \ldots, a_{\sigma(n)}, a_{\sigma(n+1)}, \ldots, a_{\sigma(n+m)}\right],
$$

где $\sigma$ - перестановка из группы $S_{n+m}$ такая, что

$$
\sigma^{-1}(1)<\cdots<\sigma^{-1}(n) \quad \text { и } \quad \sigma^{-1}(n+1)<\cdots<\sigma^{-1}(n+m) .
$$

Например,

$$
\begin{aligned}
& {[1] \times \times_{\mathrm{sh}}[1] }=[1,1]+[1,1]=2[1,1] \\
& {[1] \times \times_{\mathrm{sh}}[2,3]=} {[1,2,3]+[2,1,3]+[2,3,1] } \\
& {[1,2] \times \times_{\mathrm{sh}}[1,2]=[1,2,1,2]+[1,1,2,2]+[1,1,2,2]+[1,1,2,2] } \\
&+[1,1,2,2]+[1,2,1,2]=2[1,2,1,2]+4[1,1,2,2] .
\end{aligned}
$$

Имеет место известный результат о структуре тасовочной алгебры.

Теорема 3.3. $\mathcal{N} \otimes \mathbb{Q}=\mathbb{Q}[\mathrm{LYN}]$, m. е. тасовочная алгебра над полем рациональных чисел является алгеброй полиномов от слов Линдона.

Доказательство вытекает из следующей теоремы, описывающей связь тасовочного умножения и факторизации Чена-Фокса-Линдона.

Теорема 3.4. Пусть $w-$ слово из $\mathbb{N}^{\infty} u w=u_{1} * \cdots * u_{m}$ - его разложение Чена-Фокса-Линдона. Тогда все слова, которые входят в разложение тасовочного произведения $u_{1} \times_{\mathrm{sh}} \cdots \times_{\mathrm{sh}} u_{m}$ с ненулевыми коэффициентами, не 
превосходят слова $w$ относительно лексикографического порядка, при этом слово $w$ входит в это произведение с ненулевым целочисленным коэфбициентом.

Используя этот результат, нетрудно доказать теорему о структуре тасовочной алгебры в случае произвольного подмножества $M=\left\{m_{1}, m_{2}, \ldots\right\} \subseteq \mathbb{N}$.

Пусть $\mathcal{W}_{M}=\mathbb{Z}\left\langle W_{m_{1}}, W_{m_{2}}, \ldots\right\rangle$ - свободная ассоциативная алгебра Ли-Хопфа и $\mathcal{N}_{M}$ - градуированная двойственная ей алгебра Хопфа. Обозначим через $\mathrm{LYN}_{M}$ соответствующее множество слов Линдона.

\section{TEOPEMA 3.5. $\mathcal{N}_{M} \otimes \mathbb{Q}=\mathbb{Q}\left[\mathrm{LYN}_{M}\right]$.}

ДокАЗАТЕЛьСтво. Покажем по индукции, что элементы множества $\mathrm{LYN}_{M}$ порождают всю алгебру $\mathcal{N}_{M} \otimes \mathbb{Q}$. Пусть $m_{1} \in M$ - минимальный элемент, тогда слово $\left[m_{1}\right]$ линдоново. Предположим, что все слова, лексикографически меньшие слова $w$, могут быть представлены как полиномы от элементов множества $\mathrm{LYN}_{M}$. Применяя предыдущую теорему к разложению Чена-Фокса-Линдона $w=u_{1} * \cdots * u_{m}$, мы получаем представление $u_{1} \times_{\mathrm{sh}} \cdots \times_{\mathrm{sh}} u_{m}=a w+($ остаток $)$, где $a \in \mathbb{N}$ и все слова из остатка лексикографически меньше слова $w$. По предположению индукции, остаток является полиномом от элементов множества $\operatorname{LYN}_{M}$. Поэтому $w \in \mathbb{Q}\left[\mathrm{LYN}_{M}\right]$.

Таким образом, элементы множества $\mathrm{LYN}_{M}$ порождают алгебру $\mathcal{N}_{M} \otimes \mathbb{Q}$. Так как каждое слово градуировки $2 n$ в алгебре $\mathcal{N}_{M}$ имеет единственное разложение Чена-Фокса-Линдона, то число мономов от слов Линдона $\mathrm{LYN}_{M}$ степени $2 n$ равно размерности градуированной компоненты алгебры $\mathcal{N}_{M}$ степени $2 n$. Следовательно, мономы от слов Линдона линейно независимы, слова Линдона алгебраически независимы и $\mathcal{N}_{M} \otimes \mathbb{Q} \simeq \mathbb{Q}\left[\mathrm{LYN}_{M}\right]$.

СлеДСтвиЕ 3.6. Алгебры Хопфа $\mathcal{N}_{\{12\}} \otimes \mathbb{Q} u \mathcal{N}_{\text {odd }} \otimes \mathbb{Q}$, градуированно двойственные к алгебрам Ли-Хопфа $\mathcal{W}_{\{12\}} \otimes \mathbb{Q}=\mathbb{Q}\left\langle W_{1}, W_{2}\right\rangle$ и $\mathcal{W}_{\text {odd }} \otimes \mathbb{Q}$, являются алгебрами полиномов от слов Линдона $\mathrm{LYN}_{\{12\}} u \mathrm{LYN}_{\mathrm{odd}}$ соответственно.

ТЕОРема 3.7. 1) Имеет место изоморфизм градуированных колеи, полиномов: $\mathcal{N}_{\{12\}} \otimes \mathbb{Q} \simeq \mathcal{N}_{\text {odd }} \otimes \mathbb{Q}[\beta]$, где $\operatorname{deg} \beta=4$.

2) $\operatorname{dim} \mathcal{N}_{\{12\}}^{2 n} \otimes \mathbb{Q}=c_{n}, n \geqslant 0$, следовательно, имеет место разложение производщего ряда для чисел Фибоначчи в бесконечное произведение:

$$
\frac{1}{1-t-t^{2}}=\sum_{n=0}^{\infty} c_{n} t^{n}=\prod_{i=1}^{\infty} \frac{1}{\left(1-t^{i}\right)^{k_{i}}},
$$

где $k_{n}=\left|\mathrm{LYN}_{\{12\}}^{2 n}\right|$ - число слов Линдона градуировки $2 n$ в множестве $\mathrm{LYN}_{\{12\}}$. При $n \neq 2$ оно равно числу слов Линдона градуировки $2 n$ в множестве $\mathrm{LYN}_{\text {odd }}$.

3) $k_{n+1} \geqslant k_{n} \geqslant N_{n}-2$, где $N_{n}$ - число представлений числа $n$ в виде суммы нечетных натуральных чисел.

ДокАЗАтельство. 1) Между множествами $\mathrm{LYN}_{\{12\}}^{2 n}$ и $\mathrm{LYN}_{\text {odd }}^{2 n}$ существует взаимно однозначное соответствие, при котором слово $[1,(2, \ldots, 2), 1,(2, \ldots, 2)$, $\ldots, 1,(2, \ldots, 2)]$, содержащее $k$ групп $(2, \ldots, 2)$, переходит в слово $\left[2 a_{1}+1, \ldots\right.$, $\left.2 a_{k}+1\right]$, где $a_{i}$ - число двоек в $i$-й группе. Это соответствие вместе с соответствием $[2] \rightarrow \beta$ задает требуемый изоморфизм алгебр полиномов. 
2) Размерность $\mu_{n}$ пространства $\operatorname{dim} \mathcal{N}_{\{12\}}^{2 n} \otimes \mathbb{Q}$ равна количеству различных слов из 1 и 2 градуировки $n$. Имеем $\mu_{0}=\mu_{1}=1$. В рассматриваемом случае слово $w$ имеет вид $\left[1, w^{\prime}\right]$ или $\left[2, w^{\prime \prime}\right]$, поэтому $\mu_{n+1}=\mu_{n}+\mu_{n-1}, n \geqslant 1$. Таким образом, $\mu_{n}=c_{n}$.

3) Имеем: $k_{1}=k_{2}=k_{3}=1$.

Пусть $n \geqslant 3$ и $w=\left[a_{1}, \ldots, a_{k}\right] \in \operatorname{LYN}_{\{12\}}^{2 n}$. Тогда $[1, w]=\left[1, a_{1}, \ldots, a_{k}\right] \in$ $\operatorname{LYN}_{\{12\}}^{2(n+1)}$. Действительно, для любого собственного конца $\left[a_{i}, \ldots, a_{k}\right], i \geqslant 1$, если $a_{i}>1$, то $\left[a_{i}, \ldots, a_{k}\right]>[1, w]$. Если $a_{i}=1$, то $i \neq k$ и $\left[a_{i+1}, \ldots, a_{k}\right]>$ $\left[a_{1}, \ldots, a_{k}\right]$, так как $w-$ слово Линдона. Таким образом, $k_{n+1} \geqslant k_{n}$.

Любое разложение $n=d_{1}+\cdots+d_{k}$ в сумму нечетных натуральных чисел $d_{1} \leqslant \cdots \leqslant d_{k}$, за исключением случая $k>1, d_{1}=\cdots=d_{k}=d$, дает слово Линдона $\left[d_{1}, \ldots, d_{k}\right] \in \mathrm{LYN}_{\text {odd }}$.

Разложению, состоящему из одинаковых слагаемых $d$, при $d \geqslant 5$ можно поставить в соответствие слово Линдона $[1, d-2,1, d, \ldots, d]$. При $d=1$ или $d=3$ мы получаем два разложения, которым может не соответствовать никакого слова Линдона, следовательно, $k_{n} \geqslant N_{n}-2$.

ПримеР 3.8. Пусть $n=6$. Тогда

$$
6=1+5=3+3=1+1+1+3=1+1+1+1+1+1 .
$$

Таким образом, $k_{6}=2$ и $N_{6}=4$. Следовательно, оценка из пункта 3$)$ неулучшаема.

ПримеР 3.9. Для малых $n$ мы имеем:

\begin{tabular}{|c|c|c|}
\hline$n$ & $\mathrm{LYN}_{\{12\}}^{2 n}$ & $\mathrm{LYN}_{\text {odd }}^{2 n}$ \\
\hline 3 & {$[1,2]$} & {$[3]$} \\
4 & {$[1,1,2]$} & {$[1,3]$} \\
5 & {$[1,2,2],[1,1,1,2]$} & {$[5],[1,1,3]$} \\
6 & {$[1,1,2,2],[1,1,1,1,2]$} & {$[1,5],[1,1,1,3]$} \\
7 & {$[1,2,2,2],[1,1,1,2,2],[1,1,2,1,2],[1,1,1,1,1,2]$} & {$[7],[1,1,5],[1,3,3],[1,1,1,1,3]$} \\
\hline
\end{tabular}

При этом

$$
\begin{aligned}
(1-t)\left(1-t^{2}\right) & =1-t-t^{2}+t^{3} ; \\
(1-t)\left(1-t^{2}\right)\left(1-t^{3}\right) & =1-t-t^{2}+t^{4}+t^{5}-t^{6} ; \\
(1-t)\left(1-t^{2}\right)\left(1-t^{3}\right)\left(1-t^{4}\right) & =1-t-t^{2}+2 t^{5}-t^{8}-t^{9}+t^{10} ; \\
(1-t)\left(1-t^{2}\right)\left(1-t^{3}\right)\left(1-t^{4}\right)\left(1-t^{5}\right)^{2} & =1-t-t^{2}+2 t^{6}+2 t^{7}-t^{8}-t^{9}-2 t^{10} \\
& -t^{11}-t^{12}+2 t^{13}+2 t^{14}-t^{18}-t^{19}+t^{20} .
\end{aligned}
$$

ЗАмЕчАНИЕ 3.10. (i) Из классических результатов следует, что произведение (41) абсолютно сходится при $|t|<(\sqrt{5}-1) / 2$.

(ii) Известно, что число разложений натурального числа $n$ в сумму нечетных натуральных чисел равно числу разложений числа $n$ в сумму различных натуральных чисел. 
ЗАмЕЧАНИЕ 3.11. Числа $k_{i}$ можно найти следующим образом:

$$
-\log \left(1-t-t^{2}\right)=-\sum_{i=1}^{\infty} k_{i} \log \left(1-t^{i}\right), \quad \sum_{n=1}^{\infty} \frac{1}{n} \sum_{j=0}^{n}\left(\begin{array}{c}
n \\
j
\end{array}\right) t^{n+j}=\sum_{i=1}^{\infty} k_{i} \sum_{r=1}^{\infty} \frac{t^{r i}}{r} .
$$

Тогда для любого $N$

$$
\sum_{j=0}^{[N / 2]} \frac{\left(\begin{array}{c}
N-j \\
j
\end{array}\right)}{N-j}=\frac{1}{N} \sum_{i \mid N} i k_{i}
$$

Используя формулу обращения Мёбиуса, мы получаем

$$
N k_{N}=\sum_{d \mid N}\left(d \sum_{j=0}^{[d / 2]} \frac{\left(\begin{array}{c}
d-j \\
j
\end{array}\right)}{d-j}\right) \mu\left(\frac{N}{d}\right)
$$

где $\mu(n)$ - функция Мёбиуса, т. е.

$$
\mu(n)= \begin{cases}1, & n=1 ; \\ (-1)^{r}, & n=p_{1} \cdots p_{r},\left\{p_{i}\right\}-\text { различные простые числа; } \\ 0, & n \text { содержит в разложении на простые множители квадраты. }\end{cases}
$$

Например, если $N=p$ - простое число, то

$$
p k_{p}=-1+p \sum_{j=0}^{[p / 2]} \frac{\left(\begin{array}{c}
p-j \\
j
\end{array}\right)}{p-j}, \quad k_{p}=\sum_{j=1}^{[p / 2]} \frac{\left(\begin{array}{c}
p-j \\
j
\end{array}\right)}{p-j} .
$$

Каждое слагаемое является целым числом, так как число

$$
\left(\begin{array}{c}
p-j \\
j
\end{array}\right)=\left(\begin{array}{c}
p-j-1 \\
j-1
\end{array}\right) \frac{p-j}{j}
$$

целое и числа $(p-j)$ и $j$ взаимно просты. Имеем: $k_{5}=2, k_{7}=4, k_{11}=18$ и т. д.

ЗАмечАнИЕ 3.12. Отметим, что, несмотря на изоморфизм алгебр Хопфа $\mathcal{N} \otimes \mathbb{Q} \simeq \mathcal{Q} s y m \otimes \mathbb{Q}$ и положительное решение гипотезы Диттерса, алгебра Хопфа $\mathcal{N}$ не является алгеброй полиномов над кольцом целых чисел.

\section{4. Топологическая реализация алгебр Хопфа}

Э. Бейкер и Б. Рихтер в [21] указали топологическую интерпретацию кольца квазисимметрических функций и в качестве следствия получили доказательство теоремы Хазевинкеля, используя фундаментальные результаты алгебраической топологии.

В этом разделе мы будем рассматривать для клеточных комплексов $X$ группы гомологий $\mathrm{H}_{*}(X)$ и когомологий $\mathrm{H}^{*}(X)$ с целыми коэффициентами. Предположим, что группы $\mathrm{H}_{*}(X)$ не имеют кручения.

Диагональное отображение $X \rightarrow X \times X$ задает на $\mathrm{H}_{*}(X)$ структуру градуированной коалгебры с коумножением $\Delta: \mathrm{H}_{*}(X) \rightarrow \mathrm{H}_{*}(X) \otimes \mathrm{H}_{*}(X)$ и двойственную структуру градуированной алгебры на $\mathrm{H}^{*}(X)$ с умножением $\Delta^{*}: \mathrm{H}^{*}(X) \otimes$ $\mathrm{H}^{*}(X) \rightarrow \mathrm{H}^{*}(X)$. 
В случае, когда $X$ является $H$-пространством с умножением $\mu: X \times X \rightarrow X$, мы получаем на коалгебре $\mathrm{H}_{*}(X)$ структуру градуированной алгебры Хопфа с умножением Понтрягина $\mu_{*}: \mathrm{H}_{*}(X) \otimes \mathrm{H}_{*}(X) \rightarrow \mathrm{H}_{*}(X)$. При этом кольцо когомологий $\mathrm{H}^{*}(X)$ получает структуру градуированно двойственной алгебры Хопфа с диагональным отображением $\mu^{*}: \mathrm{H}^{*}(X) \rightarrow \mathrm{H}^{*}(X) \otimes \mathrm{H}^{*}(X)$.

Для любого пространства $Y$ пространство петель $X=\Omega Y$ является $H$-пространством. Непрерывное отображение $f: Y_{1} \rightarrow Y_{2}$ индуцирует отображение $H$-пространств $\Omega f: X_{1} \rightarrow X_{2}$, где $X_{i}=\Omega Y_{i}$. Таким образом, для любого пространства $Y$ такого, что пространство петель $X=\Omega Y$ не имеет кручения в гомологиях, мы получаем алгебру Хопфа $\mathrm{H}_{*}(X)$. Это соответствие является функториальным, т. е. непрерывное отображение $f: Y_{1} \rightarrow Y_{2}$ индуцирует гомоморфизм алгебр Хопфа $f_{*}: \mathrm{H}_{*}\left(X_{1}\right) \rightarrow \mathrm{H}_{*}\left(X_{2}\right)$.

Пусть $\Sigma X$ - надстройка над пространством $X$. Согласно теореме БоттаСамельсона [35], кольцо $\mathrm{H}_{*}(\Omega \Sigma X)$ является свободной ассоциативной алгеброй $T\left(\widetilde{\mathrm{H}}_{*}(X)\right)$, порожденной абелевой группой $\widetilde{\mathrm{H}}_{*}(X)$. Эта конструкция функториальна, т. е. непрерывное отображение $f: X_{1} \rightarrow X_{2}$ индуцирует гомоморфизм соответствующих тензорных алгебр, индуцированный гомоморфизмом $f_{*}: \widetilde{\mathrm{H}}_{*}\left(X_{1}\right) \rightarrow \widetilde{\mathrm{H}}_{*}\left(X_{2}\right)$ (см. детали в [36]).

Мы будем обозначать элементы кольца $\mathrm{H}_{*}(\Omega \Sigma X)$ символами $\left(a_{1}|\cdots| a_{n}\right)$, где $a_{i} \in \widetilde{\mathrm{H}}_{*}(X)$. Так как диагональное отображение $\Sigma X \rightarrow \Sigma X \times \Sigma X$ дает отображение $H$-пространств $\Delta: \Omega \Sigma X \rightarrow \Omega \Sigma X \times \Omega \Sigma X$, то по теореме ЭйленбергаЗильбера мы получаем, что

$$
\Delta_{*}\left(a_{1}|\cdots| a_{n}\right)=\left(\Delta_{*} a_{1}|\cdots| \Delta_{*} a_{n}\right), \quad \text { где }\left(a_{1} \otimes b_{1} \mid a_{2} \otimes b_{2}\right)=\left(a_{1} \mid a_{2}\right) \otimes\left(b_{1} \mid b_{2}\right) .
$$

Для связного пространства $X$ имеется хорошая комбинаторная модель пространства $\Omega \Sigma X$, а именно конструкция Джеймса $J X$. После одной надстройки возникает расщепление:

$$
\Sigma \Omega \Sigma X \sim \Sigma J X \sim \bigvee_{n \geqslant 1} \Sigma X^{(n)},
$$

где $X^{(n)}$ обозначает $n$-кратную приведенную степень $X \wedge \cdots \wedge X$ пространства $X$.

ПримеР 4.1. Существует гомотопическая эквивалентность

$$
\Sigma \Omega \Sigma S^{2} \rightarrow \bigvee_{n \geqslant 1} \Sigma\left(S^{2}\right)^{(n)} \simeq \Sigma\left(\bigvee_{n \geqslant 1} S^{2 n}\right)
$$

Следовательно, существует отображение $H$-пространств

$$
\Omega \Sigma\left(\Omega \Sigma S^{2}\right) \rightarrow \Omega \Sigma\left(\bigvee_{n \geqslant 1} S^{2 n}\right),
$$

которое является гомотопической эквивалентностью.

Используя классические топологические результаты, опишем топологические реализации алгебр Хопфа, которые встречаются в нашем обзоре.

Пусть $\mathbb{C} P^{\infty}=\lim \mathbb{C} P^{n}$ и $B U=\lim B U(n)$, где $\mathbb{C} P^{n}-n$-мерное комплексное проективное пространство и $B U(n)$ - бесконечномерное комплексное многообразие Грассмана $n$-мерных подпространств. 
I. 1) $\mathrm{H}_{*}\left(\mathbb{C} P^{\infty}\right)$ - алгебра разделенных степеней $\mathbb{Z}\left[u_{1}, u_{2}, \ldots\right] / I$, где идеал $I$ порожден соотношениями $u_{i} u_{j}-\left(\begin{array}{c}i+j \\ i\end{array}\right) u_{i+j}$ и $\Delta u_{n}=\sum_{k=0}^{n} u_{k} \otimes u_{n-k}$.

2) $\mathrm{H}^{*}\left(\mathbb{C} P^{\infty}\right)=\mathbb{Z}[u]$ с коумножением $\Delta u=1 \otimes u+u \otimes 1$.

II. $\mathrm{H}_{*}\left(\Omega \Sigma \mathbb{C} P^{\infty}\right) \simeq T\left(\widetilde{\mathrm{H}}_{*}\left(\mathbb{C} P^{\infty}\right)\right)=\mathbb{Z}\left\langle u_{1}, u_{2}, \ldots\right\rangle$ с коумножением $\Delta u_{n}=$ $\sum u_{i} \otimes u_{j}$, индуцированным диагональным отображением пространства $\Omega \Sigma \mathbb{C} P^{\infty}$.

Теорема 4.2 [21]. Следующие алгебры Хопфа изоморфны:

$\mathrm{H}_{*}\left(\Omega \Sigma \mathbb{C} P^{\infty}\right) \simeq \mathcal{Z}=\mathbb{Z}\left\langle Z_{1}, Z_{2}, \ldots\right\rangle, \quad \mathrm{H}^{*}\left(\Omega \Sigma \mathbb{C} P^{\infty}\right) \simeq \mathcal{M}=\operatorname{Qsym}\left[t_{1}, t_{2}, \ldots\right]$.

III. $\mathrm{H}_{*}(B U) \simeq \mathrm{H}^{*}(B U) \simeq \mathbb{Z}\left[\sigma_{1}, \sigma_{2}, \ldots\right] \simeq \mathcal{C}$. Это самодвойственная алгебра Хопфа симметрических функций. В когомологиях функции $\sigma_{i}$ представлены классами Чженя.

IV. 1) $\mathrm{H}_{*}\left(\Omega \Sigma S^{2}\right)=\mathrm{H}_{*}\left(\Omega S^{3}\right)=\mathbb{Z}[w]-$ кольцо многочленов о переменной $w$, $\operatorname{deg} w=2$, с коумножением $\Delta w=1 \otimes w+w \otimes 1$.

2) $\mathrm{H}^{*}\left(\Omega \Sigma S^{2}\right)=\mathbb{Z}\left[u_{1}, u_{2}, \ldots\right] / I-$ алгебра разделенных степеней. Таким образом, алгебры Хопфа $\mathrm{H}^{*}\left(\Omega \Sigma S^{2}\right)$ и $\mathrm{H}_{*}\left(\mathbb{C} P^{\infty}\right)$ изоморфны.

V. $\mathrm{H}_{*}\left(\Omega \Sigma\left(\Omega \Sigma S^{2}\right)\right) \simeq \mathbb{Z}\left\langle w_{1}, w_{2}, \ldots\right\rangle-$ свободная ассоциативная алгебра с коумножением $\Delta w_{n}=\sum_{k=0}^{n}\left(\begin{array}{l}n \\ k\end{array}\right) w_{k} \otimes w_{n-k}$.

VI. $\mathrm{H}_{*}\left(\Omega \Sigma\left(\bigvee S^{2 n}\right)\right) \simeq \mathbb{Z}\left\langle\xi_{1}, \xi_{2}, \ldots\right\rangle-$ свободная ассоциативная алгебра с коумножением $\Delta \xi_{n}=1 \otimes \xi_{n}+\xi_{n} \otimes 1$. Таким образом, алгебра Хопфа $\mathrm{H}_{*}\left(\Omega \Sigma\left(\bigvee S^{2 n}\right)\right)$ дает топологическую реализацию универсальной алгебры Ли-Хопфа $\mathcal{W}$.

Гомотопическая эквивалентность $a: \Omega \Sigma\left(\bigvee S^{2 n}\right) \rightarrow \Omega \Sigma\left(\Omega \Sigma S^{2}\right)$ индуцирует изоморфизм градуированных алгебр Хопфа $a_{*}: \mathbb{Z}\left\langle\xi_{1}, \xi_{2}, \ldots\right\rangle \rightarrow$ $\mathbb{Z}\left\langle w_{1}, w_{2}, \ldots\right\rangle$.

Например, $a_{*} \xi_{1}=w_{1}, a_{*} \xi_{2}=w_{2}-\left(w_{1} \mid w_{1}\right), a_{*} \xi_{3}=w_{3}-3\left(w_{2} \mid w_{1}\right)+2\left(w_{1}\left|w_{1}\right| w_{1}\right)$.

Используя топологические результаты, мы получили изоморфизм над $\mathbb{Z}$ двух структур алгебры Хопфа на свободной ассоциативной алгебре с коумножениями

$$
\Delta w_{n}=\sum_{k=0}^{n}\left(\begin{array}{l}
n \\
k
\end{array}\right) w_{k} \otimes w_{n-k} \quad \text { и } \quad \Delta \xi_{n}=1 \otimes \xi_{n}+\xi_{n} \otimes 1 .
$$

Этот результат интересен и с топологической точки зрения, так как элементы $\left(w_{n}-a_{*} \xi_{n}\right), n \geqslant 2$, являются препятствиями к денадстройке гомотопической эквивалентности

$$
\Sigma\left(\Omega \Sigma S^{2}\right) \rightarrow \Sigma\left(\bigvee S^{2 n}\right)
$$

Мы имеем коммутативную диаграмму:

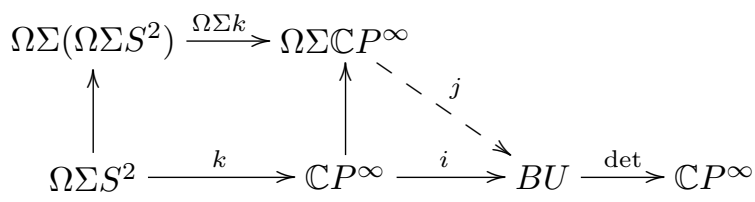


Здесь мы использовали следующие обозначения:

(a) $i$ - вложение $\mathbb{C} P^{\infty}=B U(1) \subset B U$;

(b) отображение $j$ возникает из свойства универсальности пространства $\Omega \Sigma \mathbb{C} P^{\infty}$ как свободного $H$-пространства;

(c) отображение $\mathbb{C} P^{\infty} \rightarrow \Omega \Sigma \mathbb{C} P^{\infty}$ индуцировано тождественным отображением $\Sigma \mathbb{C} P^{\infty} \rightarrow \Sigma \mathbb{C} P^{\infty}$;

(d) отображение $k: \Omega \Sigma S^{2} \rightarrow \mathbb{C} P^{\infty}$ отвечает образующей в $\mathrm{H}^{2}\left(\Omega \Sigma S^{2}\right)=\mathbb{Z}$;

(e) det - отображение классифицирующих пространств $B U \rightarrow B U(1)$, индуцированное отображениями $\operatorname{det}: U(n) \rightarrow U(1), n=1,2, \ldots$.

Как мы уже отмечали, алгебра Хопфа $\mathrm{H}^{*}(B U)$ изоморфна алгебре Хопфа симметрических функций (при этом классы Чженя соответствуют элементарным симметрическим функциям) и является самодвойственной:

$$
\mathrm{H}^{*}(B U) \simeq \mathrm{H}_{*}(B U) \simeq \operatorname{Sym}\left[t_{1}, t_{2}, \ldots\right]=\mathbb{Z}\left[\sigma_{1}, \sigma_{2}, \ldots\right] .
$$

Представителями мультипликативных образующих алгебры $\mathrm{H}^{*}(B U)$ являются образы при отображении $i$ фундаментальных циклов [C $\left.P^{n}\right]$. Таким образом, гомоморфизм $j_{*}$ является эпиморфизмом, а $j^{*}$ - мономорфизмом. Имеем:

- гомоморфизм $j_{*}$ соответствует факторизации алгебры некоммутативных полиномов $\mathcal{Z}$ по коммутаторам $Z_{i} Z_{j}-Z_{j} Z_{i}$;

- гомоморфизм $j^{*}$ соответствует включению $\operatorname{Sym}\left[t_{1}, t_{2}, \ldots\right]$ в кольцо квазисимметрических функций Qsym $\left[t_{1}, t_{2}, \ldots\right]$ и $j^{*}\left(\sigma_{i}\right)=M_{(1, \ldots, 1)}$;

- гомоморфизм $k^{*}$ соответствует включению $\mathbb{Z}[u]$ в алгебру разделенных степеней и $k^{*}(u)=u_{1}$;

- композиция $\operatorname{det}_{*} j_{*}$ соответствует гомоморфизму алгебры $\mathcal{Z}$ в алгебру $\mathbb{Z}\left[u_{1}, \ldots, u_{n}\right] / I$ и отвечает отображению $\left.Z_{k} \rightarrow d_{k}\right|_{\mathcal{P}_{\mathrm{s}}}$ алгебры $\mathcal{Z}$ в алгебpy $\mathcal{D}\left(\mathcal{P}_{\mathrm{s}}\right)$.

\section{5. Структура алгебры операторов граней $\mathcal{D}$}

Теорема 5.1. Гомоморфизм $\mathscr{L}_{\mathrm{R}}: \mathcal{Z} \rightarrow \mathcal{D}(\mathrm{R})$, переводящий $Z_{k}$ в $d_{k}$, индуиирует изоморфизм градуированных колеи,

$$
\mathcal{D}(\mathrm{R}) \simeq \mathcal{U}=\mathcal{Z} / J_{\mathcal{U}}
$$

где $\mathcal{U}$ - универсальная алгебра Хопфа в категории алгебр Лейбница-Хопфа с анmuподом $\chi\left(H_{i}\right)=(-1)^{i} H_{i}$.

ДокАзАТЕЛьство. Нам потребуется следующий результат.

Лемма 5.2. Пусть $D \in \mathcal{D}_{-2 k}(\mathrm{R})$. Тогда существуют однозначно определенные полиномы $u, u^{\prime}, w, w^{\prime}$ от некоммутирующих переменных такие, что операторь вида

$$
\begin{aligned}
& \widehat{D}=u\left(d_{2}, d_{3}, \ldots, d_{k}\right)+d w\left(d_{2}, d_{3}, \ldots, d_{k-1}\right), \\
& \widetilde{D}=u^{\prime}\left(d_{2}, d_{3}, \ldots, d_{k}\right)+w^{\prime}\left(d_{2}, d_{3}, \ldots, d_{k-1}\right) d
\end{aligned}
$$

действуют на каждом пространстве $\mathrm{R}^{2 n}, n \geqslant k$, так же, как оператор $D$. 
Доказательство. Для $k=0$ и $k=1$ утверждение верно. Утверждение верно и для $k=2$, так как $d^{2}=2 d_{2}$. Пусть $k \geqslant 3$. Из соотношений (19) вытекает формула

$$
d d_{i}-(-1)^{i} d_{i} d=\left(d_{2} d_{i-1}-d_{3} d_{i-2}+\cdots+(-1)^{i-1} d_{i-1} d_{2}\right)+\left(1+(-1)^{i+1}\right) d_{i+1} .
$$

Используя соотношение $d^{2}=2 d_{2}$ и то, что в правой части этой формулы оператор $d$ отсутствует, мы получаем существование требуемых операторов (43) и (44).

Допустим, что $u\left(d_{2}, \ldots, d_{k}\right)+d w\left(d_{2}, \ldots, d_{k}\right)=0$, где $u=\sum a_{\omega} D_{\omega},|\omega|=k$, и $w=\sum b_{\omega} D_{\omega},|\omega|=k-1$. Тогда для любого $n$-мерного многогранника $P^{n}$, $n \geqslant k$, имеем: $\xi_{1}(u+d w) P^{n}=0, \varepsilon_{1}(u+d w) P^{n}=0$. Так как оператор $u+d w$ имеет степень $-2 k$, то из этого следует, что

$$
\begin{aligned}
\sum_{|\omega|=k} a_{\omega} f_{\left\{n-k, n-k+j_{1}, n-k+j_{1}+j_{2}, \ldots, n-j_{l}\right\}}\left(P^{n}\right) & \\
& +\sum_{|\omega|=k-1} b_{\omega} f_{\left\{n-k, n-k+1, n-k+1+j_{1}, \ldots, n-j_{l}\right\}}\left(P^{n}\right)=0 .
\end{aligned}
$$

Пользуясь соотношениями Байер-Биллера, мы получаем

$$
\begin{aligned}
& f_{\left\{n-k, n-k+1, n-k+1+j_{1}, \ldots, n-j_{l}\right\}} \\
& =(-1)^{n-k-1}\left(\sum_{j=0}^{n-k-1}(-1)^{j} f_{\left\{j, n-k+1, n-k+1+j_{1}, \ldots, n-j_{l}\right\}}\right) \\
& \quad+\left(1+(-1)^{n-k}\right) f_{\left\{n-k+1, n-k+1+j_{1}, \ldots, n-j_{l}\right\}} .
\end{aligned}
$$

Теперь все встречающиеся множества $\left\{n-k, n-k+j_{1}, n-k+j_{1}+j_{2}, \ldots, n-j_{l}\right\}$, $\left\{j, n-k+1, n-k+j_{1}, \ldots, n-j_{l}\right\}$ и $\left\{n-k+1, n-k+1+j_{1}, \ldots, n-j_{l}\right\}$ для всех возможных композиций $\omega$ различны и принадлежат семейству $\Psi^{n}$.

В случае *-кольца $\mathcal{R} \mathcal{P}$ и многогранника $P^{k-1}$ размерности $n=k-1$ индекс $n-k=-1$ в выражении (45) исчезает и оно принимает вид

$$
\sum_{|\omega|=k} a_{\omega} f_{j_{1}-1, j_{1}+j_{2}-1, \ldots, n-j_{l}}\left(P^{n}\right)+\sum_{|\omega|=k-1} b_{\omega} f_{0, j_{1}, \ldots, n-j_{l}}\left(P^{n}\right)=0 .
$$

И в этом случае множества $\left\{j_{1}-1, j_{1}+j_{2}-1, \ldots, n-j_{l}\right\},\left\{0, j_{1}, \ldots, n-j_{l}\right\}$ для всевозможных композиций $\omega$ различны и принадлежат семейству $\Psi^{n}$.

Так как векторы $\left\{f_{S}(Q), S \in \Psi^{n}\right\}, Q \in \Omega^{n}$, линейно независимы, то все коэффициенты $a_{\omega}$ и $b_{\omega}$ равны нулю. Поэтому представление (43) единственно.

Мы получаем, что мономы

$$
D_{\omega}=d_{j_{1}} \ldots d_{j_{l}}, \quad|\omega|=k, \quad j_{i} \geqslant 2, \quad \text { и } \quad d D_{\omega}=d d_{j_{1}, \ldots, j_{l}}, \quad|\omega|=k-1, \quad j_{i} \geqslant 2,
$$

образуют базис абелевой группы $\mathcal{D}\left(\mathrm{R}^{2 n}, \mathrm{R}^{2(n-k)}\right), n \geqslant k$.

Каждый моном $d D_{\omega},|\omega|=k-1, j_{i} \geqslant 2$, может быть представлен в виде целочисленной комбинации мономов $D_{\omega^{\prime}}$ и $D_{\omega^{\prime}} d$, поэтому мономы вида $D_{\omega}, D_{\omega} d$ тоже образуют базис. Следовательно, представление (44) тоже единственно.

Теперь докажем теорему 5.1. Отображение $\mathscr{L}_{\mathrm{R}}: \mathcal{Z} \rightarrow \mathcal{D}(\mathrm{R})$ является градуированным эпиморфизмом. Пусть $z=\sum_{|\omega|=k} a_{\omega} Z_{\omega} \in \mathcal{Z}$ - элемент градуировки $2 k$
такой, что $\mathscr{L}_{\mathrm{R}} z=0$. 
Пользуясь соотношениями (38), мы можем (как в доказательстве леммы 5.2) представить элемент $z$ в виде

$$
z=\sum_{|\omega|=k, j_{i} \geqslant 2} a_{\omega}^{\prime} Z_{\omega}+\sum_{|\omega|=k-1, j_{i} \geqslant 2} b_{\omega} Z_{\omega} Z_{1}+z^{\prime}, \quad z^{\prime} \in J_{\mathcal{U}} .
$$

В силу утверждения 1.67 идеал $J_{\mathcal{U}}$ принадлежит ядру отображения $\mathscr{L}_{\mathrm{R}}$, поэтому $\mathscr{L}_{\mathrm{R}} z=\mathscr{L}_{\mathrm{R}} z^{\prime}=0=\mathscr{L}_{\mathrm{R}}\left(z-z^{\prime}\right)$. Следовательно,

$$
\sum_{|\omega|=k, j_{i} \geqslant 2} a_{\omega}^{\prime} D_{\omega}+\sum_{|\omega|=k-1, j_{i} \geqslant 2} b_{\omega} D_{\omega} d=0 .
$$

По лемме 5.2 все коэффициенты $a_{\omega}^{\prime}$ и $b_{\omega}^{\prime}$ равны нулю. Поэтому $z=z^{\prime} \in J_{\mathcal{U}}$.

Таким образом, мы доказали, что $\operatorname{Ker} \mathscr{L}_{\mathrm{R}}=J_{\mathcal{U}}$. Отсюда $\mathcal{Z} / J_{\mathcal{U}} \simeq \mathcal{D}(\mathrm{R})$.

ЗАмечАнИЕ 5.3. Мы видим, что соответствие $d_{k} \rightarrow d_{k}$ определяет изоморфизм $\mathcal{D}(\mathcal{R} \mathcal{P}) \rightarrow \mathcal{D}(\mathcal{P})$, поэтому далее мы будем обозначать эти алгебры единым символом $\mathcal{D}$, а гомоморфизмы $\mathscr{L}_{\mathrm{R}}$ и $\mathscr{R}_{\mathrm{R}}-$ символами $\mathscr{L}$ и $\mathscr{R}$.

СлЕДСТвиЕ 5.4. Кольцо $\mathcal{D}$ не имеет кручения.

СлЕДСТвиЕ 5.5. Коумножение $d_{k} \rightarrow \sum d_{i} \otimes d_{j}$ определяет на кольще $\mathcal{D}$ структуру алгебры Лейбница-Хопфа. Алгебра Хопфа $\mathcal{D}$ изоморфна алгебре Хопфа $\mathcal{U}$, универсальной в категории алгебр Лейбнища-Хопфа с антиподом $\chi\left(H_{i}\right)=(-1)^{i} H_{i}$. Кольцо $\mathrm{R}$ имеет каноническую структуру левого модуля Милнора над алгеброй Хопфа $\mathcal{D}$.

При помощи леммы 5.2 можно показать, что $\mathcal{D}\left(\mathrm{R}^{[2 n]}\right) \simeq \mathcal{Z} / J_{\mathcal{D}(\mathrm{R}[2 n])}$, где идеал $J_{\mathcal{D}\left(\mathrm{R}^{[2 n]}\right)}$ порожден элементами $(38)$ и $Z_{\omega},|\omega|>n$.

СлЕДСтвиЕ 5.6. $\mathcal{D}\left(\mathrm{R}^{[2 n]}\right)=\mathcal{D}(\mathrm{R}) / J_{n}$, где двусторонний идеал $J_{n}$ порожден операторами $D_{\omega},|\omega|>n$.

СлЕДСтвие 5.7. Операторы $d_{2}, d_{3}, d_{4}, \ldots$ алгебраически независимы.

СЛЕДСТВИЕ 5.8. $\operatorname{rk} \mathcal{D}_{-2 n}=c_{n-1}, n \geqslant 1$.

ДокАЗАТЕЛЬство. Пусть $\nu_{n}=\mathrm{rk} \mathbb{Z}\left\langle d_{2}, d_{3}, d_{4}, \ldots\right\rangle_{-2 n}$. Имеем $\nu_{0}=1, \nu_{1}=0$, $\nu_{2}=1, \nu_{3}=1, \nu_{4}=2, \nu_{5}=3, \ldots$. Легко видеть, что при $n \geqslant 1$ выполнено рекуррентное соотношение

$$
\nu_{n+1}=\nu_{n-1}+\nu_{n-2}+\cdots+\nu_{2}+1=\nu_{n-1}+\nu_{n-2}+\cdots+\nu_{2}+\nu_{1}+\nu_{0} .
$$

Поэтому $\nu_{n+1}=\nu_{n}+\nu_{n-1}, n \geqslant 3$. Так как $\nu_{2}=\nu_{3}=1$, то $\nu_{n}=c_{n-2}, n \geqslant 2$.

Из леммы 5.2 следует, что $\operatorname{rk} \mathcal{D}_{-2 n}=\nu_{n}+\nu_{n-1}, n \geqslant 3$. Поэтому $\operatorname{rk} \mathcal{D}_{-2 n}=$ $c_{n-2}+c_{n-3}=c_{n-1}, n \geqslant 3$. Легко видеть, что последняя формула верна и для $n=1,2$.

Напомним (см. утверждение 1.69), что на кольце простых многогранников имеют место соотношения $\left.d_{k}\right|_{\mathcal{P}_{\mathrm{s}}}=\left.\frac{d^{k}}{k !}\right|_{\mathcal{P}_{\mathrm{s}}}$ и кольцо $\mathcal{D}\left(\mathcal{P}_{\mathrm{s}}\right)$ изоморфно алгебре разделенных степеней $\mathcal{Z} / \widehat{J}$, где идеал $\widehat{J}$ порожден соотношениями $Z_{k} Z_{l}-$ $\left(\begin{array}{c}k+l \\ k\end{array}\right) Z_{k+l}$ 
УтВеРЖДЕНИЕ 5.9. Имеет место изоморфизм градуированных колеи:

$$
\mathcal{D} \otimes \mathbb{Q} \rightarrow \mathbb{Q}\left\langle d_{1}, d_{3}, d_{5}, \ldots\right\rangle,
$$

при котором $d_{2 k+1} \rightarrow d_{2 k+1}, k \geqslant 0, u$

$$
d_{2 k} \rightarrow \sum_{i=1}^{k}(-1)^{i-1} \frac{\left(\begin{array}{c}
2 i-2 \\
i-1
\end{array}\right)}{i \cdot 2^{2 i-1}}\left(\sum_{j_{1}+j_{2}+\cdots+j_{2 i}=i+k, j_{l} \geqslant 1} d_{2 j_{1}-1} \ldots d_{2 j_{2 i}-1}\right) .
$$

ДОКАЗАТЕЛЬСТво. Положим

$$
a(t)=\frac{\Phi(t)+\Phi(-t)}{2}=\sum_{k \geqslant 0} d_{2 k} t^{2 k}, \quad b(t)=\frac{\Phi(t)-\Phi(-t)}{2}=\sum_{k \geqslant 0} d_{2 k+1} t^{2 k+1} .
$$

Тогда

$$
\begin{gathered}
a(-t)=a(t), \quad b(-t)=-b(t), \\
\Phi(t)=a(t)+b(t), \quad \Phi(-t)=a(t)-b(t), \\
\Phi(-t) \Phi(t)=\left(a(t)^{2}-b(t)^{2}\right)+(a(t) b(t)-b(t) a(t)) .
\end{gathered}
$$

Так как первое слагаемое в правой части последнего равенства четно, а второе нечетно, то соотношение $\Phi(-t) \Phi(t)=1$ эквивалентно соотношениям

$$
a(t)^{2}-b(t)^{2}=1, \quad a(t) b(t)=b(t) a(t) .
$$

Поэтому $a(t)=\sqrt{1+b(t)^{2}}$ и формула (47) верна. Следовательно, все операторы $d_{2 k}$ выражаются в виде некоммутативных полиномов от операторов $d_{1}, d_{3}, \ldots$ с рациональными коэффициентами. Например, $d_{2}=d^{2} / 2, d_{4}=$ $\left(d d_{3}+d_{3} d\right) / 2-d^{4} / 8$.

Это означает, что алгебра $\mathcal{D} \otimes \mathbb{Q}$ порождается операторами $d_{1}, d_{3}, d_{5}, \ldots$. С другой стороны, найдем число $l_{n}$ мономов вида $d_{2 j_{1}-1} \cdots d_{2 j_{k}-1}$ таких, что $\left(2 j_{1}-1\right)+\cdots+\left(2 j_{k}-1\right)=n$. Положим $l_{0}=1$. Мы имеем:

$$
l_{1}=1, \quad l_{2}=1, \quad l_{3}=2, \quad l_{4}=3, \ldots .
$$

Кроме того, верна рекуррентная формула $l_{n+1}=l_{n}+l_{n-2}+l_{n-4}+\cdots, n \geqslant 0$. Тогда $l_{n+1}=l_{n}+l_{n-1}, n \geqslant 2$, поэтому $l_{n}=c_{n-1}$.

Таким образом, число мономов градуировки $2 n$ равно размерности линейного пространства $\mathcal{D}_{-2 n} \otimes \mathbb{Q}$. Поэтому они линейно независимы. Следовательно, операторы $d_{1}, d_{3}, d_{5}, \ldots$ алгебраически независимы над $\mathbb{Q}$ и $\mathcal{D} \otimes \mathbb{Q}=$ $\mathbb{Q}\left\langle d_{1}, d_{3}, d_{5}, \ldots\right\rangle$.

СлЕДСТвиЕ 5.10. Изоморфизм (46) задает на $\mathbb{Q}\left\langle d_{1}, d_{3}, d_{5}, \ldots\right\rangle$ структуру алгебры Хопфа, содержащей алгебру операторов граней $\mathcal{D}$ в качестве подалгебры Хопфа.

ОПРЕДЕЛЕниЕ 5.11 (см. пример 1.70). Введем операторы $s_{k} \in \mathcal{D} \otimes \mathbb{Q}, k \geqslant 1$, по формуле

$$
s(t)=s_{1} t+s_{2} t^{2}+s_{3} t^{3}+\cdots=\log \Phi(t)=\sum \frac{(-1)^{k-1}}{k}(\Phi(t)-1)^{k}, \quad k \geqslant 1 .
$$

Например, $s_{1}=d, s_{2}=0, s_{3}=d_{3}-d^{3} / 6$. Из соотношения $\Phi(-t) \Phi(t)=1$ мы получаем, что $s(-t)+s(t)=0$. Поэтому $s_{2 k}=0$ для всех $k$.

Из соотношения $\Delta \Phi(t)=\Phi(t) \otimes \Phi(t)=(\Phi(t) \otimes 1) \cdot(1 \otimes \Phi(t))$ мы получаем, что $\Delta s(t)=1 \otimes s(t)+s(t) \otimes 1$. Поэтому каждый оператор $s_{2 k+1}$ является дифференцированием кольца $\mathrm{R} \otimes \mathbb{Q}$. 
УТВЕРЖДЕНИЕ 5.12. Имеет место изоморфизм градуированных алгебр Xonøa:

$$
\mathcal{D} \otimes \mathbb{Q}=\mathbb{Q}\left\langle s_{1}, s_{3}, s_{5}, \ldots\right\rangle,
$$

где $\mathbb{Q}\left\langle s_{1}, s_{3}, s_{5} \ldots\right\rangle$ - свободная ассочиативная алгебра Ли-Хопфа с образующими $s_{2 k-1}, k \geqslant 1$, степени $-2(2 k-1)$.

\section{6. Флаговые полиномы}

6.1. Конструкция. Любое линейное отображение колец $a: R_{1} \rightarrow R_{2}$ можно продолжить до линейного отображения $a: R_{1}[t] \rightarrow R_{2}[t]$, положив $a\left(r_{0}+r_{1} t+\right.$ $\left.\cdots+r_{n} t^{n}\right)=a\left(r_{0}\right)+a\left(r_{1}\right) t+\cdots+a\left(r_{n}\right) t^{n}$. Если $a-$ кольцевой гомоморфизм, то и его продолжение является кольцевым гомоморфизмом.

Согласно утверждению 1.66 , отображение $\Phi(t): \mathrm{R} \rightarrow \mathrm{R}[t]$ является кольцевым гомоморфизмом. Тогда соответствие $P \rightarrow \Phi\left(t_{2}\right) \Phi\left(t_{1}\right) P$ описанным выше способом определяет кольцевой гомоморфизм $\mathrm{R} \rightarrow \mathcal{Q} \operatorname{sym}\left[t_{1}, t_{2}\right] \otimes \mathrm{R} \subset \mathrm{R}\left[t_{1}, t_{2}\right]$.

Итерируя эту конструкцию, на $n$-м шаге мы получим кольцевой гомоморфизм

$$
\Phi_{n}: \mathrm{R} \rightarrow \operatorname{Qsym}\left[t_{1}, \ldots, t_{n}\right] \otimes \mathrm{R}, \quad \Phi_{n}\left(t_{1}, \ldots, t_{n}\right) P=\Phi\left(t_{n}\right) \cdots \Phi\left(t_{1}\right) P .
$$

По построению $\Phi_{n}\left(t_{1}, \ldots, t_{n-1}, 0\right)=\Phi_{n-1}\left(t_{1}, \ldots, t_{n-1}\right)$. Это позволяет получить предельный гомоморфизм $\Phi_{\infty}: \mathrm{R} \rightarrow \mathcal{Q}$ sym $\otimes \mathrm{R}$,

$$
\Phi_{\infty} P=\sum_{k \geqslant 0} \sum_{\left(j_{1}, \ldots, j_{k}\right)} M_{\left(j_{k}, \ldots, j_{1}\right)} \otimes\left(d_{j_{1}} \cdots d_{j_{k}} P\right) .
$$

УТВЕРЖДЕНИЕ 6.1. Любой колъцевой гомоморфизм $\varkappa: \mathrm{R} \rightarrow \mathcal{A}$ индуцирует кольщевой гомоморфизм

$$
\begin{gathered}
\mathcal{F}_{\varkappa}: \mathrm{R} \rightarrow \mathcal{Q} \text { sym } \otimes \mathcal{A}, \\
\mathcal{F}_{\varkappa}(P)=(1 \otimes \varkappa) \Phi_{\infty}(P)=\sum_{k \geqslant 0} \sum_{\left(j_{1}, \ldots, j_{k}\right)} M_{\left(j_{k}, \ldots, j_{1}\right)} \otimes \varkappa\left(d_{j_{1}} \cdots d_{j_{k}} P\right) .
\end{gathered}
$$

Для каждого $r \geqslant 0$ имеем кольцевые гомоморфизмы

$$
\mathcal{F}_{\varkappa, r}=\mathcal{F}_{\varkappa}\left(t_{1}, \ldots, t_{r}, 0,0, \ldots\right): \mathrm{R} \rightarrow \operatorname{Qsym}\left[t_{1}, \ldots, t_{r}\right] \otimes \mathcal{A} .
$$

Согласно замечанию 2.3 , образ $\mathcal{F}_{\varkappa}(P)$ элемента $P \in \mathrm{R}^{2 n}$ полностью определяется полиномом $\mathcal{F}_{\varkappa, r}(P)$ для $r \geqslant n$.

Описанная конструкция приводит к следующим важным гомоморфизмам.

(i) $\alpha$-характер $\xi_{\alpha}: \mathcal{P} \rightarrow \mathbb{Z}[\alpha]$ задает кольцевой гомоморфизм $\mathcal{F}_{\mathcal{P}}=\mathcal{F}_{\xi_{\alpha}}: \mathcal{P} \rightarrow$ $\mathcal{Q} \operatorname{sym}[\alpha]$ :

$$
\mathcal{F}_{\mathcal{P}}\left(P^{n}\right)=\xi_{\alpha} \Phi_{\infty} P^{n}=\alpha^{n}+\sum_{k=1}^{n} \sum_{0 \leqslant a_{1}<\cdots<a_{k} \leqslant n-1} f_{\left\{a_{1}, \ldots, a_{k}\right\}} \alpha^{a_{1}} M_{\left(n-a_{k}, \ldots, a_{2}-a_{1}\right)} .
$$

Для $n=1$ получаем $\mathcal{F}_{\mathcal{P}, 1}\left(\alpha, t_{1}\right)\left(P^{n}\right)=\alpha^{n}+f_{\{n-1\}} \alpha^{n-1} t_{1}+\cdots+f_{\{0\}} t_{1}^{n}-$ однородный $f$-полином от двух переменных ([2], см. также определение 1.27 в настоящем обзоре). Таким образом, полином $\mathcal{F}_{\mathcal{P}, n}, n \geqslant 2$, является обобщением $f$-полинома выпуклого многогранника. 
(ii) $\alpha$-характер $\varepsilon_{\alpha}: \mathcal{P} \rightarrow \mathbb{Z}[\alpha]$ задает кольцевой гомоморфизм $\mathcal{F}_{\mathcal{R} \mathcal{P}}=\mathcal{F}_{\varepsilon_{\alpha}}$ : $\mathcal{R P} \rightarrow \mathcal{Q} \operatorname{sym}[\alpha]:$

$$
\begin{aligned}
& \mathcal{F}_{\mathcal{R P}}\left(P^{n}\right)=\varepsilon_{\alpha} \Phi_{\infty} P^{n}=\left(M_{(n+1)}+\alpha^{n+1}\right) \\
& \quad+\sum_{k=1}^{n} \sum_{0 \leqslant a_{1}<\cdots<a_{k} \leqslant n-1} f_{\left\{a_{1}, \ldots, a_{k}\right\}}\left(M_{\left(n-a_{k}, \ldots, a_{2}-a_{1}, a_{1}+1\right)}+\alpha^{a_{1}+1} M_{\left(n-a_{k}, \ldots, a_{2}-a_{1}\right)}\right) .
\end{aligned}
$$

(iii) Аугментация $\varepsilon: \mathcal{R} \mathcal{P} \rightarrow \mathbb{Z}$ задает кольцевой гомоморфизм $\mathcal{F}_{\varepsilon}: \mathcal{R} \mathcal{P} \rightarrow$ Qsym:

$$
\mathcal{F}_{\varepsilon}\left(P^{n}\right)=\sum_{0 \leqslant a_{1}<\cdots<a_{k} \leqslant n-1} f_{\left\{a_{1}, \ldots, a_{k}\right\}} M_{\left(n-a_{k}, \ldots, a_{2}-a_{1}, a_{1}+1\right)} .
$$

При этом $\mathcal{F}_{\varepsilon}(P)=* \mathrm{~F}(L(P))$, где $*$ - инволюция на алгебре Хопфа $\mathcal{Q} s y m, L-$ вложение $\mathcal{R} \mathcal{P} \rightarrow \mathcal{R}$, а $\mathrm{F}$ - отображение Эренборга $\mathcal{R} \rightarrow \mathcal{Q}$ sym.

ЗАмЕчАниЕ 6.2. Мы будем считать, что отображение $\mathcal{F}_{\mathcal{P}}$ определено и на кольце $\mathcal{R} \mathcal{P}$, причем $\mathcal{F}_{\mathcal{P}}(\varnothing)=0$.

УтВЕРЖДЕНИЕ 6.3. На колъце $\mathcal{R} \mathcal{P}$ имеем:

$$
\mathcal{F}_{\mathcal{P}}=\partial \mathcal{F}_{\varepsilon}, \quad \mathcal{F}_{\mathcal{R} \mathcal{P}}(P)=\mathcal{F}_{\varepsilon}(P)+\alpha \mathcal{F}_{\mathcal{P}}(P) .
$$

СлеДСТвИЕ 6.4. Для любих двух элементов $P, Q \in \mathcal{R} \mathcal{P}$ мы имеем:

$$
\mathcal{F}_{\mathcal{P}}(P * Q)=\mathcal{F}_{\mathcal{P}}(P) \cdot \mathcal{F}_{\varepsilon}(Q)+\mathcal{F}_{\varepsilon}(P) \cdot \mathcal{F}_{\mathcal{P}}(Q)+\alpha \mathcal{F}_{\mathcal{P}}(P) \cdot \mathcal{F}_{\mathcal{P}}(Q),
$$

m.е. отображение $\mathcal{F}_{\mathcal{P}}: \mathcal{R P} \rightarrow \mathcal{Q} \operatorname{sym}[\alpha]$ является $\mathcal{F}_{\varepsilon}-\alpha$-дифферениированием.

СлЕДСТвИЕ 6.5. $\mathcal{F}_{\mathcal{P}}(C P)=\mathcal{F}_{\varepsilon}(P)+\left(\alpha+\sigma_{1}\right) \mathcal{F}_{\mathcal{P}}(P)$ для любого $P \in \mathcal{R} \mathcal{P}$.

УтВЕРЖДЕНИЕ 6.6. Соответствие $\left.\mathcal{F}_{\mathcal{R P}} \rightarrow \mathcal{F}_{\mathcal{R P}}\right|_{\alpha=0}=\mathcal{F}_{\varepsilon}$ задает изоморфизм $\mathcal{F}_{\mathcal{R} \mathcal{P}}(\mathcal{R P}) \rightarrow \mathcal{F}_{\varepsilon}(\mathcal{R P})$.

ДокАЗАтЕЛьСтво. Ясно, что это рассматриваемое соответствие задает эпиморфизм, который является мономорфизмом, так как из условия $\mathcal{F}_{\varepsilon}(P)=0$ вытекает, что все флаговые числа элемента $P \in \mathcal{R} \mathcal{P}$ равны нулю, а следовательно, $\mathcal{F}_{\mathcal{R P}}(P)=0$.

6.2. Кольца флаговых векторов. Для каждого из отображений $\mathcal{F}_{\mathcal{P}}$, $\mathcal{F}_{\mathcal{R}} \mathcal{P}$ и $\mathcal{F}_{\varepsilon}$ образы двух элементов $P, Q \in \mathrm{R}$ совпадают тогда и только тогда, когда они имеют одинаковые флаговые векторы.

ОПРЕДЕЛЕНИЕ 6.7. Назовем $\times$-кольцом флаговых векторов выпуклых многогранников кольцо $\left(\mathcal{P} / \operatorname{Ker} \mathcal{F}_{\mathcal{P}}\right) \simeq \mathcal{F}_{\mathcal{P}}(\mathcal{P})$, а *-кольцом флаговых векторов кольцо $\left(\mathcal{R P} / \operatorname{Ker} \mathcal{F}_{\mathcal{R} \mathcal{P}}\right)=\left(\mathcal{R} \mathcal{P} / \operatorname{Ker} \mathcal{F}_{\varepsilon}\right) \simeq \mathcal{F}_{\varepsilon}(\mathcal{R} \mathcal{P}) \simeq \mathcal{F}_{\mathcal{R} \mathcal{P}}(\mathcal{R} \mathcal{P})$.

Теорема 6.8. Подгруппа $\mathcal{F}_{\mathcal{P}, r}\left(\mathcal{P}^{2 n}\right) \subset \operatorname{Qsym}\left[t_{1}, \ldots, t_{r}\right][\alpha], r \geqslant n$, является прямым слагаемым и состоит из всех однородных полиномов $g$ степени $2 n$ maкux, что

$$
\begin{aligned}
& g\left(\alpha, t_{1},-t_{1}, t_{3}, \ldots, t_{r}\right)=g\left(\alpha, 0,0, t_{3}, \ldots, t_{r}\right), \\
& g\left(\alpha, t_{1}, t_{2},-t_{2}, t_{4}, \ldots, t_{r}\right)=g\left(\alpha, t_{1}, 0,0, t_{4} \ldots, t_{r}\right), \\
& \begin{array}{l}
\ldots\left(\alpha, t_{1}, \ldots, t_{r-2}, t_{r-1},-t_{r-1}\right)=g\left(\alpha, t_{1}, \ldots, t_{r-2}, 0,0\right), \\
\text {, }
\end{array}
\end{aligned}
$$


$u$

$$
g\left(-\alpha, t_{1}, \ldots, t_{r-1}, \alpha\right)=g\left(\alpha, t_{1}, \ldots, t_{r-1}, 0\right)
$$

Эти уравнения равносильны соотношениям Байер-Биллера (26).

ДокАЗАтельство. Тот факт, что подгруппа $\mathcal{F}_{\mathcal{P}, r}\left(\mathcal{P}^{2 n}\right)$ является прямым слагаемым, непосредственно вытекает из уравнений (53) и (54), поэтому достаточно показать, что она описывается этими уравнениями. Мы имеем: $\Phi(-t) \Phi(t)=1=\Phi(0) \Phi(0)$, поэтому

$$
\begin{aligned}
& \xi_{\alpha} \Phi\left(t_{r}\right) \cdots \Phi\left(t_{3}\right) \Phi\left(-t_{1}\right) \Phi\left(t_{1}\right) P^{n}=\xi_{\alpha} \Phi\left(t_{r}\right) \cdots \Phi\left(t_{3}\right) \Phi(0) \Phi(0) P^{n}, \\
& \xi_{\alpha} \Phi\left(t_{r}\right) \cdots \Phi\left(t_{4}\right) \Phi\left(-t_{2}\right) \Phi\left(t_{2}\right) \Phi\left(t_{1}\right) P^{n}=\xi_{\alpha} \Phi\left(t_{r}\right) \cdots \Phi\left(t_{4}\right) \Phi(0) \Phi(0) \Phi\left(t_{1}\right) P^{n} \text {, } \\
& \xi_{\alpha} \Phi\left(-t_{r-1}\right) \Phi\left(t_{r-1}\right) \Phi\left(t_{n-2}\right) \cdots \Phi\left(t_{1}\right) P^{n}=\xi_{\alpha} \Phi(0) \Phi(0) \Phi\left(t_{r-2}\right) \cdots \Phi\left(t_{1}\right) P^{n} .
\end{aligned}
$$

Равенство

$$
\xi_{-\alpha} \Phi(\alpha) \Phi\left(t_{r-1}\right) \cdots \Phi\left(t_{1}\right) P^{n}=\xi_{\alpha} \Phi(0) \Phi\left(t_{r-1}\right) \cdots \Phi\left(t_{1}\right) P^{n}
$$

вытекает из утверждения 1.68. Докажем теперь, что если однородный полином $g$ степени $2 n$ удовлетворяет условиям $(53)$ и $(54)$, то $g=\mathcal{F}_{\mathcal{P}, r}(P)$ для некоторого элемента $P \in \mathcal{P}^{2 n}$.

Любую функцию $g \in \operatorname{Qsym}\left[t_{1}, \ldots, t_{r}\right][\alpha]$ степени $2 n$ можно записать в виде

$$
g\left(\alpha, t_{1}, \ldots, t_{r}\right)=\sum_{S: l(S) \leqslant r} g_{S} \alpha^{a_{1}} M_{\omega(S)}, \quad S \subseteq[0, n-1] .
$$

В частности, $\mathcal{F}_{\mathcal{P}, r}\left(P^{n}\right)=\sum_{S: l(S) \leqslant r} f_{S} \alpha^{a_{1}} M_{\omega(S)}$.

Лемма 6.9. Пусть $g \in \mathrm{Qsym}\left[t_{1}, \ldots, t_{r}\right][\alpha]$ - однородный полином степени 2n. Тогда:

1) уравнения

$$
g\left(\alpha, t_{1}, \ldots, t_{q},-t_{q}, \ldots, t_{r}\right)=g\left(\alpha, t_{1}, \ldots, 0,0, \ldots, t_{r}\right)
$$

равносильны соотношениям

$$
\sum_{j=a_{t}+1}^{a_{t+1}-1}(-1)^{j-a_{t}-1} g_{\left\{a_{1}, \ldots, a_{t}, j, a_{t+1}, \ldots, a_{k}\right\}}=\left(1+(-1)^{a_{t+1}-a_{t}}\right) g_{\left\{a_{1}, \ldots, a_{t}, a_{t+1}, \ldots, a_{k}\right\}}
$$

для $1 \leqslant k \leqslant \min \{r-1, n-1\}, k+1-q \leqslant t \leqslant r-q$

2) уравнение

$$
g\left(-\alpha, t_{1}, \ldots, t_{r-1}, \alpha\right)=g\left(\alpha, t_{1}, \ldots, t_{r-1}, 0\right)
$$

равносильно соотношениям

$$
\sum_{j=0}^{a_{1}-1}(-1)^{j} g_{\left\{j, a_{1}, \ldots, a_{k}\right\}}=\left(1+(-1)^{a_{1}-1}\right) g_{\left\{a_{1}, \ldots, a_{k}\right\}} \quad \text { для } 0 \leqslant k \leqslant \min \{r-1, n-1\} .
$$


ДокАЗАТЕЛЬСтво. Имеем:

$$
\begin{aligned}
& g\left(\alpha, t_{1}, \ldots, t_{q},-t_{q}, \ldots, t_{r}\right) \\
& =g_{\varnothing} \alpha^{n}+\sum_{k=1}^{\min \{r, n\}} \sum_{0 \leqslant a_{1}<\cdots<a_{k} \leqslant n-1} g_{\left\{a_{1}, \ldots, a_{k}\right\}} \alpha^{a_{1}} \\
& \times\left.\left(\sum_{1 \leqslant l_{1}<\cdots<l_{k} \leqslant r} t_{l_{1}}^{n-a_{k}} \cdots t_{l_{k}}^{a_{2}-a_{1}}\right)\right|_{t_{q+1}=-t_{q}} \\
& \min \{r-2, n\} \\
& =g_{\varnothing} \alpha^{n}+\sum_{k=1} \sum_{0 \leqslant a_{1}<\cdots<a_{k} \leqslant n-1} g_{\left\{a_{1}, \ldots, a_{k}\right\}} \alpha^{a_{1}} \\
& \times\left(\sum_{l_{j} \neq q, q+1} t_{l_{1}}^{n-a_{k}} \cdots t_{l_{k}}^{a_{2}-a_{1}}\right) \\
& \min \{r-1, n\} \\
& +\sum_{k=1} \sum_{0 \leqslant a_{1}<\cdots<a_{k} \leqslant n-1} g_{\left\{a_{1}, \ldots, a_{k}\right\}} \alpha^{a_{1}}
\end{aligned}
$$

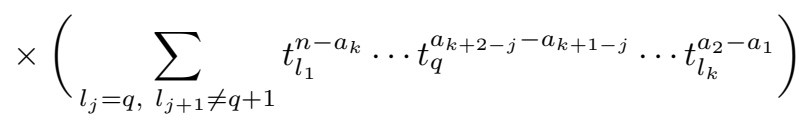

$$
\begin{aligned}
& +\sum_{k=1}^{\min \{r-1, n\}} \sum_{0 \leqslant a_{1}<\cdots<a_{k} \leqslant n-1} g_{\left\{a_{1}, \ldots, a_{k}\right\}} \alpha^{a_{1}} \\
& \times\left(\sum_{l_{j} \neq q,} t_{l_{j+1}=q+1}^{n-a_{k}} \cdots\left(-t_{q}\right)^{a_{k+1-j}-a_{k-j}} \cdots t_{l_{k}}^{a_{2}-a_{1}}\right) \\
& +\sum_{k=1}^{\min \{r, n\}} \sum_{0 \leqslant a_{1}<\cdots<a_{k} \leqslant n-1} g_{\left\{a_{1}, \ldots, a_{k}\right\}} \alpha^{a_{1}} \\
& \times\left(\sum_{l_{j}=q,} t_{l_{j+1}=q+1}^{n-a_{k}} \cdots(-1)^{a_{k+1-j}-a_{k-j}} t_{q}^{a_{k+2-j}-a_{k-j}} \cdots t_{l_{k}}^{a_{2}-a_{1}}\right) .
\end{aligned}
$$

Первые два слагаемых образуют в точности $g\left(\alpha, t_{1}, \ldots, 0,0, \ldots, t_{r}\right)$. Поэтому все коэффициенты полинома, состоящего из последних трех слагаемых, должны быть равны нулю.

Рассмотрим моном

$$
\alpha^{a_{1}} t_{l_{1}}^{n-a_{k}} \cdots t_{q}^{a_{t+1}-a_{t}} \cdots t_{l_{k}}^{a_{2}-a_{1}}, \quad \text { где } q=l_{k+1-t} .
$$

Наличие такого монома в сумме эквивалентно условиям

$$
k \leqslant \min \{r-1, n-1\}, \quad k+1-t \leqslant q, \quad t-1 \leqslant r-q-1 .
$$

Равенство нулю коэффициента при таком мономе равносильно соотношению

$$
\left(1+(-1)^{a_{t+1}-a_{t}}\right) g_{\left\{a_{1}, \ldots, a_{t}, a_{t+1}, \ldots, a_{k}\right\}}+\sum_{j=a_{t}+1}^{a_{t+1}-1}(-1)^{j-a_{t}} g_{\left\{a_{1}, \ldots, a_{t}, j, a_{t+1}, a_{k}\right\}}=0 .
$$

Таким образом, мы доказали первую часть леммы. 
Из подробной записи уравнения $g\left(-\alpha, t_{1}, \ldots, t_{r-1}, \alpha\right)=g\left(\alpha, t_{1}, \ldots, t_{r-1}, 0\right)$ :

$$
\begin{aligned}
& g_{\varnothing}(-\alpha)^{n}+\sum_{k=1}^{\min \{r-1, n\}} \sum_{0 \leqslant a_{1}<\cdots<a_{k} \leqslant n-1} g_{\left\{a_{1}, \ldots, a_{k}\right\}}(-\alpha)^{a_{1}} \\
& \times\left(\sum_{1 \leqslant l_{1}<\cdots<l_{k} \leqslant r-1} t_{l_{1}}^{n-a_{k}} \cdots t_{l_{k}}^{a_{2}-a_{1}}\right) \\
& +\sum_{k=1}^{\min \{r, n\}} \sum_{0 \leqslant a_{1}<\cdots<a_{k} \leqslant n-1} g_{\left\{a_{1}, \ldots, a_{k}\right\}}(-\alpha)^{a_{1}} \\
& \times\left(\sum_{1 \leqslant l_{1}<\cdots<l_{k}=r} t_{l_{1}}^{n-a_{k}} \cdots t_{l_{k-1}}^{a_{3}-a_{2}} \alpha^{a_{2}-a_{1}}\right) \\
& =g_{\varnothing} \alpha^{n}+\sum_{k=1}^{\min \{r-1, n\}} \sum_{0 \leqslant a_{1}<\cdots<a_{k} \leqslant n-1} g_{\left\{a_{1}, \ldots, a_{k}\right\}} \alpha^{a_{1}} \\
& \times\left(\sum_{1 \leqslant l_{1}<\cdots<l_{k} \leqslant r-1} t_{l_{1}}^{n-a_{k}} \cdots t_{l_{k}}^{a_{2}-a_{1}}\right)
\end{aligned}
$$

следует, что оно равносильно соотношениям

$$
(-1)^{a_{1}} g_{\left\{a_{1}, \ldots, a_{k}\right\}}+\sum_{j=0}^{a_{1}-1}(-1)^{j} g_{\left\{j, a_{1}, \ldots, a_{k}\right\}}=g_{\left\{a_{1}, \ldots, a_{k}\right\}}
$$

для $0 \leqslant k \leqslant \min \{r-1, n-1\}$.

В частности, при $k=0$ мы имеем формулу Эйлера-Пуанкаре

$$
(-1)^{n} g_{\varnothing}+(-1)^{n-1} g_{\{n-1\}}+\cdots+g_{\{2\}}-g_{\{1\}}+g_{\{0\}}=g_{\varnothing} .
$$

Это завершает доказательство леммы.

СлЕДСтвиЕ 6.10. Для однородного полинома $g \in \operatorname{Qsym}\left[t_{1}, \ldots, t_{r}\right][\alpha]$ cmeneни 2 п при $r \geqslant n$ имеем:

- функциональные уравнения (53) равносильны соотношениям Байер-Биллера (26), $i \neq-1$, на коэфбичиенты $\left\{g_{S}, S \subseteq\{0,1, \ldots, n-1\}\right\}$,

- функциональное уравнение (54) равносильно соотношениям Байер-Биллеpa (26), $i=-1$, на коэффичиенты $\left\{g_{S}, S \subseteq\{0,1, \ldots, n-1\}\right\}$.

ДокАЗАТЕЛЬСтво. Мы видим, что соотношения (53) и (54) следуют из соотношений Байер-Биллера для $i \neq-1$ и $i=-1$ соответственно.

С другой стороны, для $i=-1$ соотношения (26) следуют из уравнения (54).

Если же $S=\left\{a_{1}, \ldots, a_{t}, a_{t+1}, \ldots, a_{s}\right\}, i=a_{t} \geqslant 0$ и $k=a_{t+1}$ или если $S=$ $\left\{a_{1}, \ldots, a_{t}\right\}, i=a_{t} \geqslant 0, s=t$ и $k=n$, то мы можем в лемме 6.9 взять любое число $q$ такое, что $s+1-t \leqslant q \leqslant r-t$.

Теперь закончим доказательство теоремы 6.8. Если однородный полином $g \in \operatorname{Qsym}\left[t_{1}, \ldots, t_{r}\right][\alpha]$ степени $2 n$ удовлетворяет уравнениям (53) и (54), то по следствию 6.10 его коэффициенты $\left\{g_{S}, S \subseteq\{0, \ldots, n-1\}\right\}$ удовлетворяют соотношениям Байер-Биллера. Поэтому согласно следствию 1.86 полином $g$ 
единственным образом представляется в виде целочисленной линейной комбинации $g=\sum_{Q \in \Omega^{n}} \lambda_{Q} \mathcal{F}_{\mathcal{P}, r}(Q)$. Тогда

$$
g=\sum_{Q \in \Omega^{n}} \lambda_{Q} \mathcal{F}_{\mathcal{P}, r}(Q)=\mathcal{F}_{\mathcal{P}, r}\left(\sum_{Q \in \Omega^{n}} \lambda_{Q} Q\right)
$$

Теорема 6.8 доказана.

Из доказательства теоремы 6.8 мы получаем, что $\mathrm{rk} \mathcal{F}_{\mathcal{P}, r}\left(\mathcal{P}^{2 n}\right)=c_{n}, r \geqslant n$.

УтВЕРЖДЕНИЕ 6.11. Пусть $r \geqslant 2$ u $P^{n}-n$-мерный многогранник. Тогда

$$
\mathcal{F}_{\mathcal{P}, r}\left(\alpha, t_{1}, \ldots, t_{r}\right)\left(P^{n}\right)=\mathcal{F}_{\mathcal{P}, 1}\left(\alpha, t_{1}+\cdots+t_{r}\right)\left(P^{n}\right)
$$

в том и только том случае, когда многогранник $P^{n}$ простой.

Напомним, что $\mathcal{F}_{\mathcal{P}, 1}(\alpha, t)=f(\alpha, t)$ есть $f$-полином выпуклого многогранника.

ДокАзАтельство. Согласно утверждению 1.69, на кольце простых многогранников выполнены соотношения $\left.d_{k}\right|_{\mathcal{P}_{\mathrm{s}}}=\left.\frac{1}{k !} d^{k}\right|_{\mathcal{P}_{\mathrm{s}}}$ и $\Phi(t)=e^{t d}$. Поэтому $\Phi\left(t_{r}\right) \Phi\left(t_{r-1}\right) \cdots \Phi\left(t_{1}\right)=\Phi\left(t_{1}+\cdots+t_{r}\right)$, следовательно, $\mathcal{F}_{\mathcal{P}, r}\left(\alpha, t_{1}, \ldots, t_{r}\right)\left(P^{n}\right)=$ $\mathcal{F}_{\mathcal{P}, 1}\left(\alpha, t_{1}+\cdots+t_{r}\right)\left(P^{n}\right)$.

С другой стороны, пусть $\mathcal{F}_{\mathcal{P}, r}\left(\alpha, t_{1}, \ldots, t_{r}\right)\left(P^{n}\right)=\mathcal{F}_{\mathcal{P}, 1}\left(\alpha, t_{1}+\cdots+t_{r}\right)\left(P^{n}\right)$. Тогда $\mathcal{F}_{\mathcal{P}, 2}\left(\alpha, t_{1}, t_{2}\right)\left(P^{n}\right)=\mathcal{F}_{\mathcal{P}, 1}\left(\alpha, t_{1}+t_{2}\right)\left(P^{n}\right)$, т. е.

$\alpha^{n}+\sum_{i=0}^{n-1} f_{\{i\}} \alpha^{i}\left(t_{1}^{n-i}+t_{2}^{n-i}\right)+\sum_{0 \leqslant i<j \leqslant n-1} f_{\{i, j\}} \alpha^{i} t_{1}^{n-j} t_{2}^{j-i}=\alpha^{n}+\sum_{i=0}^{n-1} f_{\{i\}} \alpha^{i}\left(t_{1}+t_{2}\right)^{n-i}$.

В частности, коэффициенты при мономе $t_{1} t_{2}^{n-1}$ слева и справа совпадают. Тогда $f_{\{0, n-1\}}=n f_{\{0\}}$. Следовательно, каждая вершина многогранника $P^{n}$ лежит ровно в $n$ гипергранях, поэтому многогранник $P^{n}$ простой.

ЗАмЕчАниЕ 6.12. Устремляя $r$ к бесконечности, мы получаем, что

$$
\mathcal{F}_{\mathcal{P}}\left(\alpha, t_{1}, t_{2}, \ldots\right)\left(P^{n}\right)=\mathcal{F}_{\mathcal{P}, 1}\left(\alpha, t_{1}+t_{2}+\cdots\right)\left(P^{n}\right)
$$

тогда и только тогда, когда многогранник $P^{n}$ простой.

В случае простых многогранников уравнения (53) тривиальны, а уравнение (54) принимает вид

$$
\mathcal{F}_{\mathcal{P}, 1}\left(-\alpha, t_{1}+\cdots+t_{r-1}+\alpha\right)=\mathcal{F}_{\mathcal{P}, 1}\left(\alpha, t_{1}+\cdots+t_{r-1}\right) .
$$

Положим $t=t_{1}+\cdots+t_{r-1}$. Тогда $\mathcal{F}_{\mathcal{P}, 1}(-\alpha, t+\alpha)=\mathcal{F}_{\mathcal{P}, 1}(\alpha, t)$. Это уравнение равносильно соотношениям Дена-Соммервилля для простых многогранников. (После замены переменных $\mathcal{F}_{\mathcal{P}, 1}(\alpha-t, t)=h(\alpha, t)$ уравнение принимает вид $h(\alpha, t)=h(t, \alpha)$, где $h(\alpha, t)-h$-полином простого многогранника.)

Tеорема 6.13. (i) Подгрупnа $\mathcal{F}_{\mathcal{R} \mathcal{P}, r}\left(\mathcal{R P}^{2 n}\right) \subset \operatorname{Qsym}\left[t_{1}, \ldots, t_{r}\right][\alpha], r \geqslant n, \boldsymbol{я ⿻ -}$ ляется прямым слагаемым и состоит из всех однородных полиномов $g$ степени 2 п таких, что

1) выполнены уравнения (53);

2) выполнено уравнение

$$
g\left(-\alpha, t_{1}, t_{2}, \ldots, t_{r-1}, \alpha\right)=g\left(0, t_{1}, t_{2}, \ldots, t_{r-1}, 0\right) .
$$


(ii) Подгруппа $\mathcal{F}_{\varepsilon, r}\left(\mathcal{R P}^{2 n}\right) \subset \mathrm{Qsym}\left[t_{1}, \ldots, t_{r}\right], r \geqslant n$, является прямым слагаемым и состоит из всех однородных полиномов $g$ степени $2 n$ таких, что выполнены уравнения

$$
\begin{aligned}
& g\left(t_{1},-t_{1}, t_{3}, \ldots, t_{r}\right)=g\left(0,0, t_{3}, \ldots, t_{r}\right) ; \\
& g\left(t_{1}, t_{2},-t_{2}, t_{4}, \ldots, t_{r}\right)=g\left(t_{1}, 0,0, t_{4}, \ldots, t_{r}\right) ;
\end{aligned}
$$

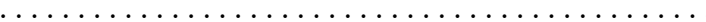

$$
\begin{aligned}
& g\left(t_{1}, \ldots, t_{r-2}, t_{r-1},-t_{r-1}\right)=g\left(t_{1}, \ldots, t_{r-2}, 0,0\right) .
\end{aligned}
$$

В обоих случаях уравнения равносильны соотношениям Байер-Биллера (26).

ДокАЗАТЕЛЬСтво. Так как

$$
\begin{aligned}
\mathcal{F}_{\mathcal{R} \mathcal{P}, r}\left(\alpha, t_{1}, \ldots, t_{r}\right)(P) & =\varepsilon_{\alpha} \Phi\left(t_{r}\right) \cdots \Phi\left(t_{1}\right) P, \\
\mathcal{F}_{\varepsilon, r}\left(t_{1}, \ldots, t_{r}\right)(P) & =\varepsilon_{0} \Phi\left(t_{r}\right) \cdots \Phi\left(t_{1}\right) P=\mathcal{F}_{\mathcal{R} \mathcal{P}, r}\left(0, t_{1}, \ldots, t_{r}\right)(P),
\end{aligned}
$$

то согласно утверждениям 1.67 и 1.68 для любого элемента $P \in \mathcal{R} \mathcal{P}$ полином $\mathcal{F}_{\mathcal{R} \mathcal{P}, r}(P)$ удовлетворяет уравнениям $(53)$ и $(57)$, а полином $\mathcal{F}_{\varepsilon, r}(P)$ удовлетворяет уравнениям (58).

В обратную сторону доказательство аналогично доказательству теоремы 6.8 .

ЗАмечАниЕ 6.14. Отметим, что $\operatorname{rk} \mathcal{F}_{\mathcal{P}}\left(\mathcal{P}^{2 n}\right)=c_{n}=\operatorname{rk} \mathcal{F}_{\varepsilon}\left(\mathcal{R P}^{2(n+1)}\right)$. Это связано с тем, что градуировка $n$-мерного многогранника $P^{n}$ в кольце $\mathcal{P}$ равна $2 n$, а в кольце $\mathcal{R} \mathcal{P}$ равна $2(n+1)$.

ЗАмечАниЕ 6.15. Проекция абелевых групп $\Pi_{\mathcal{R P}}: \mathcal{R} \mathcal{P} \rightarrow \mathcal{P}$, переводящая непустой многогранник в себя и пустое множество $\varnothing$ в нуль, индуцирует проекцию $\Pi_{\mathcal{R} \mathcal{P}}:\left(\mathcal{R} \mathcal{P} / \operatorname{Ker} \mathcal{F}_{\varepsilon}\right) \rightarrow\left(\mathcal{P} / \operatorname{Ker} \mathcal{F}_{\mathcal{P}}\right)$. При отождествлении $\mathcal{P} / \operatorname{Ker} \mathcal{F}_{\mathcal{P}}$ с $\mathcal{F}_{\mathcal{P}}(\mathcal{P})$ проекция П $\mathcal{R} \mathcal{P}$ задается отображением $\mathcal{F}_{\mathcal{P}}$. Если при этом отождествить кольцо $\mathcal{R} \mathcal{P} / \operatorname{Ker} \mathcal{F}_{\varepsilon}$ с $\mathcal{F}_{\varepsilon}(\mathcal{R} \mathcal{P})$, то $\Pi_{\mathcal{R} \mathcal{P}}=\partial$.

6.3. Характеризация флагового полинома $\mathcal{F}_{\mathcal{P}}$. В этом пункте мы найдем условие, которое однозначно определяет флаговый полином $\mathcal{F}_{\mathcal{P}}$.

Сначала установим связь между полиномами $\mathcal{F}_{\mathcal{P}}\left(P^{n}\right)$ и $\mathcal{F}_{\mathcal{P}}\left(d_{k} P^{n}\right)$.

УТВЕРЖДЕНИЕ 6.16. Для многогранника $P^{n}$ выполнено соотношение

$$
\mathcal{F}_{\mathcal{P}}\left(\alpha, t_{1}, t_{2}, \ldots\right)\left(d_{k} P^{n}\right)=\left.\frac{1}{k !} \frac{\partial^{k}}{\partial t^{k}}\right|_{t=0} \mathcal{F}_{\mathcal{P}}\left(\alpha, t, t_{1}, t_{2}, \ldots\right)\left(P^{n}\right) .
$$

ДокАЗАТЕЛЬСтво. Оба выражения принадлежат кольцу $\mathcal{Q} s y m[\alpha]$ и имеют степень $2(n-k)$, поэтому они однозначно определяются своими ограничениями на $n-k$ переменных $t_{1}, \ldots, t_{n-k}$. Мы имеем:

$$
\begin{aligned}
\left.\left(\left.\frac{1}{k !} \frac{\partial^{k}}{\partial t^{k}}\right|_{t=0} \mathcal{F}_{\mathcal{P}}\left(\alpha, t, t_{1}, t_{2}, \ldots\right)\left(P^{n}\right)\right)\right|_{t_{n-k+1}=t_{n-k+2}=\cdots=0} \\
\quad=\left.\frac{1}{k !} \frac{\partial^{k}}{\partial t^{k}}\right|_{t=0} \mathcal{F}_{\mathcal{P}, n-k+1}\left(\alpha, t, t_{1}, t_{2}, \ldots, t_{n-k}\right)\left(P^{n}\right) \\
\quad=\left.\frac{1}{k !} \frac{\partial^{k}}{\partial t^{k}}\right|_{t=0} \xi_{\alpha} \Phi\left(t_{n-k}\right) \cdots \Phi\left(t_{1}\right) \Phi(t) P^{n} \\
\quad=\xi_{\alpha} \Phi\left(t_{n-k}\right) \cdots \Phi\left(t_{1}\right) d_{k} P^{n}=\mathcal{F}_{\mathcal{P}, n-k}\left(\alpha, t_{1}, t_{2}, \ldots, t_{n-k}\right)\left(d_{k} P^{n}\right) \\
\quad=\left.\left(\mathcal{F}_{\mathcal{P}}\left(\alpha, t_{1}, t_{2}, \ldots\right)\left(d_{k} P^{n}\right)\right)\right|_{t_{n-k+1}=t_{n-k+2}=\cdots=0},
\end{aligned}
$$

что и доказывает (59). 
СлЕДСТВИЕ 6.17. Пусть $P^{n}-n$-мерный многогранник. Тогда

$$
\begin{aligned}
& \mathcal{F}_{\mathcal{P}}\left(\alpha, t, t_{1}, t_{2}, \ldots\right)\left(P^{n}\right)=\mathcal{F}_{\mathcal{P}}\left(\alpha, t_{1}, t_{2}, \ldots\right)\left(P^{n}\right)+\mathcal{F}_{\mathcal{P}}\left(\alpha, t_{1}, t_{2}, \ldots\right)\left(d P^{n}\right) t \\
& \quad+\mathcal{F}_{\mathcal{P}}\left(\alpha, t_{1}, t_{2}, \ldots\right)\left(d_{2} P^{n}\right) t^{2}+\cdots+\mathcal{F}_{\mathcal{P}}\left(\alpha, t_{1}, t_{2}, \ldots\right)\left(d_{n} P^{n}\right) t^{n} \\
& \mathcal{F}_{\mathcal{P}, r+1}\left(\alpha, t, t_{1}, \ldots, t_{r}\right)\left(P^{n}\right)=\mathcal{F}_{\mathcal{P}, r}\left(\alpha, t_{1}, \ldots, t_{r}\right)\left(P^{n}\right) \\
& \quad+\mathcal{F}_{\mathcal{P}, r}\left(\alpha, t_{1}, \ldots, t_{r}\right)\left(d P^{n}\right) t+\mathcal{F}_{\mathcal{P}, r}\left(\alpha, t_{1}, \ldots, t_{r}\right)\left(d_{2} P^{n}\right) t^{2}+\cdots \\
& \quad+\mathcal{F}_{\mathcal{P}, r}\left(\alpha, t_{1}, \ldots, t_{r}\right)\left(d_{n} P^{n}\right) t^{n}, \quad r \geqslant 0 .
\end{aligned}
$$

ДокАзАТЕЛЬство. Первое равенство следует из утверждения 6.16. Для $r \geqslant 0$ имеем:

$$
\begin{aligned}
\mathcal{F}_{\mathcal{P}, r+1}\left(\alpha, t, t_{1}, \ldots, t_{r}\right)\left(P^{n}\right)=\xi_{\alpha} \Phi\left(t_{r}\right) & \cdots \Phi\left(t_{1}\right) \Phi(t) P^{n} \\
= & \sum_{k=0}^{n}\left(\xi_{\alpha} \Phi\left(t_{r}\right) \cdots \Phi\left(t_{1}\right) d_{k} P^{n}\right) t^{k}=\sum_{k=0}^{n} \mathcal{F}_{\mathcal{P}, r}\left(\alpha, t_{1}, \ldots, t_{r}\right)\left(d_{k} P^{n}\right) t^{k},
\end{aligned}
$$

что и доказывает равенство (61).

Равенство (60) эквивалентно условию

$$
\mathcal{F}_{\mathcal{P}}\left(\alpha, t_{1}, t_{2}, \ldots\right)\left(\Phi(t) P^{n}\right)=\mathcal{F}_{\mathcal{P}}\left(\alpha, t, t_{1}, t_{2}, \ldots\right)\left(P^{n}\right),
$$

т. е. коммутативности диаграммы

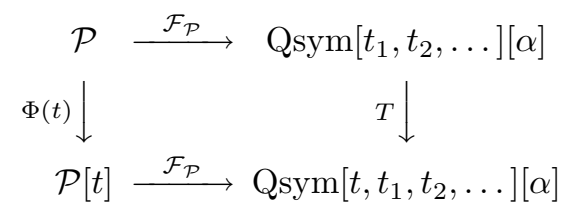

где $T: \operatorname{Qsym}\left[t_{1}, t_{2}, \ldots\right][\alpha] \rightarrow \operatorname{Qsym}\left[t, t_{1}, t_{2}, \ldots\right][\alpha]$ - кольцевой гомоморфизм:

$$
T g\left(\alpha, t_{1}, t_{2}, \ldots\right)=g\left(\alpha, t, t_{1}, t_{2}, \ldots\right), \quad g \in \operatorname{Qsym}\left[t_{1}, t_{2}, \ldots\right][\alpha] .
$$

ЗАМЕЧАНИЕ 6.18. Для всех $r \geqslant 0$ имеем

$$
\mathcal{F}_{\mathcal{P}, r}\left(\alpha, t_{1}, \ldots, t_{r}\right)\left(\Phi(t) P^{n}\right)=\mathcal{F}_{\mathcal{P}, r+1}\left(\alpha, t, t_{1}, \ldots, t_{r}\right)\left(P^{n}\right) .
$$

Рассмотрим гомоморфизм $T_{r+1}: \operatorname{Qsym}\left[t_{1}, \ldots, t_{r}\right][\alpha] \rightarrow \operatorname{Qsym}\left[t, t_{1}, \ldots, t_{r}\right][\alpha]$ :

$$
T_{r+1}(\alpha)=\alpha, \quad T_{r+1} M_{\omega}\left(t_{1}, \ldots, t_{r}\right)=M_{\omega}\left(t, t_{1}, \ldots, t_{r}\right) .
$$

Тогда равенство $\mathcal{F}_{\mathcal{P}, r}\left(\alpha, t_{1}, \ldots, t_{r}\right)\left(\Phi(t) P^{n}\right)=\left(T_{r+1} \mathcal{F}_{\mathcal{P}, r}\right)\left(\alpha, t, t_{1}, \ldots, t_{r}\right)$ верно только при $r \geqslant n$.

ТеОрема 6.19. Пусть $\psi: \mathcal{P} \rightarrow \mathcal{Q}$ sym $[\alpha]$ - гомоморфизм абелевых групп такой, чmo

1) $\psi(\alpha, 0,0, \ldots)\left(P^{n}\right)=\alpha^{n}$ для любого $n$-мерного многогранника $P^{n}$;

2) следующая диаграмма коммутативна:

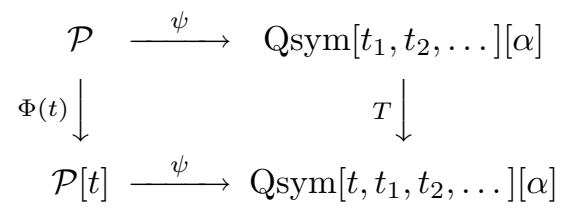

Тогда $\psi=\mathcal{F}_{\mathcal{P}}$. 
ДокАЗАтЕльСтво. Из условия 1$)$ следует, что $\psi(\alpha, 0,0, \ldots)(P)=\xi_{\alpha} P$ для любого элемента $P \in \mathcal{P}$.

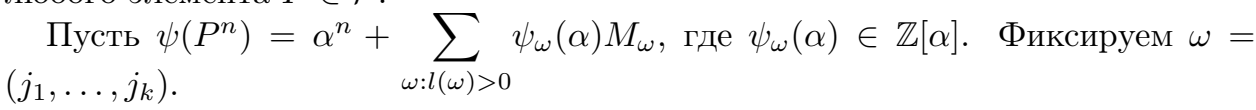

Так как $\psi\left(\alpha, t, t_{1}, t_{2}, \ldots\right)\left(P^{n}\right)=\psi\left(\alpha, t_{1}, t_{2}, \ldots\right)\left(\Phi(t) P^{n}\right)$, то

$$
\psi\left(\alpha, y_{1}, y_{2}, \ldots, y_{k}, t_{1}, t_{2}, \ldots\right)\left(P^{n}\right)=\psi\left(\alpha, t_{1}, t_{2}, \ldots\right)\left(\Phi\left(y_{k}\right) \cdots \Phi\left(y_{1}\right) P^{n}\right) .
$$

Поэтому

$$
\begin{aligned}
\psi\left(\alpha, y_{1}, \ldots, y_{k}, 0,0, \ldots\right) & =\psi(\alpha, 0,0, \ldots)\left(\Phi\left(y_{k}\right) \cdots \Phi\left(y_{1}\right) P^{n}\right) \\
& =\xi_{\alpha} \Phi\left(y_{k}\right) \cdots \Phi\left(y_{1}\right) P^{n}=\mathcal{F}_{\mathcal{P}}\left(\alpha, y_{1}, \ldots, y_{k}, 0,0, \ldots\right) .
\end{aligned}
$$

Следовательно, $\psi_{\omega}=f_{S(\omega)} \alpha^{n-|\omega|}$. Так как это рассуждение верно для всех композиций $\omega$, то $\psi=\mathcal{F}_{\mathcal{P}}$.

ЗАмечание 6.20. Отображение $T: \operatorname{Qsym}\left[t_{1}, t_{2}, \ldots\right][\alpha] \rightarrow \operatorname{Qsym}\left[t, t_{1}, \ldots\right][\alpha]-$ изоморфизм, в то время как отображение $\Phi(t): \mathcal{P} \rightarrow \mathcal{P}[t]$ - только мономорфизм, образ которого описывается условием $P(t) \in \Phi(t)(\mathcal{P})$ тогда и только тогда, когда $\Phi(-t) P(t) \in \mathcal{P}$.

Согласно утверждению 6.11, на кольце простых многогранников мы имеем:

$$
\mathcal{F}_{\mathcal{P}}\left(\alpha, t_{1}, t_{2}, \ldots\right)(P)=\mathcal{F}_{\mathcal{P}, 1}\left(\alpha, t_{1}+t_{2}+\cdots\right)(P)=\mathcal{F}_{\mathcal{P}, 1}\left(\alpha, \sigma_{1}\right)(P),
$$

поэтому образ кольца $\mathcal{P}_{s}$ принадлежит подкольцу $\mathbb{Z}\left[\alpha, \sigma_{1}\right]$. С другой стороны, $\left.\Phi(t)\right|_{\mathcal{P}_{\mathrm{s}}}=\left.e^{t d}\right|_{\mathcal{P}_{\mathrm{s}}}$, поэтому выполнено условие

$$
\mathcal{F}_{\mathcal{P}, 1}\left(\alpha, t_{1}\right)\left(e^{t d} P\right)=\mathcal{F}_{\mathcal{P}, 1}\left(\alpha, t+t_{1}\right)(P) .
$$

УтВеРЖДЕНИЕ 6.21. Пусть $\psi: \mathcal{P}_{\mathrm{s}} \rightarrow \mathbb{Z}[\alpha, t]-$ гомоморбизм абелевых групп такой, что

1) $\psi(\alpha, 0)\left(P^{n}\right)=\alpha^{n}$ для любого простого $n$-мерного многогранника $P^{n}$;

2) выполнено одно из следующих двух эквивалентных условий:

а) $\psi\left(\alpha, t_{1}\right)\left(e^{t d} P\right)=\psi\left(\alpha, t+t_{1}\right)(P)$ для любого элемента $P \in \mathcal{P}_{\mathrm{s}}$;

b) $\psi(\alpha, t)(d P)=\frac{\partial}{\partial t} \psi(\alpha, t)(P)$ для любого элемента $P \in \mathcal{P}_{\mathrm{s}}$.

Тогда $\psi=\mathcal{F}_{\mathcal{P}, 1}$.

ДоКАЗАТЕЛЬСтво. Пусть условия 1) и 2а) выполнены. Тогда для любого элемента $P \in \mathcal{P}_{\mathrm{s}}$ имеем:

$$
\begin{aligned}
\psi\left(\alpha, t+t_{1}\right)(P) & =\psi\left(\alpha, t_{1}\right)\left(e^{t d} P\right)=\psi(\alpha, 0)\left(e^{\left(t+t_{1}\right) d} P\right) \\
& =\xi_{\alpha} \Phi\left(t+t_{1}\right) P=\mathcal{F}_{\mathcal{P}, 1}\left(\alpha, t+t_{1}\right)(P) .
\end{aligned}
$$

Осталось доказать, что условия $2 \mathrm{a})$ и $2 \mathrm{~b})$ эквивалентны.

Если $\psi\left(\alpha, t_{1}\right)\left(e^{t d} P\right)=\psi\left(\alpha, t+t_{1}\right)(P)$, то $\psi(\alpha, t)(P)=\psi(\alpha, 0)\left(e^{t d} P\right)$, поэтому

$$
\begin{aligned}
\frac{\partial}{\partial t} \psi(\alpha, t)(P) & =\frac{\partial}{\partial t} \psi(\alpha, 0)\left(e^{t d} P\right)=\psi(\alpha, 0)\left(\frac{\partial}{\partial t} e^{t d} P\right) \\
& =\psi(\alpha, 0)\left(e^{t d} d P\right)=\psi(\alpha, t)(d P) .
\end{aligned}
$$

Пусть теперь $\psi(\alpha, t)(d P)=\frac{\partial}{\partial t} \psi(\alpha, t)(P)$ для любого элемента $P \in \mathcal{P}_{\mathrm{s}}$. Тогда $\frac{\partial}{\partial t} \psi\left(\alpha, t_{1}\right)\left(e^{t d} P\right)=\psi\left(\alpha, t_{1}\right)\left(\frac{\partial}{\partial t} e^{t d} P\right)=\psi\left(\alpha, t_{1}\right)\left(d\left(e^{t d} P\right)\right)=\frac{\partial}{\partial t_{1}} \psi\left(\alpha, t_{1}\right)\left(e^{t d} P\right)$. 
Поэтому $\left(\frac{\partial}{\partial t}-\frac{\partial}{\partial t_{1}}\right) \psi\left(\alpha, t_{1}\right)\left(e^{t d} P\right)=0$, следовательно, полином $\psi\left(\alpha, t_{1}\right)\left(e^{t d} P\right) \in$ $\mathbb{Z}\left[\alpha, t, t_{1}\right]$ можно представить как полином от переменных $\alpha$ и $t+t_{1}$.

Тогда $\psi\left(\alpha, t_{1}\right)\left(e^{t d} P\right)=\psi\left(\alpha, t+t_{1}\right)\left(e^{0 d} P\right)=\psi\left(\alpha, t+t_{1}\right)(P)$.

Таким образом, условия $2 \mathrm{a})$ и $2 \mathrm{~b})$ действительно эквивалентны.

ЗАмечание 6.22. Утверждение 6.21 в форме 1), 2b) было доказано в работе [2] и равносильно утверждению (ii) теоремы 1.55 .

6.4. Операторы бипирамиды и конуса. Введем аналоги операторов бипирамиды и конуса на кольцах $\mathcal{Q} \operatorname{sym}$ и $\mathcal{Q} \operatorname{sym}[\alpha]$.

УтвеРЖДЕНИЕ 6.23. (i) Введем операторы $C_{\mathcal{P}}, \mathscr{A}_{\mathcal{P}}: \mathcal{Q} \operatorname{sym}[\alpha] \rightarrow \mathcal{Q} \operatorname{sym}[\alpha]$ :

$$
\begin{aligned}
& C_{\mathcal{P}} g=\left(\alpha+\sigma_{1}\right) g+\sum_{n=1}^{\infty} t_{n} g\left(t_{n}, t_{1}, \ldots, t_{n-1}, 0,0, \ldots\right) ; \\
& \mathscr{A}_{\mathcal{P}} g=\alpha g(\alpha, 0,0, \ldots)+t_{1} g\left(\alpha, t_{1}, t_{2}, \ldots\right)+\sum_{n=2}^{\infty}\left(t_{n}+t_{n-1}\right) g\left(\alpha, t_{n}, t_{n+1}, \ldots\right) .
\end{aligned}
$$

Тогда $\mathcal{F}_{\mathcal{P}}(C P)=C_{\mathcal{P}} \mathcal{F}_{\mathcal{P}}(P)$ и $\mathcal{F}_{\mathcal{P}}(\mathscr{A} P)=\mathscr{A}_{\mathcal{P}} \mathcal{F}_{\mathcal{P}}(P)$ для всех $P \in \mathcal{P}$.

(ii) Введем операторы $C_{\mathcal{R} \mathcal{P}}, \mathscr{A}_{\mathcal{R} \mathcal{P}}: \mathcal{Q} \operatorname{sym}[\alpha] \rightarrow \mathcal{Q} \operatorname{sym}[\alpha]:$

$$
\begin{aligned}
C_{\mathcal{R P}} g=\left(\alpha+\sigma_{1}\right) g & \\
\mathscr{A}_{\mathcal{R} \mathcal{P}} g=\alpha & (g(\alpha, 0,0, \ldots)+g(0,0, \ldots))+t_{1} g\left(\alpha, t_{1}, t_{2}, \ldots\right) \\
& +\sum_{n=2}^{\infty}\left(t_{n}+t_{n-1}\right) g\left(\alpha, t_{n}, t_{n+1}, \ldots\right) .
\end{aligned}
$$

Тогда $\mathcal{F}_{\mathcal{R} \mathcal{P}}(C P)=C_{\mathcal{R} \mathcal{P}} \mathcal{F}_{\mathcal{R} \mathcal{P}}(P)$ u $\mathcal{F}_{\mathcal{R P}}(\mathscr{A} P)=\mathscr{A}_{\mathcal{R} \mathcal{P}} \mathcal{F}_{\mathcal{R} \mathcal{P}}(P)$ для всех $P \in \mathcal{R} \mathcal{P}$.

(iii) Введем операторы $C_{0}, \mathscr{A}_{0}: \mathcal{Q}$ sym $\rightarrow \mathcal{Q}$ sym:

$$
C_{0} g=\sigma_{1} g ; \quad \mathscr{A}_{0} g=t_{1} g\left(t_{1}, t_{2}, \ldots\right)+\sum_{n=2}^{\infty}\left(t_{n}+t_{n-1}\right) g\left(t_{n}, t_{n+1}, \ldots\right) .
$$

Тогда $\mathcal{F}_{\varepsilon}(C P)=C_{0} \mathcal{F}_{\varepsilon}(P)$ и $\mathcal{F}_{\varepsilon}(\mathscr{A} P)=\mathscr{A}_{0} \mathcal{F}_{\varepsilon}(P)$ для всех $P \in \mathcal{R} \mathcal{P}$.

ДокАЗАТЕЛьство. Пользуясь критерием (33), легко показать, что для любого элемента $g \in \mathcal{Q} \operatorname{sym}[\alpha]$ выражения $C_{\mathcal{P}} g, C_{\mathcal{R} \mathcal{P}} g, \mathscr{A}_{\mathcal{P}} g$ и $\mathscr{A}_{\mathcal{R} P} g$ тоже принадлежат кольцу $\mathcal{Q} s y m[\alpha]$, а для любого элемента $g \in \mathcal{Q} s y m$ выражения $C_{0} g$ и $\mathscr{A}_{0} g$ тоже принадлежат $\mathcal{Q} s y m$. Далее, на кольце $\mathcal{P}$ имеем:

$$
\begin{aligned}
& \Phi_{1} C=\Phi\left(t_{1}\right) C=\left(C+t_{1}\right) \Phi\left(t_{1}\right)+t_{1} \xi_{t_{1}}, \\
& \Phi_{2} C=\Phi\left(t_{2}\right) \Phi\left(t_{1}\right) C=\left(C+t_{2}+t_{1}\right) \Phi\left(t_{2}\right) \Phi\left(t_{1}\right)+t_{2} \xi_{t_{2}} \Phi\left(t_{1}\right)+t_{1} \xi_{t_{1}}, \\
& \Phi_{n} C=\left(C+t_{n}+\cdots+t_{1}\right) \Phi\left(t_{n}\right) \Phi\left(t_{n-1}\right) \cdots \Phi\left(t_{1}\right) \\
& +t_{n} \xi_{t_{n}} \Phi\left(t_{n-1}\right) \cdots \Phi\left(t_{1}\right)+\cdots+t_{1} \xi_{t_{1}} \\
& \Phi_{\infty} C=\left(C+\sigma_{1}\right) \Phi_{\infty}+\sum_{n=1}^{\infty} t_{n} \mathcal{F}_{\mathcal{P}}\left(t_{n}, t_{1}, \ldots, t_{n-1}, 0,0, \ldots\right) .
\end{aligned}
$$

Используя то, что $\xi_{\alpha} C=\alpha \xi_{\alpha}$, получаем:

$$
\begin{aligned}
\mathcal{F}_{\mathcal{P}}(C P)=\xi_{\alpha} & \Phi_{\infty} C P=\left(\alpha+\sigma_{1}\right) \mathcal{F}_{\mathcal{P}}\left(\alpha, t_{1}, t_{2}, \ldots\right) \\
& +\sum_{n=1}^{\infty} t_{n} \mathcal{F}_{\mathcal{P}}\left(t_{n}, t_{1}, \ldots, t_{n-1}, 0,0, \ldots\right)=C_{\mathcal{P}} \mathcal{F}_{\mathcal{P}}(P) .
\end{aligned}
$$


Оператор конуса на кольце $\mathcal{R} \mathcal{P}$ задается умножением на элемент $x=\mathrm{pt}$, а гомоморфизмы $\mathcal{F}_{\mathcal{R} \mathcal{P}}$ и $\mathcal{F}_{\varepsilon}$ являются кольцевыми, поэтому

$$
\begin{gathered}
\mathcal{F}_{\mathcal{R} \mathcal{P}}(C P)=\mathcal{F}_{\mathcal{R P}}(x P)=\mathcal{F}_{\mathcal{R P}}(x) \mathcal{F}_{\mathcal{R P}}(P)=\left(\alpha+\sigma_{1}\right) \mathcal{F}_{\mathcal{R} \mathcal{P}}(P)=C_{\mathcal{R} \mathcal{P}} \mathcal{F}_{\mathcal{R P}}(P), \\
\mathcal{F}_{\varepsilon}(C P)=\mathcal{F}_{\varepsilon}(x P)=\mathcal{F}_{\varepsilon}(x) \mathcal{F}_{\varepsilon}(P)=\sigma_{1} \mathcal{F}_{\varepsilon}(P)=C_{0} \mathcal{F}_{\varepsilon}(P) .
\end{gathered}
$$

На кольце $\mathrm{R}$ имеем:

$$
\begin{gathered}
\Phi_{1} \mathscr{A}=\Phi\left(t_{1}\right) \mathscr{A}=\mathscr{A}+t_{1}+t_{1} \Phi\left(t_{1}\right) \\
\Phi_{2} \mathscr{A}=\Phi\left(t_{2}\right) \Phi\left(t_{1}\right) \mathscr{A}=\mathscr{A}+t_{2}+\left(t_{2}+t_{1}\right) \Phi\left(t_{2}\right)+t_{1} \Phi\left(t_{2}\right) \Phi\left(t_{1}\right), \\
\ldots \ldots \ldots \ldots \ldots \ldots \ldots \ldots \ldots \ldots \ldots \ldots \ldots \ldots \ldots \ldots \ldots \ldots \ldots \ldots \\
\Phi_{n} \mathscr{A}=\mathscr{A}+t_{n}+\left(t_{n}+t_{n-1}\right) \Phi\left(t_{n}\right) \\
\quad+\left(t_{n-1}+t_{n-2}\right) \Phi\left(t_{n}\right) \Phi\left(t_{n-1}\right)+\cdots+t_{1} \Phi\left(t_{n}\right) \cdots \Phi\left(t_{1}\right), \\
\Phi_{\infty} \mathscr{A}=\mathscr{A}+t_{1} \Phi_{\infty}\left(t_{1}, t_{2}, \ldots\right)+\sum_{n=2}^{\infty}\left(t_{n}+t_{n-1}\right) \Phi_{\infty}\left(t_{n}, t_{n+1}, \ldots\right) .
\end{gathered}
$$

Используя то, что $\xi_{\alpha} \mathscr{A}=\alpha \xi_{\alpha}$ на $\mathcal{P}$ и $\varepsilon_{\alpha}(\mathscr{A} P)=\alpha\left(\varepsilon_{\alpha}+\varepsilon_{0}\right)$ на кольце $\mathcal{R} \mathcal{P}$, получаем

$$
\begin{aligned}
\mathcal{F}_{\mathcal{P}}(\mathscr{A} P) & =\xi_{\alpha} \Phi_{\infty} \mathscr{A} P=\mathscr{A}_{\mathcal{P}} \mathcal{F}_{\mathcal{P}}(P), \\
\mathcal{F}_{\mathcal{R} \mathcal{P}}(\mathscr{A} P) & =\varepsilon_{\alpha} \Phi_{\infty} \mathscr{A} P=\mathscr{A}_{\mathcal{R} \mathcal{P}} \mathcal{F}_{\mathcal{R P}}(P), \\
\mathcal{F}_{\varepsilon}(\mathscr{A} P) & =\varepsilon_{0} \Phi_{\infty} \mathscr{A} P=\mathscr{A}_{0} \mathcal{F}_{\varepsilon}(P) .
\end{aligned}
$$

Утверждение доказано.

Определим отображения $B_{\mathcal{P}}, B_{\mathcal{R P}}: \mathcal{Q} \operatorname{sym}[\alpha] \rightarrow \mathcal{Q} s y m[\alpha]$ и $B_{0}: \mathcal{Q} s y m \rightarrow$ $\mathcal{Q}$ sym как $B_{\mathcal{P}}=2 C_{\mathcal{P}}-\mathscr{A}_{\mathcal{P}}, B_{\mathcal{R P}}=2 C_{\mathcal{R} \mathcal{P}}-\mathscr{A}_{\mathcal{R} \mathcal{P}}, B_{0}=2 C_{0}-\mathscr{A}_{0}$. Тогда

$$
\mathcal{F}_{\mathcal{P}}(B P)=B_{\mathcal{P}} \mathcal{F}_{\mathcal{P}}(P), \quad \mathcal{F}_{\mathcal{R} \mathcal{P}}(B P)=B_{\mathcal{R} \mathcal{P}} \mathcal{F}_{\mathcal{R} \mathcal{P}}(P), \quad \mathcal{F}_{\varepsilon}(B P)=B_{0} \mathcal{F}_{\varepsilon}(P) .
$$

УтВеРЖДЕНИЕ 6.24. Введем отображения $\mathscr{D}_{\mathcal{P}}, \mathscr{D}_{\mathcal{R} \mathcal{P}}: \mathcal{Q} \operatorname{sym}[\alpha] \rightarrow \mathcal{Q} \operatorname{sym}[\alpha]$ :

$$
\mathscr{D}_{\mathcal{P}} g=\alpha \sigma_{1} g(\alpha, 0,0, \ldots)+\sum_{n=2}^{\infty}\left(t_{1}+\cdots+t_{n-1}\right)\left(t_{n-1}+t_{n}\right) g\left(\alpha, t_{n}, t_{n+1}, \ldots\right) \text {; }
$$

$$
\mathscr{D}_{\mathcal{R} \mathcal{P}} g=\alpha\left(\alpha+\sigma_{1}\right) g(0,0, \ldots)+\mathscr{D}_{\mathcal{P}} g
$$

$u \mathscr{D}_{0}: \mathcal{Q}$ sym $\rightarrow \mathcal{Q}$ sym $:$

$$
\mathscr{D}_{\mathcal{P}} g=\sum_{n=2}^{\infty}\left(t_{1}+\cdots+t_{n-1}\right)\left(t_{n-1}+t_{n}\right) g\left(t_{n}, t_{n+1}, \ldots\right) .
$$

Тогда $\mathcal{F}_{\mathcal{P}}(\mathscr{D} P)=\mathscr{D}_{\mathcal{P}} \mathcal{F}_{\mathcal{P}}(P)$ для всех $P \in \mathcal{P} u \mathcal{F}_{\mathcal{R} \mathcal{P}}(\mathscr{D} P)=\mathscr{D}_{\mathcal{R} \mathcal{P}} \mathcal{F}_{\mathcal{R P}}(P)$, $\mathcal{F}_{\varepsilon}(\mathscr{D} P)=\mathscr{D}_{0} \mathcal{F}_{\varepsilon}(P)$ для всех $P \in \mathcal{R} \mathcal{P}$.

ДокАзАтЕльство. Пользуясь критерием (35), легко показать, что для полинома $g \in \mathcal{Q} \operatorname{sym}[\alpha]$ выражения $\mathscr{D}_{\mathcal{P}} g$ и $\mathscr{D}_{\mathcal{R} \mathcal{P}} g$ также принадлежит $\mathcal{Q} \operatorname{sym}[\alpha]$, а для $g \in \mathcal{Q} s y m$ выражение $\mathscr{D}_{0} g$ также принадлежит $\mathcal{Q} s y m$. На кольце $\mathrm{R}$ имеем:

$$
\begin{aligned}
& \Phi_{1} \mathscr{D}=\Phi\left(t_{1}\right) \mathscr{D}=\mathscr{D}+\left(\mathscr{A}+t_{1}\right) t_{1} \\
& \Phi_{2} \mathscr{D}=\Phi\left(t_{2}\right) \Phi\left(t_{1}\right) \mathscr{D}=\mathscr{D}+\left(\mathscr{A}+t_{2}\right)\left(t_{1}+t_{2}\right)+t_{1}\left(t_{1}+t_{2}\right) \Phi\left(t_{2}\right) ; \\
& \Phi_{n} \mathscr{D}=\mathscr{D}+\left(\mathscr{A}+t_{n}\right)\left(t_{1}+\cdots+t_{n}\right)+\left(t_{1}+\cdots+t_{n-1}\right)\left(t_{n-1}+t_{n}\right) \Phi\left(t_{n}\right) \\
& +\left(t_{1}+\cdots+t_{n-2}\right)\left(t_{n-2}+t_{n-1}\right) \Phi\left(t_{n}\right) \Phi\left(t_{n-1}\right)+\cdots+t_{1}\left(t_{1}+t_{2}\right) \Phi\left(t_{n}\right) \cdots \Phi\left(t_{2}\right) \text {; } \\
& \Phi_{\infty} \mathscr{D}=\mathscr{D}+\mathscr{A}\left(t_{1}+t_{2}+\cdots\right)+\sum_{n=2}^{\infty}\left(t_{1}+\cdots+t_{n-1}\right)\left(t_{n-1}+t_{n}\right) \Phi_{\infty}\left(t_{n}, t_{n+1}, \ldots\right) .
\end{aligned}
$$


Так как $\xi_{\alpha} \mathscr{D}=0$ на кольце $\mathcal{P}$ и $\varepsilon_{\alpha} \mathscr{D}=\alpha^{2} \varepsilon_{0}$ на кольце $\mathcal{R} \mathcal{P}$ (в частности, $\left.\varepsilon_{0} \mathscr{D}=0\right)$, то получаем:

$$
\begin{aligned}
\mathcal{F}_{\mathcal{P}}(\mathscr{D} P)=\xi_{\alpha} & \Phi_{\infty}(\mathscr{D} P)=\alpha \sigma_{1} \mathcal{F}_{\mathcal{P}}(\alpha, 0,0, \ldots) \\
& +\sum_{n=2}^{\infty}\left(t_{1}+\cdots+t_{n-1}\right)\left(t_{n-1}+t_{n}\right) \mathcal{F}_{\mathcal{P}}\left(\alpha, t_{n}, t_{n+1}, \ldots\right)=\mathscr{D}_{\mathcal{P}} \mathcal{F}_{\mathcal{P}}(P)
\end{aligned}
$$

и $\mathcal{F}_{\mathcal{R P}}(\mathscr{D} P)=\mathscr{D}_{\mathcal{R} \mathcal{P}} \mathcal{F}_{\mathcal{R} \mathcal{P}}(P), \mathcal{F}_{\varepsilon}(\mathscr{D} P)=\mathscr{D}_{0} \mathcal{F}_{\varepsilon}(P)$.

ПримеР 6.25. Имеем:

$$
\begin{gathered}
\mathscr{A}_{\mathcal{P}}\left[\alpha^{j_{1}}\right]=\alpha^{j_{1}}\left(\alpha+2 M_{(1)}\right) ; \\
\mathscr{A}_{\mathcal{P}}\left[\alpha^{j_{1}} M_{\left(j_{k}, \ldots, j_{2}\right)}\right]=\alpha^{j_{1}}\left(M_{\left(j_{k}+1, j_{k-1}, \ldots, j_{2}\right)}+2 M_{\left(1, j_{k}, \ldots, j_{2}\right)}\right) ; \\
\mathscr{D}_{\mathcal{P}}\left[\alpha^{j_{1}}\right]=\alpha^{j_{1}}\left(\alpha M_{(1)}+M_{(2)}+2 M_{(1,1)}\right)=\alpha^{j_{1}} M_{(1)}\left(\alpha+2 M_{(1)}\right) ; \\
\mathscr{D}_{\mathcal{P}}\left[\alpha^{j_{1}} M_{\left(j_{k}, \ldots, j_{2}\right)}\right]=\alpha^{j_{1}}\left(M_{\left(1, j_{k}+1, j_{k-1}, \ldots, j_{2}\right)}+M_{\left(2, j_{k}, \ldots, j_{2}\right)}+2 M_{\left(1,1, j_{k}, \ldots, j_{2}\right)}\right) .
\end{gathered}
$$

ОПРЕДЕлЕниЕ 6.26. Определим на кольце $\mathcal{Q} s y m[\alpha]$ правое действие алгебры $\mathcal{Q}=\mathbb{Z}\langle\boldsymbol{c}, \boldsymbol{d}\rangle$ условием $g \boldsymbol{c}=\mathscr{A}_{\mathcal{P}} g$ и $g \boldsymbol{d}=\mathscr{D}_{\mathcal{P}} g$.

Из следствия 1.89 и утверждений $6.23,6.24$ вытекает следующий результат.

УТВЕРЖДЕНИЕ 6.27. Отображение $\mathcal{F}_{\mathcal{P}}$ эквивариантно и $\mathcal{F}_{\mathcal{P}}(\mathcal{P})=1 \mathbb{Z}\langle\boldsymbol{c}, \boldsymbol{d}\rangle$.

\section{7. Структура кольца $\mathcal{D}^{*}$}

\section{1. Основная теорема.}

ОПРЕДЕЛЕниЕ 7.1. Обозначим через $\mathcal{D}^{*}$ градуированно двойственную к $\mathcal{D}$ алгебру Хопфа.

Отождествим алгебры Хопфа $\mathcal{Z}^{*}$ и $\mathcal{Q}$ sym. Имеют место эпиморфизмы $\mathscr{L}$ : $\mathcal{Z} \rightarrow \mathcal{D}$ и $\mathscr{R}: \mathcal{Z}^{\text {ор сор }} \rightarrow \mathcal{D}$, переводящие $Z_{i}$ в $d_{i}$. Поэтому кольцевые гомоморфизмы $\mathscr{L}^{*}$ и $\mathscr{R}^{*}=\varrho^{*} \mathscr{L}^{*}$ определяют два вложения кольца $\mathcal{D}^{*}$ в кольцо $\mathcal{Q} s y m$, связанные инволюцией $\varrho^{*}=*$. Из замечания 2.28 и теоремы 5.1 следует, что образ $\mathscr{L}\left(\mathcal{D}^{*}\right)$ инвариантен относительно инволюции $*$, поэтому $\mathscr{L}^{*}\left(\mathcal{D}^{*}\right)=\mathscr{R}^{*}\left(\mathcal{D}^{*}\right)$.

Вложение $\mathscr{L}^{*}$ является вложением алгебр Хопфа, в то время как для вложения $\mathscr{R}^{*}$ мы имеем: $\Delta \mathscr{R}^{*}=\tau_{\mathcal{Q} \text { sym, } \mathcal{Q} \text { sym }}\left(\mathscr{R}^{*} \otimes \mathscr{R}^{*}\right) \Delta$.

ОПРЕДЕЛЕНИЕ 7.2. Для любого $r \geqslant 0$ зададим гомоморфизм $\mathscr{R}_{r}^{*}: \mathcal{D}^{*} \rightarrow$ Qsym $\left[t_{1}, \ldots, t_{r}\right]$ формулой

$$
\mathscr{R}_{r}^{*}(\psi)=\mathscr{R}^{*}(\psi)\left(t_{1}, \ldots, t_{r}, 0,0, \ldots\right) .
$$

Так как ограничение $\operatorname{Qsym}\left[t_{1}, t_{2}, \ldots\right] \rightarrow \operatorname{Qsym}\left[t_{1}, \ldots, t_{r}\right]: g\left(t_{1}, \ldots, t_{r}, t_{r+1}\right.$, $\left.t_{r+2}, \ldots\right) \rightarrow g\left(t_{1}, \ldots, t_{r}, 0,0, \ldots\right)$ изоморфно на группах $\mathcal{Q}_{s y m}{ }^{2 n}, \quad n \leqslant r$, то отображение $\mathscr{R}_{r}^{*}$ изоморфно на группах $\mathcal{D}_{-2 n}^{*}, n \leqslant r$. Получим теперь основной результат этого раздела.

Tеорема 7.3. Подгруnпа $\mathscr{R}_{r}^{*}\left(\mathcal{D}_{-2 n}^{*}\right) \subset \mathrm{Qsym}\left[t_{1}, \ldots, t_{r}\right], r \geqslant n \geqslant 1$, cocmoum из однородных элементов степени $2 n$, удовлетворяющих уравнениям (58). 
ДоКАЗАТЕЛЬСТво. Кольцо $\mathscr{R}^{*}\left(\mathcal{D}^{*}\right) \subset \mathcal{Z}^{*}$ состоит из линейных функций $\psi \in \mathcal{Z}^{*}$, удовлетворяющих условию

$$
\left\langle\psi, z_{1} \Phi(t) \Phi(-t) z_{2}\right\rangle=\left\langle\psi, z_{1} z_{2}\right\rangle
$$

для всех $z_{1}, z_{2} \in \mathcal{Z}$, т. е. все коэффициенты при мономах $t^{k}, k \geqslant 1$, в левой части равенства равны нулю.

ЛЕмма 7.4. Для $r \geqslant 0$ мы имеем:

$$
\mathscr{R}_{r}^{*}(\psi)=\left\langle\psi, \Phi\left(t_{r}\right) \Phi\left(t_{r-1}\right) \cdots \Phi\left(t_{2}\right) \Phi\left(t_{1}\right)\right\rangle,
$$

где для $r=0$ произведение $\Phi\left(t_{r}\right) \cdots \Phi\left(t_{1}\right)$ равно единице.

ДоказАтельство. Так как $\mathscr{R}^{*}(\psi)=\sum_{\omega}\left\langle\mathscr{R}^{*}(\psi), Z_{\omega}\right\rangle=\sum_{\omega}\left\langle\psi, \mathscr{R} Z_{\omega}\right\rangle M_{\omega}$, то

$$
\begin{aligned}
\mathscr{R}_{r}^{*}(\psi) & =\sum_{\omega: l(\omega) \leqslant r}\left\langle\psi, \mathscr{R} Z_{\omega}\right\rangle M_{\omega}\left(t_{1}, \ldots, t_{r}\right) \\
& =\sum_{k=0}^{r} \sum_{\left(j_{1}, \ldots, j_{k}\right)}\left\langle\psi, d_{j_{k}} \cdots d_{j_{1}}\right\rangle M_{\left(j_{1}, \ldots, j_{k}\right)}\left(t_{1}, \ldots, t_{r}\right) \\
& =\sum_{k=0}^{r} \sum_{\left(j_{1}, \ldots, j_{k}\right)}\left\langle\psi, d_{j_{k}} \cdots d_{j_{1}}\right\rangle \sum_{1 \leqslant l_{1}<\cdots<l_{k} \leqslant r} t_{l_{1}}^{j_{1}} \cdots t_{l_{k}}^{j_{k}} \\
& =\sum_{k=0}^{r} \sum_{\left(j_{1}, \ldots, j_{k}\right)} \sum_{1 \leqslant l_{1}<\cdots<l_{k} \leqslant r}\left\langle\psi, d_{j_{k}} t_{l_{k}}^{j_{k}} \cdots d_{j_{1}} t_{l_{1}}^{j_{1}}\right\rangle=\left\langle\psi, \Phi\left(t_{r}\right) \cdots \Phi\left(t_{1}\right)\right\rangle,
\end{aligned}
$$

что и доказывает лемму.

Таким образом,

$$
\begin{gathered}
\mathscr{R}_{r}^{*}(\psi)\left(t_{1}, \ldots, t_{i},-t_{i}, \ldots, t_{r}\right)=\left\langle\psi, \Phi\left(t_{r}\right) \cdots \Phi\left(-t_{i}\right) \Phi\left(t_{i}\right) \cdots \Phi\left(t_{1}\right)\right\rangle \\
=\left\langle\psi, \Phi\left(t_{r}\right) \cdots \Phi(0) \Phi(0) \cdots \Phi\left(t_{1}\right)\right\rangle=\mathscr{R}_{r}^{*}(\psi)\left(t_{1}, \ldots, 0,0, \ldots, t_{r}\right) .
\end{gathered}
$$

$\mathrm{C}$ другой стороны, пусть $g \in \mathrm{Qsym}\left[t_{1}, \ldots, t_{r}\right]$ - однородный полином степени $2 n$, удовлетворяющий уравнениям (58). Рассмотрим единственную однородную функцию $\widetilde{g} \in \mathcal{Q} s y m$ такую, что $\operatorname{deg} \widetilde{g}=2 n$ и $\widetilde{g}\left(t_{1}, \ldots, t_{r}, 0,0, \ldots\right)=g$. Покажем, что $\widetilde{g} \in \mathscr{R}^{*}\left(\mathcal{D}^{*}\right)$.

Достаточно доказать соотношение (62) в случае, когда $z_{1}$ и $z_{2}$ - мономы. Так как $\Phi(t) \Phi(-t)=1+\left(2 Z_{2}-Z_{1}^{2}\right) t^{2}+\cdots$ и $\operatorname{deg} \widetilde{g}=2 n$, то случаи, когда $\operatorname{deg} z_{1}+\operatorname{deg} z_{2}$ равно $2 n$ и $2(n-1)$, тривиальны. Пусть $k \geqslant 2$ и

$$
z_{1}=Z_{\omega}, \quad z_{2}=Z_{\omega^{\prime}}, \quad \omega=\left(j_{1}, \ldots, j_{l}\right), \quad \omega^{\prime}=\left(j_{1}^{\prime}, \ldots, j_{l^{\prime}}^{\prime}\right), \quad|\omega|+\left|\omega^{\prime}\right|=n-k .
$$

Тогда нам нужно доказать единственное равенство:

$$
\left\langle\widetilde{g}, Z_{\omega}\left(\sum_{i=0}^{k}(-1)^{i} Z_{k-i} Z_{i}\right) Z_{\omega^{\prime}}\right\rangle=0 .
$$

Рассмотрим уравнение

$$
\begin{array}{r}
g\left(t_{1}, \ldots, t_{l}, t_{l+1},-t_{l+1}, t_{l+3}, \ldots, t_{l+2+l^{\prime}}, \ldots, t_{r}\right) \\
\quad=g\left(t_{1}, \ldots, t_{l}, 0,0, t_{l+3}, \ldots, t_{l+2+l^{\prime}}, \ldots, t_{r}\right) .
\end{array}
$$


Слева коэффициент при мономе $t_{1}^{j_{1}} \cdots t_{l}^{j_{l}} t_{l+1}^{k} t_{l+3}^{j_{1}^{\prime}} \cdots t_{l+2+l^{\prime}}^{j_{\prime^{\prime}}^{\prime}}$ равен в точности

$$
\sum_{i=0}^{k}(-1)^{i}\left\langle\widetilde{g}, Z_{\omega} Z_{k-i} Z_{i} Z_{\omega^{\prime}}\right\rangle=\left\langle\widetilde{g}, Z_{\omega}\left(\sum_{i=0}^{k}(-1)^{i} Z_{k-i} Z_{i}\right) Z_{\omega^{\prime}}\right\rangle,
$$

в то время как справа он равен нулю, поэтому $\left\langle\widetilde{g}, Z_{\omega} \Phi(t) \Phi(-t) Z_{\omega^{\prime}}\right\rangle=0$.

Таким образом, соотношения (62) для функции $\widetilde{g} \in \mathcal{Q} s y m$ выполнены, поэтому $\widetilde{g}=\mathscr{R}^{*} \psi, \psi \in \mathcal{D}^{*}$. Следовательно, $g=\widetilde{g}\left(t_{1}, \ldots, t_{r}, 0,0, \ldots\right)=\mathscr{R}_{r}^{*}(\psi)$.

СлеДСТвиЕ 7.5. Образ гомоморфизма $\mathcal{F}_{\varepsilon}: \mathcal{R P} \rightarrow \mathcal{Q}$ sym совпадает с кольиом $\mathscr{L}^{*}\left(\mathcal{D}^{*}\right)=\mathscr{R}^{*}\left(\mathcal{D}^{*}\right)$.

ОПРЕДЕЛЕниЕ 7.6. На выражениях вида (32) ограниченной степени зададим операции $\Theta_{k}$ формулами

$$
\Theta_{k} g\left(t_{1}, t_{2}, \ldots\right)=g\left(t_{1}, \ldots, t_{k-1}, t,-t, t_{k}, t_{k+1}, \ldots\right) .
$$

СлЕДСТвиЕ 7.7. Образ $\mathscr{R}^{*}\left(\mathcal{D}^{*}\right) \subset \mathcal{Q}$ sym определяется условием

$g \in \mathscr{R}^{*}\left(\mathcal{D}^{*}\right)$ тогда и толъко тогда, когда $\Theta_{k} g=g$ для всех $k \geqslant 1$.

ДокАЗАтельство. Пусть $g \in \mathcal{Q} \operatorname{sym}^{2 n}$, причем $-g=\mathscr{R}^{*}(\psi), \psi \in \mathcal{D}^{*}$. Каждый моном выражения $g\left(t_{1}, \ldots, t_{k-1}, t,-t, t_{k}, t_{k+1}, \ldots\right)$ входит с тем же самым коэффициентом в полином

$$
g_{r+2}\left(t_{1}, \ldots, t_{k-1}, t,-t, t_{k}, \ldots, t_{r}\right)=g\left(t_{1}, \ldots, t_{k-1}, t,-t, t_{k}, \ldots, t_{r}, 0,0, \ldots\right)
$$

для достаточно большого $r$. По теореме 7.3

$$
g_{r+2}\left(t_{1}, \ldots, t_{k-1}, t,-t, t_{k}, \ldots, t_{r}\right)=g_{r+2}\left(t_{1}, \ldots, t_{k-1}, 0,0, t_{k}, \ldots, t_{r}\right),
$$

следовательно, все мономы, содержащие переменную $t$, имеют нулевой коэффициент. Поэтому и все мономы в выражении $g\left(t_{1}, \ldots, t_{k-1}, t,-t, t_{k}, \ldots\right)$, содержащие $t$, тоже имеют нулевой коэффициент. Следовательно,

$$
g\left(t_{1}, \ldots, t_{k-1}, t,-t, t_{k}, \ldots\right)=g\left(t_{1}, \ldots, t_{k-1}, 0,0, t_{k}, \ldots\right)=g\left(t_{1}, \ldots, t_{k-1}, t_{k}, \ldots\right) .
$$

С другой стороны, пусть $g \in \mathcal{Q} s m^{2 n}$ - квазисимметрическая функция такая, что $\Theta_{k} g=g$ для всех $k \geqslant 1$. Тогда для квазисимметрической функции

$$
g_{n}\left(t_{1}, \ldots, t_{n}\right)=g\left(t_{1}, \ldots, t_{n}, 0,0, \ldots\right) \in \operatorname{Qsym}\left[t_{1}, \ldots, t_{n}\right]
$$

мы имеем:

$$
\begin{gathered}
g_{n}\left(t_{1}, \ldots, t_{i-1}, t_{i},-t_{i}, t_{i+2}, \ldots, t_{n}\right)=g\left(t_{1}, \ldots, t_{i-1}, t_{i},-t_{i}, t_{i+2}, \ldots, t_{n}, 0,0, \ldots\right) \\
\quad=g\left(t_{1}, \ldots, t_{i-1}, t_{i+2}, \ldots, t_{n}, 0,0, \ldots\right)=g_{n}\left(t_{1}, \ldots, t_{i-1}, t_{i+2}, \ldots, t_{n}, 0,0\right) \\
\quad=g_{n}\left(t_{1}, \ldots, t_{i-1}, 0,0, t_{i+2}, \ldots, t_{n}\right)
\end{gathered}
$$

для $1 \leqslant i \leqslant n-1$. По теореме 7.3 мы получаем, что $g_{n}=\mathscr{R}_{n}^{*}(\psi), \psi \in \mathcal{D}_{-2 n}^{*}$. Так как ограничение $g\left(t_{1}, t_{2}, \ldots\right) \rightarrow g\left(t_{1}, \ldots, t_{n}, 0,0, \ldots\right)$ изоморфно на группе $\mathcal{Q} \operatorname{sym}^{2 n}$, то $g=\mathscr{R}^{*} \psi$.

ЗАмЕчАниЕ 7.8. Мы можем аналогично определить отображение $\mathscr{L}_{r}^{*}: \mathcal{D}^{*} \rightarrow$ Qsym $\left[t_{1}, \ldots, t_{r}\right]$ :

$$
\mathscr{L}_{r}^{*}(\psi)\left(t_{1}, \ldots, t_{r}\right)=\mathscr{L}^{*}(\psi)\left(t_{1}, \ldots, t_{r}, 0,0, \ldots\right) .
$$

Тогда $\mathscr{R}_{r}^{*}=\varrho^{*} \mathscr{L}_{r}^{*}, \varrho^{*}=*$. Кроме того, образ $\mathscr{L}_{r}^{*}$ инвариантен относительно инволюции $*$, поэтому $\mathscr{R}^{*}\left(\mathcal{D}^{*}\right)=\mathscr{L}^{*}\left(\mathcal{D}^{*}\right)$. Следовательно, теорема 7.3 и следствие 7.7 верны и для отображений $\mathscr{L}_{r}^{*}$ и $\mathscr{L}$. 
УтВеРЖДЕНИЕ 7.9. Имеем $\mathcal{D}^{*} \otimes \mathbb{Q} \simeq \mathbb{Q}\left[\mathrm{LYN}_{\text {odd }}\right] u \operatorname{dim} \mathcal{D}_{-2 n}^{*} \otimes \mathbb{Q}=c_{n-1}$.

ДокАЗАТЕЛЬство. Это следует из утверждения 5.12, следствия 3.6 теоремы о структуре тасовочной алгебры и следствия 5.8 .

7.2. Приложения. Пусть $\mathcal{D}^{*}[\alpha]$ - градуированное кольцо полиномов от переменной $\alpha$ степени -2 , где для элемента $\psi_{i}$ градуировки $2 i$ элемент $\psi_{i} \alpha^{j}$ имеет градуировку $-2(i+j)$.

УтвеРЖДЕНИЕ 7.10. Любой кольцевой гомоморфизм $\varkappa: \mathrm{R} \rightarrow \mathcal{A}$, где $\mathcal{A}-$ ассоииативное кольцо с умножением $\mu_{\mathcal{A}}: \mathcal{A} \otimes \mathcal{A} \rightarrow \mathcal{A}$ и единицей $\imath_{\mathcal{A}}: \mathbb{Z} \rightarrow \mathcal{A}$, индуцирует гомоморфизм колеи, $\varphi_{\varkappa}: \mathrm{R} \rightarrow \mathcal{D}^{*} \otimes \mathcal{A}$,

$$
\left\langle\varphi_{\varkappa}(P), D\right\rangle=\varkappa(D P),
$$

где $\mathcal{D}^{*} \otimes \mathcal{A} \simeq \sum_{n \geqslant 0} \operatorname{Hom}\left(\mathcal{D}_{-2 n}, A\right)-$ кольцо с операцией свертки $\psi_{1} \star \psi_{2}(D)=$ $\mu_{\mathcal{A}}\left(\psi_{1} \otimes \psi_{2}\right) \Delta(D)$ и единищей $\varepsilon_{\mathcal{A}}(D)=\imath_{A} \varepsilon(D)$.

ДокАЗАТЕЛЬСтво. Действительно,

$$
\begin{aligned}
\left\langle\varphi_{\varkappa}(P Q), D\right\rangle & =\varkappa(D(P Q))=\varkappa \mu \Delta D(P \otimes Q)=\mu_{\mathcal{A}}(\varkappa \otimes \varkappa) \Delta D(P \otimes Q) \\
& =\mu_{\mathcal{A}}\left\langle\varphi_{\varkappa}(P) \otimes \varphi_{\varkappa}(Q), \Delta D\right\rangle=\left\langle\varphi_{\varkappa}(P) \star \varphi_{\varkappa}(Q), D\right\rangle, \\
\left\langle\varphi_{\varkappa}(1), D\right\rangle & =\varkappa(D 1)=\varkappa(\varepsilon(D) \cdot 1)=\iota_{\mathcal{A}} \varepsilon(D)=\left\langle\varepsilon_{\mathcal{A}}, D\right\rangle .
\end{aligned}
$$

УТВЕРЖДЕНИЕ 7.11. Мы имеем $\left(\mathscr{R}^{*} \otimes 1\right) \varphi_{\varkappa}=\mathcal{F}_{\varkappa}$.

ДокАЗАТЕльство. В самом деле,

$$
\begin{aligned}
\left(\mathscr{R}^{*} \otimes 1\right) \varphi_{\varkappa}(P) & =\sum_{\omega} M_{\omega} \otimes\left\langle\mathscr{R}^{*} \varphi_{\varkappa}(P), Z_{\omega}\right\rangle=\sum_{\omega} M_{\omega} \otimes\left\langle\varphi_{\varkappa}(P), \mathscr{R} Z_{\omega}\right\rangle \\
& =\sum_{\omega} M_{\omega^{*}} \otimes\left\langle\varphi_{\varkappa}(P), D_{\omega}\right\rangle=\sum_{\omega} M_{\omega^{*}} \otimes \varkappa\left(D_{\omega} P\right)=\mathcal{F}_{\varkappa}(P) .
\end{aligned}
$$

ПримеР 7.12. Описанная конструкция дает следующие важные гомоморфизмы.

1) Положим $\varphi_{\mathcal{P}, \alpha}=\varphi_{\xi_{\alpha}}: \mathcal{P} \rightarrow \mathcal{D}^{*}[\alpha]$. Тогда $\mathscr{R}^{*} \varphi_{\mathcal{P}, \alpha}=\mathcal{F}_{\mathcal{P}}$.

2) Положим $\varphi_{\mathcal{R} \mathcal{P}, \alpha}=\varphi_{\varepsilon_{\alpha}}: \mathcal{R P} \rightarrow \mathcal{D}^{*}[\alpha]$. Тогда $\mathscr{R}^{*} \varphi_{\mathcal{R} \mathcal{P}, \alpha}=\mathcal{F}_{\mathcal{R} \mathcal{P}}$.

3) Мы имеем $\varphi_{\varepsilon}: \mathcal{R} \mathcal{P} \rightarrow \mathcal{D}^{*}$. Тогда $\varphi_{\varepsilon}=\varphi_{\mathcal{R}, 0}$ и $\mathscr{R}^{*} \varphi_{\varepsilon}=\mathcal{F}_{\varepsilon}$.

УТВЕРЖДЕНИЕ 7.13. 1) Подгруппа $\varphi_{\mathcal{P}, \alpha}\left(\mathcal{P}^{2 n}\right) \subset \mathcal{D}^{*}[\alpha]_{-2 n}$ cocmoum uз всех однородных функиий $\psi(\alpha)$ степени $-2 n$ таких, что для любого $D \in \mathcal{D}$ выполнено условие $\langle\psi(-\alpha), \Phi(\alpha) D\rangle=\langle\psi(\alpha), D\rangle$.

2) Подгруппа $\varphi_{\mathcal{R} \mathcal{P}, \alpha}\left(\mathcal{R P}^{2 n}\right) \subset \mathcal{D}^{*}[\alpha]_{-2 n}$ coстоит из всех однородных функиий $\psi(\alpha)$ степени - 2n таких, что $\langle\psi(-\alpha), \Phi(\alpha) D\rangle=\langle\psi(0), D\rangle, D \in \mathcal{D}$.

3) Отображение $\varphi_{\varepsilon}: \mathcal{R P} \rightarrow \mathcal{D}^{*}$ является эпиморфизмом.

ДокАЗАТЕЛЬство. Согласно утверждению 1.68, мы имеем $\xi_{-\alpha} \Phi(\alpha)=\xi_{\alpha}$, поэтому если $\psi(\alpha)=\varphi_{\mathcal{P}, \alpha}(P)$, то

$$
\langle\psi(-\alpha), \Phi(\alpha) D\rangle=\xi_{-\alpha} \Phi(\alpha) D P=\xi_{\alpha} D P=\langle\psi(\alpha), D\rangle .
$$

$\mathrm{C}$ другой стороны, пусть для функции $\psi(\alpha)$ условие 1) выполнено. Тогда полином $g\left(\alpha, t_{1}, \ldots, t_{n}\right)=\mathscr{R}_{n}^{*} \psi(\alpha)$ удовлетворяет уравнению $(54)$ :

$$
\begin{aligned}
g\left(-\alpha, t_{1}, \ldots, t_{n-1}, \alpha\right) & =\left\langle\psi(-\alpha), \Phi(\alpha) \Phi\left(t_{n-1}\right) \cdots \Phi\left(t_{1}\right)\right\rangle \\
& =\left\langle\psi(\alpha), \Phi(0) \Phi\left(t_{n-1}\right) \cdots \Phi\left(t_{1}\right)\right\rangle=g\left(\alpha, t_{1}, \ldots, t_{n-1}, 0\right) .
\end{aligned}
$$


Из теоремы 7.3 следует, что он также удовлетворяет уравнениям (53), поэтому по теореме 6.8 мы получаем, что $g=\mathcal{F}_{\mathcal{P}, n}(P)$ для некоторого элемента $P \in \mathcal{P}^{2 n}$. Тогда $\mathscr{R}_{n}^{*} \psi(\alpha)=g=\mathcal{F}_{\mathcal{P}, n}(P)=\mathscr{R}_{n}^{*} \varphi_{\mathcal{P}, \alpha}(P)$. Так как отображение $\mathscr{R}^{*}$ является вложением и ограничение $\mathscr{R}^{*} \rightarrow \mathscr{R}_{n}^{*}$ изоморфно на группе $\mathcal{D}_{-2 j}^{*}$ при $j \leqslant n$, то $\psi(\alpha)=\varphi_{\mathcal{P}, \alpha}(P)$.

Точно так же доказывается утверждение 2). Утверждение 3) вытекает из следствия 7.5.

Имеется правое действие кольца $\mathcal{D}$ на кольце $\mathcal{D}^{*}$ :

$$
(\psi, D) \rightarrow \psi D: \quad\left\langle\psi D, D^{\prime}\right\rangle=\left\langle\psi, D D^{\prime}\right\rangle
$$

При этом если $\operatorname{deg} \psi=-2 n$ и $\operatorname{deg} D=-2 k$, то $\operatorname{deg} \psi D=-2(n-k)$. Подобным образом можно задать действие кольца $\mathcal{D}[[\alpha]]$ на кольце $\mathcal{D}^{*}[[\alpha]]$. Условие 1$)$ означает, что $\psi(-\alpha) \Phi(\alpha)=\psi(\alpha)$. Так как для любой функции $\psi(\alpha) \in \mathcal{D}^{*}[\alpha]_{-2 n}$ функция $\psi(\alpha) \Phi(\alpha)$ имеет ту же степень, то мы получаем следующий результат.

СлЕДСТвИЕ 7.14. Пусть $\psi(\alpha) \in \mathcal{D}^{*}[\alpha]$. Тогда:

1) $\psi(\alpha) \in \varphi_{\mathcal{P}, \alpha}(\mathcal{P})$ в том и только том случае, когда $\psi(-\alpha) \Phi(\alpha)=\psi(\alpha)$;

2) $\psi(\alpha) \in \varphi_{\mathcal{R P}, \alpha}(\mathcal{R P})$ в том и только том случае, когда $\psi(-\alpha) \Phi(\alpha)=\psi(0)$, m.е. $\psi(\alpha)=\psi(0) \Phi(\alpha)$. Поэтому соответствие $\psi \rightarrow \psi \Phi(\alpha)$ задает изоморфизм колеи, $\mathcal{D}^{*} \rightarrow \varphi_{\mathcal{R P}, \alpha}(\mathcal{R P})$.

ДокАЗАтЕльство. В доказательстве нуждается только тот факт, что отображение $\psi \rightarrow \psi \Phi(\alpha)$ является гомоморфизмом колец. Имеем $\varepsilon_{\mathcal{D}^{*}} \Phi(\alpha)=\varepsilon_{\mathcal{D}^{*}}$ и

$$
\begin{aligned}
& \left\langle\left(\psi_{1} \cdot \psi_{2}\right) \Phi(\alpha), D\right\rangle=\left\langle\left(\psi_{1} \cdot \psi_{2}\right), \Phi(\alpha) D\right\rangle=\left\langle\psi_{1} \otimes \psi_{2}, \Delta(\Phi(\alpha) D)\right\rangle \\
& \quad=\left\langle\psi_{1} \otimes \psi_{2},(\Phi(\alpha) \otimes \Phi(\alpha)) \cdot \Delta D\right\rangle=\left\langle\left(\psi_{1} \Phi(\alpha)\right) \otimes\left(\psi_{2} \Phi(\alpha)\right), \Delta D\right\rangle \\
& \quad=\left\langle\left(\psi_{1} \Phi(\alpha)\right) \cdot\left(\psi_{2} \Phi(\alpha)\right), D\right\rangle .
\end{aligned}
$$

УтВЕРЖДЕНИЕ 7.15. Для любого элемента $\psi(\alpha) \in \varphi_{\mathcal{P}, \alpha}(\mathcal{P})$ из равенства $\psi(0)=0$ cледует, что $\psi(\alpha)=\alpha^{2} \widehat{\psi}(\alpha)$, где $\widehat{\psi}(\alpha) \in \varphi_{\mathcal{P}, \alpha}(\mathcal{P})$.

ДокАзАТЕЛЬСтво. Из леммы 5.2 следует, что аддитивно $\mathcal{D}$ и $\mathcal{D}^{*}-$ свободные абелевы группы. Поэтому отображение $\mathcal{D}^{*} \rightarrow \mathcal{D}^{*} \otimes \mathbb{Q}$ является вложением. Так как $\mathcal{D}^{*} \otimes \mathbb{Q}[\alpha]$ - кольцо полиномов, то в кольце $\mathcal{D}^{*}[\alpha]$ нет делителей нуля. Из следствия 7.14 мы получаем, что $\left(\psi_{0}-\psi_{1} \alpha+\cdots\right)(1+d \alpha+\cdots)=\psi_{0}+\psi_{1} \alpha+\cdots$, поэтому $\psi_{0} d=2 \psi_{1}$. Следовательно, если $\psi_{0}=0$, то $\psi_{1}=0$. Тогда $\psi(\alpha)=$ $\alpha^{2} \widehat{\psi}(\alpha)$. При этом $(-\alpha)^{2} \widehat{\psi}(-\alpha) \Phi(\alpha)=\alpha^{2} \widehat{\psi}(\alpha)$. Тогда $\widehat{\psi}(-\alpha) \Phi(\alpha)=\widehat{\psi}(\alpha)$, поэтому $\widehat{\psi}(\alpha) \in \varphi_{\mathcal{P}, \alpha}(\mathcal{P})$. Утверждение доказано.

Пусть $\psi(\alpha)=\psi_{0}+\psi_{1} \alpha+\cdots+\psi_{n} \alpha^{n} \in \varphi_{\mathcal{P}, \alpha}(\mathcal{P}), u(\alpha)=\psi_{0}+\psi_{2} \alpha^{2}+\psi_{4} \alpha^{4}+\cdots$, $w(\alpha)=\psi_{1} \alpha+\psi_{3} \alpha^{3}+\cdots$. Тогда $u(\alpha) \Phi(\alpha)-w(\alpha) \Phi(\alpha)=u(\alpha)+w(\alpha)$. Следовательно,

$$
u(\alpha)(\Phi(\alpha)-1)=w(\alpha)(\Phi(\alpha)+1)
$$

Например, $\psi_{0} d=2 \psi_{1}, \psi_{0} d_{3}+\psi_{2} d=\psi_{1} d_{2}+2 \psi_{3}$. 
Рассмотрим алгебру Хопфа $\mathcal{D} \otimes \mathbb{Z}[1 / 2]$. Тогда для градуированно двойственной над кольцом $\mathbb{Z}[1 / 2]$ алгебры Хопфа мы имеем: $(\mathcal{D} \otimes \mathbb{Z}[1 / 2])^{*} \simeq \mathcal{D}^{*} \otimes \mathbb{Z}[1 / 2]$. Условие, описывающее образ кольца $\mathcal{P} \otimes \mathbb{Z}[1 / 2]$ в кольце $\mathcal{D}^{*} \otimes \mathbb{Z}[1 / 2][\alpha]$ - то же, а именно формула (64).

УтВеРЖДЕНИЕ 7.16. Для кольиа $\mathcal{D}^{*} \otimes \mathbb{Z}[1 / 2][\alpha]$ условие (64) эквивалентно соотношению

$$
w(\alpha)=u(\alpha) \frac{\Phi(\alpha)-1}{\Phi(\alpha)+1}=u(\alpha) \frac{(\Phi(\alpha)-1) / 2}{1+(\Phi(\alpha)-1) / 2}=u(\alpha) \sum_{k=1}^{\infty}(-1)^{k-1}\left(\frac{\Phi(\alpha)-1}{2}\right)^{k} .
$$

ЗАмЕчАНИЕ 7.17. Из уравнения (65) следует, что если функция $u(\alpha)$ четная, то функция $w(\alpha)$ является нечетной. Действительно,

$$
\begin{aligned}
w(-\alpha) & =u(-\alpha) \frac{\Phi(-\alpha)-1}{\Phi(-\alpha)+1}=u(\alpha) \frac{1 / \Phi(\alpha)-1}{1 / \Phi(\alpha)+1} \\
& =u(\alpha)(1-\Phi(\alpha)) \Phi(\alpha)^{-1}(1+\Phi(\alpha))^{-1} \Phi(\alpha)=-u(\alpha) \frac{\Phi(\alpha)-1}{\Phi(\alpha)+1}=-w(\alpha) .
\end{aligned}
$$

Поэтому для любой четной функции $u(\alpha)$ функция

$$
\psi(\alpha)=u(\alpha)+u(\alpha) \frac{\Phi(\alpha)-1}{\Phi(\alpha)+1}=u(\alpha) \frac{2 \Phi(\alpha)}{\Phi(\alpha)+1}
$$

принадлежит образу $\varphi_{\mathcal{P}, \alpha} \otimes 1(\mathcal{P} \otimes \mathbb{Z}[1 / 2]) \subset \mathcal{D}^{*} \otimes \mathbb{Z}[1 / 2][\alpha]$.

ЗАмечание 7.18. Соотношение (65) верно для кольца $\mathcal{D}^{*} \otimes \mathbb{Q}[\alpha]$.

Положим $\alpha=0$. Тогда мы получаем классическое отображение

$$
\varphi_{\mathcal{P}, 0}: \mathcal{P} \rightarrow \mathcal{D}^{*}, \quad\left\langle\varphi_{\mathcal{P}, 0}(P), D\right\rangle=\xi_{0}(D P) \quad \forall P \in \mathcal{P}, \quad D \in \mathcal{D} .
$$

УтвеРЖДЕНИЕ 7.19. Отображение $\varphi_{\mathcal{P}, 0} \otimes 1: \mathcal{P} \otimes \mathbb{Z}[1 / 2] \rightarrow \mathcal{D}^{*} \otimes \mathbb{Z}[1 / 2]$ сюрбективно.

ДоказАтельство. Пусть $\psi_{0} \in \mathcal{D}^{*} \otimes \mathbb{Z}[1 / 2]$. Рассмотрим элемент

$$
\psi(\alpha)=u(\alpha)+w(\alpha), \quad u(\alpha)=\psi_{0}, \quad w(\alpha)=\psi_{0} \frac{\Phi(\alpha)-1}{\Phi(\alpha)+1} .
$$

Согласно замечанию 7.17 , получаем $\psi(\alpha)=\left(\varphi_{\mathcal{P}, \alpha} \otimes 1\right) P \in\left(\varphi_{\mathcal{P}, \alpha} \otimes 1\right)(\mathcal{P} \otimes \mathbb{Z}[1 / 2])$. Тогда $\psi_{0}=\psi(0)=\left(\varphi_{\mathcal{P}, 0} \otimes 1\right)(P)$.

ЗАДАчА 7.20. Найти образ отображения $\varphi_{\mathcal{P}, 0}$ над кольцом иельх чисел.

ЗАмечАниЕ 7.21. Заметим, что абелева группа $\mathcal{P} / \operatorname{Ker} \varphi_{\mathcal{P}, \alpha}$ состоит из классов эквивалентности целочисленных комбинаций комбинаторных выпуклых многогранников по отношению эквивалентности: $P \sim Q$ тогда и только тогда, когда $P$ и $Q$ имеют одинаковые флаговые векторы. Ранг $2 n$-й градуированной компоненты этой группы равен числу Фибоначчи $c_{n}$ ([17], см. также п. 1.6).

С другой стороны, согласно следствию 5.8 мы имеем $\mathrm{rk}_{-2 n}^{*}=c_{n-1}$, поэтому отображение $\varphi_{\mathcal{P}, 0}: \mathcal{P} / \operatorname{Ker} \varphi_{\mathcal{P}, \alpha} \rightarrow \mathcal{D}^{*}$ не является мономорфизмом. 
ПримеР 7.22. Рассмотрим малые размерности.

(a) $n=1$. Группа $\mathcal{P} / \operatorname{Ker} \varphi_{\mathcal{P}, \alpha}$ порождена отрезком $C^{2}=I$. Аддитивная группа кольца $\mathcal{D}$ в этой градуировке порождена оператором $d$. Тогда $\varphi_{\mathcal{P}, 0}(I)=2 d^{*}$.

(b) $n=2$. Группа $\mathcal{P} / \operatorname{Ker} \varphi_{\mathcal{P}, \alpha}$ порождена многогранниками $C^{3}=\Delta^{2}$ и $B C^{2}=I^{2}$. Группа $\mathcal{D}$ в этой градуировке имеет базис, состоящий из оператора $d_{2}$. Тогда $\varphi_{\mathcal{P}, 0}\left(\Delta^{2}\right)=3 d_{2}^{*}, \varphi_{\mathcal{P}, 0}\left(I^{2}\right)=4 d_{2}^{*}$. Поэтому $d_{2}^{*}=\varphi_{\mathcal{P}, 0}\left(I^{2}-\Delta^{2}\right)$ и группа $\operatorname{Ker} \varphi_{\mathcal{P}, 0}$ свободно порождена элементом $3 I^{2}-4 \Delta^{2}$.

(c) $n=3$. Группа $\mathcal{P} / \operatorname{Ker} \varphi_{\mathcal{P}, \alpha}$ порождена многогранниками $B C^{3}, C B C^{2}=$ $C I^{2}, C^{4}=\Delta^{3}$, в то время как группа $\mathcal{D}$ порождена операторами $d_{3}, d_{2} d$. Тогда

$$
\begin{gathered}
\varphi_{\mathcal{P}, 0}\left(B C^{3}\right)=5 d_{3}^{*}+18\left(d_{2} d\right)^{*}, \quad \varphi_{\mathcal{P}, 0}\left(C B C^{2}\right)=5 d_{3}^{*}+16\left(d_{2} d\right)^{*}, \\
\varphi_{\mathcal{P}, 0}\left(C^{4}\right)=4 d_{3}^{*}+12\left(d_{2} d\right)^{*} .
\end{gathered}
$$

Поэтому $\operatorname{Im} \varphi_{\mathcal{P}, 0}$ имеет базис $d_{3}^{*}, 2\left(d_{2} d\right)^{*}$, a $\operatorname{Ker} \varphi_{\mathcal{P}, 0}$ имеет базис $2 B C^{3}-$ $6 C B C^{2}+5 C^{4}$.

Здесь через $D_{\omega}^{*}$ мы обозначаем элемент базиса, двойственного к базису

$$
\left\{d_{j_{1}} \cdots d_{j_{k}}, d_{j_{1}} \cdots d_{j_{k}} d, \quad k \geqslant 0, j_{i} \geqslant 2\right\}
$$

задаваемому разложением (44), т. е. $\left\langle D_{\omega}^{*}, D_{\sigma}\right\rangle=\delta_{\omega, \sigma}$.

\section{8. Мультипликативная структура колец флаговых векторов}

8.1. Основная теорема. Приведем основной результат данного раздела.

ТеОРема 8.1. $\times$-кольцо флаговых векторов $\mathcal{F}_{\mathcal{P}}(\mathcal{P}) \otimes \mathbb{Q} u$ *-кольцо фллаговых векторов $\mathcal{F}_{\varepsilon}(\mathcal{R P}) \otimes \mathbb{Q}$ являются градуированным кольиами полиномов, причем $\operatorname{dim} \mathcal{F}_{\mathcal{P}}\left(\mathcal{P}^{2 n}\right) \otimes \mathbb{Q}=c_{n} u \operatorname{dim} \mathcal{F}_{\varepsilon}\left(\mathcal{R P}^{2 n}\right) \otimes \mathbb{Q}=c_{n-1}$. Имеет место изоморфизм $\mathcal{F}_{\mathcal{P}}(\mathcal{P}) \otimes \mathbb{Q} \simeq \mathcal{F}_{\varepsilon}(\mathcal{R} \mathcal{P}) \otimes \mathbb{Q}\left[\alpha^{2}\right]$

ДокАЗАТЕЛЬСтво. Отождествим кольцо $\mathcal{D}^{*}$ с его образом $\mathscr{R}^{*}\left(\mathcal{D}^{*}\right) \subset \mathcal{Q} s y m$ и отображение $\varphi_{\mathcal{P}, \alpha}: \mathcal{P} \rightarrow \mathcal{D}^{*}$ с отображением $\mathscr{R}^{*} \varphi_{\mathcal{P}, \alpha}=\mathcal{F}_{\mathcal{P}}: \mathcal{P} \rightarrow \mathcal{Q}$ sym. Согласно следствию 7.5 , мы имеем $\mathcal{D}^{*}=\mathcal{F}_{\varepsilon}(\mathcal{R} \mathcal{P})$. Нам потребуется следующий результат.

Лемма 8.2. Пусть $\mathscr{F}=\left\{f_{\lambda}(\alpha) \in \mathcal{F}_{\mathcal{P}}(\mathcal{P}) \otimes \mathbb{Q}\right\}$ - набор однородных элементов. Положим $\mathscr{F}_{0}=\left\{f_{\lambda}=f_{\lambda}(0) \in \mathcal{D}^{*} \otimes \mathbb{Q}\right\}$. Тогда $\mathcal{F}_{\mathcal{P}}(\mathcal{P}) \otimes \mathbb{Q}=\mathbb{Q}\left[\mathscr{F}, \alpha^{2}\right]$ в том и только том случае, когда $\mathcal{D}^{*} \otimes \mathbb{Q}=\mathbb{Q}\left[\mathscr{F}_{0}\right]$.

ДоказАтельство. Пусть $\mathcal{D}^{*} \otimes \mathbb{Q}=\mathbb{Q}\left[\mathscr{F}_{0}\right]$. Докажем, что полиномы $\left\{f_{\lambda}(\alpha)\right\}$ и $\alpha^{2}$ алгебраически независимы.

Пусть $g \in \mathbb{Q}\left[x, y_{1}, \ldots, y_{s}\right]$ - такой полином, что $g\left(\alpha^{2}, f_{1}(\alpha), \ldots, f_{s}(\alpha)\right)=0$. Запишем это равенство в следующем виде:

$$
g_{0}\left(f_{1}(\alpha), \ldots, f_{s}(\alpha)\right)+g_{2}\left(f_{1}(\alpha), \ldots, f_{s}(\alpha)\right) \alpha^{2}+\cdots+g_{2 t}\left(f_{1}(\alpha), \ldots, f_{s}(\alpha)\right) \alpha^{2 t}=0
$$

для некоторого $t \geqslant 0$. Положим $\alpha=0$. Тогда $g_{0}\left(f_{1}, \ldots, f_{s}\right)=0$. Поскольку элементы $\left\{f_{\lambda}\right\}$ алгебраически независимы, то $g_{0} \equiv 0$. Так как кольцо $\mathcal{D}^{*} \otimes \mathbb{Q}[\alpha]$ является кольцом полиномов, то мы можем разделить равенство (66) на $\alpha^{2}$. Тогда

$g_{2}\left(f_{1}(\alpha), \ldots, f_{s}(\alpha)\right)+g_{4}\left(f_{1}(\alpha), \ldots, f_{s}(\alpha)\right) \alpha^{2}+\cdots+g_{2 t}\left(f_{1}(\alpha), \ldots, f_{s}(\alpha)\right) \alpha^{2 t-2}=0$. 
Повторяя это рассуждение, мы в итоге получим, что все полиномы $g_{2 i}, i=$ $0,1, \ldots, t$, равны нулю, поэтому $g \equiv 0$.

Теперь покажем, что полиномы $\left\{f_{\lambda}(\alpha)\right\}$ и $\alpha^{2}$ порождают кольцо $\mathcal{F}_{\mathcal{P}}(\mathcal{P}) \otimes \mathbb{Q}$. Пусть $\psi(\alpha)=\psi_{0}+\psi_{1} \alpha+\cdots+\psi_{n} \alpha^{n} \in \mathcal{F}_{\mathcal{P}}\left(\mathcal{P}^{2 n}\right) \otimes \mathbb{Q}$. Функции $\left\{f_{\lambda}\right\}$ порождают кольцо $\mathcal{D}^{*} \otimes \mathbb{Q}$, поэтому существует однородный полином $g_{0} \in \mathbb{Q}\left[y_{1}, \ldots, y_{s}\right]$ такой, что $g_{0}\left(f_{1}, \ldots, f_{s}\right)=\psi_{0}, \operatorname{deg} f_{i} \leqslant 2 n$.

Рассмотрим элемент $\psi(\alpha)-g_{0}\left(f_{1}(\alpha), \ldots, f_{s}(\alpha)\right) \in \mathcal{F}_{\mathcal{P}}\left(\mathcal{P}^{2 n}\right) \otimes \mathbb{Q}$. Из рациональной версии утверждения 7.15 получаем, что $\psi(\alpha)-g_{0}\left(f_{1}(\alpha), \ldots, f_{s}(\alpha)\right)=$ $\widehat{\psi}(\alpha) \alpha^{2}$, где $\widehat{\psi}(\alpha) \in \mathcal{F}_{\mathcal{P}}\left(\mathcal{P}^{2(n-2)}\right) \otimes \mathbb{Q}$.

Повторяя это рассуждение, мы в конце концов получим выражение функции $\psi(\alpha)$ в виде полинома от функций $\left\{f_{\lambda}(\alpha)\right\}$ и $\alpha^{2}$.

Пусть теперь $\mathcal{D}^{*} \otimes \mathbb{Q}=\mathbb{Q}\left[\mathscr{F}, \alpha^{2}\right]$. Докажем, что полиномы $\left\{f_{\lambda}\right\}$ алгебраически независимы. Пусть $g \in \mathbb{Q}\left[y_{1}, \ldots, y_{k}\right]$ - такой полином, что $g\left(f_{1}, \ldots, f_{s}\right)=0$. Из рациональной версии утверждения 7.15 следует, что $g\left(f_{1}(\alpha), \ldots, f_{s}(\alpha)\right)=$ $\alpha^{2} \widehat{g}$, где $\widehat{g} \in \mathcal{F}_{\mathcal{P}}(\mathcal{P}) \otimes \mathbb{Q}$. Мы имеем $\widehat{g}=p\left(\alpha^{2}, f_{1}(\alpha), \ldots, f_{t}(\alpha)\right)$ для некоторого полинома $p\left(x, y_{1}, \ldots, y_{t}\right) \in \mathbb{Q}\left[x, y_{1}, \ldots, y_{t}\right]$. Если $g \neq 0$, то $p \neq 0$ и мы получаем алгебраическую зависимость между элементами $\alpha^{2}$ и $\left\{f_{\lambda}(\alpha)\right\}$. Следовательно, $g \equiv 0$. Поэтому элементы из множества $\mathscr{F}_{0}$ алгебраически независимы.

Из рациональной версии утверждения 7.19 следует, что для любого элемента $\psi \in \mathcal{D}^{*} \otimes \mathbb{Q}$ найдется такой элемент $P \in \mathcal{P} \otimes \mathbb{Q}$, что $\psi=\left(\varphi_{\mathcal{P}, 0} \otimes 1\right) P$. Следовательно, $\psi=\left.\left(\mathcal{F}_{\mathcal{P}} \otimes 1\right)(P)\right|_{\alpha=0}$. Но $\left(\mathcal{F}_{\mathcal{P}} \otimes 1\right)(P)=p\left(\alpha, f_{1}(\alpha), \ldots, f_{t}(\alpha)\right)$ для некоторого полинома $p \in \mathbb{Q}\left[x, y_{1}, \ldots, y_{t}\right]$, поэтому $\psi=p\left(0, f_{1}, \ldots, f_{t}\right)$.

Таким образом, элементы $\left\{f_{\lambda}\right\}$ порождают кольцо $\mathcal{D}^{*} \otimes \mathbb{Q}$. Следовательно, $\mathcal{D}^{*} \otimes \mathbb{Q}=\mathbb{Q}\left[\mathscr{F}_{0}\right]$. Лемма доказана.

Вернемся к доказательству теоремы. Согласно утверждению 7.9 , имеем $\mathcal{D}^{*} \otimes$ $\mathbb{Q} \simeq \mathbb{Q}\left[\mathrm{LYN}_{\text {odd }}\right]$, где $\mathbb{Q}\left[\mathrm{LYN}_{\text {odd }}\right]$, поэтому $\mathcal{D}^{*} \otimes \mathbb{Q}[\alpha]$ - кольцо полиномов от слов Линдона $\mathrm{LYN}_{\text {odd }}$ и переменной $\alpha$. Пусть $\left\{f_{\lambda} \in \mathcal{D}^{*} \otimes \mathbb{Q}\right\}$ - набор базисных функций, отвечающих словам Линдона. Положим

$$
f_{\lambda}(\alpha)=u_{\lambda}(\alpha)+w_{\lambda}(\alpha), \quad u_{\lambda}(\alpha)=f_{\lambda}, \quad w_{\lambda}(\alpha)=f_{\lambda} \frac{\Phi(\alpha)-1}{\Phi(\alpha)+1}=f_{\lambda} \frac{e^{s(\alpha)}-1}{e^{s(\alpha)}+1} .
$$

Каждый полином $f_{\lambda}(\alpha)$ является однородным степени $\operatorname{deg} f_{\lambda}$. Согласно замечаниям 7.17 и 7.18 , эти полиномы принадлежат образу $\left(\mathcal{F}_{\mathcal{P}} \otimes 1\right)(\mathcal{P} \otimes \mathbb{Q})$. При этом $f_{\lambda}(0)=f_{\lambda}$.

Теперь теорема следует из леммы 8.2 и равенства $\mathcal{F}_{\varepsilon}(\mathcal{R} \mathcal{P})=\mathscr{R}^{*}\left(\mathcal{D}^{*}\right)$.

ЗАМЕчАНИЕ 8.3. Из доказательства теоремы 8.1 следует, что композиция $\mathcal{F}_{\mathcal{P}}(\mathcal{P}) \otimes \mathbb{Q} \simeq \mathcal{F}_{\varepsilon}(\mathcal{R} \mathcal{P}) \otimes \mathbb{Q}\left[\alpha^{2}\right] \stackrel{\alpha=0}{\longrightarrow} \mathcal{F}_{\varepsilon}(\mathcal{R} \mathcal{P}) \otimes \mathbb{Q}$ имеет вид $\left.\mathcal{F}_{\mathcal{P}}(P) \rightarrow \mathcal{F}_{\mathcal{P}}(P)\right|_{\alpha=0}$, a ее ядро порождается полиномом $\alpha^{2}$. Она описывается геометрически. Рассмотрим отображение $l_{0}: \mathcal{P} \rightarrow \mathcal{R} \mathcal{P}$, задаваемое формулой $l_{0}(P)=\sum_{v \subseteq P} P / v$. Тогда $l_{0}$ - кольцевой гомоморфизм и $\mathcal{F}_{\varepsilon}\left(l_{0}(P)\right)=\left.\mathcal{F}_{\mathcal{P}}(P)\right|_{\alpha=0}$ для любого $P \in \mathcal{P}$.

СлЕДСТВИЕ 8.4. Имеет место изоморфизм градуированных алгебр полиномов:

$$
\mathcal{F}_{\mathcal{P}}(\mathcal{P}) \otimes \mathbb{Q} \simeq \mathbb{Q}\left[\mathrm{LYN}_{\text {odd }}, \alpha^{2}\right]=\mathcal{N}_{\text {odd }} \otimes \mathbb{Q}\left[\alpha^{2}\right] \simeq \mathbb{Q}\left[\operatorname{LYN}_{\{12\}}\right]=\mathcal{N}_{\{12\}} \otimes \mathbb{Q}
$$


СЛЕДСТВИЕ 8.5. Множество $\mathscr{F}_{\times}^{2 n}$ из $k_{n}$ элементов группъ $\mathcal{F}_{\mathcal{P}}\left(\mathcal{P}^{2 n}\right) \otimes \mathbb{Q}$ является набором $2 n$-мерных мультипликативных образующих $\times$-кольца флаговых векторов тогда и только тогда, когда

1) $f_{\{0\}} \neq 4 f_{\varnothing} \partial \Omega_{\Omega} \mathscr{F}_{\times}^{4}=\left\{f_{\varnothing} \alpha^{2}+f_{\{1\}} \alpha \sigma^{1}+f_{\{0\}} \sigma_{1}^{2}\right\}$,

2) для $n \neq 2$ множество $\mathscr{F}_{0}^{2 n}$ из $k_{n}$ элементов группы $\mathcal{D}_{-2 n}^{*} \otimes Q$ вида $\left\{f_{n}(0): f_{n}(\alpha) \in \mathscr{F}_{\times}^{2 n}\right\}$ является набором мультипликативньх образующих кольиа $\mathcal{D}^{*} \otimes \mathbb{Q}$.

ДокАЗАТЕЛЬство. Элемент

$$
f_{\varnothing} \alpha^{2}+f_{\{1\}} \alpha \sigma_{1}+f_{\{0\}} \sigma_{1}^{2}=\frac{1}{4}\left(\left(4 f_{\varnothing}-f_{\{0\}}\right) \alpha^{2}+f_{\{0\}}\left(\alpha+2 \sigma_{1}\right)^{2}\right)
$$

является мультипликативной образующей тогда и только тогда, когда выполнено условие $f_{\{0\}} \neq 4 f_{\varnothing}$.

Для $n \neq 2$ доказательство вытекает из леммы 8.2.

\section{2. Мультипликативные образующие.}

ОПРЕДЕЛЕНИЕ 8.6. Введем оператор

$$
\begin{aligned}
\pi(t)=\sum_{n \geqslant 1} \pi_{n} t^{n-1} & =\Phi(-t) \frac{\partial}{\partial t} \Phi(t)=\Phi(-t) \Phi^{\prime}(t) \\
& =\sum_{n \geqslant 1}\left(\sum_{i=0}^{n-1}(-1)^{i}(n-i) d_{i} d_{n-i}\right) t^{n-1} .
\end{aligned}
$$

Для любых элементов $P, Q \in \mathrm{R}$ имеем $\Phi(t)(P Q)=(\Phi(t) P) \cdot(\Phi(t) Q)$, поэтому

$$
\Phi^{\prime}(t)(P Q)=\left(\Phi^{\prime}(t) P\right) \cdot(\Phi(t) Q)+(\Phi(t) P) \cdot\left(\Phi(t)^{\prime} Q\right)
$$

и $\pi(t)(P Q)=(\pi(t) P) Q+P(\pi(t) Q)$. Следовательно, каждый оператор $\pi_{n}(t)$ является дифференцированием.

УТВЕРЖДЕНИЕ 8.7. $\mathcal{D} \otimes \mathbb{Q} \simeq \mathbb{Q}\left\langle\pi_{1}, \pi_{3}, \pi_{5}, \ldots\right\rangle$.

ДокАЗАтельство. Имеем: $\pi_{1}=d, \pi_{2}=2 d_{2}-d^{2}$,

$$
\pi_{n}=n d_{n}-(n-1) d d_{n-1}+\cdots+(-1)^{n-1} d_{n-1} d .
$$

Используя формулу (67) и соотношения (19), индукцией по $k$ получаем, что операторы $d_{2 k-1}$ являются некоммутативными полиномами от $\pi_{2 l-1}, l \leqslant k$. Следовательно, требуемый изоморфизм вытекает из утверждения 5.9.

Из общей теории алгебр Ли (см. [33]) следует, что линейное пространство $\operatorname{Prim}\left(\mathbb{Q}\left\langle\pi_{1}, \pi_{3}, \ldots\right\rangle\right)$ примитивных элементов алгебры Хопфа $\mathbb{Q}\left\langle\pi_{1}, \pi_{3}, \ldots\right\rangle$ является свободной алгеброй Ли от образующих $\pi_{1}, \pi_{3}, \pi_{5}, \ldots$ Аддитивный базис в этом пространстве описывается рекуррентно в терминах слов Линдона.

ОПРЕДЕЛЕНИЕ 8.8. Для слова Линдона $w=\left[a_{1}, \ldots, a_{k}\right]$ обозначим через $w^{\prime \prime}=\left[a_{i}, a_{i+1}, \ldots, a_{k}\right]$ максимальный по длине собственный конец этого слова, который является словом Линдона. Положим $w^{\prime}=\left[a_{1}, \ldots, a_{i-1}\right]$.

УтВЕРЖДЕНИЕ 8.9. (i) Слово $w^{\prime \prime}$ является минимальным из всех собственных кониов слова $w$.

(ii) Слово $w^{\prime}$ является словом Линдона.

СлеДСтвиЕ 8.10. Имеет место каноническое разложение $w=w^{\prime} * w^{\prime \prime}$ такое, что $w^{\prime}, w^{\prime \prime}$ - слова Линдона $и w^{\prime}<w<w^{\prime \prime}$. 
Положим $\pi_{w}=\left[\pi_{w^{\prime}}, \pi_{w^{\prime \prime}}\right]=\pi_{w^{\prime}} \pi_{w^{\prime \prime}}-\pi_{w^{\prime \prime}} \pi_{w^{\prime}}$, где $w \in \mathrm{LYN}_{\text {odd }}$ и $\pi_{[2 k-1]}=\pi_{2 k-1}$. Например,

$\pi_{[1,3]}=\left[\pi_{[1]}, \pi_{[3]}\right]=3\left(d d_{3}-d_{3} d\right), \quad \pi_{[1,1,3]}=\left[\pi_{[1]}, \pi_{[1,3]}\right]=3\left(d^{2} d_{3}-2 d d_{3} d+d_{3} d^{2}\right)$.

УтВЕРЖДЕНИЕ 8.11. Операторы $\pi_{w}, w \in \mathrm{LYN}_{\mathrm{odd}}$, образуют базис линейного пространства $\operatorname{Prim}(\mathcal{D} \otimes \mathbb{Q})$.

Доказательство вытекает из утверждения 8.7 и теории свободных алгебр Ли [33].

Теорема 8.12. (i) Множество $T_{\times}^{2 n}$ из $k_{n}$ элементов группъ $\mathcal{P}^{2 n} \otimes \mathbb{Q}$ задает набор 2 -мерных мультипликативных образующих $\times$-кольца флаговых векторов тогда и только тогда, когда

1) $\left(\xi_{0} d_{2}-4 \xi_{1}\right) P^{2} \neq 0$ для $T_{\times}^{4}=\left\{P^{2}\right\}$,

2) для $n \neq 2$ матрииа $\Pi^{n}=\left\{\Pi_{Q, w}^{n}=\xi_{0}\left(\pi_{w} Q\right)\right\} \in \operatorname{Mat}_{k_{n} \times k_{n}}(\mathbb{Q})$, где $Q \in T_{\times}^{2 n} u w \in \mathrm{LYN}_{\text {odd }}^{2 n}$, невырождена.

(ii) Множество $T_{*}^{2 n}, n \neq 2$, из $k_{n}$ элементов группы $\mathcal{R P}^{2 n} \otimes \mathbb{Q}$ задает набор мультипликативных образующих *-кольца флаговых векторов тогда u только тогда, когда матрица $\Pi^{n}=\left\{\Pi_{Q, w}^{n}=\varepsilon\left(\pi_{w} Q\right)\right\} \in \operatorname{Mat}_{k_{n} \times k_{n}}(\mathbb{Q})$, где $Q \in T_{*}^{2 n} u w \in \mathrm{LYN}_{\mathrm{odd}}^{2 n}$, невырождена.

ДоказАтельство. Имеем: $\left(\xi_{0} d_{2}-\xi_{1}\right) P^{2}=f_{\{0\}}\left(P^{2}\right)-4 f_{\varnothing}\left(P^{2}\right)$. Из следствия 8.5 вытекает, что в случае $n=2$ множество $T_{\times}^{4}$ состоит из мультипликативной образующей тогда и только тогда, когда выполнено условие 1 ), а в случае $n \neq 2$ множество $T_{\times}^{2 n}$ задает набор мультипликативных образующих тогда и только тогда, когда набор $\mathscr{F}_{0}^{2 n}=\left\{\left(\varphi_{\mathcal{P}, 0} \otimes 1\right) P^{n}: P^{n} \in T_{\times}^{2 n}\right\}$ является набором мультипликативных образующих кольца $\mathcal{D}^{*} \otimes \mathbb{Q}$.

Согласно теории градуированных алгебр Хопфа, мультипликативные образующие алгебры Хопфа соответствуют примитивным элементам градуированно двойственной алгебры Хопфа, т. е. имеет место следующий факт.

Лемма 8.13. Множество $\mathscr{F}_{0}^{2 n}, n \neq 2$, из $k_{n}$ элементов группъ $\mathcal{D}_{-2 n}^{*} \otimes \mathbb{Q}$ является набором (-2n)-мерных мультипликативных образующих кольца $\mathcal{D}^{*} \otimes \mathbb{Q}$ тогда и только тогда, когда матриц, $\Pi^{n}=\left\{\Pi_{f_{\lambda}, w}^{n}=\left\langle f_{\lambda}, \pi_{w}\right\rangle\right\} \in$ $\operatorname{Mat}_{k_{n} \times k_{n}}(\mathbb{Q})$, где $f_{\lambda} \in \mathscr{F}_{0}^{2 n} u w \in \mathrm{LYN}_{\text {odd }}^{2 n}$, невырождена.

Имеем: $\left\langle\left(\varphi_{\mathcal{P}, 0} \otimes 1\right) P, \pi_{w}\right\rangle=\xi_{0} \pi_{w} P$, откуда следует первое утверждение теоремы.

Пользуясь отождествлением $\mathcal{D}^{*}=\mathscr{R}^{*}\left(\mathcal{D}^{*}\right)$, получаем

$$
\left\langle\mathcal{F}_{\varepsilon}(P), \pi_{w}\right\rangle=\left\langle\varphi_{\varepsilon}(P), \pi_{w}\right\rangle=\varepsilon\left(\pi_{w} P\right),
$$

откуда следует второе утверждение теоремы.

\section{9. Комодули Хопфа}

Пусть $R$ - поле или кольцо целых чисел $\mathbb{Z}$.

ОПРЕДЕЛЕНИЕ 9.1. (Левым) комодулем Хопфа над алгеброй Хопфа $X$ называется $R$-алгебра $M$ с единицей такая, что $M$ является комодулем над $X$, кодействие $b: M \rightarrow X \otimes M$ которого является гомоморфизмом колец. 
9.1. Модули и комодули Хопфа. Приведем необходимые нам факты из общей теории алгебр Хопфа, модулей и комодулей над ними. Доказательство этих фактов непосредственно вытекает из определений.

Лемма 9.2. Пусть $\mathcal{H}$ - связная градуированная $\mathbb{Z}$-алгебра Хопфа без кручения такая, что $\mathrm{rk} \mathcal{H}^{n}<\infty, n \geqslant 0$. Пусть кольцо $\mathcal{A}$ имеет структуру правого (левого) градуированного $\mathcal{H}$-модуля Хопфа. Тогда кольио $\mathcal{A}$ имеет структуру левого (правого) градуированного $\mathcal{H}^{*}$-комодуля Хопфа, определяемую формулой

$$
\Delta_{\mathcal{H}^{*}}(a)=\sum_{\omega} H_{\omega}^{*} \otimes\left(a H_{\omega}\right) \quad\left(\Delta_{\mathcal{H}^{*}}(a)=\sum_{\omega}\left(H_{\omega} a\right) \otimes H_{\omega}^{*}\right),
$$

где $\left\{H_{\omega}\right\}$ - некоторый базис градуированной группы $\mathcal{H} u\left\{H_{\omega}^{*}\right\}-$ двойственный базис градуированно двойственной группы: $\left\langle H_{\omega}^{*}, H_{\sigma}\right\rangle=\delta_{\omega, \sigma}$.

ЗАмечАниЕ 9.3. Отображение $\Delta_{\mathcal{H}}$ не зависит от выбора базиса в градуированной группе $\mathcal{H}$, так как мы можем записать его в виде

$$
\Delta_{\mathcal{H}^{*}}(a)=(1 \otimes a)\left(\sum_{\omega} H_{\omega}^{*} \otimes H_{\omega}\right)
$$

и элемент $\sum H_{\omega}^{*} \otimes H_{\omega}$ определяет на каждой градуированной компоненте $\mathcal{H}^{n}$ тождественный оператор как элемент группы $\left(\mathcal{H}^{n}\right)^{*} \otimes \mathcal{H}^{n} \simeq \operatorname{Hom}_{\mathbb{Z}}\left(\mathcal{H}^{n}, \mathcal{H}^{n}\right)$.

СледСтвиЕ 9.4. На кольце $\mathrm{R}$ имеется естественная структура левого градуированного $\mathcal{Q}$ sут-комодуля Хопфа, индуцированная структурой правого градуированного $\mathcal{Z}$-модуля Хопфа:

$$
\begin{aligned}
\Delta_{\mathscr{R}}(P) & =\sum_{\omega} M_{\omega} \otimes P Z_{\omega}=\sum_{k \geqslant 0} \sum_{\left(j_{1}, \ldots, j_{k}\right)} M_{\left(j_{1}, \ldots, j_{k}\right)} \otimes\left(P Z_{j_{1}} \cdots Z_{j_{k}}\right) \\
& =\sum_{k \geqslant 0} \sum_{\left(j_{1}, \ldots, j_{k}\right)} M_{\left(j_{k}, \ldots, j_{1}\right)} \otimes\left(d_{j_{1}} \cdots d_{j_{k}} P\right) .
\end{aligned}
$$

На кольще $\mathrm{R}$ имеются естественные структури $\Delta_{\mathscr{L}}$ u $\Delta_{\mathcal{D}^{*}}$ правого градуированного комодуля Милнора над алгебрами Хопфа $\mathcal{Q}$ sym и $\mathcal{D}^{*}$ соответственно, индуцированные структурами левого градуированного модуля Милнора над алгебрами Хопфа $\mathcal{Z}$ и $\mathcal{D}$. Эти структуры согласованы в следующем смысле:

$$
\begin{gathered}
\Delta_{\mathscr{L}}=\tau_{\mathcal{Q} s y m, \mathrm{R}}(* \otimes 1) \Delta_{\mathscr{R}}, \\
\left(1 \otimes \mathscr{L}^{*}\right) \Delta_{\mathcal{D}^{*}}=\Delta_{\mathscr{L}} .
\end{gathered}
$$

Отображение $\Delta_{\mathscr{L}}$ задается формулой

$$
\Delta_{\mathscr{L}} P=\sum_{k \geqslant 0} \sum_{\left(j_{1}, \ldots, j_{k}\right)}\left(d_{j_{1}} \cdots d_{j_{k}} P\right) \otimes M_{\left(j_{1}, \ldots, j_{k}\right)} .
$$

ДокАзАтельство. Существование этих структур и явные формулы для них непосредственно следуют из леммы 9.2. Соотношение (69) легко следует из формул для отображений $\Delta_{\mathscr{L}}$ и $\Delta_{\mathscr{R}}$.

Так как $\mathcal{D} \simeq \mathcal{U} \simeq \mathcal{Z} / J_{\mathcal{U}}-$ свободная абелева группа, то $\mathcal{Z}=\mathcal{Z}^{\prime} \oplus J_{U}$. Выберем базис в градуированных группах: $\left\{H_{\beta}\right\}$ в $\mathcal{Z}^{\prime}$ и $\left\{H_{\gamma}\right\}$ в $J_{\mathcal{U}}$. Тогда элементы $\left\{H_{\beta}, H_{\gamma}\right\}$ образуют базис в градуированной группе $\mathcal{Z}$. Обозначим через $\left\{H_{\beta}^{*}\right\}$, $\left\{H_{\gamma}^{*}\right\}$ элементы двойственного базиса в градуированной группе $\mathcal{Q} s y m$. 
Заметим, что $\mathscr{L}^{*}\left(\mathcal{D}^{*}\right)=\left\{\psi \in \mathcal{Q}\right.$ sym $\left.:\left.\psi\right|_{J_{\mathcal{U}}}=0\right\}$, причем функции $\left\{H_{\beta}^{*}\right\}$ образуют базис в этой группе. Пусть $\left\{D_{\beta}^{*} \in \mathcal{D}^{*}\right\}$ - такие функции, что $H_{\beta}^{*}=\mathscr{L}^{*} D_{\beta}^{*}$. Тогда операторы $\left\{D_{\beta}=\mathscr{L} H_{\beta}\right\}$ образуют двойственный базис в градуированной группе $\mathcal{D}$.

Для любого элемента $P \in \mathrm{R}$ мы имеем:

$$
\Delta_{\mathscr{L}} P=\sum_{\beta}\left(H_{\beta} P\right) \otimes H_{\beta}^{*}+\sum_{\gamma}\left(H_{\gamma} P\right) \otimes H_{\gamma}^{*}
$$

Но $H_{\gamma} P=0$ для всех $\gamma$, поэтому

$\Delta_{\mathscr{L}} P=\sum_{\beta} H_{\beta} P \otimes H_{\beta}^{*}=\sum_{\beta}\left(\mathscr{L} H_{\beta}\right) P \otimes H_{\beta}^{*}=\sum_{\beta} D_{\beta} P \otimes \mathscr{L}^{*} D_{\beta}^{*}=\left(1 \otimes \mathscr{L}^{*}\right) \Delta_{\mathcal{D}^{*}} P$,

что и доказывает следствие.

Из сопоставления формул (48) и (68) мы получаем следующее утверждение.

УтВЕРЖДЕНИЕ 9.5. Имеем: $\Phi_{\infty}=\Delta_{\mathscr{R}}$.

9.2. Каноническая структура на $\times$-кольце многогранников. Коумножение $\Delta$ на алгебре Хопфа $\mathcal{R} \mathcal{P}$ не индуцирует коумножения на кольце $\mathcal{P}$. Однако кольцо $\mathcal{P}$ является правым комодулем Хопфа над $\mathcal{R} \mathcal{P}$.

УТВЕРЖДЕНИЕ 9.6. Формула

$$
\Delta_{\mathcal{R} \mathcal{P}} P=\sum_{F \subseteq P} F \otimes(P / F)
$$

определяет каноническое кодействие $\Delta_{\mathcal{R}}: \mathcal{P} \rightarrow \mathcal{P} \otimes \mathcal{R} \mathcal{P}$ правого комодуля Хопфа над $\mathcal{R} \mathcal{P}$.

ДокАЗАТЕльство. Следующие две диаграммы коммутативны:
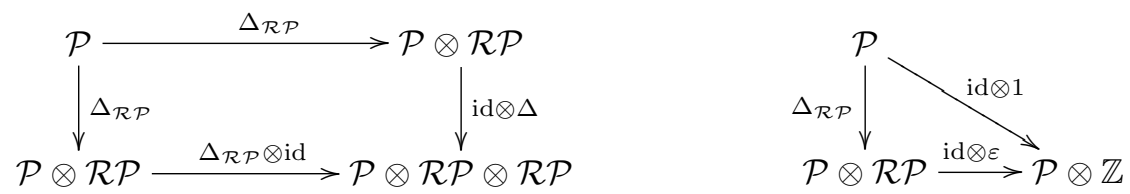

Действительно,

$$
\begin{aligned}
\left(\Delta_{\mathcal{R P}}\right. & \otimes \mathrm{id}) \Delta_{\mathcal{R} \mathcal{P}} P=\left(\Delta_{\mathcal{R} \mathcal{P}} \otimes \mathrm{id}\right)\left(\sum_{F \subseteq P} F \otimes P / F\right) \\
& =\sum_{F \subseteq P}\left(\sum_{G \subseteq F} G \otimes F / G\right) \otimes P / F=\sum_{G \subseteq P} G \otimes\left(\sum_{G \subseteq F \subseteq P} F / G \otimes P / F\right) \\
& =\sum_{G \subseteq P} G \otimes \Delta(P / G)=(\mathrm{id} \otimes \Delta) \Delta_{\mathcal{R} \mathcal{P} P}
\end{aligned}
$$

и $(\mathrm{id} \otimes \varepsilon) \Delta_{\mathcal{R} \mathcal{P}}(P)=\sum_{F \subseteq P} F \otimes \varepsilon(P / F)=P \otimes 1$. 
Теперь покажем, что кодействие $\Delta_{\mathcal{R}}$ является мультипликативным:

$$
\begin{aligned}
\Delta_{\mathcal{R P}}(P \times Q) & =\sum_{F \times G \subseteq P \times Q}(F \times G) \otimes(P \times Q / F \times G) \\
& =\sum_{F \subseteq P, G \subseteq Q}(F \times G) \otimes(P / F * Q / G) \\
& =\left(\sum_{F \subseteq P} F \otimes P / F\right) \cdot\left(\sum_{G \subseteq Q} G \otimes Q / G\right)=\Delta_{\mathcal{R P}}(P) \cdot \Delta_{\mathcal{R P}}(Q) \\
\Delta_{\mathcal{R P}}(\mathrm{pt}) & =\mathrm{pt} \otimes(\mathrm{pt} / \mathrm{pt})=\mathrm{pt} \otimes \varnothing=1 \otimes 1 .
\end{aligned}
$$

СлеДСТВИе 9.7. Любая пара $\eta: \mathcal{P} \rightarrow R_{1}, \zeta: \mathcal{R P} \rightarrow R_{2}$ линейных гомоморфизмов индуиирует линейный гомоморфизм $\eta \star \zeta: \mathcal{P} \rightarrow R_{1} \otimes R_{2}$ :

$$
(\eta \star \zeta)(P)=(\eta \otimes \zeta) \Delta_{\mathcal{R} \mathcal{P}}(P)=\sum_{F \subseteq P} \eta(F) \otimes \zeta(P / F),
$$

который называется их сверткой. Если $R_{1}$ и $R_{2}$ - кольца и $\eta, \zeta$ - гомоморфизмы колеи, то $\eta \star \zeta-$ гомоморфизм колец. В частности, любой гомоморфизм $\zeta: \mathcal{R P} \rightarrow \mathbb{Z}$ задает линейньй оператор id $\star \zeta \in \mathcal{L}(\mathcal{P})$.

ПримеР 9.8. $\alpha$-характер $\xi_{\alpha}: \mathcal{P} \rightarrow \mathbb{Z}[\alpha]$ определяет кольцевой гомоморфизм $l_{\alpha}: \mathcal{P} \rightarrow \mathcal{R} \mathcal{P}[\alpha]$ такой, что $l_{\alpha}=\xi_{\alpha} \star 1: l_{\alpha}\left(P^{n}\right)=\sum \alpha^{\operatorname{dim} F} P / F, F \subseteq P$.

В случае простых многогранников любая гранная фигура $i$-мерной грани является симплексом $\Delta^{n-i-1}=x^{n-i}$. Поэтому

$$
l_{\alpha}\left(P^{n}\right)=\sum_{i=0}^{n} f_{\{i\}} \alpha^{i} x^{n-i}=\mathcal{F}_{\mathcal{P}, 1}(\alpha, x)\left(P^{n}\right)=f(\alpha, x)\left(P^{n}\right) .
$$

УтВеРЖДЕНИЕ 9.9. Для любых двух элементов $P, Q \in \mathcal{P}$

$$
\Delta_{\mathcal{R P}}(P * Q)=(1 \otimes P) \cdot \Delta_{\mathcal{R} \mathcal{P}}(Q)+\Delta_{\mathcal{R P}}(P) \cdot(1 \otimes Q)+\Delta_{\mathcal{R} \mathcal{P}}(P)(* \otimes *) \Delta_{\mathcal{R P}}(Q) .
$$

ДокАЗАТЕЛЬСтво. Действительно,

$$
\begin{gathered}
\Delta_{\mathcal{R P}}(P * Q)=\sum_{G \subseteq Q}(\varnothing * G) \otimes(P * Q / \varnothing * G)+\sum_{F \subseteq P}(F * \varnothing) \otimes(P * Q / F * \varnothing) \\
\quad+\sum_{F \subseteq P, G \subseteq Q}(F * G) \otimes(P * Q / F * G)=\sum_{G \subseteq Q} G \otimes(P *(Q / G)) \\
\quad+\sum_{F \subseteq P} F \otimes((P / F) * Q)+\sum_{F \subseteq P, G \subseteq Q}(F * G) \otimes(P / F * Q / G) \\
=(1 \otimes P) \Delta_{\mathcal{R P}}(Q)+\Delta_{\mathcal{R P}}(P)(1 \otimes Q)+\Delta_{\mathcal{R P}}(P)(* \otimes *) \Delta_{\mathcal{R} \mathcal{P}}(Q) .
\end{gathered}
$$

СлеДСтвиЕ 9.10. Для любых двух элементов $P, Q \in \mathcal{P}$

$$
l_{\alpha}(P * Q)=P * l_{\alpha}(Q)+l_{\alpha}(P) * Q+\alpha l_{\alpha}(P) \cdot l_{\alpha}(Q) .
$$

ДоказАтельство. Применим гомоморфизм $\xi_{\alpha} \otimes 1$ к формуле (74).

9.3. Комодульные структуры и гомоморфизмы. В этом пункте мы установим взаимосвязь между введенными ранее комодульными структурами и гомоморфизмами. 
УтВЕРЖДЕНИЕ 9.11. Следующая диаграмма коммутативна:

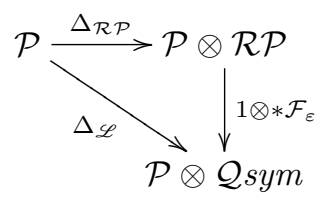

Доказательство. Пусть $P-n$-мерный многогранник. Тогда

$$
\begin{aligned}
& \Delta_{\mathscr{L}}(P)=\sum_{k=0}^{n} \sum_{\left(j_{1}, \ldots, j_{k}\right)}\left(d_{j_{1}} \cdots d_{j_{k}} P\right) \otimes M_{\left(j_{1}, \ldots, j_{k}\right)} \\
& =\sum_{k=0}^{n} \sum_{\left(j_{1}, \ldots, j_{k}\right)} \sum_{F^{n-\left(j_{1}+\cdots+j_{k}\right)}}\left(F^{n-\left(j_{1}+\cdots+j_{k}\right)} \sum_{\substack{F^{n-\left(j_{1}+\cdots+j_{k}\right)} \subset \\
\subset F^{n-\left(j_{2}+\cdots+j_{k}\right)} \subset \\
\subset \cdots \subset F^{n-j_{k}} \subset P}} 1\right) \otimes M_{\left(j_{1}, \ldots, j_{k}\right)} \\
& =\sum_{r=0}^{n} \sum_{F^{r} \subseteq P} F^{r} \otimes\left(\sum_{k=0}^{n-r} \sum_{\begin{array}{c}
\left(j_{1}, \ldots, j_{k}\right): \\
j_{1}+\cdots+j_{k}=n-r
\end{array}} \sum_{\substack{F^{r} \subset F^{r+j_{1}} \subset \\
\subset \cdots \subset F^{n-j_{k}} \subset P}} M_{\left(j_{1}, \ldots, j_{k}\right)}\right) \\
& =\sum_{G \subseteq P} G \otimes * \mathcal{F}_{\varepsilon}(P / G)=\left(1 \otimes * \mathcal{F}_{\varepsilon}\right) \Delta_{\mathcal{R} \mathcal{P}}(P) .
\end{aligned}
$$

СлЕДСТВИЕ 9.12. Для любого элемента $P \in \mathcal{P}$ выполнено равенство $\mathcal{F}_{\varepsilon}\left(l_{\alpha} P\right)=\mathcal{F}_{\mathcal{P}}(P)$, m. e. $\mathcal{F}_{\mathcal{P}}=\xi_{\alpha} \star \mathcal{F}_{\varepsilon}$.

ДокАЗАТЕЛЬСтво. Имеем: $\left(\xi_{\alpha} \otimes 1\right) \Delta_{\mathscr{L}} P=\mathcal{F}_{\mathcal{P}}(P)^{*}$. Если теперь применить гомоморфизм $\xi_{\alpha} \otimes 1$ к диаграмме $(75)$, то получим $* \mathcal{F}_{\varepsilon} l_{\alpha}=\left(\xi_{\alpha} \otimes 1\right) \Delta_{\mathscr{L}}=* \mathcal{F}_{\mathcal{P}}$. Следовательно, $\mathcal{F}_{\varepsilon} l_{\alpha}=\mathcal{F}_{\mathcal{P}}$.

СлЕДСтвие 9.13. Следующая диаграмма коммутативна:

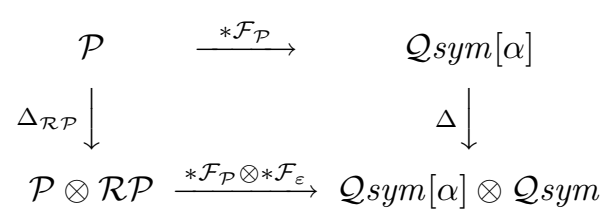

ДокАЗАтельство. Рассмотрим коммутативную диаграмму:

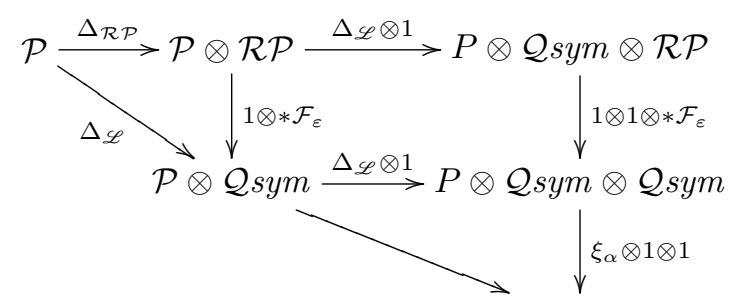

$\mathbb{Z}[\alpha] \otimes \mathcal{Q}$ sym $\otimes \mathcal{Q}$ sym

Тогда

$\left(\xi_{\alpha} \otimes 1 \otimes 1\right)\left(\Delta_{\mathscr{L}} \otimes 1\right) \Delta_{\mathscr{L}}=\left(\xi_{\alpha} \otimes 1 \otimes 1\right)(1 \otimes \Delta) \Delta_{\mathscr{L}}=\Delta\left(* \mathcal{F}_{\mathcal{P}}\right)$, 
в то время как

$$
\left(\xi_{\alpha} \otimes 1 \otimes 1\right)\left(1 \otimes 1 \otimes * \mathcal{F}_{\varepsilon}\right)\left(\Delta_{\mathscr{L}} \otimes 1\right) \Delta_{\mathcal{R} \mathcal{P}}=\left(* \mathcal{F}_{\mathcal{P}} \otimes * \mathcal{F}_{\varepsilon}\right) \Delta_{\mathcal{R P}} .
$$

Так как эти две композиции отображений соответствуют двум обходам диаграммы, то они равны.

\section{0. Универсальный $G$-полином}

Пусть $\mathbb{A}$ - коммутативное ассоциативное кольцо с единицей и $A=\sum A^{2 n}$, $n \geqslant 0,-$ связная градуированная ассоциативная $\mathbb{A}$-алгебра. Рассмотрим градуированное кольцо $A[\alpha, t], \operatorname{deg} \alpha=\operatorname{deg} t=2$.

Приведем конструкцию кольцевых гомоморфизмов $G: A \rightarrow \mathbb{A}[\alpha, t]$. При $A=\mathcal{P}_{s}$ в частном случае мы получим гомоморфизм $P^{n} \rightarrow f_{0, n} t^{n}$, а при $A=\mathcal{R} \mathcal{P}$ в частном случае - торические $g$ - и $h$-полиномы, введенные в [37].

10.1. Конструкция. В ряде направлений исследования важную роль играет понятие деформации умножения (см. [19] и [30]). Здесь мы получим результаты, использующие деформацию умножения в задаче о флаговых числах выпуклых многогранников.

ОПРЕДЕЛЕНИЕ 10.1 (деформация умножения). Градуированной деформацией умножения в $\mathbb{A}$-алгебре $A$ называется гомоморфизм градуированных $\mathbb{A}$-алгебр $\Psi: A \rightarrow A[\alpha, t], \Psi(a)=\Psi(a ; \alpha, t)$, такой, что $\Psi(a ; 0,0) \equiv a, a \in A$.

Пусть $\tau: \mathbb{A}[\alpha, t] \rightarrow \mathbb{A}[\alpha, t]-$ кольцевой гомоморфизм, меняющий местами $\alpha$ и $t$. Доказательство следующего результата использует рассуждения из [38].

Теорема 10.2 (конструкция $G$-полинома). Пусть $\Psi: A \rightarrow A[\alpha, t]-$ градуированная деформачия умножения в $\mathbb{A}$-алгебре $A$. Тогда

1) существует единственная пара градуированных $\mathbb{A}$-линейных отображениц $\widetilde{G}=\widetilde{G}_{\Psi}$ и $G=G_{\Psi}: A \rightarrow \mathbb{A}[\alpha, t], G(1)=\widetilde{G}(1)=1$, таких, umo

(a) $G(a)=\sum g_{i} \alpha^{i} t^{n+1-i}, 0 \leqslant i \leqslant[n / 2], g_{i} \in \mathbb{A}$, для любого $a \in A^{2(n+1)}$,

(b) $\widetilde{G}(a)=\sum_{a \in A^{2(n+1)}} \widetilde{g}_{i} \alpha^{i} t^{n+1-i},[n / 2]+1 \leqslant i \leqslant n+1, \widetilde{g}_{i} \in \mathbb{A}$, для любого

(с) отображения $G$ и $\widetilde{G}$ связаны уравнением $\widetilde{G}=G \Psi$;

2) отображения $\widetilde{G}$ и $G$ являются гомоморфизмами $\mathbb{A}$-алгебр;

3) если в кольце $A$ нет 2 -кручения $и \Psi(\Psi(a ; t, \alpha) ; \alpha, t)=$ а для любого $a \in A$, mo $\widetilde{G}=\tau G$.

ДокАЗАТЕЛЬСтво. Применим индукцию по $n$. При $a \in \mathbb{A}$ утверждение 1$)$ очевидно. Пусть отображения $G$ и $\widetilde{G}$ определены на всех группах $A^{2 k}, k \leqslant n$, и являются $\mathbb{A}$-линейными. Возьмем $a \in A^{2(n+1)}$, тогда имеет место уравнение

$$
\widetilde{G}(a)-G(a)=G(\Psi(a)-a) .
$$

Полином $\Psi(a)-a \in A[\alpha, t]$ является однородным степени $2(n+1)$, причем все коэффициенты при мономах $\alpha^{i} t^{j}$ являются однородными элементами кольца $A$ меньшей степени. Поэтому полином в правой части уравнения (76) однозначно определен и является однородным степени $2(n+1)$. Так как множества 
мономов $\alpha^{i} t^{j}$ с ненулевыми коэффициентами в $G(a)$ и $\widetilde{G}(a)$ не пересекаются, то эти полиномы определяются из уравнения (76) единственным образом. Так как $\Psi-\mathbb{A}$-линейное отображение, то отображения $G, \widetilde{G}: A^{2(n+1)} \rightarrow \mathbb{A}[\alpha, t]$ являются $\mathbb{A}$-линейными.

Так как $\widetilde{G}=G \Psi$ и $\Psi$ - кольцевой гомоморфизм, то для доказательства утверждения 2) достаточно показать, что $G$ - кольцевой гомоморфизм. Для этого применим индукцию. Так как $G(a \cdot 1)=G(a) G(1)$ и $G(1 \cdot a)=G(1) G(a)$, то $G(a b)=G(a) G(b)$, если $a \in \mathbb{A}$ или $b \in \mathbb{A}$. Предположим, что эта формула верна для любых элементов $a \in A^{2 p}, b \in A^{2 q}$, таких, что $p \leqslant n_{1}+1, q \leqslant n_{2}+1$, где хотя бы одно из неравенств строгое. Пусть $x \in A^{2\left(n_{1}+1\right)}$ и $y \in A^{2\left(n_{2}+1\right)}$, тогда

$$
\begin{aligned}
\widetilde{G}(x y)-G(x y) & =G[\Psi(x y)-x y]=G[\Psi(x) \Psi(y)-x y] \\
& =G[(\Psi(x)-x) \Psi(y)+x(\Psi(y)-y)] .
\end{aligned}
$$

Каждый из коэффициентов при мономах $\alpha^{i} t^{j}$, входящих в полиномы $\Psi(x)-x$ и $\Psi(y)-y$, имеет степень меньшую, чем $n_{1}$ и $n_{2}$ соответственно, поэтому получаем:

$$
\begin{aligned}
\widetilde{G}(x y) & -G(x y)=(G[\Psi(x)-x]) G[\Psi(y)]+G(x)(G[\Psi(y)-y]) \\
= & (\widetilde{G}(x)-G(x)) \widetilde{G}(y)+G(x)(\widetilde{G}(y)-G(y))=\widetilde{G}(x) \widetilde{G}(y)-G(x) G(y) .
\end{aligned}
$$

По $\alpha$ однородные полиномы $G(x y), G(x)$ и $G(y)$ имеют степени, не превосходящие $\left[\left(n_{1}+n_{2}+1\right) / 2\right],\left[n_{1} / 2\right]$ и $\left[n_{2} / 2\right]$ соответственно. Каждый моном с ненулевым коэффициентом в полиномах $\widetilde{G}(x y)$ и $\widetilde{G}(x) \widetilde{G}(y)$ имеет по $\alpha$ степень не менее $\left[\left(n_{1}+n_{2}+1\right) / 2\right]+1$ и $\left[n_{1} / 2\right]+\left[n_{2} / 2\right]+2$ соответственно. Имеем $\left[\left(n_{1}+n_{2}+1\right) / 2\right]+1>\left[\left(n_{1}+n_{2}+1\right) / 2\right] \geqslant\left[n_{1} / 2\right]+\left[n_{2} / 2\right]$ и $\left[n_{1} / 2\right]+\left[n_{2} / 2\right]+2>$ $\left[\left(n_{1}+n_{2}+1\right) / 2\right] \geqslant\left[n_{1} / 2\right]+\left[n_{2} / 2\right]$, поэтому $G(x y)=G(x) G(y)$.

Третье утверждение теоремы также докажем по индукции. Для $a \in \mathbb{A}$ утверждение верно, так как $\widetilde{G}(a)=a=G(a)$. Пусть утверждение верно для элементов групп $A^{2 k}, k \leqslant n$, и $a \in A^{2(n+1)}$. Тогда

$$
\begin{aligned}
\tau[G(\Psi(a)-a)] & =(\tau G)(\Psi(a ; t, \alpha)-a)=\widetilde{G}(\Psi(a ; t, \alpha)-a) \\
& =G \Psi(\Psi(a ; t, \alpha)-a ; \alpha, t)=G(a-\Psi(a))=-G(\Psi(a)-a),
\end{aligned}
$$

поэтому в уравнении (76) правая часть является кососимметрической по переменным $\alpha$ и $t$. Тогда $\tau \widetilde{G}(a)+\widetilde{G}(a)=\tau G(a)+G(a)$.

При $n=2 k$ имеем: $[n / 2]=k,[n / 2]+1=k+1=n+1-[n / 2]$, поэтому $\widetilde{g}_{i}=g_{n+1-i},[n / 2]+1 \leqslant i \leqslant n+1$; а при $n=2 k+1$ имеем $[n / 2]=k,[n / 2]+1=$ $k+1=n+1-([n / 2]+1)$ и $k+2=n+1-[n / 2]$, поэтому $2 \widetilde{g}_{[n / 2]+1}=0$ и $\widetilde{g}_{i}=g_{n+1-i}$ при $[n / 2]+2 \leqslant i \leqslant n+1$. В обоих случаях получаем: $\widetilde{G}(a)=\tau G(a)$.

СлеДСтвиЕ 10.3. Пусть $\Psi(a)=а$ для любого $a \in A$. Тогда $G=\widetilde{G}=\varepsilon_{A}$ отображение аугментации. Поэтому для произвольной градуированной деформации $\Psi$ умножения в кольце $A$ индуцированные гомоморфизмы $G_{\Psi}$ и $\widetilde{G}_{\Psi}$ являются "дебормациями" аугментации.

ПримеР 10.4. Пусть $A=\mathbb{Z}[x], \operatorname{deg} x=2$. Любая градуированная деформация умножения $\Psi(\alpha, t): \mathbb{Z}[x] \rightarrow \mathbb{Z}[x, \alpha, t]$ переводит $x$ в $x+p \alpha+q t, p, q \in \mathbb{Z}$, поэтому $\Psi(a ; \alpha, t)=a(x+p \alpha+q t)$. Тогда $\widetilde{G}(x)-G(x)=\Psi(x)-x=p \alpha+q t$, 
поэтому $G(x)=-q t$ и $\widetilde{G}(x)=p \alpha$. При этом $\widetilde{G}=\tau G$ тогда и только тогда, когда $p=-q$, что равносильно тому, что $\Psi(\Psi(x ; t, \alpha) ; \alpha, t)=x$.

Пример 10.5. Пусть $A=\mathbb{Z}\left[x_{1}, x_{2}, \ldots\right], \operatorname{deg} x_{i}=2 i$. Зададим дифференцирование $d: A \rightarrow A$ и градуированную деформацию $\Psi: A \rightarrow A[\alpha, t]$ формулами

$$
d x_{i}=(i+1) x_{i-1}, \quad x_{0}=1, \quad d x_{0}=0, \quad \Psi(x)=\exp ((\alpha-t) d) x .
$$

Тогда $G\left(x_{i}\right)=(i+1) t^{i}=\varepsilon_{A}\left(\exp (t d) x_{i}\right)$ и $\widetilde{G}\left(x_{i}\right)=(i+1) \alpha^{i}=\varepsilon_{A}\left(\exp (\alpha d) x_{i}\right)$. Действительно, имеем:

$\varepsilon_{A}(\exp (\alpha d) x)=\varepsilon_{A}(\exp (t d) \exp ((\alpha-t) d) x), \quad \varepsilon\left(\exp (t d) x_{0}\right)=1=\varepsilon\left(\exp (\alpha d) x_{0}\right)$, поэтому из единственности отображений $\widetilde{G}$ и $G$ следует, что $G=\varepsilon_{A} \exp (t d)$ и $\widetilde{G}=\varepsilon_{A} \exp (\alpha d)$.

УтВЕРЖДЕНИЕ 10.6 (функториальность). Пусть $A, B$ - градуированные $\mathbb{A}$-алгебры, $\Psi_{A}: A \rightarrow A[\alpha, t], \Psi_{B}: B \rightarrow B[\alpha, t]$ - градуированные деформации умножений и $\Lambda: A \rightarrow B$ - гомоморфизм градуированных $\mathbb{A}$-алгебр такой, что $\Lambda \Psi_{A}=\Psi_{B} \Lambda$. Тогда $G_{A}=G_{B} \Lambda u \widetilde{G}_{A}=\widetilde{G}_{B} \Lambda$.

ДоказАТЕЛЬСтво. Имеем $\widetilde{G}_{B} \Lambda=G_{B} \Psi_{B} \Lambda=G_{B} \Lambda \Psi_{A}$ и $G_{B}(\Lambda(1))=$ $G_{B}(1)=1=\widetilde{G}_{B}(1)=\widetilde{G}_{B}(\Lambda(1))$, поэтому из единственности пары отображений $\widetilde{G}$ и $G$ получаем: $G_{A}=G_{B} \Lambda$ и $\widetilde{G}_{A}=\widetilde{G}_{B} \Lambda$.

\section{2. Приложения.}

Конструкция 10.7. Пусть $\mathcal{H}$ - алгебра Лейбница-Хопфа. Рассмотрим ряд $\Phi(\alpha-t)=\sum H_{k}(\alpha-t)^{k}, \quad k \geqslant 0$. Имеем $\Delta \Phi=\Phi \otimes \Phi$, поэтому задана каноническая градуированная деформация умножения в градуированно двойственной алгебре $\mathcal{H}^{*}$ по формуле $\Psi(a ; \alpha, t)=\Phi(\alpha-t) a$, где $\left\langle\Phi(\alpha-t) a, H_{\omega}\right\rangle=$ $\left\langle a, H_{\omega} \Phi(\alpha-t)\right\rangle$.

ОПРЕДЕЛЕНИЕ 10.8. Отображение $G_{\mathcal{H}^{*}}: \mathcal{H}^{*} \rightarrow \mathbb{Z}[\alpha, t]$ будем называть $G$-noлиномом. Отображение $\mathcal{G}=G_{\mathcal{Z}^{*}}: \mathcal{Z}^{*}=\mathcal{Q} s y m \rightarrow \mathbb{Z}[\alpha, t]$ назовем универсальнъм $G$-полиномом.

ПРимеР 10.9. Отображение $\Phi(t)$ переводит квазисимметрический моном $M_{\left(j_{1}, \ldots, j_{k-1}, j_{k}\right)}$ в $M_{\left(j_{1}, \ldots, j_{k-1}, j_{k}\right)}+t^{j_{k}} M_{\left(j_{1}, \ldots, j_{k-1}\right)}$, поэтому

$$
\widetilde{\mathcal{G}}\left(M_{\left(j_{1}, \ldots, j_{k-1}, j_{k}\right)}\right)-\mathcal{G}\left(M_{\left(j_{1}, \ldots, j_{k-1}, j_{k}\right)}\right)=(\alpha-t)^{j_{k}} \mathcal{G}\left(M_{\left(j_{1}, \ldots, j_{k-1}\right)}\right) .
$$

В частности, $\mathcal{G}\left(M_{(n+1)}\right)=\sum_{i=0}^{[n / 2]}(-1)^{n-i}\left(\begin{array}{c}n+1 \\ i\end{array}\right) \alpha^{i} t^{n+1-i}$.

Для элементарных симметрических функций $\left\{\sigma_{i}=M_{(1, \ldots, 1)}, \sigma_{0}=1\right\}$ имеем: $\Phi(t) \sigma_{i}=\sigma_{i}+t \sigma_{i-1}, \widetilde{\mathcal{G}}\left(\sigma_{0}\right)=\mathcal{G}\left(\sigma_{0}\right)=1$ и $\widetilde{\mathcal{G}}\left(\sigma_{i}\right)-\mathcal{G}\left(\sigma_{i}\right)=(\alpha-t) \mathcal{G}\left(\sigma_{i-1}\right)$. Например, $\mathcal{G}\left(\sigma_{1}\right)=t, \mathcal{G}\left(\sigma_{2}\right)=t^{2}, \mathcal{G}\left(\sigma_{3}\right)=t^{3}-\alpha t^{2}, \mathcal{G}\left(\sigma_{4}\right)=t^{4}-2 \alpha t^{3}$.

Для любой алгебры Лейбница-Хопфа $\mathcal{H}$ классифицирующее отображение $\pi_{\mathcal{H}}: \mathcal{Z} \rightarrow \mathcal{H}, \pi_{\mathcal{H}}\left(Z_{i}\right)=H_{i}$, является эпиморфизмом, поэтому двойственное отображение $\pi_{\mathcal{H}}^{*}$ задает вложение $\mathcal{H}^{*} \rightarrow \mathcal{Q}$ sym.

Доказательства следующих результатов непосредственно вытекают из свойства функториальности. 
СлЕДСТвиЕ 10.10. Имеем: $G_{\mathcal{H}^{*}}=\mathcal{G} \pi_{\mathcal{H}}^{*}$.

СлеДСтвиЕ 10.11. Для деформации $* \Phi(\alpha-t) *$ умножения в кольце $\mathcal{Q} s y m$, где $\left\langle(* \Phi(\alpha-t) *) \psi, Z_{\omega}\right\rangle=\left\langle\psi, \Phi(\alpha-t) Z_{\omega}\right\rangle$, имеем: $G_{* \Phi *}=\mathcal{G} *, \widetilde{G}_{* \Phi *}=\widetilde{\mathcal{G}} *$.

Конструкция 10.12. Пусть $X=\sum X^{2 n}, n \geqslant 0,-$ градуированный модуль Милнора над универсальной алгеброй Лейбница-Хопфа $\mathcal{Z}$. Тогда соответствие $x \rightarrow \Psi(x ; \alpha, t)=\Phi(\alpha-t) x=\sum\left(Z_{n} x\right)(\alpha-t)^{n}, n \geqslant 0$, задает градуированную деформацию умножения в $X$. Назовем $G$-полиномом $G_{X}(x)$ элемента $x \in X$ полином $G_{\Psi}(x)$. Назовем $H$-полиномом $H_{X}(x)$ элемента $x \in X$ полином $\left(\widetilde{G}_{\Psi}(x)-G_{\Psi}(x)\right) /(\alpha-t)$.

Непосредственно из конструкции вытекает следующий результат.

УТВЕРЖДЕНИЕ 10.13. Пусть $\Phi(-t) \Phi(t) x=x$ для любого $x \in X$. Тогда $H$-полином любого элемента $x \in X$ является симметрическим.

ДоказАтельство. Имеем: $\Psi(\Psi(x ; t, \alpha) ; \alpha, t)=\Phi(\alpha-t) \Phi(t-\alpha) x$, следовательно, $\Psi(\Psi(x ; t, \alpha) ; \alpha, t)=x$ для любого $x \in X$ тогда и только тогда, когда $\Phi(-t) \Phi(t) x=x$ для любого $x \in X$. В этом случае $\widetilde{G}=\tau G_{X}$ и $H_{X}(x)=$ $\left(\tau G_{X}(x)-G_{X}(x)\right) /(\alpha-t)$, поэтому $H$-полином любого элемента $x \in X$ является симметрическим.

Формула $\left\langle\varphi_{X}(x), Z\right\rangle=\varepsilon(Z x)$ задает каноническое отображение $\varphi_{X}: X \rightarrow$ Qsym.

УТВЕРЖДЕНИЕ 10.14. $\varphi_{X} \Phi(\alpha-t)=\Phi(\alpha-t) \varphi_{X}$.

ДокАЗАТЕЛЬСтво. Действительно,

$$
\begin{aligned}
\left\langle\varphi_{X}(\Phi(\alpha-t) x), Z\right\rangle & =\varepsilon(Z \Phi(\alpha-t) x)=\left\langle\varphi_{X}(x), Z \Phi(\alpha-t)\right\rangle \\
& =\left\langle\Phi(\alpha-t) \varphi_{X}(x), Z\right\rangle .
\end{aligned}
$$

СлЕДСТвИЕ 10.15. $G_{X}=\mathcal{G} \varphi_{X}$.

ПримеР 10.16 (торические $g$ - и $h$-полиномы). Для алгебры Рота-Хопфа $\mathcal{R}$ получаем кольцевой гомоморфизм $G_{\mathcal{R}}: \mathcal{R} \rightarrow \mathbb{Z}[\alpha, t]$ и линейное отображение $H_{\mathcal{R}}: \mathcal{R} \rightarrow \mathbb{Z}[\alpha, t]$. Для частично упорядоченного множества $T$ ранга $n+1$ полиномы $G_{\mathcal{R}}(T)$ и $H_{\mathcal{R}}(T)$ однозначно определяются из рекуррентных соотношений:

1) если $n+1=0$, то $G_{\mathcal{R}}(T)=1, H_{\mathcal{R}}(T)=0$;

2) если $n+1>0$, то $H_{\mathcal{R}}(T)=\sum_{y \in T \backslash \hat{1}} G_{\mathcal{R}}([\hat{0}, y])(\alpha-t)^{n-\rho(y)}$;

3) если $n+1>0$ и $H_{\mathcal{R}}(T)=\sum \kappa_{i} \alpha^{i} t^{n-i}, 0 \leqslant i \leqslant n$, то

$$
G_{\mathcal{R}}(P)=\kappa_{0} t^{n+1}+\left(\kappa_{1}-\kappa_{0}\right) \alpha t^{n}+\cdots+\left(\kappa_{[n / 2]}-\kappa_{[n / 2]-1}\right) \alpha^{[n / 2]} t^{n+1-[n / 2]} .
$$

В этом случае полиномы $G_{\mathcal{R}}(x, 1)$ и $H_{\mathcal{R}}(x, 1)$ совпадают с торическими $g$ $u$-полиномами $g(T, x)$ и $h(T, x)$, введенными в [37] для случая эйлеровых градуированных частично упорядоченных множеств и использованными для произвольных градуированных частично упорядоченных множеств в работах [39], [38]. См. также [31], [40]-[46].

Наборы чисел $\left(g_{0}, \ldots, g_{[n / 2]}\right)$ и $\left(h_{0}, \ldots, h_{n}\right), h_{i}=\kappa_{n-i}$, называются торическими $g$ - $u$ h-векторами. 
Легко видеть, что отображение $\varphi_{\mathcal{R}}$ совпадает с отображением Эренборга F, поэтому мы получаем следующий результат.

УТВЕРЖДЕНИЕ 10.17. $G_{\mathcal{R}}=\mathcal{G F}$.

ЗАмечАнИЕ 10.18. В работе [37] полином $h(T, x)$ равен 1 , если $\rho(T)=0$. В нашей конструкции более естественно считать, что в этом случае $h(T, x)=0$.

ЗАмЕчАнИЕ 10.19. В работе [40] впервые было замечено, что торический $g$-полином является мультипликативным относительно джойна. В работе [38] построен кольцевой гомоморфизм $g: \mathcal{Q} s y m \rightarrow \mathbb{Z}[x]$ такой, что $g(\mathrm{~F}(T))=g(T, x)$. Для доказательства существования такого отображения и его мультипликативности используется то, что гомоморфизм $\mathrm{F}: \mathcal{R} \rightarrow \mathcal{Q}$ sym является эпиморфизмом. В настоящем обзоре предложена функториальная конструкция $G$-полинома, которая в случаях колец $\mathcal{Q} s y m$ и $\mathcal{R}$ дает соответственно отображения $\mathcal{G}$ и $G_{\mathcal{R}}$ такие, что $G_{\mathcal{R}}=\mathcal{G F}, \mathcal{G}\left(M_{\omega} ; x, 1\right)=g\left(M_{\omega}\right)$ и $G_{\mathcal{R}}(T ; x, 1)=g(T, x)$.

ПримеР 10.20 (торические $h$-векторы решеток граней).

\begin{tabular}{|c|c|}
\hline $\mathrm{pt}$ & $(1)$ \\
$I$ & $(1,1)$ \\
$M_{m}^{2}$ & $(1, m-2,1)$ \\
$P_{m}^{3}$ & $(1, m-3,1)$ \\
\hline
\end{tabular}

где $P_{m}^{3}$ - трехмерный многогранник с $m$ гипергранями.

Пример 10.21. Так как $\Phi(-t) \Phi(t)=1$ на кольце $\mathrm{R}$, то мы получаем кольцевой гомоморфизм $G_{\mathrm{R}}: \mathrm{R} \rightarrow \mathbb{Z}[\alpha, t]$ и линейное отображение $H_{\mathrm{R}}: \mathrm{R} \rightarrow \mathbb{Z}\left[\sigma_{1}, \sigma_{2}\right]$, где $\sigma_{1}=\alpha+t, \sigma_{2}=\alpha t$.

В случае кольца $\mathcal{R} \mathcal{P}$ имеем: $G_{\mathcal{R} \mathcal{P}}(P)=G_{\mathcal{R}}(L(P))$ и $H_{\mathcal{R} \mathcal{P}}(P)=H_{\mathcal{R}}(L(P))$. Отображение $\varphi_{\mathcal{R}}$ совпадает с $* \mathcal{F}_{\varepsilon}$, поэтому получаем следующий результат.

УТВЕРЖДЕНИЕ 10.22. $G_{\mathcal{R} \mathcal{P}}(P)=\mathcal{G}\left(* \mathcal{F}_{\varepsilon}(P)\right)$.

На кольце простых многогранников $\mathcal{P}_{s}$ имеем: $\Phi(\alpha-t)=\exp [(\alpha-t) d]$. Тогда

$$
\xi_{0} \exp [\alpha d]=\xi_{0} \exp [t d] \exp [(\alpha-t) d], \quad \xi_{0} \exp [t d] \mathrm{pt}=1,
$$

поэтому $G_{\mathcal{P}}\left(P^{n}\right)=\xi_{0} \exp [t d] P^{n}=f_{0, n}\left(P^{n}\right) t^{n}$ и $H_{\mathcal{P}}\left(P^{n}\right)=f_{0, n}\left(\alpha^{n}-t^{n}\right) /(\alpha-t)$ для любого простого $n$-мерного многогранника $P^{n}$.

УтВеРЖДЕНИЕ 10.23. Рассмотрим деформацию умножения $* \Phi(\alpha-t) *$ : $\mathcal{R P} \rightarrow \mathcal{R P}[\alpha, t]$. Тогда $(* \Phi(\alpha-t) *) P=\sum_{\varnothing \subseteq F \subseteq P} P / F u G_{\mathcal{R P}, * \Phi *}=G_{\mathcal{R} \mathcal{P}} *$

10.3. $g$ - и $h$-полиномы выпуклых многогранников. Как приложение универсальной конструкции $G$-полинома, введем $g$ - и $h$-полиномы выпуклых многогранников, обобщающие однородные $g$ - и $h$-полиномы простых многогранников (см. [2]), где

$$
\begin{gathered}
h(\alpha, t)\left(P^{n}\right)=\sum_{i=0}^{n} h_{i} t^{i} \alpha^{n-i}=\sum_{i=0}^{n} \kappa_{i} \alpha^{i} t^{n-i}=f(\alpha-t, t)\left(P^{n}\right), \\
g(\alpha, t)\left(P^{n}\right)=\kappa_{0} t^{n+1}+\left(\kappa_{0}-\kappa_{1}\right) \alpha t^{n}+\cdots+\left(\kappa_{[n / 2]}-\kappa_{[n / 2]-1}\right) \alpha^{[n / 2]} t^{n+1-[n / 2]} .
\end{gathered}
$$


ОПРЕДЕЛЕНИЕ 10.24 ( $g$ - и $h$-полиномы выпуклых многогранников). Мы называем $g$-полиномом выпуклого многогранника $P^{n}$ полином

$$
g(\alpha, t)(P)=G_{\mathcal{R} \mathcal{P}, * \Phi *}(P)=G_{\mathcal{R} \mathcal{P}}\left(P^{*}\right)=\sum g_{i} \alpha^{i} t^{n+1-i}, \quad 0 \leqslant i \leqslant\left[\frac{n}{2}\right] .
$$

$h$-полиномом выпуклого многогранника $P^{n}$ будем называть полином

$$
h(\alpha, t)(P)=\frac{g(t, \alpha)(P)-g(\alpha, t)(P)}{\alpha-t}=\sum h_{n-i} \alpha^{i} t^{n-i}, \quad 0 \leqslant i \leqslant n .
$$

Имеем:

$$
\begin{aligned}
& g(t, \alpha)(P)=\sum_{\varnothing \subseteq F \subseteq P}(\alpha-t)^{\operatorname{dim} F+1} g(\alpha, t)(P / F), \\
& h(\alpha, t)(P)=\sum_{F \subseteq P}(\alpha-t)^{\operatorname{dim} F} g(\alpha, t)(P / F) .
\end{aligned}
$$

Наборы чисел $\left(h_{0}, \ldots, h_{n}\right)$ и $\left(g_{0}, \ldots, g_{[n / 2]}\right)$ мы называем $h$ - $u g$-векторами многогранника $P^{n}$.

УТВЕРЖДЕНИЕ 10.25. $g(\alpha, t)(P)=\mathcal{G}\left[\mathcal{F}_{\varepsilon}(P)\right]$.

УтВЕРЖДЕНИЕ 10.26. $h(\alpha, t)(P)=\mathcal{G}\left[\mathcal{F}_{\mathcal{P}}\left(\alpha-t, t_{1}, t_{2}, \ldots\right)(P)\right]$.

ДоказАтельство. Согласно следствию 9.12 имеем: $\mathcal{F}_{\mathcal{P}}=\xi_{\alpha} \star \mathcal{F}_{\varepsilon}$, поэтому

$$
\mathcal{G}\left[\mathcal{F}_{\mathcal{P}}\left(\alpha-t, t_{1}, t_{2}, \ldots\right)(P)\right]=\mathcal{G}\left[\xi_{\alpha-t} \star \mathcal{F}_{\varepsilon}(P)\right]=\left(\xi_{\alpha-t} \star g\right)(P)=h(\alpha, t)(P) .
$$

$\mathrm{B}$ частности получаем известный факт, что коэффициенты $g$ - и $h$-полиномов являются целочисленными комбинациями флаговых чисел.

ЗАмечание 10.27. Мы имеем $g(\alpha, t)(P)=G_{\mathcal{R} \mathcal{P}}\left(P^{*}\right)$ и $h(\alpha, t)(P)=H_{\mathcal{R P}}\left(P^{*}\right)$. Это связано с тем, что торические $g$ - и $h$-полиномы обобщают $g$ - и $h$-полиномы симплициальных многогранников, а $g$ - и $h$-полиномы в настоящем обзоре обобщают соответствующие полиномы простых многогранников.

ПримеР 10.28. Для точки $x=\mathrm{pt}$ имеем

$$
\tau G_{\mathcal{R P}}(x)-G_{\mathcal{R P}}(x)=G_{\mathcal{R P}}(\Phi(\alpha-t) x-x)=G_{\mathcal{R} \mathcal{P}}((\alpha-t) \varnothing)=(\alpha-t),
$$

поэтому $g(\alpha, t)(x)=G_{\mathcal{R} \mathcal{P}}\left(x^{*}\right)=G_{\mathcal{R} \mathcal{P}}(x)=t$. Так как $\Delta^{n}=x^{n+1}$, то получаем: $g(\alpha, t)\left(\Delta^{n}\right)=t^{n+1}$.

Teорема 10.29. 1) Соответствие $P \rightarrow h(\alpha, t)(P)$ задает гомоморфизм градуированных колеи, $\mathcal{P} \rightarrow \mathbb{Z}[\alpha, t]$ такой, что $h=\xi_{\alpha-t} \star g$.

2) $h(t, \alpha)(P)=h(P)(\alpha, t)$ для любого $P \in \mathcal{P}$.

3) Если $P$ - простой многогранник, то $h(\alpha, t)(P)=f(\alpha-t, t)(P)$.

ДокАЗАТЕЛЬСтво. Из конструкции видно, что $h=\xi_{\alpha-t} \star g$. Поскольку отображение $g$ является гомоморфизмом градуированных колец, то и $h$ является гомоморфизмом градуированных колец.

Так как $h(\alpha, t)(P)=(g(t, \alpha)(P)-g(\alpha, t)(P)) /(\alpha-t)$, то $h$-полином является симметрическим.

Если многогранник $P$ простой, то $P / P=\varnothing$ и для каждой его собственной непустой грани $F$ гранная фигура $P / F$ является симплексом размерности $\operatorname{dim} P-\operatorname{dim} F-1$, поэтому $g(\alpha, t)(P / F)=t^{\operatorname{dim} P-\operatorname{dim} F}$. Тогда $h(P)=\sum_{F \subseteq P}(\alpha-t)^{\operatorname{dim} F} g(\alpha, t)(P / F)=\sum_{F \subseteq P}(\alpha-t)^{\operatorname{dim} F} t^{\operatorname{dim} P-\operatorname{dim} F}=f(\alpha-t, t)(P)$. 
10.4. Комментарии. Одним из выдающихся результатов теории многогранников является так называемая $g$-теорема, сформулированная в 1970 г. в виде гипотезы П. Макмюлленом [47] и доказанная в 1980 г. Р. Стенли [48] (необходимость) и Л. Биллера и К. Ли [49] (достаточность).

Для любых натуральных чисел $a$ и $i$ существует и единственно "биномиальное $i$-разложение" числа $a$, а именно: $a=\left(\begin{array}{c}a_{i} \\ i\end{array}\right)+\left(\begin{array}{c}a_{i-1} \\ i-1\end{array}\right)+\cdots+\left(\begin{array}{c}a_{j} \\ j\end{array}\right)$, где $a_{i}>$ $a_{i-1}>\cdots>a_{j} \geqslant j \geqslant 1$. Положим $a^{\langle i\rangle}=\left(\begin{array}{c}a_{i}+1 \\ i+1\end{array}\right)+\left(\begin{array}{c}a_{i-1}+1 \\ i\end{array}\right)+\cdots+\left(\begin{array}{c}a_{j}+1 \\ j+1\end{array}\right)$, $0^{\langle i\rangle}=0$. Например, $a=\left(\begin{array}{l}a \\ 1\end{array}\right)$, поэтому $a^{\langle 1\rangle}=\left(\begin{array}{c}a+1 \\ 2\end{array}\right)=\frac{(a+1) a}{2} ;$ для $i \geqslant a$ мы имеем: $a=\left(\begin{array}{l}i \\ i\end{array}\right)+\left(\begin{array}{l}i-1 \\ i-1\end{array}\right)+\cdots+\left(\begin{array}{c}i-a+1 \\ i-a+1\end{array}\right)$, поэтому $a^{\langle i\rangle}=a$.

Теорема 10.30 ( $g$-теорема, [48], [49]). Набор чельх чисел $\left(g_{0}, g_{1}, \ldots, g_{[n / 2]}\right)$ образует g-вектор простого п-мерного многогранника тогда и только тогда, когда он удовлетворяет следуюшим условиям: $g_{0}=1,0 \leqslant g_{1}, 0 \leqslant g_{i+1} \leqslant g_{i}^{\langle i\rangle}$, $i=1,2, \ldots,[n / 2]-1$.

В работе [48] Р. Стенли для простого многогранника $P$ построил торическое многообразие $X_{P}$ такое, что $\left\{h_{i}\right\}$ - четные числа Бетти кольца (сингулярных) когомологий многообразия $X_{P}$. Тогда условия Макмюллена следуют из двойственности Пуанкаре и сильной теоремы Лефшеца для многообразия $X_{P}$.

В работе [50] П. Макмюллен предложил чисто геометрическое доказательство этих условий, используя понятие алгебры многогранников.

В работе [37] Р. Стенли ввел понятие торического $h$-вектора произвольного выпуклого многогранника. При этом для многогранника с рациональными вершинами торические $h$-числа $\left\{h_{i}\right\}$ равны числам Бетти когомологий пересечений связанного с $P$ торического многообразия. (Доказательство см. в [51].)

Торический $h$-вектор неотрицателен: $h_{i} \geqslant 0$, симметричен: $h_{i}=h_{n-i}$, и в случае многогранника с рациональными вершинами сильная теорема Лефшеца для когомологий пересечений связанного с многогранником торического многообразия доказывает унимодальность: $h_{0} \leqslant h_{1} \leqslant \cdots \leqslant h_{[n / 2]}$.

Обобщив геометрические методы, предложенные Макмюлленом [50], K. Кару [52] доказал унимодальность торического $h$-вектора произвольного выпуклого многогранника.

Компоненты торического $h$-вектора являются целочисленными линейными комбинациями флаговых чисел (см., например, утверждение 10.26). Но, вообще говоря, он не несет в себе всю информацию о флаговом векторе. В работе [45] Ли предложил "расширенный торический” $h$-вектор, содержащий в себе всю информацию о флаговом векторе и состоящий из набора неотрицательных симметричных унимодальных векторов. Дж. Файн (см. [43], [44]) ввел обобщенные $h$ - и $g$-векторы, содержащие как подмножества торические $h$ - и $g$-векторы соответственно, однако не обязательно имеющие неотрицательные коэффициенты. 


\section{1. Гомоморфизмы колец выпуклых многогранников}

Комодульное отображение $\Delta_{\mathcal{R}}: \mathcal{P} \rightarrow \mathcal{P} \otimes \mathcal{R} \mathcal{P}$ и коумножение $\Delta: \mathcal{R} \mathcal{P} \rightarrow$ $\mathcal{R} \mathcal{P} \otimes \mathcal{R} \mathcal{P}$ дают универсальный способ построения мультипликативных гомоморфизмов колец $\mathcal{P}$ и $\mathcal{R} \mathcal{P}$ в виде сверток.

ПримеР 11.1. При помощи свертки $\eta \star \zeta=(\eta \otimes \zeta) \Delta_{\mathcal{R}}: \mathcal{P} \rightarrow R_{1} \otimes R_{2}$ гомоморфизмов $\eta: \mathcal{P} \rightarrow R_{1}$ и $\zeta: \mathcal{R P} \rightarrow R_{2}$ получаются следующие кольцевые гомоморфизмы.

1) id $\star \varepsilon_{t}=\Phi(t): \mathcal{P} \rightarrow \mathcal{P}[t]$.

2) $\xi_{\alpha} \star \mathrm{id}=l_{\alpha}: \mathcal{P} \rightarrow \mathcal{R} \mathcal{P}[\alpha]$.

3) $\xi_{\alpha} \star \varepsilon_{t}=f(\alpha, t): \mathcal{P} \rightarrow \mathbb{Z}[\alpha, t]$.

4) $\xi_{\alpha} \star \mathcal{F}_{\varepsilon}=\mathcal{F}_{\mathcal{P}}: \mathcal{P} \rightarrow \mathcal{Q} \operatorname{sym}[\alpha]$.

5) $\xi_{\alpha-t} \star g=h: \mathcal{P} \rightarrow \mathbb{Z}[\alpha, t]$.

6) Рассмотрим гомоморфизм $M_{\beta}=\varepsilon_{1, \beta, 1}: \mathcal{R P} \rightarrow \mathbb{Z}[\beta]$. Тогда кольцевой гомоморфизм $\tilde{f}=\xi_{\alpha} \star M_{\beta}: \mathcal{P} \rightarrow \mathbb{Z}[\alpha, \beta]$ имеет вид

$$
\tilde{f}(P)=\sum_{F \subseteq P} \alpha^{\operatorname{dim} F} \beta^{m(P / F)} .
$$

Этот гомоморфизм на подкольце простых многогранников совпадает с $f$-полиномом $f(\alpha, \beta)$, но в общем случае не определяется флаговыми векторами многогранников. Например, для трехмерного многогранника $P^{3}$ мы имеем:

$\tilde{f}\left(P^{3}\right)=\alpha^{3}+\sum_{F \in P} \alpha^{2} \beta+\sum_{E \in P} \alpha \beta^{2}+\sum_{v \in P} \beta^{\text {ind } v}=\alpha^{3}+f_{\{2\}} \alpha^{2} \beta+f_{\{1\}} \alpha \beta^{2}+\sum_{v \in P} \beta^{\text {ind } v}$,

где через $v, E, F$ обозначены соответственно вершины, ребра и грани многогранника $P^{3}$, a ind $v$ - количество ребер, содержащих вершину $v$.

7) Наиболее общий мультипликативный гомоморфизм, который можно построить при помощи градуировок колец $\mathcal{P}$ и $\mathcal{R} \mathcal{P}$, имеет вид $\xi_{\alpha, s} \star \varepsilon_{t, \beta, \gamma}: \mathcal{P} \rightarrow$ $\mathbb{Z}[\alpha, \beta, \gamma, t, s]:$

$$
\xi_{\alpha, s} \star \varepsilon_{t, \beta, \gamma}\left(P^{n}\right)=\sum_{F \subseteq P} \alpha^{\operatorname{dim} F} s^{m(F)} t^{\operatorname{dim} P-\operatorname{dim} F} \beta^{m(P / F)} \gamma^{f_{0, \operatorname{dim}(P / F)}(P / F)} .
$$

Можно рассматривать итерированную свертку кольцевых гомоморфизмов $\eta: \mathcal{P} \rightarrow R_{0}$ и $\zeta_{1}: \mathcal{R P} \rightarrow R_{1}, \ldots, \zeta_{n}: \mathcal{R} \mathcal{P} \rightarrow R_{n}:$

$$
\eta \star \zeta_{1} \star \cdots \star \zeta_{n}=\left(\eta \star \zeta_{1} \star \cdots \star \zeta_{n-1}\right) \star \zeta_{n}: \mathcal{P} \rightarrow R_{0} \otimes R_{1} \otimes \cdots \otimes R_{n},
$$

которая тоже является кольцевым гомоморфизмом. Например,

$$
\xi_{\alpha} \star \varepsilon_{t_{n}} \star \cdots \star \varepsilon_{t_{1}}=\mathcal{F}_{\mathcal{P}, n} .
$$

В некоторых случаях можно перейти к свертке бесконечного числа гомоморфизмов, например,

$$
\xi_{\alpha} \star \cdots \star \varepsilon_{t_{n}} \star \cdots \star \varepsilon_{t_{1}}=\mathcal{F}_{\mathcal{P}} .
$$

В случае коумножения $\Delta: \mathcal{R} \mathcal{P} \rightarrow \mathcal{R} \mathcal{P} \otimes \mathcal{R} \mathcal{P}$ определена классическая свертка кольцевых гомоморфизмов $\zeta_{1}: \mathcal{R P} \rightarrow R_{1}$ и $\zeta_{2}: \mathcal{R P} \rightarrow R_{2}$ :

$$
\zeta_{1} \star \zeta_{2}=\left(\zeta_{1} \otimes \zeta_{2}\right) \Delta: \mathcal{R P} \rightarrow R_{1} \otimes R_{2} .
$$

ПримеР 11.2. Рассмотрим некоторые примеры.

1) $\varepsilon_{\alpha} \star \varepsilon_{t_{n}} \star \cdots \star \varepsilon_{t_{1}}=\mathcal{F}_{\mathcal{R} \mathcal{P}, n} ; \quad \varepsilon_{\alpha} \star\left(\cdots \star \varepsilon_{t_{n}} \star \cdots \star \varepsilon_{t_{1}}\right)=\mathcal{F}_{\mathcal{R} \mathcal{P}}$. 
2) $\varepsilon \star \varepsilon_{t_{n}} \star \cdots \star \varepsilon_{t_{1}}=\mathcal{F}_{\varepsilon, n} ; \quad \varepsilon \star\left(\cdots \star \varepsilon_{t_{n}} \star \cdots \star \varepsilon_{t_{1}}\right)=\mathcal{F}_{\varepsilon}$.

3) $\tau g=\varepsilon_{\alpha-t} \star g$.

Используя формулу $\left(\Delta_{\mathcal{R}} \otimes 1\right) \Delta_{\mathcal{R}}=(1 \otimes \Delta) \Delta_{\mathcal{R}}$, получаем следующее утверждение.

УТВЕРЖДЕНИЕ 11.3. Для любых трех гомоморфизмов $\eta: \mathcal{P} \rightarrow R_{0} u \zeta_{1}$ : $\mathcal{R P} \rightarrow R_{1}, \zeta_{2}: \mathcal{R P} \rightarrow R_{2}$ имеет место формула ассоциативности:

$$
\left(\eta \star \zeta_{1}\right) \star \zeta_{2}=\eta \star\left(\zeta_{1} \star \zeta_{2}\right)
$$

\section{2. Проблема флаговых векторов}

Мы отмечали, что для любого простого многогранника $P^{n}$

$$
\mathcal{F}_{\mathcal{P}}\left(\alpha, t_{1}, t_{2}, \ldots\right)\left(P^{n}\right)=\mathcal{F}_{\mathcal{P}, 1}\left(\alpha, t_{1}+t_{2}+\cdots\right)\left(P^{n}\right)=\mathcal{F}_{\mathcal{P}, 1}\left(\alpha, \sigma_{1}\right)\left(P^{n}\right) .
$$

Единственное линейное соотношение на полином $\mathcal{F}_{\mathcal{P}, 1}$ простых многогранников, эквивалентное соотношениям Дена-Соммервилля для чисел граней, имеет вид $\mathcal{F}_{\mathcal{P}, 1}(-\alpha, t+\alpha)=\mathcal{F}_{\mathcal{P}, 1}(\alpha, t)$. Можно показать, что это условие является необходимым и достаточным для того, чтобы полином $p\left(\alpha, \sigma_{1}\right) \in \mathcal{Q} \operatorname{sym}[\alpha]$ был образом целочисленной комбинации простых многогранников.

$g$-теорема полностью описывает $f$-векторы простых многогранников, тем самым давая необходимое и достаточное условие того, что данный полином $p\left(\alpha, \sigma_{1}\right) \in \mathcal{Q} \operatorname{sym}[\alpha]$ является образом простого многогранника.

Как отмечается в работе [53], даже для четырехмерных выпуклых многогранников соответствующая проблема описания флаговых векторов является чрезвычайно сложной.

Современные подходы к получению линейных и нелинейных соотношений на флаговые числа основываются на понятиях $\boldsymbol{c d}$-индекса, торического $h$-вектора и его обобщений, кольца флаговых чисел (см. приложения ниже).

Посмотрим на проблему описания флаговых векторов выпуклых многогранников с точки зрения $\times$-кольца $\mathcal{P}$.

$g$-теорема описывает все полиномы $p\left(\alpha, t_{1}, t_{2}, \ldots\right) \in \mathcal{Q} s y m[\alpha]$, которые являются образами простых многогранников при отображении $\mathcal{F}_{\mathcal{P}}$. Мы получили критерий того, что полином $p\left(\alpha, t_{1}, t_{2}, \ldots\right) \in \mathcal{Q} \operatorname{sym}[\alpha]$ является целочисленной комбинацией образов выпуклых многогранников.

ЗАДАчА 12.1. Найти необходимое и достаточное условие того, что полином $p\left(\alpha, t_{1}, t_{2}, \ldots\right) \in \mathcal{Q} \operatorname{sym}[\alpha]$ является образом выпуклого многогранника при отображении $\mathcal{F}_{\mathcal{P}}$.

\section{Приложение А. Полиномиальность колец многогранников}

В этом разделе равенство $F=G$ граней $F \subset P$ и $G \subset Q$ означает, что эти грани соответствуют друг другу при заданной комбинаторной эквивалентности многогранников $P$ и $Q$.

УТВЕРЖДЕНИЕ А.1. Х-колъцо многогранников $\mathcal{P}$ является колъцом полиномов от неразложимых относительно прямого произведения многогранников. 
ДокАЗАтельство. Докажем, что любой комбинаторный многогранник $P$ положительной размерности может быть единственным, с точностью до перестановки сомножителей, образом представлен в виде произведения неразложимых многогранников положительных размерностей.

Ясно, что такое разложение существует. Пусть $P=P_{1} \times \cdots \times P_{k} \simeq Q_{1} \times$ $\cdots \times Q_{s}$. Фиксируем вершину $\boldsymbol{v}=\boldsymbol{v}_{1} \times \cdots \times \boldsymbol{v}_{k}=\boldsymbol{w}_{1} \times \cdots \times \boldsymbol{w}_{s}$, где $\boldsymbol{v}_{i} \in P_{i}$ и $\boldsymbol{w}_{j} \in Q_{j}$. Грани вида $\boldsymbol{v}_{1} \times \cdots \times P_{i} \times \cdots \times \boldsymbol{v}_{k}$ и $\boldsymbol{w}_{1} \times \cdots \times Q_{j} \times \cdots \times \boldsymbol{w}_{s}$ являются максимальными $\times$-неразложимыми гранями многогранника $P$, содержащими вершину $\boldsymbol{v}$, поэтому они взаимно однозначно соответствуют друг другу при комбинаторной эквивалентности. Следовательно, $k=s$ и $P_{i} \simeq Q_{i}, i=1, \ldots, k$ с точностью до порядка множителей.

Так как $\mathcal{P}$ - свободная абелева группа, порожденная комбинаторными многогранниками, и каждый многогранник можно представить единственным образом в виде монома от неразложимых многогранников, то $\mathcal{P}$ - кольцо полиномов от неразложимых многогранников. Утверждение А.1 доказано.

Подобным образом доказывается теорема о структуре кольца $\mathcal{R} \mathcal{P}$.

УТВЕРЖДЕНИЕ А.2. *-колъцо многогранников $\mathcal{R} \mathcal{P}$ является колъцом полиномов от неразложимых относительно джойна многогранников.

ДокАЗАТЕЛЬСтво. Докажем, что любой непустой комбинаторный многогранник $P$ может быть единственным, с точностью до перестановки сомножителей, образом представлен в виде произведения непустых неразложимых многогранников.

Ясно, что такое разложение существует. Пусть $P_{1} * \cdots * P_{k} \simeq Q_{1} * \cdots * Q_{s}$. Грани $\varnothing * \cdots * P_{i} * \cdots * \varnothing$ и $\varnothing * \cdots * Q_{j} * \cdots * \varnothing$ являются максимальными *-неразложимыми гранями многогранника $P$, поэтому они взаимно однозначно соответствуют друг другу при комбинаторной эквивалентности. Следовательно, $k=s$ и $P_{i} \simeq Q_{i}, i=1, \ldots, k$, с точностью до порядка множителей.

Окончание доказательства такое же, как в утверждении А.1.

\section{Приложение В. Кольцо флаговых чисел}

Рассмотрим алгебру Хопфа $\mathcal{R}^{*}$, градуированно двойственную алгебре РотаХопфа $\mathcal{R}$. Умножение в ней задается сверткой:

$$
\varphi_{1} \star \varphi_{2}(T)=\sum_{x \in T} \varphi_{1}([\hat{0}, x]) \varphi_{2}([x, \hat{1}]), \quad \text { где } \varphi_{1}, \varphi_{2} \in \mathcal{R}^{*} \text { и } T \in \mathcal{R}
$$

В $\mathcal{R}^{*}$ содержится абелева подгруппа $\mathbf{F}=\sum \mathbf{F}^{2 n}, n \geqslant 0$, где группа $\mathbf{F}^{2 n}$ состоит из линейных функций вида $\sum \alpha_{S} f_{S}^{n}, S \subseteq[0, n-2]$, ставящих в соответствие частично упорядоченному множеству $T$ сумму $\sum \alpha_{S} f_{S}(T), S \subseteq[0, n-2]$, если $\rho(T)=n$, и 0 в противном случае (см. [40], [31]).

УТВЕРЖДЕНИЕ В.1. Имеем: $f_{S_{1}}^{n_{1}} \star f_{S_{2}}^{n_{2}}=f_{S_{1} \cup\left\{n_{1}\right\} \cup S_{2}+n_{1}}^{n_{1}+n_{2}}$, поэтому группа $\mathbf{F}$ является подкольиом в $\mathcal{R}^{*}$. 
ДокАЗАТЕЛЬСтво. Действительно,

$$
f_{S_{1}}^{n_{1}} \star f_{S_{2}}^{n_{2}}(T)=\sum_{x \in T: \rho(x)=n_{1}} f_{S_{1}}^{n_{1}}([\hat{0}, x]) f_{S_{2}}^{n_{2}}([x, \hat{1}])=f_{S_{1} \cup\left\{n_{1}\right\} \cup S_{2}}^{n_{1}+n_{2}}(T) .
$$

ОПРЕДЕЛЕНИЕ В.2. Кольцо $\mathbf{F}$ называется кольцом флаговых чисел.

Кольцо F, по существу, было введено в работе [40]. Оно подробно исследовалось в работе [31]. См. также [54].

В [31] было доказано (см. следствие 2.16 настоящего обзора), что аддитивно $\mathbf{F}$ является свободной абелевой группой с базисом $\left\{f_{S}^{n}, n \geqslant 0, S \subseteq[0, n-2]\right\}$. Легко видеть, что кольцо $\mathbf{F}$ порождается функциями $f_{\varnothing}^{n}$.

УтВЕРЖДЕНИЕ В.3. Отображение $\vartheta: \mathcal{D}(\mathcal{R}) \rightarrow \mathbf{F}$, задаваемое формулой $\langle\vartheta(D), T\rangle=\varepsilon(D T)$, является изоморфизмом колеи, при котором оператор $d_{k}$ переходит в функиию $f_{\varnothing}^{k}$.

ДокАЗАТЕЛЬСтво. Из утверждения В.1 и линейной независимости над $\mathbb{Q}$ функций $\left\{f_{S}^{n}, n \geqslant 0, S \subseteq[0, n-2]\right\}$ вытекает, что функции $\left\{f_{\varnothing}^{n}, n \geqslant 0\right\}$ алгебраически независимы. Поэтому в силу утверждения 2.24 достаточно показать, что отображение $\vartheta$ является гомоморфизмом колец. Действительно, для композиции $\omega=\left(j_{1}, \ldots, j_{k}\right),|\omega|=n$, оператора $D_{\omega}=d_{j_{1}} \cdots d_{j_{k}}$ и частично упорядоченного множества $T \in \mathcal{R}^{2 n}$ имеем:

$$
\left\langle\vartheta\left(D_{\omega}\right), T\right\rangle=\sum_{\hat{0}=x_{0}<x_{1}<\cdots<x_{k}=\hat{1}, \rho\left(x_{i}\right)-\rho\left(x_{i-1}\right)=j_{i}} 1=f_{\varnothing}^{j_{1}} \star \cdots \star f_{\varnothing}^{j_{k}}(T),
$$

откуда легко следует нужный результат.

ОПРЕДЕлЕниЕ В.4. Кольцо $(\mathcal{R} / \mathrm{Ker} \mathrm{F}) \simeq \mathrm{F}(\mathcal{R})=\mathcal{Q}$ sym назовем колъцом флаговых векторов частично упорядоченных множеств.

СлеДСтвиЕ В.5. Имеем $\mathbf{F} \simeq \mathcal{D}(\mathcal{R})$ u $\mathrm{F}(\mathcal{R}) \simeq \mathcal{D}^{*}(\mathcal{R})$. Таким образом, кольиа флаговых чисел и флаговых векторов частично упорядоченных множеств изоморфны алгебрам Хопфа, двойственным друг к другу.

Теперь рассмотрим случай многогранников.

ОПРЕДЕЛЕНИЕ В.6. Кольцо $\mathbf{F}(\mathcal{R P})=\left.\mathbf{F}\right|_{L(\mathcal{R P})}$ назовем кольщом флаговых чисел многогранников.

Имеем $\left.\mathcal{D}(\mathcal{R})\right|_{L(\mathcal{R P})}=\mathcal{D}(\mathcal{R P})$.

УТВЕРЖДЕНИЕ В.7. Отображение $\vartheta$ индуцирует канонический кольщевой изоморфизм $\mathcal{D}=\mathcal{D}(\mathcal{R} \mathcal{P}) \simeq \mathbf{F}(\mathcal{R P})$.

СледСТВиЕ В.8. Имеем $\mathbf{F}(\mathcal{R P}) \simeq \mathcal{D} u \mathcal{F}_{\varepsilon}(\mathcal{R P}) \simeq \mathcal{D}^{*}$. Таким образом, кольиа флаговых чисел и флаговых векторов многогранников изоморфны алгебрам Хопфа, двойственным друг $к$ другу.

Имеет место коммутативная диаграмма

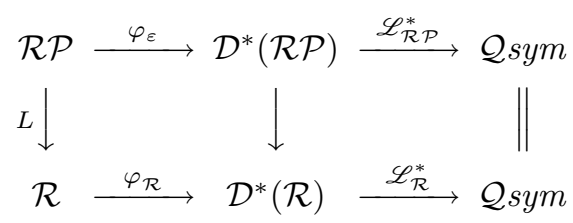


где $\left\langle\varphi_{\mathcal{R}}(T), D\right\rangle=\varepsilon(D T)$, отображение $\mathscr{L}_{\mathcal{R}}^{*}$ является изоморфизмом, $\mathscr{L}_{\mathcal{R}}^{*} \varphi_{\varepsilon}=$ $* \mathcal{F}_{\varepsilon}$ и $\mathscr{L}_{\mathcal{R}}^{*} \varphi_{\mathcal{R}}=\mathrm{F}$.

Структурная теорема 5.1 настоящего обзора в случае алгебры $\mathbf{F} \otimes \mathbb{Q} \simeq \mathcal{D} \otimes \mathbb{Q}$ была доказана в [31].

\section{Приложение С. Алгебра Стембриджа}

Для каждого множества $S \subset[0, n-1]$, не содержащего двух подряд идущих чисел и нуля, определим квазисимметрическую функцию

$$
\theta_{S}=\sum_{S^{\prime}: S \subseteq S^{\prime} \cup S^{\prime}+1} 2^{\left|S^{\prime}\right|+1} M_{S^{\prime}}, \quad S^{\prime} \subseteq[0, n-1],
$$

где для множества $S^{\prime}=\left\{a_{1}, \ldots, a_{k}\right\}$ имеем: $M_{S^{\prime}}=M_{\left(a_{1}+1, \ldots, a_{k}-a_{k-1}, n-a_{k}\right)}$. Дж. Стембридж в [28] показал, что абелева группа $\Pi \subset \mathcal{Q} s y m$, порожденная функциями $\theta_{S}$, является подкольцом, причем $\mathrm{rk}^{2 n}=c_{n-1}$. Согласно [55] кольцо П является подалгеброй Хопфа.

ОПредЕЛЕНиЕ С.1. Будем называть алгебру Хопфа П алгеброй Cmембриджа.

Алгебра П была введена в [28] для исследования так называемых обогащеннъх Р-разбиений. Можно показать (см. [56], см. также приложение Е), что алгебра $\mathscr{L}^{*}\left(\mathcal{D}^{*}\right) \otimes \mathbb{Q}=\mathscr{R}^{*}\left(\mathcal{D}^{*}\right) \otimes \mathbb{Q} \subset \mathcal{Q}$ sym $\otimes \mathbb{Q}$, задаваемая условиями следствия 7.7, совпадает с алгеброй Стембриджа $\Pi \otimes \mathbb{Q}$. В [14] рассматриваются алгебры Хопфа над полем $\mathbb{k}$ с фиксированным кольцевым гомоморфизмом в $\mathbb{k}$, вводится понятие четной и нечетной подалгебры и показывается, что $\Pi \otimes \mathbb{Q}$ является нечетной подалгеброй алгебры Хопфа $\mathcal{Q} s y m \otimes \mathbb{Q}$ с отображением $\zeta_{\mathcal{Q} s y m}$, задаваемым рядом $\sum Z_{i}=\Phi(1)$. Впервые двойственность между алгеброй Стембриджа $\Pi \otimes \mathbb{Q}$ и алгеброй Хопфа флаговых чисел $\mathbf{F} \otimes \mathbb{Q}$ была доказана в работе [56].

\section{Приложение D. Операторы Пьери}

Операторы Пьери были введены в [56]. Здесь мы опишем их связь с операторами $d_{k}$ (см. п. 1.3 и определение 1.48 ).

ОПРЕДЕЛЕНИЕ D.1. Для частично упорядоченного множества $T \in \mathcal{R}$ рассмотрим свободную абелеву группу $\mathbb{Z} T$, порожденную элементами $\{t \in T\}$. Для данного $k>0$ (правым) оператором Пьери называется любое линейное отображение $\bar{z}_{k}: \mathbb{Z} T \rightarrow \mathbb{Z T}$, согласованное со структурой порядка на $T$, т. е. для $x \in T$ элемент $x \bar{z}_{k}$ является линейной комбинацией элементов $y \in T$ таких, что $x<y$ и $\rho(y)-\rho(x)=k$. Имеем $\operatorname{deg} \bar{z}_{k}=2 k$.

Для частично упорядоченного множества $T$ семейства операторов Пьери $\left\{\bar{z}_{k}, k>0\right\}$ взаимно однозначно соответствуют структурам градуированного правого $\mathcal{Z}$-модуля на группе $\mathbb{Z} T$, согласованным со структурой порядка на $T$. 
Для любой такой структуры коэффициент при $y$ в выражении $x Z$, где $Z \in \mathcal{Z}$, является линейной функцией на $\mathcal{Z}$, принадлежащей градуированно двойственной алгебре Хопфа $\mathcal{Z}^{*}=\mathcal{Q} s y m$. Эта функция равна нулю, если не выполнено условие $x \leqslant y$. Таким образом, мы получаем набор квазисимметрических функций $K_{[x, y]}$, взаимно однозначно соответствующих интервалам $[x, y]$ в $T$.

ОПРедЕЛЕНИЕ D.2. Для частично упорядоченного множества $T \in \mathcal{R}$ рассмотрим свободную градуированную абелеву группу $\mathcal{H} T$, порожденную прямыми произведениями непустых интервалов $[x, y]$ множества $T$ при отождествлении $[x, x]=1$ для всех $x \in T$.

УТВЕРЖДЕНИЕ D.3 [56]. Для любого частично упорядоченного множества $T \in \mathcal{R}$ группа $\mathcal{H} T$ является градуированной алгеброй Хопфа.

Для любого семейства операторов Пьери на множестве $T \in \mathcal{R}$ коумножение на $\mathcal{Z}$ и каноническое действие алгебры $\mathcal{Z} \otimes \mathcal{Z}$ на $\mathbb{Z} T \otimes \mathbb{Z} T=\mathbb{Z}(T \times T)$ индуцируют каноническое действие алгебры $\mathcal{Z}$ на $\mathbb{Z}(T \times T)$. Итерируя эту конструкцию, получаем действие алгебры $\mathcal{Z}$ на $\mathbb{Z} T^{k}$. Так как любое произведение интервалов множества $T$ является интервалом в некотором множестве $T^{k}$, то мы можем распространить определение функции $K$ на образующие группы $\mathcal{H} T$, а затем по линейности на всю группу $\mathcal{H} T$.

Tеорема D.4 [56]. Отображение $K: \mathcal{H} T \rightarrow \mathcal{Q}$ sym является гомоморфизмом алгебр Хопфа.

Рассмотрим билинейную форму $\langle\cdot, \cdot\rangle$ на $\mathcal{H} T$, индуцированную $\delta$-функцией Кронекера элементов множества $T$, и введем канонический элемент

$$
\mathscr{C}=\sum_{\lambda} Z_{\lambda} \otimes Z_{\lambda}^{*} \in \sum_{n \geqslant 0} \mathcal{Z}^{2 n} \otimes \mathcal{Q} \operatorname{sym}^{2 n},
$$

где $\left\{Z_{\lambda}\right\}-$ произвольный градуированный базис абелевой группы $\mathcal{Z}$ и $\left\{Z_{\lambda}^{*}\right\}-$ двойственный базис абелевой группы $\mathcal{Q} s y m$. Этот элемент задает разложение тождественного оператора $\mathcal{Z} \rightarrow \mathcal{Z}$ и, очевидно, не зависит от выбора базиса в $\mathcal{Z}$. Такие элементы играют важную роль в общей теории алгебр Хопфа. В данном конкретном случае алгебры $\mathcal{Z}$ в [25] он назван элементом Коши.

Tеорема D.5 [56]. Для любого семейства операторов Пъери на множестве $T \in \mathcal{R}$ и элементов $x, y \in T$ имеет место формула: $K_{[x, y]}=\langle x \mathscr{C}, y\rangle$.

ПримеP D.6. Для любого частично упорядоченного множества $T$ рассмотрим оператор Пьери $z_{k}$, ставящий в соответствие элементу $x \in T$ сумму всех элементов $y \in T$ таких, что $y>x$ и $\rho(y)-\rho(x)=k$. В этом случае для любой композиции $\omega=\left(j_{1}, \ldots, j_{k}\right),|\omega|=\rho(y)-\rho(x)$, значение $\left\langle x Z_{\omega}, y\right\rangle$ равно числу цепочек $x=x_{0}<x_{1}<\cdots<x_{k}=y$ таких, что $\rho\left(x_{i}\right)-\rho\left(x_{i-1}\right)=j_{i}, i=1, \ldots, k$. Тогда $K_{[\hat{0}, \hat{1}]}=\mathrm{F}(T)$, где $\mathrm{F}$ - гомоморфизм Эренборга.

Операторы Пьери соответствуют представлениям алгебры $\mathcal{Z}$ на группах $\mathbb{Z} T$, $T \in \mathcal{R}$. В настоящем обзоре мы используем операторы $d_{k}$, которые соответствуют представлению алгебры Хопфа $\mathcal{Z}$ на алгебре Рота-Хопфа $\mathcal{R}$. При этом операторы $z_{k}$ отвечают операторам $\delta_{k}=* d_{k} *$ таким, что $\delta_{k}(T)=\left(d_{k} T^{*}\right)^{*}$. 


\section{Приложение Е. $c d$-индекс}

В проблеме флаговых чисел выпуклых многогранников ряд глубоких результатов получен на основе $\boldsymbol{c d}$-индекса (см. [41], [57], см. также в [31], [45], [58]-[61]), введенного Дж. Файном. Это приложение содержит результаты, связывающие $\boldsymbol{c d}$-индекс с алгебраическими конструкциями, описанными в нашем обзоре.

E.1. Определения и конструкции. Рассмотрим кольцо $\mathbb{Z}\langle\boldsymbol{a}, \boldsymbol{b}\rangle, \operatorname{deg} \boldsymbol{a}=$ $\operatorname{deg} \boldsymbol{b}=2$. Пусть $P-n$-мерный многогранник. Каждому множеству $S \subseteq$ $[0, n-1]$ сопоставим моном $\boldsymbol{u}_{S}=u_{1} \cdots u_{n}$, где $u_{i}=\boldsymbol{b}$, если $i-1 \in S$, и $u_{i}=\boldsymbol{a}$ иначе. Введем некоммутативные полиномы $\Upsilon_{P}, \Psi_{P} \in \mathbb{Z}\langle\boldsymbol{a}, \boldsymbol{b}\rangle$ по формулам

$$
\Upsilon_{P}(\boldsymbol{a}, \boldsymbol{b})=\sum_{S \subseteq[0, n-1]} f_{S} \boldsymbol{u}_{S}, \quad \Psi_{P}(\boldsymbol{a}, \boldsymbol{b})=\sum_{S \subseteq[0, n-1]} h_{S} \boldsymbol{u}_{S}=\Upsilon_{P}(\boldsymbol{a}-\boldsymbol{b}, \boldsymbol{b}),
$$

где $f_{S}$ - флаговые числа многогранника $P$ (см. п. 1.6). Отметим, что переход от полинома $\Upsilon_{P}$ к полиному $\Psi_{P}$ осуществляется по тем же формулам, что и переход от $f$ к $h$-полиному простого многогранника (см. замечание 6.12 и п. 10.3).

ОПРЕДЕЛЕНИЕ Е.1 (см. [41], [57]). Флаговым $h$-вектором многогранника $P$ назовем вектор с компонентами $h_{S}(P)=\sum_{S^{\prime} \subseteq S}(-1)^{|S|-\left|S^{\prime}\right|} f_{S^{\prime}}(P), S \subseteq[0, n-1]$.

Положим $\bar{S}=[0, n-1] \backslash S$.

УТВЕРЖДЕНИЕ Е.2. Имеем: $\Psi_{P}(\boldsymbol{a}, \boldsymbol{b})=\Psi_{P}(\boldsymbol{b}, \boldsymbol{a})$ для любого п-мерного многогранника $P$, m.e. $h_{\bar{S}}(P)=h_{S}(P)$ для любого $S \subseteq[0, n-1]$.

УтВеРЖДЕНИЕ Е.3 (Дж. Файн). Некоммутативный полином $Q(\boldsymbol{a}, \boldsymbol{b})=$ $\sum q_{S} \boldsymbol{u}_{S}, S \subseteq[0, n-1]$, может быть записан в виде некоммутативного полинома $L(\boldsymbol{c}, \boldsymbol{d})$, где $\boldsymbol{c}=\boldsymbol{a}+\boldsymbol{b} u \boldsymbol{d}=\boldsymbol{a} \boldsymbol{b}+\boldsymbol{b} \boldsymbol{a}$, тогда и только тогда, когда коэфбициенты полинома $Q(\boldsymbol{a}+\boldsymbol{b}, \boldsymbol{b})$ удовлетворяют соотношениям БайерБиллера (26).

Ниже мы получим доказательство этого факта в терминах $\times$-кольца выпуклых многогранников (см. следствие Е.12).

ЗАмечАниЕ Е.4. В утверждении Е.3 говорится об универсальном свойстве соотношений Байер-Биллера, которое дает нетривиальное обобщение на некоммутативный случай классического результата коммутативной алгебры о том, что любой симметрический полином может быть записан как полином от элементарных симметрических функций.

СЛЕДСТВИЕ Е.5. Для любого многогранника $P$ определен некоммутативньй полином $\Xi(\boldsymbol{c}, \boldsymbol{d})(P) \in \mathbb{Z}\langle\boldsymbol{c}, \boldsymbol{d}\rangle, \operatorname{deg} \boldsymbol{c}=2, \operatorname{deg} \boldsymbol{d}=4$, mакой, что $\Psi_{P}(\boldsymbol{a}, \boldsymbol{b})=$ $\Xi(\boldsymbol{c}, \boldsymbol{d})(P)$.

Полином $\Xi(\boldsymbol{c}, \boldsymbol{d})(P)$ называется $\boldsymbol{c d}$-индексом многогранника $P$.

ЗАмЕчАниЕ Е.6. Все определения годятся для любого частично упорядоченного множества $T \in \mathcal{R}$. В этом случае $\boldsymbol{c d}$-индекс множества $T$ существует тогда и только тогда, когда флаговые числа этого множества удовлетворяют соотношениям Байер-Биллера. В частности, $\boldsymbol{c d}$-индекс определен, если частично упорядоченное множество является эйлеровым. 
Обозначим через $[\boldsymbol{w}]_{P}$ коэффициент при слове $\boldsymbol{w}=\boldsymbol{c}^{l_{1}} \boldsymbol{d} \boldsymbol{c}^{l_{2}} \boldsymbol{d} \cdots \boldsymbol{c}^{l_{k}} \boldsymbol{d} \boldsymbol{c}^{r}$ в $\boldsymbol{c} \boldsymbol{d}$ индексе $\Xi(P)$. Тогда $\Xi(\boldsymbol{c}, \boldsymbol{d})(P)=\sum[\boldsymbol{w}]_{P} \boldsymbol{w}, \operatorname{deg} \boldsymbol{w}=2 \operatorname{dim} P$.

Приведем один из наиболее общих результатов о линейных неравенствах в проблеме флаговых векторов.

Теорема Е.7 [57]. Пусть $P^{n}$ - выпуклый многогранник. Тогда для всех слов $\boldsymbol{w}$ имеем $[\boldsymbol{w}]_{P} \geqslant 0$.

Доказательство этого факта впервые было получено при помощи индукции в [57] на основе понятия $S$-шеллинга. Геометрическое доказательство этого факта (см. [45]) использует геометрическую интерпретацию $\boldsymbol{c d}$-индекса, аналогичную описанию (теорема А.Г. Хованского, см. [9]) коэффициента $h_{i}(P)$ простого многогранника $P \subset \mathbb{R}^{n}$ как числа вершин индекса $i$ для линейного функционала общего положения на пространстве $\mathbb{R}^{n}$.

Для слова $\boldsymbol{w}=\boldsymbol{c}^{l_{1}} \boldsymbol{d} \boldsymbol{c}^{l_{2}} \boldsymbol{d} \cdots \boldsymbol{c}^{l_{k}} \boldsymbol{d} \boldsymbol{c}^{r}, \operatorname{deg} \boldsymbol{w}=2 n$, введем множество $S_{\boldsymbol{w}}=$ $\left\{l_{1}+1, l_{1}+l_{2}+3, \ldots, l_{1}+\cdots+l_{k}+2 k-1\right\}$ и положим

$$
\theta_{\boldsymbol{w}}=\sum_{S^{\prime}: S_{\omega} \in S^{\prime} \cup\left(S^{\prime}+1\right)} 2^{\left|S^{\prime}\right|+1} M_{S^{\prime}}, \quad S^{\prime} \subseteq[0, n-1] .
$$

Положим $|\boldsymbol{w}|_{\boldsymbol{d}}=k$.

УТВЕРЖДЕНИЕ Е.8 (см. [38]). Пусть $P-n$-мерный выпуклый многогранник. Для гомоморфизма Эренборга имеет место формула

$$
\mathrm{F}(L(P))=\sum_{S \subseteq[0, n-1]} f_{S} M_{S}=\sum_{\boldsymbol{w}: \operatorname{deg} \boldsymbol{w}=2 n} \frac{1}{2^{|\boldsymbol{w}|_{d}+1}}[\boldsymbol{w}]_{P} \theta_{\boldsymbol{w}} .
$$

ДокаЗАТЕЛЬСтво. Имеем:

$$
\begin{aligned}
\sum f_{S} \boldsymbol{u}_{S}= & \Psi_{P}(\boldsymbol{a}+\boldsymbol{b}, \boldsymbol{b})=\Xi\left(\boldsymbol{a}+2 \boldsymbol{b}, \boldsymbol{a} \boldsymbol{b}+\boldsymbol{b} \boldsymbol{a}+2 \boldsymbol{b}^{2}\right)(P) \\
= & \sum_{\operatorname{deg} \boldsymbol{w}=2 n}[\boldsymbol{w}]_{P}(\boldsymbol{a}+2 \boldsymbol{b})^{l_{1}}\left(\boldsymbol{a} \boldsymbol{b}+\boldsymbol{b} \boldsymbol{a}+2 \boldsymbol{b}^{2}\right)(\boldsymbol{a}+2 \boldsymbol{b})^{l_{2}} \cdots(\boldsymbol{a}+2 \boldsymbol{b})^{l_{k}} \\
& \times\left(\boldsymbol{a} \boldsymbol{b}+\boldsymbol{b} \boldsymbol{a}+2 \boldsymbol{b}^{2}\right)(\boldsymbol{a}+2 \boldsymbol{b})^{r} .
\end{aligned}
$$

Для множества $S^{\prime} \subseteq[0, n-1]$ моном $\boldsymbol{u}_{S^{\prime}}$ встречается в разложении монома $\boldsymbol{w}$ по $\boldsymbol{a}, \boldsymbol{b}$ тогда и только тогда, когда $S_{\boldsymbol{w}} \subseteq S^{\prime} \cup\left(S^{\prime}+1\right)$. В этом случае коэффициент при $\boldsymbol{u}_{S^{\prime}}$ равен $2^{\left|S^{\prime}\right|-\left|S_{\boldsymbol{w}}\right|}=\frac{2^{\left|S^{\prime}\right|+1}}{2^{|\boldsymbol{w}|_{d}+1}}$. Тогда

$$
f_{S^{\prime}}=\sum_{\boldsymbol{w}: S_{\boldsymbol{w}} \subseteq S^{\prime} \cup\left(S^{\prime}+1\right)} \frac{2^{\left|S^{\prime}\right|+1}}{2^{|\boldsymbol{w}|_{d}+1}}[\boldsymbol{w}]_{P}, \quad \operatorname{deg} \boldsymbol{w}=2 n,
$$

следовательно,

$$
\begin{aligned}
& \mathrm{F}(L(P))=\sum_{S^{\prime} \subseteq[0, n-1]} f_{S^{\prime}} M_{S^{\prime}}=\sum_{S^{\prime} \subseteq[0, n-1]}\left(\sum_{\substack{\boldsymbol{w}: \operatorname{deg} \boldsymbol{w}=2 n \\
S_{\boldsymbol{w}} \subseteq S^{\prime} \cup\left(S^{\prime}+1\right)}} \frac{2^{\left|S^{\prime}\right|+1}}{2^{|\boldsymbol{w}|_{d}+1}}[\boldsymbol{w}]_{P}\right) M_{S^{\prime}} \\
& =\sum_{\boldsymbol{w}: \operatorname{deg} \boldsymbol{w}=2 n} \frac{1}{2^{|\boldsymbol{w}|_{d}+1}}[\boldsymbol{w}]_{P}\left(\sum_{S^{\prime}: S_{\boldsymbol{w}} \subseteq S^{\prime} \cup\left(S^{\prime}+1\right)} 2^{\left|S^{\prime}\right|+1} M_{S^{\prime}}\right)=\sum_{\boldsymbol{w}: \operatorname{deg} \boldsymbol{w}=2 n} \frac{1}{2^{|\boldsymbol{w}|_{\boldsymbol{d}}+1}}[\boldsymbol{w}]_{P} \theta_{\boldsymbol{w}} .
\end{aligned}
$$

СлеДСтвие Е.9. Имеем: $\mathscr{R}^{*}\left(\mathcal{D}^{*}\right) \otimes \mathbb{Q}=\mathcal{F}_{\varepsilon}(\mathcal{R} \mathcal{P}) \otimes \mathbb{Q}=\Pi \otimes \mathbb{Q}$. 
E.2. $\boldsymbol{c d}$-индекс и флаговый полином. Имеет место изоморфизм абелевых групп ю: $\mathcal{Q} \operatorname{sym}[\alpha] \rightarrow \mathbb{Z}\langle\boldsymbol{a}, \boldsymbol{b}\rangle$, задаваемый соответствием

$$
\alpha^{j_{1}} M_{\left(j_{k}, \ldots, j_{2}\right)} \rightarrow \boldsymbol{a}^{j_{1}} \boldsymbol{b} \boldsymbol{a}^{j_{2}-1} \boldsymbol{b} \cdots \boldsymbol{a}^{j_{k-1}-1} \boldsymbol{b a}^{j_{k}-1} .
$$

Непосредственно из определения получаем:

УтВЕРЖДЕНИЕ Е.10. Имеем: $\wp\left[\mathcal{F}_{\mathcal{P}}(P)\right]=\Upsilon_{P}(\boldsymbol{a}, \boldsymbol{b})$.

Рассмотрим замену переменных $\boldsymbol{c}=\boldsymbol{a}+2 \boldsymbol{b}$ и $\boldsymbol{d}=\boldsymbol{a} \boldsymbol{b}+\boldsymbol{b} \boldsymbol{a}+2 \boldsymbol{b}^{2}$.

УТВЕРЖДЕНИЕ Е.11. Имеем: $\wp(g \boldsymbol{c})=\wp(g) \boldsymbol{c}, \wp(g \boldsymbol{d})=\wp(g) \boldsymbol{d}$.

ДоКАЗАТЕЛЬСТво. Используя пример 6.25, получаем:

$$
\begin{gathered}
\wp\left(\alpha^{j_{1}} \boldsymbol{c}\right)=\wp\left[\alpha^{j_{1}}\left(\alpha+2 M_{(1)}\right)\right]=\boldsymbol{a}^{j_{1}+1}+2 \boldsymbol{a}^{j_{1}} \boldsymbol{b}=\wp\left(\alpha^{j_{1}}\right) \boldsymbol{c} ; \\
\wp\left(\left[\alpha^{j_{1}} M_{\left(j_{k}, \ldots, j_{2}\right)}\right] \boldsymbol{c}\right)=\wp\left(\alpha^{j_{1}}\left(M_{\left(j_{k}+1, j_{k-1}, \ldots, j_{2}\right)}+2 M_{\left(1, j_{k}, \ldots, j_{2}\right)}\right)\right) \\
=\boldsymbol{a}^{j_{1}} \boldsymbol{b} \boldsymbol{a}^{j_{2}-1} \boldsymbol{b} \cdots \boldsymbol{b} \boldsymbol{a}^{j_{k}}+2 \boldsymbol{a}^{j_{1}} \boldsymbol{b} \boldsymbol{a}^{j_{2}-1} \boldsymbol{b} \cdots \boldsymbol{b} \boldsymbol{a}^{j_{k}-1} \boldsymbol{b}=\wp\left(\alpha^{j_{1}} M_{\left(j_{k}, \ldots, j_{2}\right)}\right) \boldsymbol{c} ; \\
\wp\left(\alpha^{j_{1}} \boldsymbol{d}\right)=\wp\left[\alpha^{j_{1}}\left(\alpha M_{(1)}+M_{(2)}+2 M_{(1,1)}\right)\right]=\boldsymbol{a}^{j_{1}+1} \boldsymbol{b}+\boldsymbol{a}^{j_{1}} \boldsymbol{b} \boldsymbol{a}+2 \boldsymbol{a}^{j_{1}} \boldsymbol{b}^{2}=\wp\left(\alpha^{j_{1}}\right) \boldsymbol{d} ; \\
\wp\left(\left[\alpha^{j_{1}} M_{\left(j_{k}, \ldots, j_{2}\right)}\right] \boldsymbol{d}\right)=\wp\left(\alpha^{j_{1}}\left(M_{\left(1, j_{k}+1, j_{k-1}, \ldots, j_{2}\right)}+M_{\left(2, j_{k}, \ldots, j_{2}\right)}+2 M_{\left(1,1, j_{k}, \ldots, j_{2}\right)}\right)\right) \\
=\boldsymbol{a}^{j_{1}} \boldsymbol{b} \boldsymbol{a}^{j_{2}-1} \boldsymbol{b} \cdots \boldsymbol{b} \boldsymbol{a}^{j_{k}} \boldsymbol{b}+\boldsymbol{a}^{j_{1}} \boldsymbol{b} \boldsymbol{a}^{j_{2}-1} \boldsymbol{b} \cdots \boldsymbol{b} \boldsymbol{a}^{j_{k}-1} \boldsymbol{b a}+2 \boldsymbol{a}^{j_{1}} \boldsymbol{b} \boldsymbol{a}^{j_{2}-1} \boldsymbol{b} \cdots \boldsymbol{b} \boldsymbol{a}^{j_{k}-1} \boldsymbol{b}^{2} \\
=\wp\left(\alpha^{j_{1}} M_{\left(j_{k}, \ldots, j_{2}\right)}\right) \boldsymbol{d} .
\end{gathered}
$$

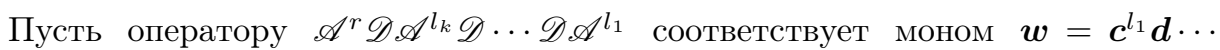
$\boldsymbol{d}^{l_{k}} \boldsymbol{d} \boldsymbol{c}^{r}$ при антиизоморфизме $\mathbb{Z}\langle\mathscr{A}, \mathscr{D}\rangle \rightarrow \mathbb{Z}\langle\boldsymbol{c}, \boldsymbol{d}\rangle$. Используя разложение

$$
\pi_{\mathscr{A} \mathscr{D}}(P)=\sum[W]_{P}(W \mathrm{pt})
$$

(см. определение 1.91), мы получим:

$$
\begin{aligned}
\mathcal{F}_{\mathcal{P}}(P) & =\mathcal{F}_{\mathcal{P}}\left(\pi_{\mathscr{A} \mathscr{D}}(P)\right)=\mathcal{F}_{\mathcal{P}}\left(\sum[W]_{P}(W \mathrm{pt})\right)=\sum[W]_{P} \mathcal{F}_{\mathcal{P}}(W \mathrm{pt}) \\
& =\sum[W]_{P}\left(W_{\mathcal{P}} 1\right)=\sum[W]_{P}(1 \boldsymbol{w}), \quad \text { где } W_{\mathcal{P}} 1=\mathcal{F}_{\mathcal{P}}(W \mathrm{pt}) .
\end{aligned}
$$

СледСтвиЕ Е.12. 1) Для любого многогранника $P$ существует $\boldsymbol{c d}$-индекс $\Xi(\boldsymbol{c}, \boldsymbol{d})(P)$.

2) $[\boldsymbol{w}]_{P}=[W]_{P}$.

3) Верно утверждение Е.3.

Доказательство. Так как $\wp(1)=1$, то для элемента $P \in \mathcal{P}^{2 n}$ получаем:

$$
\begin{aligned}
\Upsilon_{P}(\boldsymbol{a}, \boldsymbol{b}) & =\wp\left[\mathcal{F}_{\mathcal{P}}(P)\right]=\wp\left[\mathcal{F}_{\mathcal{P}}\left(\pi_{\mathscr{A} \mathscr{D}}(P)\right)\right]=\wp\left[\mathcal{F}_{\mathcal{P}}\left(\sum[W]_{P}(\operatorname{pt} \boldsymbol{w})\right)\right] \\
& =\sum[W]_{P}(1 \boldsymbol{w})=\sum[W]_{P} \boldsymbol{w}=\Xi(\boldsymbol{c}, \boldsymbol{d})(P) .
\end{aligned}
$$

Тем самым мы доказали первые два утверждения. Что касается третьего, то пусть коэффициенты полинома $Q(\boldsymbol{a}+\boldsymbol{b}, \boldsymbol{b})$ степени $2 n$ удовлетворяют соотношениям Байер-Биллера. Тогда

$$
\wp^{-1}(Q(\boldsymbol{a}+\boldsymbol{b}, \boldsymbol{b}))=\mathcal{F}_{\mathcal{P}}(P)
$$

для некоторого $P \in \mathcal{P}^{2 n}$. Следовательно, $Q(\boldsymbol{a}, \boldsymbol{b})=\Xi(\boldsymbol{c}, \boldsymbol{d})(P)$. Пусть теперь $Q(\boldsymbol{a}, \boldsymbol{b})=L(\boldsymbol{c}, \boldsymbol{d})$. В этом случае

$$
Q(\boldsymbol{a}+\boldsymbol{b}, \boldsymbol{b})=\wp[1 L(\boldsymbol{c}, \boldsymbol{d})]=\wp\left[\mathcal{F}_{\mathcal{P}}(\mathrm{pt} L(\boldsymbol{c}, \boldsymbol{d}))\right] .
$$


Следовательно, коэффициенты полинома $Q(\boldsymbol{a}+\boldsymbol{b}, \boldsymbol{b})$ удовлетворяют соотношениям Байер-Биллера.

СлеДСтвие Е.13. 1) Пустъ $P$ - n-мерный многогранник. Тогда

$$
\pi_{\mathscr{A} \mathscr{D}}(P)=\sum[W]_{P}(W \mathrm{pt}), \quad \text { əде }[W]_{P} \geqslant 0 .
$$

2) Пусть $\sum \lambda_{S} f_{S}(W \mathrm{pt}) \geqslant 0$ для всех $W, \operatorname{deg} W=2 n$. Тогда $\sum \lambda_{S} f_{S}(P) \geqslant 0$ для всех $n$-мерных многогранников $P$.

СлЕДСТвиЕ Е.14. Имеем:

$$
\mathcal{F}_{\mathcal{P}}(W \mathrm{pt})=\sum_{S: S_{\boldsymbol{w}} \subseteq S \cup(S+1)} 2^{|S|-\left|S_{\boldsymbol{w}}\right|} \alpha^{a_{1}} M_{\left(n-a_{k}, a_{k}-a_{k-1}, \ldots, a_{2}-a_{1}\right)},
$$

где $S=\left\{a_{1}, \ldots, a_{k}\right\}$, m.e. $f_{S}(W \mathrm{pt})=2^{|S|-\left|S_{\boldsymbol{w}}\right|}$, если $S_{\boldsymbol{w}} \subseteq S \cup(S+1)$, и 0 иначе.

ДокАЗАТЕЛЬСтво следует из утверждения Е.8 и следствия Е.13.

Отметим, что в разложении элемента $P=W$ pt по базису из многогранников часть коэффициентов отрицательна. Тем не менее, для любого $S$ флаговое число $f_{S}(P)$ неотрицательно.

Связь $\boldsymbol{c d}$-индекса и флагового полинома $\mathcal{F}_{\mathcal{P}}$ можно описать при помощи следующей коммутативной диаграммы:

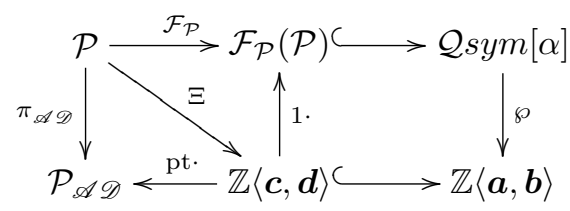

где изоморфизмы $\mathbb{Z}\langle\boldsymbol{c}, \boldsymbol{d}\rangle \rightarrow \mathcal{F}_{\mathcal{P}}(\mathcal{P})$ и $\mathbb{Z}\langle\boldsymbol{c}, \boldsymbol{d}\rangle \rightarrow \mathcal{P} \mathscr{A} \mathscr{D}$ задаются отображениями $Q(\boldsymbol{c}, \boldsymbol{d}) \rightarrow 1 Q(\boldsymbol{c}, \boldsymbol{d})$ и $Q(\boldsymbol{c}, \boldsymbol{d}) \rightarrow \operatorname{pt} Q(\boldsymbol{c}, \boldsymbol{d})$ и все коэффициенты как у $\Xi(P)$, так и у $\pi_{\mathscr{A} \mathscr{D}}(P)$ неотрицательны для любого многогранника.

E.3. Геометрический смысл $\boldsymbol{c d}$-индекса. Следующая конструкция $\boldsymbol{c d}$ индекса дана в [45].

Конструкция Е.15. Для выпуклого многогранника $P^{n} \subset \mathbb{R}^{n}$ введем многогранник усечения $T(P)$ следующим образом: сначала срежем вершины многогранника $P$, параллельно перенося опорные гиперплоскости к вершинам на глубину $\varepsilon$, и пометим каждую из получившихся гиперграней числом 0. Затем срежем первоначальные ребра многогранника $P$, параллельно перенося опорные гиперплоскости к ребрам на глубину $\varepsilon^{2}$, и пометим каждую из получившихся гиперграней числом 1. Повторяя эту процедуру, в конце концов мы срежем каждую из первоначальный гиперграней многогранника $P$, параллельно перенося гиперплоскости, содержащие гиперграни, на глубину $\varepsilon^{n}$, и пометим каждую из получившихся гиперграней числом $n-1$. Получившийся многогранник называется многогранником усечения и обозначается $T(P)$.

Многогранник $T(P)$ является простым, причем его грани взаимно однозначно соответствуют цепочкам граней многогранника $P$. Для грани $G \subseteq T(P)$ положим $\sigma(G)=S$, где $S$ - множество меток гиперграней, содержащих грань $G$. 
Выберем вектор $\boldsymbol{p} \in\left(\mathbb{R}^{n}\right)^{*}$ такой, что функция $\langle\boldsymbol{p}, \boldsymbol{x}\rangle$ принимает различные значения на всех вершинах, возникающих на всех шагах срезки многогранника $P$. Следующие рассуждения верны для всех достаточно малых $\varepsilon$.

Для грани $G \subseteq T(P), \operatorname{dim} G>0$, положим $j=\min \{i: i \notin \sigma(G)\}$ и пусть $w$ - вершина с наибольшим значением функции $\langle\boldsymbol{p}, \boldsymbol{x}\rangle$. Определим верхнюю грань многогранника $G$ как его единственную гипергрань $\tau(G)$, содержащую вершину $w$, для которой $\sigma(\tau(G))=\sigma(G) \cup\{j\}$. Аналогично, пусть $w^{\prime}$ - вершина с наименьшим значением функции $\langle\boldsymbol{p}, \boldsymbol{x}\rangle$. Определим нижнюю грань многогранника $G$ как его единственную гипергрань $\beta(G)$, содержащую вершину $w^{\prime}$, для которой $\sigma(\beta(G))=\sigma(G) \cup\{j\}$.

Для вершины $v \in P$ обозначим через $Q_{v}$ гипергрань, возникшую при ее срезании, и через $T\left(Q_{v}\right)$ многогранник усечения, индуцированный вложением $Q_{v} \subset T(P)$. Пусть $H_{v}=\left\{\boldsymbol{x} \in \mathbb{R}^{n}:\langle\boldsymbol{p}, \boldsymbol{x}\rangle=q_{v}\right\}$ - гиперплоскость, проходящая через вершину $v$ и $H_{v}^{+}$- открытое полупространство $\left.\left\{\boldsymbol{x} \in \mathbb{R}^{n}:\langle\boldsymbol{p}, \boldsymbol{x}\rangle\right\rangle q_{v}\right\}$, а $H_{v}^{-}$- открытое полупространство $\left\{\boldsymbol{x} \in \mathbb{R}^{n}:\langle\boldsymbol{p}, \boldsymbol{x}\rangle\left\langle q_{v}\right\}\right.$. Назовем грани многогранника $T\left(Q_{v}\right)$ высокими, средними и низкими в зависимости от того, лежат ли они в полупространстве $H_{v}^{+}$, пересекают гиперплоскость $H_{v}$ или лежат в полупространстве $H_{v}^{-}$. Пусть $R_{v}$ - многогранник $Q_{v} \cap H_{v}$. Если $Q_{v} \cap H_{v} \neq \varnothing$, то $R_{v}$ имеет размерность $n-2$. (Он будет пустым только в случае минимальной и максимальной вершины.) Пусть $T\left(R_{v}\right)$ - многогранник усечения, индуцированный усечением многогранника $P$, т. е. $T\left(R_{v}\right)=T\left(Q_{v}\right) \cap H_{v}$. Тогда грани многогранника $T\left(R_{v}\right)$ являются в точности пересечениями гиперплоскости $H_{v}$ со средними гранями многогранника $T\left(Q_{v}\right)$. Отметим, что $0 \in \sigma(G)$ для грани $G \subseteq T(P)$ тогда и только тогда, когда $G$ принадлежит некоторому многограннику $T\left(Q_{v}\right)$.

ЛЕмма Е.16. Для каждой грани $G \subseteq T(P)$ такой, что $0 \notin \sigma(G)$, верхняя грань $\tau(G)$ является низкой гранъю некоторого многогранника $T\left(Q_{v}\right)$, а нижняя грань $\beta(G)$ является высокой гранъю некоторого (другого) многогранника $T\left(Q_{v^{\prime}}\right)$. Более того, каждая высокая (низкая) грань любого многогранника $T\left(Q_{v}\right)$ является нижней (верхней) гранвю ровно для одной грани $G$ многогранника $T(P), 0 \notin \sigma(G)$.

Далее разбиением многогранника мы будем называть разбиение множества его граней на непересекающиеся подмножества, называемые блоками. Пусть дан многогранник $P$ и соответствующий ему многогранник усечения $T(P)$. Разбиение многогранника $T(P)$ на блоки мы будем называть разбиением K. Ли, если выполняются следующие условия:

P1. Каждой вершине $v$ многогранника $P$ соответствует (быть может, пустой) набор $\mathcal{B}_{v}(P)$ блоков разбиения.

Р2. Если $n>0$, то каждая грань $G$ такая, что $0 \notin \sigma(G)$, находится в том же блоке, что и ее верхняя грань $\tau(G)$.

Р3. Пусть $n>0$ и $H$ - гиперплоскость вида $\langle\boldsymbol{p}, \boldsymbol{x}\rangle=\lambda$, не пересекающая никакую грань $T\left(Q_{v}\right)$. Тогда для любой вершины $v$ многогранника $P$, лежащей в $H^{+}$, все грани в блоках из набора $\mathcal{B}_{v}(P)$ тоже лежат в $H^{+}$.

Следующая рекуррентная конструкция разбиения многогранника $T(P)$ была предложена в работе [45]. 
Конструкция Е.17. Шаг 0. Если $\operatorname{dim} P=0$, то многогранник $T(P)$ является единственной вершиной $v$ и $\mathcal{B}_{v}(P)$ состоит из единственного блока $\{v\}$. Пусть теперь $\operatorname{dim} P>0$.

Шаг 1. Для каждой грани $G \subseteq T(P)$ такой, что $0 \notin \sigma(G)$, образуем "предблок" $\{G, \tau(G), \beta(G)\}$, состоящий из самой грани $G$ и ее верхней и нижней граней. На этом шаге, согласно лемме Е.16, все грани многогранника $T(P)$, за исключением средних граней всевозможных многогранников $T\left(Q_{v}\right)$, отнесены к некоторым предблокам.

Шаг 2. Для каждой вершины $v$ и средней грани $G \subseteq T\left(Q_{v}\right)$ поместим $G$ в предблок, содержащий верхнюю грань $\tau(G)$, которая будет высокой гранью многогранника $T\left(Q_{v}\right)$. На этом шаге все грани многогранника $T(G)$ отнесены к предблокам, которые, в свою очередь, взаимно однозначно соответствуют высоким граням. При этом средние грани лежат в разных предблоках.

Шаг 3. Для вершины $v \in P$ рассмотрим рекуррентно определенное разбиение $\mathcal{B}\left(R_{v}\right)$ многогранника $T\left(R_{v}\right)$ (оно будет пустым, если многогранник $R_{v}$ пуст). Пусть $B$ - некоторый блок этого разбиения. Каждая грань в блоке $B$ соответствует некоторой средней грани многогранника $T\left(Q_{v}\right)$. Объединим предблоки, содержащие эти средние грани, в блок $B^{\prime}$ и поместим $B^{\prime}$ в $\mathcal{B}_{v}(P)$.

Шаг 4. Для вершины $v \in P$ рассмотрим рекуррентно определенное разбиение $\mathcal{B}\left(Q_{v}\right)$ многогранника $T\left(Q_{v}\right)$. Для каждой вершины $w$ многогранника $Q_{v}$, лежащей в полупространстве $H_{v}^{+}$, рассмотрим набор блоков $\mathcal{B}_{w}\left(Q_{v}\right)$ разбиения $\mathcal{B}\left(Q_{v}\right)$, ассоциированных с вершиной $w$. Пусть $B$ - блок из $\mathcal{B}_{w}\left(Q_{v}\right)$ (если такой есть). По свойству Р3 грани из блока $B$ являются некоторыми высокими гранями многогранника $T\left(Q_{v}\right)$. Объединим предблоки, содержащие эти высокие грани, в блок $B^{\prime}$ и поместим $B^{\prime}$ в $\mathcal{B}_{v}(P)$. После того как эта процедура будет проделана для каждой вершины $v$ многогранника $P$, все предблоки окажутся объединенными в блоки и $\mathcal{B}(P)=\bigcup_{v} \mathcal{B}_{v}(P)$.

Из конструкции легко вывести следующий результат.

Теорема Е.18. Конструкция Е.17 дает разбиение К. Ли многогранника $T(P)$.

Опишем рекуррентный метод вычисления $\boldsymbol{c d}$-индекса при помощи параллельного движения гиперплоскости на основе конструкции Е.17. При этом получится разложение $\Xi(P)=\sum \Xi_{v}(P), v \in \operatorname{vert}(P)$, где слагаемое $\Xi_{v}(P)$ определяется блоком $B_{v}(P)$. Этот метод является двойственным к методу вычисления полинома $\Xi(P)$, предложенному Р. Стенли в [57].

Tеорема Е.19. Пусть $P$ - выпуклый $n$-мернъй многогранник. Тогда:

1) если $n=0$, то $P$ имеет одну вершину $v$ и $\Xi_{v}(P)=\Xi(P)=1$;

2) если $n>0$, mo

$$
\Xi_{v}(P)=\boldsymbol{d} \Xi\left(R_{v}\right)+\sum_{w \in \operatorname{vert}\left(Q_{v}\right) \cap H_{v}^{+}} c \Xi_{w}\left(Q_{v}\right), \quad v \in \operatorname{vert}(P) .
$$

СлеДСТвИЕ Е.20. Каждому блоку $B \in \mathcal{B}(P)$ coответствует некоторое слово $\boldsymbol{w}_{\text {в }}$ с коэффичиентом 1 и

$$
\Xi(P)=\sum \boldsymbol{w}_{B}, \quad B \in \mathcal{B}(P) .
$$


СлеДСтвие Е.21 (Р. Стенли [57]). Верна теорема Е.7, m.е. $\boldsymbol{c d}$-индекс $\Xi(P)$ любого многогранника $P$ равен сумме $\boldsymbol{c d}$-слов с неотрицательными коэфбиииентами.

Пользуясь тем, что $\boldsymbol{c d}$-индекс не зависит от выбора вектора $\boldsymbol{p}$ общего положения, запишем $\Xi(P)=(\Xi(P)+\Xi(P)) / 2$, где первое слагаемое получается при помощи $\boldsymbol{p}$, а второе - при помощи -p. Упрощая выражения для слагаемых, отвечающих вершинам, можно получить более простую формулу вида $\Xi(P)=\sum \Xi_{v}^{\prime}(P)$.

Tеорема Е.22. Пусть $P$ - выпуклый $n$-мерный многогранник. Тогда:

1) если $n=0$, то $P$ имеет одну вершину $v$ и $\Xi_{v}^{\prime}(P)=\Xi(P)=1$;

2) если $n>0$, то $\Xi_{v}^{\prime}(P)=\left[\boldsymbol{c} \Xi\left(Q_{v}\right)+\left(2 \boldsymbol{d}-\boldsymbol{c}^{2}\right) \Xi\left(R_{v}\right)\right] / 2, v \in \operatorname{vert}(P)$.

E.4. $\boldsymbol{c d}$-индекс и $h$-полином. Пусть $\operatorname{Sym}[\alpha, t]-$ градуированное кольцо симметрических полиномов от $\alpha$ и $t$, где $\operatorname{deg} \alpha=\operatorname{deg} t=2$. Опишем действие операторов $\boldsymbol{c}$ и $\boldsymbol{d}$ на этом кольце и получим известное соотношение, связывающее $\boldsymbol{c d}$-индекс и торический $h$-полином.

ОпрЕДЕЛЕНиЕ Е.23. Определим операторы

$$
\boldsymbol{c}, \boldsymbol{d}: \operatorname{Sym}[\alpha, t] \rightarrow \operatorname{Sym}[\alpha, t], \quad \operatorname{deg} \boldsymbol{c}=2, \quad \operatorname{deg} \boldsymbol{d}=4,
$$

задав их на коэффициентах полинома $\sum h_{i} \alpha^{i} t^{n-i}$ :

$$
\begin{aligned}
& \left(h_{0}, \ldots, h_{n}\right) \boldsymbol{c}= \begin{cases}\left(g_{0}, g_{1}, \ldots, g_{[n / 2]}, g_{[n / 2]}, \ldots, g_{1}, g_{0}\right), & \text { если } n \text { четно, } \\
\left(g_{0}, g_{1}, \ldots, g_{[n / 2]}, 0, g_{[n / 2]}, \ldots, g_{1}, g_{0}\right), & \text { если } n \text { нечетно, }\end{cases} \\
& \left(h_{0}, \ldots, h_{n}\right) \boldsymbol{d}= \begin{cases}\left(0, \ldots, 0, g_{[n / 2]}, 0, \ldots, 0\right), & \text { если } n \text { четно, } \\
(0, \ldots, 0), & \text { если } n \text { нечетно, }\end{cases}
\end{aligned}
$$

где $g_{0}=h_{0}$ и $g_{i}=h_{i}-h_{i-1}, i=1, \ldots,[n / 2]$.

УтВеРЖДЕНИЕ Е.24. Рассмотрим отображение $H_{\mathcal{R} \mathcal{P}}: \mathcal{R P} \rightarrow \operatorname{Sym}[\alpha, t]$ (см. пример 10.21). Имеем:

$$
H_{\mathcal{R} \mathcal{P}}(P \boldsymbol{c})=H_{\mathcal{R} \mathcal{P}}(P) \boldsymbol{c}, \quad H_{\mathcal{R P}}(P \boldsymbol{d})=H_{\mathcal{R} \mathcal{P}}(P) \boldsymbol{d} .
$$

Доказательство. Имеем: $P \boldsymbol{c}=\mathscr{A} P, P \boldsymbol{d}=\mathscr{D} P$. Тогда:

$$
\begin{aligned}
& \tau G_{\mathcal{R P}}(\mathscr{A} P)-G_{\mathcal{R P}}(\mathscr{A} P)=G_{\mathcal{R P}}(\Phi(\alpha-t) \mathscr{A} P-\mathscr{A} P) \\
& \quad=G_{\mathcal{R} \mathcal{P}}([(\alpha-t)+(\alpha-t) \Phi(\alpha-t)] P)=(\alpha-t)\left[G_{\mathcal{R P}}(P)+\tau G_{\mathcal{R P}}(P)\right],
\end{aligned}
$$

откуда

$$
H_{\mathcal{R P}}(\mathscr{A} P)=\frac{\tau G_{\mathcal{R P}}(\mathscr{A} P)-G_{\mathcal{R P}}(\mathscr{A} P)}{\alpha-t}=G_{\mathcal{R P}}(P)+\tau G_{\mathcal{R} \mathcal{P}}(P)=H_{\mathcal{R P}}(P) c .
$$

Теперь

$$
\begin{aligned}
& \tau G_{\mathcal{R P}}(\mathscr{D} P)-G_{\mathcal{R} \mathcal{P}}(\mathscr{D} P)=G_{\mathcal{R} \mathcal{P}}(\Phi(\alpha-t) \mathscr{D} P-\mathscr{D} P) \\
& \quad=G_{\mathcal{R P}}\left[(\alpha-t) \mathscr{A} P+(\alpha-t)^{2} P\right]=(\alpha-t)\left[G_{\mathcal{R} \mathcal{P}}(\mathscr{A} P)+(\alpha-t) G_{\mathcal{R P}}(P)\right] .
\end{aligned}
$$

Из предыдущих выкладок получаем формулу

$$
G_{\mathcal{R P}}(\mathscr{A} P)+(\alpha-t) G_{\mathcal{R P}}(P)=\tau G_{\mathcal{R P}}(\mathscr{A} P)-(\alpha-t) \tau G_{\mathcal{R P}}(P) .
$$


Пусть $P-n$-мерный многогранник. Тогда слева первое и второе слагаемые имеют по $\alpha$ степень не больше, чем $[(n+1) / 2]$ и $[n / 2]+1$ соответственно, а справа - не меньше, чем $n+2-[(n+1) / 2]$ и $n+1-[n / 2]$. Если $n=2 k$, то слева получаем числа $k$ и $k+1$, а справа $-k+2$ и $k+1$, поэтому в этом случае $G_{\mathcal{R P}}(\mathscr{A} P)+(\alpha-t) G_{\mathcal{R P}}(P)=g_{k}(P) \alpha^{k+1} t^{k+1}$. Если же $n=2 k+1$, то слева получаем числа $k+1$ и $k+1$, а справа $-k+2$ и $k+2$, поэтому $G_{\mathcal{R P}}(\mathscr{A} P)+(\alpha-t) G_{\mathcal{R} P}(P)=0$. Итак, в обоих случаях имеем:

$H_{\mathcal{R P}}(\mathscr{D} P)=\frac{\tau G_{\mathcal{R P}}(\mathscr{D} P)-G_{\mathcal{R P}}(\mathscr{D} P)}{\alpha-t}=G_{\mathcal{R} \mathcal{P}}(\mathscr{A} P)+(\alpha-t) G_{\mathcal{R} \mathcal{P}}(P)=H_{\mathcal{R} \mathcal{P}}(P) \boldsymbol{d}$.

Из доказательства мы получаем, что действие операторов $\boldsymbol{c}$ и $\boldsymbol{d}$ на симметрическом полиноме $h(\alpha, t)$ имеет вид (см. также [39]): $h \boldsymbol{c}=g(\alpha, t)+g(t, \alpha)$, $h \boldsymbol{d}=g \boldsymbol{c}+(\alpha-t) g$, где $g(\alpha, t)=\sum_{i=0}^{[n / 2]} g_{i} \alpha^{i} t^{n+1-i}$ и $g_{0}=h_{0}$, а $g_{i}=h_{i}-h_{i-1}$.

СлеДСтвие Е.25. Пусть $P \in \mathcal{P}$. Тогда $H_{\mathcal{R} \mathcal{P}}(P)=1 \Xi(P)$.

ДокАЗАТЕЛЬСтво. Из утверждения 10.22 имеем $H_{\mathcal{R P}}(P)=H_{\mathcal{R P}}\left(\pi_{\mathscr{A} \mathscr{D}}(P)\right)$, поэтому

$$
H_{\mathcal{R P}}(P)=H_{\mathcal{R} \mathcal{P}}\left(\pi_{\mathscr{A} \mathscr{D}}(P)\right)=H_{\mathcal{R P}}(1 \Xi(P))=1 \Xi(P) .
$$

Полином $H_{\mathcal{R}}(P)$ представляет собой однородную форму известного торического $h$-полинома, поэтому следствие Е.25 содержится в [45; теорема 5] и [39; лемма 7.9, предложение 7.10].

В качестве следствия мы получаем вычисление $h$-полинома при помощи движения гиперплоскости в следующем виде:

$$
h(\alpha, t)(P)=\sum h_{v}(\alpha, t)(P), \quad v \in \operatorname{vert}(P) .
$$

Этот результат следует из работы К. Ли [45], в которой он сформулирован для торического $h$-полинома.

Tеорема Е.26. Пусть $P$ - въпуклый $n$-мернъй многогранник. Тогда:

1) если $n=0$, то $P$ имеет одну вершину $v$ и $h_{v}(\alpha, t)(P)=h(\alpha, t)(P)=1$;

2) если $n>0$, то

$$
h_{v}(\alpha, t)(P)=h(\alpha, t)\left(R_{v}\right) \boldsymbol{d}+\sum_{w \in \operatorname{vert}\left(Q_{v}\right) \cap H_{v}^{+}} h_{w}(\alpha, t)\left(Q_{v}\right) \boldsymbol{c}, \quad v \in \operatorname{vert}(P) .
$$

ДокАЗАТЕЛЬство. Легко видеть, что $\boldsymbol{c d}$-индекс многогранника $P^{*}$ получается из $\boldsymbol{c d}$-индекса многогранника $P$ обращением порядка следования букв $\boldsymbol{c}$ и $\boldsymbol{d}$ в каждом мономе. Положим $\Xi\left(P^{*}\right)=\Xi^{*}(P)$. Тогда

$$
\Xi_{v}^{*}(P)=\Xi^{*}\left(R_{v}\right) \boldsymbol{d}+\sum_{w \in \operatorname{vert}\left(Q_{v}\right) \cap H_{v}^{+}} \Xi_{w}^{*}\left(Q_{v}\right) \boldsymbol{c}, \quad v \in \operatorname{vert}(P) .
$$

Тогда

$$
h(\alpha, t)(P)=H_{\mathcal{R} \mathcal{P}}\left(P^{*}\right)=1 \Xi\left(P^{*}\right)=1 \Xi^{*}(P),
$$

откуда непосредственно вытекает утверждение теоремы.

Из теоремы Е.22 следует еще одно рекуррентное представление $h$-полинома. 
Tеорема Е.27. Пусть $P$ - выпуклый $n$-мернъй многогранник. Тогда:

1) если $n=0$, то $P$ имеет одну вершину $v$ и $h_{v}^{\prime}(\alpha, t)(P)=h(\alpha, t)(P)=1$;

2) если $n>0$, mo

$$
h_{v}^{\prime}(\alpha, t)(P)=\frac{1}{2}\left[h(\alpha, t)\left(Q_{v}\right) \boldsymbol{c}+h(\alpha, t)\left(R_{v}\right)\left(2 \boldsymbol{d}-\boldsymbol{c}^{2}\right)\right], \quad v \in \operatorname{vert}(P) .
$$

E.5. Комментарии. К. Кару в [62] доказал гипотезу Р. Стенли [57] о том, что $\boldsymbol{c d}$-индекс любого горенштейнова* (см. [9]) частично упорядоченного множества неотрицателен. (В частности, из этого следует теорема Е.7.) Он использует еще одно представление $\boldsymbol{c d}$-индекса в следующем виде. Пусть $P$ $n$-мерный многогранник. Положим

$$
H_{n}(P)=\sum_{S \subseteq[0, n-1]} h_{S} t_{1}^{s_{1}} \cdots t_{n}^{s_{n}},
$$

где $s_{i}=1$, если $i-1 \in S$, и $s_{i}=0$ иначе. Каждому $\boldsymbol{c d}$-слову степени $2 n$ сопоставим полином от $t_{1}, \ldots, t_{n}$ по следующему правилу: начиная слева, заменим каждую букву $\boldsymbol{c}$ на $\left(t_{i}+1\right)$, а $\boldsymbol{d}-$ на $\left(t_{i}+t_{i+1}\right)$ в порядке возрастания индексов так, чтобы каждая переменная $t_{i}$ была использована ровно один раз.

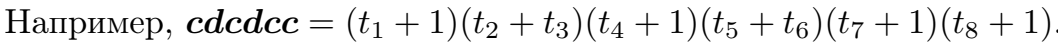

УтВеРЖДЕНИЕ Е.28. Имеем: $\Xi(P)=H_{n}(P)$.

Авторы благодарны А. А. Айзенбергу за ценные замечания и В. Д. Володину за метод, позволивший сократить доказательства в приложении А.

\section{Список литературы}

[1] V.M. Buchstaber, N. Ray, "An invitation to toric topology: Vertex four of a remarkable tetrahedron", Toric topology, Contemp. Math., 460, Amer. Math. Soc., Providence, RI, 2008, 1-27, ISBN: 978-0-8218-4486-1/pbk.

[2] В. М. Бухштабер, "Кольцо простых многогранников и дифференциальные уравнения", Геометрия, топология и математическая физика. I, Тр. МИАН, 263, МАИК, М., 2008, 18-42; англ. пер.: V. M. Buchstaber, "Ring of simple polytopes and differential equations", Proc. Steklov Inst. Math., 263:1 (2008), 13-37.

[3] В. М. Бухштабер, Н. Ю. Ероховец, "Алгебра операторов на кольце многогранников и квазисимметрические функции”, УМН, 65:2 (2010), 197-198; англ. пер.: V. M. Buchstaber, N. Yu. Erokhovets, "Algebra of operators on the ring of polytopes and quasi-symmetric functions", Russian Math. Surveys, 65:2 (2010), 381-383.

[4] V.M. Buchstaber, N. Erokhovets, Ring of polytopes, quasi-symmetric functions and Fibonacci numbers, arXiv: 1002.0810.

[5] V. M. Buchstaber, N. Erokhovets, Polytopes, Hopf algebras and quasi-symmetric functions, arXiv: 1011.1536v1.

[6] B. Grünbaum, Convex polytopes, Grad. Texts in Math., 221, Springer-Verlag, New York, 2003, ISBN: 0-387-00424-6; 0-387-40409-0, xvi+468 pp.

[7] G. M. Ziegler, Lectures on polytopes, Grad. Texts in Math., 152, Springer-Verlag, New York, 1995, ISBN: 0-387-94365-X, x+370 pp.

[8] А. Брондстед, Введение в теорию выпукльх многогранников, Мир, М., 1988, 240 с.; пер. с англ.: A. Brondsted, An introduction to convex polytopes, Grad. Texts in Math., 90, Springer-Verlag, New York-Heidelberg-Berlin, 1983, ISBN: 0-387-90722-X, viii+160 pp. 
[9] В. М. Бухштабер, Т. Е. Панов, Торические действия в топологии и комбинаторике, МЦНМО, М., 2004, ISBN: 5-94057-145-X, 272 c.

[10] S. A. Joni, G.-C. Rota, "Coalgebras and bialgebras in combinatorics", Stud. Appl. Math., 61:2 (1979), 93-139.

[11] W. R. Schmitt, "Antipodes and incidence coalgebras", J. Combin. Theory Ser. A, 46:1-2 (1987), 264-290.

[12] W.R. Schmitt, "Incidence Hopf algebras", J. Pure Appl. Algebra, 96:3 (1994), 299-330.

[13] R. Ehrenborg, "On posets and Hopf algebras", Adv. Math., 119:1 (1996), 1-25.

[14] M. Aguiar, N. Bergeron, F. Sottile, "Combinatorial Hopf algebras and generalized Dehn-Sommerville relations", Compos. Math., 142:1 (2006), 1-30; arXiv: math/0310016.

[15] N. Ray, W. Schmitt, "Combinatorial models for coalgebraic structures", Adv. Math., 138:2 (1998), 211-262.

[16] H. S. M. Coxeter, Regular polytopes, 3rd edition, Dover Publications, New York, 1973.

[17] M. M. Bayer, L. J. Billera, "Generalized Dehn-Sommerville relations for polytopes, spheres and Eulerian partially ordered sets", Invent. Math., 79:1 (1985), 143-157.

[18] J. Milnor, J. Moore, "On the structure of Hopf algebras", Ann. of Math. (2), 81:2 (1965), 211-264.

[19] К. Кассель, Квантовые группь, ФАЗИС, М., 1999, 688 с.; пер. с англ.: Ch. Kassel, Quantum groups, Grad. Texts in Math., 155, Springer-Verlag, New York, 1995.

[20] M. Hazewinkel, "The algebra of quasi-symmetric functions is free over the integers", Adv. Math., 164:2 (2001), 283-300.

[21] A. Baker, B. Richter, "Quasisymmetric functions from a topological point of view", Math. Scand., 103:2 (2008), 208-242.

[22] K. T. Chen, R.H. Fox, R. C. Lyndon, "Free differential calculus. IV. The quotient groups of the lower central series", Ann. of Math. (2), 68:1 (1958), 81-95.

[23] R. P. Stanley, Ordered structures and partitions, Mem. Amer. Math. Soc., 119, Amer. Math. Soc., Providence, RI, 1972, iii+104 pp.

[24] I. M. Gessel, "Multipartite P-partitions and inner product of skew Schur functions", Combinatorics and algebra (Boulder, CO, 1983), Contemp. Math., 34, Amer. Math. Soc., Providence, RI, 1984, 289-317.

[25] I. M. Gelfand, D. Krob, A. Lascoux, B. Leclerc, V.S. Retakh, J.-Y. Thibon, "Noncommutative symmetric functions", Adv. Math., 112:2 (1995), 218-348.

[26] C. Malvenuto, C. Reutenauer, "Duality between quasi-symmetric functions and the Solomon descent algebra", J. Algebra, 177:3 (1995), 967-982.

[27] М. Хазевинкель, “Обобщенные перекрывающие тасовочные алгебры”, Труды международной конферениии, посвященной 90-летию со дня рождения Л. С. Понтрягина: Алгебра (Москва, 31 августа - 6 сентября 1998 г.), Итоги науки и техники. Соврем. матем. и ее прил. Темат. обз., 69, №8, ВИНИТИ, М., 1999, 193-222; англ. изд.: M. Hazewinkel, "Generalized overlapping shuffle algebras", J. Math. Sci., 106:4 (2001), 3168-3186.

[28] J. R. Stembridge, "Enriched p-partitions", Trans. Amer. Math. Soc., 349:2 (1997), 763-788.

[29] J.-Y. Thibon, B.-C.-V. Ung, "Quantum quasi-symmetric functions and Hecke algebras", J. Phys. A, 29:22 (1996), 7337-7348.

[30] Б. И. Ботвинник, В. М. Бухштабер, С. П. Новиков, С. А. Юзвинский, "Алгебраические аспекты теории умножений в комплексных кобордизмах", УМН, 55:4 (2000), 5-24; англ. пер.: В. I. Botvinnik, V. M. Buchstaber, S. P. Novikov, S. A. Yuzvinsky, "Algebraic aspects of the theory of multiplications in complex cobordism theory", Russian Math. Surveys, 55:4 (2000), 613-633. 
[31] L. J. Billera, N. Liu, "Non-commutative enumeration in graded posets", J. Algebraic Combin., 12:1 (2000), 7-24.

[32] С. П. Новиков, "Различные удвоения алгебр Хопфа. Алгебры операторов на квантовых группах, комплексные кобордизмы”, УМH, 47:5 (1992), 189-190; англ. пер.: S.P. Novikov, "Various doublings of Hopf algebras. Operator algebras on quantum groups, complex cobordisms", Russian Math. Surveys, 47:5 (1992), 198-199.

[33] C. Reutenauer, Free Lie algebras, London Math. Soc. Monogr. (N.S.), 4, The Clarendon Press, Oxford Univ. Press, New York, 1993, ISBN: 0-19-853679-8, xviii+269 pp.

[34] Combinatorics on words, Encyclopedia Math. Appl., 17, ed. M. Lothaire, Addison-Wesley, Reading, MA, 1983, ISBN: 0-201-13516-7, xix+238 pp.

[35] R. Bott, H. Samelson, "On the Pontryagin product in spaces of paths", Comment. Math. Helv., 27 (1953), 320-337.

[36] V. M. Buchstaber, J. Grbic, Lie-Hopf algebras and loop homology of suspension spaces, arXiv: 1011.2549 .

[37] R. P. Stanley, "Generalized $h$-vectors, intersection cohomology of toric varieties, and related results", Commutative algebra and combinatorics (Kyoto, 1985), Adv. Stud. Pure Math., 11, North-Holland, Amsterdam, 1987, 187-213.

[38] L. J. Billera, S. K. Hsiao, S. van Willigenburg, "Peak quasisymmetric functions and Eulerian enumeration", Adv. Math., 176:2 (2003), 248-276; 24 June 2007, arXiv: $0706.3486 \mathrm{v} 1$.

[39] M. M. Bayer, R. Ehrenborg, "The toric $h$-vector of partially ordered sets", Trans. Amer. Math. Soc., 352:10 (2000), 4515-4531.

[40] G. Kalai, "A new basis for polytopes", J. Combin. Theory Ser. A, 49:2 (1988), 191-209.

[41] M. M. Bayer, A. Klapper, "A new index for polytopes", Discrete Comput. Geom., 6:1 (1991), 33-47.

[42] M. M. Bayer, C. W. Lee, "Combinatorial aspects of convex polytopes", Handbook of convex geometry, A, North-Holland, Amsterdam, 1993, 485-534.

[43] J. Fine, A complete h-vector for convex polytopes, arXiv: 0911.5722.

[44] J. Fine, A complete g-vector for convex polytopes, arXiv: 1001.1562.

[45] C.W. Lee, Sweeping the cd-index and the toric h-vector, 2009, www.ms.uky.edu/ lee/cd.pdf; arXiv: 1011.2264.

[46] C. Stenson, "Relationships among flag $f$-vector inequalities for polytopes", Discrete Comput. Geom., 31:2 (2004), 257-273.

[47] P. McMullen, "The numbers of faces of simplicial polytopes", Israel J. Math., 9:4 (1971), 559-570.

[48] R. P. Stanley, "The number of faces of simplicial convex polytope", Adv. in Math., 35:3 (1980), 236-238.

[49] L. J. Billera, C. W. Lee, "A proof of sufficiency of McMullen's conditions for $f$-vectors of simplicial polytopes", J. Combin. Theory Ser. A, 31:3 (1981), 237-255.

[50] P. McMullen, "On simple polytopes", Invent. Math., 113:2 (1993), 419-444.

[51] K.-H. Fieseler, "Rational intersection cohomology of projective toric varieties", J. Reine Angew. Math., 413 (1991), 88-98.

[52] K. Karu, "Hard Lefschetz theorem for nonrational polytopes", Invent. Math., 157:2 (2004), 419-447; arXiv: math/0112087.

[53] G. M. Ziegler, "Face numbers of 4-polytopes and 3-spheres", Proceedings of the International Congress of Mathematicians (Beijing, China, 2002), III, Higher Ed. Press, Beijing, 2002, 625-634; arXiv: math/0208073.

[54] L. J. Billera, G. Hetyei, "Linear inequalities for flags in graded partially ordered sets", J. Combin. Theory Ser. A, 89:1 (2000), 77-104; arXiv: math/9706220. 
[55] N. Bergeron, S. Mykytiuk, F. Sottile, S. van Willigenburg, "Shifted quasi-symmetric functions and the Hopf algebra of peak functions", Discrete Math., 246:1-3 (2002), $57-66$.

[56] N. Bergeron, S. Mykytiuk, F. Sottile, S. van Willigenburg, "Noncommutative Pieri operators on posets", J. Combin. Theory Ser. A, 91:1-2 (2000), 84-110; arXiv: math/0002073.

[57] R. P. Stanley, "Flag $f$-vectors and the $c d$-index", Math. Z., 216:1 (1994), 483-499.

[58] L. J. Billera, R. Ehrenborg, "Monotonicity of the $c d$-index for polytopes", Math. Z., 233 (2000), 421-441.

[59] L. J. Billera, R. Ehrenborg, M. Readdy, "The $c d$-index of zonotopes and arrangements", Mathematical essays in honor of Gian-Carlo Rota (Cambridge, MA, 1996), Progr. Math., 161, Birkhäuser, Boston, 1998, 23-40.

[60] R. Ehrenborg, H. Fox, "Inequalities for $c d$-indices of joins and products of polytopes", Combinatorica, 23:3 (2003), 427-452.

[61] R. Ehrenborg, M. Readdy, "Coproducts and the $c d$-index", J. Algebraic Combin., 8:3 (1998), 273-299.

[62] K. Karu, "The $c d$-index of fans and posets", Compos. Math., 142:3 (2006), 701-718.

B. M. Бухштабер (V.M. Buchstaber)

Поступила в редакцию

Математический институт им. В. А. Стеклова РАН

01.03 .2011

E-mail: buchstab@mi.ras.ru

\section{Н. Ю. Ероховец (N. Yu. Erokhovets)}

Московский государственный университет

им. М. В. Ломоносова

E-mail: erochovetsn@hotmail.com 